\title{
Scalable Domain Decomposition Algorithms for Uncertainty Quantification in High Performance Computing
}

by

\author{
Ajit Desai
}

A thesis submitted to the Faculty of Graduate and Postdoctoral Affairs in partial fulfillment of the requirements for the degree of

\author{
Doctor of Philosophy \\ in \\ Civil Engineering \\ Carleton University \\ Ottawa, Ontario
}

(C)2018

Ajit Desai 


\begin{abstract}
Uncertainty quantification of practical engineering applications using the intrusive spectral stochastic finite element methods (SSFEM) may involve solving a system of linear equations in the order of billions of unknowns. Therefore, in this thesis the intrusive polynomial chaos expansion (PCE) based two-level domain decomposition (DD) algorithms for stochastic partial differential equations (PDEs) are extended to handle high resolution numerical models using an in-house scalable parallel solvers toolkit. First, attention is given to facilitate the numerical simulation of the elliptic stochastic PDEs with a large number of random variables to address the so-called curse of dimensionality issue. Second, for three-dimensional coupled stochastic PDE systems such as equations of linear elasticity, the extended wirebasket-based coarse grid is developed to improve the performance and overcome the scalability issues of the DD based iterative solvers with a vertex-based coarse grid. Third, the developed DD solvers for the SSFEM are coupled with FEniCS deterministic finite element assembly routines in order to reduce the coding required for the implementation and generalize the application of these solvers to a variety of PDEs using FEniCS. Fourth, the intrusive SSFEM with scalable DD solver is shown to outperform the non-intrusive SSFEM with the sparse grid quadrature for a stochastic PDE with the non-Gaussian random variables. This highlights the advantages of the intrusive approach and demonstrates the necessity of scalable parallel solvers for uncertainty quantification. This thesis also elaborates on the HPC implementational aspects of the DD solvers for SSFEM. Three-level nested sparse iterative solvers, which employ an efficient DD based preconditioners are used to simulate two and three-dimensional scalar and vector-valued stochastic PDEs. The random system parameters and the solution process are modeled as a non-Gaussian
\end{abstract}


stochastic process characterized by using up to 25 random variables with a third-order expansion resulting in 3276 PCE coefficients. Numerical and parallel scalabilities of these algorithms are investigated employing large-scale high-performance computing clusters with MPI, PETSc and FEniCS libraries. For all these scalability measurements, we report the results for different numbers of random variables and different orders of PCE to measure the sensitivity of the algorithms to the stochastic parameters. 
Dedicated to my wife, Pragati, and my son, Prajit. 


\section{Acknowledgments}

I am greatly indebted to my research supervisors, Prof. Abhijit Sarkar, Prof. Chris Pettit and Prof. Dominique Poirel for all their academic supervision and support they provided throughout the duration of this research. I want to thank Dr. Mohammad Kahlil for his guidance from the start of my Ph.D. I could not have succeeded in finishing this work without their consistent assistance, advice and encouragement.

I also extend my sincere thanks to my thesis committee: Prof. Steven Wojtkiewicz, Prof. Emmanuel Lorin, Prof. Majid Mohammadian and Prof. Heng Khoo, for their insightful comments and encouragement.

Many more people have helped me, directly or indirectly, knowingly or unknowingly, at different stages. I want to thank Rimple Sandhu, Phillipee Bissalion, Pranjal Prasad and the anonymous reviewers of my papers. I also want to thank my friends, Sandip and Revanth for continuously encouraging me throughout my Ph.D.

Over the last four years, I was supported by the Ontario Trillium Scholarship (OTS) for which I am grateful. I also want to thank Carleton University for the internal scholarships I was provided with.

This thesis would not have been possible unless the support and love of my parents. Thanks to all the sacrifice that they made throughout their life for seeing me reach this day. I owe my deepest gratitude to my elder brother, Amol for his support and guidance throughout my career.

Last but not least, I want to thank my wife, Pragati who always helped me to surmount all obstacles over these last few years. I am indebted to her for the endless love, emotional support and encouragement along the way. 


\section{Contents}

1 Introduction 1

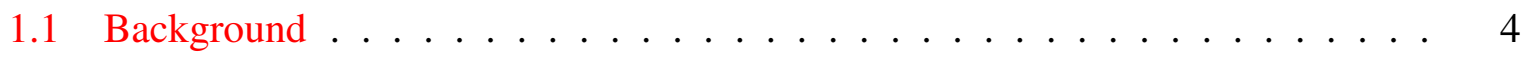

1.2 Brief Overview of Spectral Stochastic Finite Element

Methods for Uncertainty Quantification $\ldots \ldots \ldots$. . . . . . . 7

1.3 Brief Overview of Domain Decomposition Methods

for Uncertainty Quantification . . . . . . . . . . . . . . . . 10

1.4 Brief Overview of High-Performance Computing

in Uncertainty Quantification . . . . . . . . . . . . . . . . . . 14

1.5 Literature Review of Solution Methods for Intrusive

Spectral Stochastic Finite Element Methods . . . . . . . . . . . . . 16

1.6 Problem Statement . . . . . . . . . . . . . . . . . . . . . 22

1.7 Research Objectives . . . . . . . . . . . . . . . . . . . . . . 25

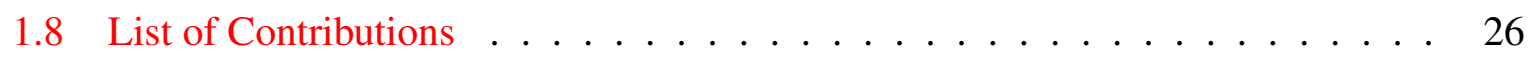

1.9 Thesis Organization $\ldots \ldots \ldots \ldots \ldots \ldots \ldots \ldots \ldots \ldots \ldots \ldots \ldots$

2 Spectral Stochastic Finite Element Methods for Stochastic PDEs: Intrusive Versus Non-Intrusive Approach

2.1 Spectral Representation of Stochastic Processes . . . . . . . . . . . . . 33

2.1.1 Karhunen-Loève Expansion . . . . . . . . . . . . . . . . . . . 34

2.1.2 Polynomial Chaos Expansion $\ldots \ldots \ldots \ldots . \ldots . \ldots 37$ 
2.2 Spectral Stochastic Finite Element Methods . . . . . . . . . . . . . . . . 39

2.2 .1 Intrusive SSFEM $\ldots \ldots \ldots \ldots \ldots \ldots \ldots$

2.2.2 Non-Intrusive SSFEM Approach . . . . . . . . . . . . . . . . . . 49

2.3 Comparison of Intrusive and Non-Intrusive SSFEM . . . . . . . . . . 53

3 Domain Decomposition of Stochastic PDEs in Two Dimensions: Adaptations to Large Random Variables $\quad 64$

3.1 Domain Decomposition of Stochastic PDEs . . . . . . . . . . . . 67

3.1.1 Non-Overlapping Domain Decomposition - the Primal Approach . . . 71

3.1.2 Non-Overlapping Domain Decomposition - the Dual-Primal Approach 80

3.2 Parallel Implementation of Stochastic Domain Decomposition Solvers . . . . . 85

3.2.1 Parallel PCGM Algorithm . . . . . . . . . . . . . . . 85

3.2.2 Implementation of BDDC/NNC-PCGM Algorithm Using PETSc $\ldots . .86$

3.2.3 Implementation of FETI-DP Algorithm using PETSc . . . . . . . . . 94

3.3 Numerical Experiments . . . . . . . . . . . . . . . . . . . . . . . . . . 97

3.3.1 Experimental Framework . . . . . . . . . . . . . . . . 98

3.3.2 Characteristics of the Solution Process . . . . . . . . . . . . . . . 99

3.3.3 Scalabilities Studies for Stochastic Simulations _ . . . . . . . . . 100

3.3.4 Scalability Results for BDDC/NNC-PCGM Solver . . . . . . . . . 103

3.3.5 Scalability Results for FETI-DP-PCGM Solver . . . . . . . . . . . 113

3.3.6 Comparison with Non-Intrusive SSFEM at High Dimensions . . . . . 115

4 Domain Decomposition of Stochastic PDEs in Three Dimensions: Development of $\begin{array}{ll}\text { Probabilistic Wirebasket Coarse Grid } & 118\end{array}$

4.1 Extended Schur Complement of Coupled Stochastic PDE System . . . . . . . 125

4.2 Two-Level Preconditioner using Extended Wirebasket-Based Coarse Grid . . . 131

4.3 Three-Dimensional Poisson Problem . . . . . . . . . . . . . . . . 135

4.3.1 Numerical Experimental Framework . . . . . . . . . . . . . . 136 
4.3.2 Characteristics of the Solution Process . . . . . . . . . . . . . 137

4.3.3 Comparison of Extended Wirebasket with Vertex Coarse Grid . . . . 142

4.3.4 Scalabilities Studies for Stochastic Simulations . . . . . . . . . . 145

4.4 Three-Dimensional Linear Elasticity Problem . . . . . . . . . . . . . . 152

4.4.1 Numerical Experimental Framework . . . . . . . . . . . . . . . 155

4.4.2 Characteristics of the Solution Process . . . . . . . . . . . 156

4.4.3 Comparison of Extended Wirebasket with Vertex Coarse Grid . . . . . 164

4.4.4 Scalabilities Studies for Stochastic Simulations . . . . . . . . . . 167

5 Summary of Research Findings and Future Directions 174

5.1 Summary of Research Findings . . . . . . . . . . . . . . . . . . . . 174

5.2 Limitations of the Study . . . . . . . . . . . . . . . . . 178

5.3 Future Research Directions . . . . . . . . . . . . . . . . . . . 180

5.3.1 Direct Extensions of the Study . . . . . . . . . . . . . . 180

5.3.2 Broader Issues to be Covered in Future Work . . . . . . . . . . . 181

5.4 Conclusions . . . . . . . . . . . . . . . . . . . 183

$\begin{array}{ll}\text { Appendices } & 185\end{array}$

A Spectral Representation of Stochastic Process 185

A.1 Karhunen-Loève Expansion ～. . . . . . . . . . . . . . . . . . . 185

A.2 Polynomial Chaos Expansion . . . . . . . . . . . . . . . . . . . 189

A.3 Spectral Representation of Lognormal Stochastic

Process using PCE . . . . . . . . . . . . . . 193

B Spectral Stochastic Finite Element Method Formulation, Algorithms and Codes 196

B.1 Spectral Stochastic Finite Element Methods . . . . . . . . . . . . . . . 197

B.2 Intrusive SSFEM . . . . . . . . . . . . . . . . . 198

B.3 Non-Intrusive SSFEM . . . . . . . . . . . . . . . 206 
C Numerical Validation of Parallel Domain Decomposition Code

D Mesh Decomposition in Two and Three Dimensions 


\section{List of Figures}

1.1 Uncertainty propagation framework for large-scale stochastic PDEs. . . . . . . 2

1.2 Implementational framework of DD-based SSFEM package for large-scale discretized stochastic PDEs in high-performance computing. . . . . . . . . . . 3

2.1 Relative partial sum of eigenvalues for exponential covariance kernel on a unit

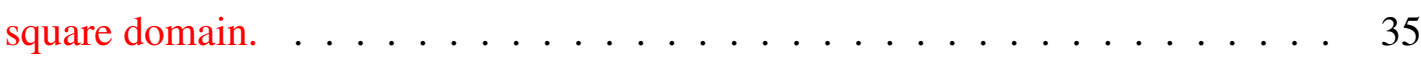

2.2 Finite element mesh and the solution field at the mean value of stochastic system

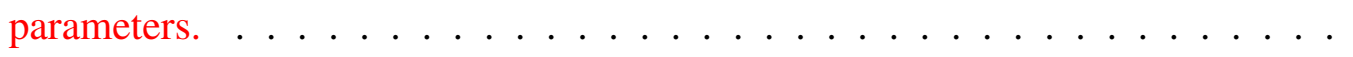

2.3 SSFEM system matrices for the fixed $N=30, p_{\mathrm{A}}=2, p_{u}=3$ with $L=5$, $L=10$ and $L=15 \ldots \ldots \ldots \ldots \ldots \ldots$

2.4 SSFEM system matrices for the fixed $N=30, p_{\mathrm{A}}=2, L=3$ with $p_{u}=5$, $p_{u}=10$ and $p_{u}=15 \ldots \ldots \ldots \ldots \ldots$

2.5 Number of quadrature points for a fixed $d=2$ with $l=3$ and $l=6$. . . . . . 52

2.6 Block-sparse structures of the intrusive system matrices for a fixed mesh resolution with $L=3$ and $p_{u}=4,5 \ldots \ldots \ldots \ldots$

2.7 Block-sparse structures of the intrusive system matrices for a fixed mesh resolution with $p_{u}=3$ and $L=4,5 \ldots \ldots \ldots \ldots$

2.8 Error norms in $0^{t h}, 1^{\text {st }}$ and $2^{\text {nd }}$ order PCE coefficients for $L=2 \ldots \ldots$

2.9 Error norms in $0^{t h}, 1^{\text {st }}$ and $2^{\text {nd }}$ order PCE coefficients for $L=3 . \ldots$. . . . 58

2.10 Error norms in $0^{t h}, 1^{\text {st }}$ and $2^{\text {nd }}$ order PCE coefficients for $L=4 \ldots \ldots$ 
2.11 Comparison of relative error norms between intrusive and non-intrusive SSFEM in $0^{t h}, 1^{\text {st }}$ and $2^{\text {nd }}$ order PCE coefficients for $L=5 \ldots \ldots \ldots \ldots$

2.12 Comparison of relative error norms between intrusive and non-intrusive SSFEM in the standard deviation (computed for all nodes) of the solution process for $L=2,3,4$ and $5 \ldots \ldots \ldots \ldots \ldots \ldots$

2.13 Comparison of error surface plots between intrusive and non-intrusive SSFEM for $L=4$ in the standard deviation of the solution process using $p_{u}=3,4,5$ and $l=3,4,5$ with respect to standard deviation of the solution process using $p_{u}=6$ and $l=6 \ldots \ldots \ldots \ldots \ldots$

3.1 One-level mesh decomposition showing • interior $(I)$ and -interface $(\Gamma)$ nodes. 71

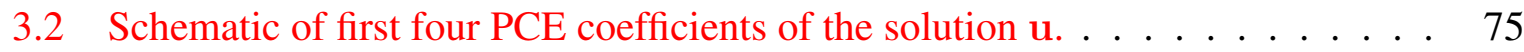

3.3 First four PCE coefficients of the solution with two-level mesh decomposition showing interior(i), aremaining(r) and $\star$ corner(c) nodes. . . . . . . . . . . 77

3.4 First four PCE coefficients of the solution showing FETI-DP mesh decomposition with .interior(i), remaining(r) and $\star$ corner(c) nodes. . . . . . . . . . . . 84

3.5 Flowchart for BDDC/NNC-PCGM solver. . . . . . . . . . . . . . . . 94

3.6 Flowchart for FETI-DP PCGM solver. . . . . . . . . . . . . . . . . . . . . 97

3.7 Typical finite element mesh with 1680 subdomains. . . . . . . . . . . . . . . 98

3.8 Mean and standard deviation of the solution process $u \ldots \ldots$. . . . . . . 100

3.9 Selected PCE coefficients of the solution process $u \ldots \ldots$. . . . . . . . 101

3.10 Relative partial sum of eigenvalues with respect to eigenvalue-index. . . . . . . 102

3.11 Need of DDM based solvers with the aid of computer cluster for stochastic simulations. . . . . . . . . . . . . . . . 103

3.12 Iteration count versus number of subdomains for the fixed mesh resolution with fixed number of PCE terms. . . . . . . . . . . . . . . . . . . . 104

3.13 Iteration count versus number of subdomains for the fixed problem size per core with increasing mesh resolution (fixed number of PCE terms). . . . . . . . . 105 
3.14 Execution time versus number of subdomains with the fixed mesh resolution versus the number of PCE terms. . . . . . . . . . . . . . . . . . 106

3.15 Execution time versus number of subdomains for the fixed problem size per core with increasing mesh resolution (fixed number of PCE terms). . . . . . . 106

3.16 Iteration count versus number PCE terms for the fixed mesh resolution with fixed number of subdomains. . . . . . . . . . . . . . . . . . . . . . 107

3.17 Iteration count versus number of subdomains for the fixed problem size per core with increasing number of PCE terms (fixed mesh resolution). . . . . . . . 108

3.18 Execution time versus number of subdomains for fixed problem size per subdomain with increasing number of PCEs (fixed mesh resolution). . . . . . . . . 108

3.19 Iteration count versus order of expansion for the fixed mesh resolution with fixed number of subdomains (fixed number of RVs). . . . . . . . . . . . 110

3.20 Iteration count versus number of subdomains for the fixed mesh resolution with fixed number of PCE terms. . . . . . . . . . . . . . . . . . . . . . . . 111

3.21 Iteration count versus number PCE terms for the fixed mesh resolution with fixed number of subdomains. . . . . . . . . . . . . . . . . . . . . 111

3.22 Execution time versus number of subdomains with the fixed mesh resolution and the fixed number of PCEs. . . . . . . . . . . . . . . . . . 112

3.23 Iteration count versus number PCEs for the fixed mesh resolution with fixed number of subdomains. . . . . . . . . . . . . . . . . . . . . . . . . . . 114

3.24 Iteration count versus number of subdomains for the fixed mesh resolution with fixed number of PCEs. . . . . . . . . . . . . . . . . . . . . . . . . . . . . 114

3.25 Iteration count versus number of subdomains for the fixed mesh resolution with fixed number of PCEs. . . . . . . . . . . . . . . . . . . . . . 115

3.26 Comparison of execution time for intrusive and non-intrusive SSFEM with the fixed mesh resolution and $L=5,10,15,20 \ldots \ldots \ldots \ldots \ldots$ 
4.1 Schematic representation of a simple wirebasket coarse grid for a cube partitioned into two subdomains, showing (-) for the global interface edge, (•) as vertices, $(\star)$ as interface-edges and $(\bullet)$ as interface-faces. . . . . . . . . . .

4.2 Schematic representation of wirebasket coarse grid for a cube partitioned into (a) four subdomains and (b) eight subdomains, showing (-) for the global interface edge, $(\bullet)$ as vertices and $(\star)$ as interface-edges. . . . . . . . . . . . 121

4.3 Subdomain-level system matrix $\mathbf{A}^{s}(\theta)$ computed using the mean system parameters, indicating coupling among $x, y$ and $z$ displacements. Also displays decomposed components for $x$ displacements according to interior $I$ and interface $\Gamma$ nodes. . . . . . . . . . . . . . . . . . . .

4.4 Subdomain-level stiffness matrix $\boldsymbol{A}_{I I}^{s}(\theta)$ for interior nodes, computed using the mean system parameters, indicating coupling among $x, y$ and $z$ displacements. 128

4.5 A typical three-dimensional finite element mesh partitioned into 320 subdomains. 136

4.6 Relative partial sum of eigenvalues with respect to eigenvalue-index. . . . . . . 137

4.7 Mean and standard deviation of the solution process $\mathscr{U} \ldots \ldots$. . . . . . . 138

4.8 Selected PCE coefficients of the solution process $\mathcal{U} \ldots \ldots$. . . . . . . . . 139

4.9 Selected PCE coefficients of the solution process $\mathcal{U} \ldots \ldots$. . . . . . . . . . 140

4.10 Selected PCE coefficients of the solution process $\mathscr{U} \ldots \ldots$. . . . . . . . . . . 141

4.11 Iteration count versus number of subdomains for the fixed mesh resolution with fixed number of PCE terms. . . . . . . . . . . . . . . . . . 142

4.12 Iteration count versus number of subdomains for fixed problem size per subdomain with increasing number of PCEs (fixed mesh resolution). . . . . . . . . . 143

4.13 Execution time versus number of subdomains for fixed problem size per subdomain with increasing number of PCEs (fixed mesh resolution). . . . . . . . . . 144

4.14 Iteration count versus number PCE terms for the fixed mesh resolution with fixed number of subdomains. . . . . . . . . . . . . . . . . . . 145 
4.15 Iteration count versus number of subdomains for the fixed mesh resolution with fixed number of PCE terms. . . . . . . . . . . . . . . . . 146

4.16 Iteration count versus number of subdomains for the fixed problem size per core with increasing mesh resolution (fixed number of PCE terms). . . . . . . . . . 147

4.17 Execution time versus number of subdomains with the fixed mesh resolution and the number of PCE terms. . . . . . . . . . . . . . . . . . . 148

4.18 Execution time versus number of subdomains for the fixed problem size per core with increasing mesh resolution (fixed number of PCE terms). . . . . . . . 148

4.19 Iteration count versus number PCE terms for the fixed mesh resolution with fixed number of subdomains. . . . . . . . . . . . . . . . .

4.20 Iteration count versus number of subdomains for the fixed problem size per core with increasing number of PCE terms (fixed mesh resolution). . . . . . . . . . 150

4.21 Execution time versus number of subdomains for fixed problem size per subdomain with increasing number of PCEs (fixed mesh resolution). . . . . . . . . . 15(

4.22 Iteration count versus order of expansion for the fixed mesh resolution with fixed number of subdomains (fixed number of RVs). . . . . . . . . . . .

4.23 A typical three-dimensional finite element mesh for a clamped beam partitioned into 320 subdomains. . . . . . . . . . . . . . . . . . . 156

4.24 Mean magnitude of the beam deflection subjected to self-weight. . . . . . . . 157

4.25 Magnitude and $x, y$ and $z$ components of the mean and standard deviation of the solution process $\mathcal{U} \ldots \ldots \ldots \ldots \ldots$

4.26 Magnitude and $x, y$ and $z$ components of the selected PCE coefficients of the solution process $\mathcal{U} \ldots \ldots \ldots \ldots$. . . . . . . . . . . . 160

4.27 Magnitude and $x, y$ and $z$ components of the selected PCE coefficients of the solution $\operatorname{process} \mathcal{U} \ldots \ldots \ldots \ldots \ldots$. . . . . . . . . . . . . . . . . . .

4.28 Magnitude and $x, y$ and $z$ components of the selected PCE coefficients of the solution process $\mathcal{U} \ldots \ldots \ldots \ldots \ldots \ldots$ 
4.29 Magnitude and $x, y$ and $z$ components of the selected PCE coefficients of the

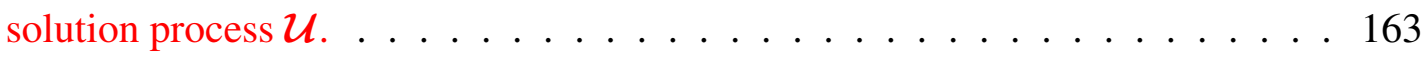

4.30 Iteration count versus number of subdomains for the fixed mesh resolution with fixed number of PCE terms. . . . . . . . . . . . . . . . . . . . . . . . . . 164

4.31 Iteration count versus number of subdomains for fixed problem size per subdomain with increasing number of PCEs (fixed mesh resolution). . . . . . . . 165

4.32 Execution time versus number of subdomains for fixed problem size per subdomain with increasing number of PCEs (fixed mesh resolution). . . . . . . . 166

4.33 Iteration count versus number of PCE terms for the fixed mesh resolution with fixed number of subdomains. . . . . . . . . . . . . . . . . . 166

4.34 Iteration count versus number of subdomains for the fixed mesh resolution with fixed number of PCE terms. . . . . . . . . . . . . . . . . . . . . . 168

4.35 Iteration count versus number of subdomains for the fixed problem size per core with increasing mesh resolution (fixed number of PCE terms). . . . . . . 168

4.36 Execution time versus number of subdomains with the fixed mesh resolution and the number of PCE terms. . . . . . . . . . . . . . . . . . . . . 169

4.37 Execution time versus number of subdomains for the fixed problem size per core with increasing mesh resolution (fixed number of PCE terms). . . . . . . . 170

4.38 Iteration count versus number of PCE terms for the fixed mesh resolution with fixed number of subdomains. . . . . . . . . . . . . . . . . . 171

4.39 Iteration count versus number of subdomains for the fixed problem size per core with increasing number of PCE terms (fixed mesh resolution). . . . . . . . . 171

4.40 Iteration count versus order of expansion for the fixed mesh resolution with fixed number of subdomains (fixed number of RVs) . . . . . . . . . . . . 172

A.1 Eigenvalues for $b=1.0$ and $a=0.5 \ldots \ldots \ldots \ldots \ldots \ldots$

A.2 Relative partial sum of eigenvalues for $b=1.0$ and $a=0.5 \ldots \ldots \ldots 8$ 
B.1 Mean and standard deviation of the solution field. . . . . . . . . . . . . 205

B.2 Selected PCE coefficients of the solution field. . . . . . . . . . . . . . 205

B.3 Two-dimensional sparse grid with $l=3$ and $l=4 . \ldots . . . . . . . .208$

B.4 Three-dimensional sparse grid with $l=3$ and $l=4 \ldots$. . . . . . . . . . 208

B.5 Mean and standard deviation of the solution field. . . . . . . . . . . . . . 210

B.6 Selected PCE coefficients of the solution field. . . . . . . . . . . . . . . 210

C.1 Validation of parallel NNC/BDDC-PCGM solver against MCS: the probability density function of the solution process at two nodal points. . . . . . . . . . . . 212

C.2 Validation of parallel NNC/BDDC-PCGM solver against MCS: the mean and standard deviation of the solution process (over the entire domain) using parallel solvers and MCS, respectively. . . . . . . . . . . . . . . 213

D.1 Implementational framework for mesh partitioning for non-overlapping domain decomposition methods. . . . . . . . . . . . . . 215

D.2 A typical geometry and mesh for square domain partitioned into four subdomains highlighting the nodes and elements. . . . . . . . . . . . . 217

D.3 A typical mesh for square domain partitioned into four subdomains highlighting corner nodes as $(\star) \ldots \ldots \ldots \ldots \ldots \ldots \ldots$. . . . . . . . . . . . . . . . . . . . . . .

D.4 A typical mesh for rectangular beam partitioned into four subdomains. . . . . . 218

D.5 A typical mesh data files extracted from the mesh file. . . . . . . . . . . 232 


\section{List of Tables}

1.1 Intrusive SSFEM system matrix size with the number of RVs. . . . . . . . . . 23

1.2 Number of quadrature points with the level of quadrature. . . . . . . . . . . . 23

2.1 Number of sample (quadrature) points $N_{s}$ in the sparse grid with level $l$ and the number of random variables $L \ldots \ldots$. . . . . . . . . . . . . 60

3.1 Floating-point operations for different number of cores with the fixed number

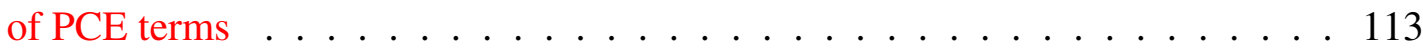

3.2 Floating-point operations for different number of PCE terms with the fixed number of cores . . . . . . . . . . . . . . . . . . 113

A.1 Tensor product of one-dimensional eigenvalues. . . . . . . . . . . . . . 187

A.2 One-dimensional omegas $\omega_{i}$ and eigenvalues $\lambda_{i}^{x}, i=1,2, \ldots, 7 \ldots \ldots 187$

A.3 Two-dimensional eigenvalues $\lambda_{n}, n=1,2, \ldots, 7$ and $\operatorname{sortInde}_{i}=\{1,1,2,1,3,2,1\}$ and sortIndex $_{j}=\{1,2,1,3,1,2,4\} \ldots \ldots \ldots \ldots \ldots$

A.4 Polynomial chaoses and their variances for a second-order and three-dimensional $\mathrm{PCE}[1] \ldots \ldots \ldots \ldots \ldots 1 . \ldots \ldots \ldots \ldots$

A.5 The expectation and variance of second-order and three-dimensional PC basis [2].195

B.1 Nodes and weights for Gauss-Hermite quadrature in one dimension and third level. . . . . . . . . . . . . . . . . . . 207

B.2 Nodes and weights for Gauss-Hermite quadrature in two dimension and third

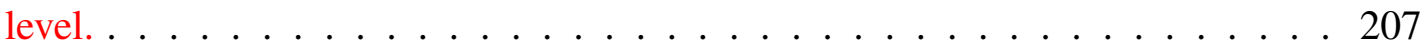




\section{List of Algorithms}

1 : Parallel PCGM Algorithm . . . . . . . . . . . . . . . . . . 86

2 : Stochastic Finite Element Assembly for DD Blocks . . . . . . . . . . . . 87

3 : NNC-PMVP: Parallel Mat-Vec Product Procedure . . . . . . . . . . . . . . . 89

4 : NNC-PP: Two-Level Parallel Preconditioning Procedure . . . . . . . . . 90

5 : LNS: Local Neumann Solve Procedure ． . . . . . . . . . . . . . . . . . 90

$6 \quad$ : LMVP : Local Mat-Vec Product Procedure . . . . . . . . . . . . . . . 91

7 : GCP: Global Coarse Solve using coarse-PCGM . . . . . . . . . . . . . 92

8 : Sc-PMVP: Parallel Schur-Mat-Vec Product Procedure . . . . . . . . . . . . . 93

9 : L-PP: Lumped Parallel Preconditioning Procedure . . . . . . . . . . . . . 93

10 : FETI-DP-PMVP: Parallel Mat-Vec Product Procedure . . . . . . . . . . 95

11 : D-PP: Dirichlet Parallel Preconditioning Procedure . . . . . . . . . . . . 96

12 : Stochastic System Matrix Assembly Procedure . . . . . . . . . . . . . . . 198

$13:$ NISP Procedure . . . . . . . . . . . . . . . . . . . . . . . . . 209 


\section{List of Acronyms}

BDDC Balancing Domain Decomposition

BDDC Balancing Domain Decomposition by Constraints

BiCG Biconjugate Gradient Method

BiCGSTAB Biconjugate Gradient Stabilized Method

BJ Block-Jacobi

CFD Computational Fluid Dynamics

CGM Conjugate Gradient Method

CoV Coefficient of Variation

DD Domain Decomposition

DDM Domain Decomposition Method

FETI Finite Element Tearing and Interconnect

FETI-DP Dual-Primal Finite Element Tearing and Interconnect

FEM Finite Element Method

FLOPs Floating Point Operations Per Second

FSI Fluid-Structure Interactions

GCP Global Coarse Problem

GMRES Generalized Minimal Residual Method

GPU Graphics Processing Unit

GSA Global Sensitivity Analysis

HPC High-Performance Computing

KLE Karhunen-Loève expansion 
KSP Krylov Subspace Method

LMVP Local Matrix-Vector Product

LNS Local Neumann Solve

MCS Monte Carlo Simulations

MPI Message Passing Interface

NISP Non-Intrusive Spectral Projection

NN Neumann-Neumann

NNC Neumann-Neumann with Coarse Grid

PCE Polynomial Chaos Expansion

PCKF Polynomial Chaos Kalman Filter

PCGM Preconditioned Conjugate Gradient Method

PDE Partial Differential Equation

PDF Probability Density Function

PMVP Parallel Matrix-Vector Product

PP Parallel Preconditioner

RAM Random Access Memory

RVs Random Variables

SC Stochastic Collocation

SFEM Stochastic Finite Element Method

SG Stochastic Galerkin

SGFEM Stochastic Galerkin Finite Element Method

SSFEM Spectral Stochastic Finite Element Method

UQ Uncertainty Quantification 


\section{Chapter 1}

\section{Introduction}

The only certain aspect of a numerical simulation of a physical phenomenon is that it invariably involves errors and uncertainties [3]. It is therefore essential to (a) verify and validate the accuracy of numerical predictions of computer simulations and (b) quantify the level of confidence in these numerical predictions. Although verification, validation, and uncertainty quantification (UQ) of simulations models have been subjects of extensive investigations for several years, their further development remains essential to have a significant impact on the reliability and utility of numerical simulation in the future [4-8].

The spectral stochastic finite element method (SSFEM) $[1,2,9,10]$ developed based on homogenous chaos theory of Wiener [11] and by leveraging the advantages of deterministic finite element method (FEM) has been successfully applied for the UQ of many engineering applications [12-16]. High-level framework of uncertainty propagation for stochastic PDEs using intrusive SSFEM is illustrated in Fig. 1.1. In this framework, the procedure is divided into the following steps. Step 1 involves specification of the probabilistic model, i.e., a stochastic PDE. In Step 2, the random system parameters are characterized using Karhunen-Loève expansion (KLE) and polynomial chaos expansion (PCE) $[1,2,9,10]$. The FEM is used in the spatial domain and PCE is used in the stochastic domain to discretize the stochastic PDE using intrusive SSFEM, resulting in a large-scale system of linear equations $[1,2,13]$. Then, linear system 
solvers are employed to solve the system for PCE coefficients of the solution process [17-19]. In Step 3, the stochastic response is analyzed in terms of mean, variance, higher-order statistics and joint probability density functions of the output using PCE coefficients of the solution process. The output (prior) PCE coefficients can also be used for data assimilation, for example, using polynomial chaos Kalman filter (PCKF) to obtain the updated (posterior) PCE coefficients by blending available measurements [20,21]. Furthermore, the PCE coefficients can be used for global sensitivity analysis (GSA), i.e., quantifying the respective effects of input random parameters on the variance of the output quantity of interest [22].

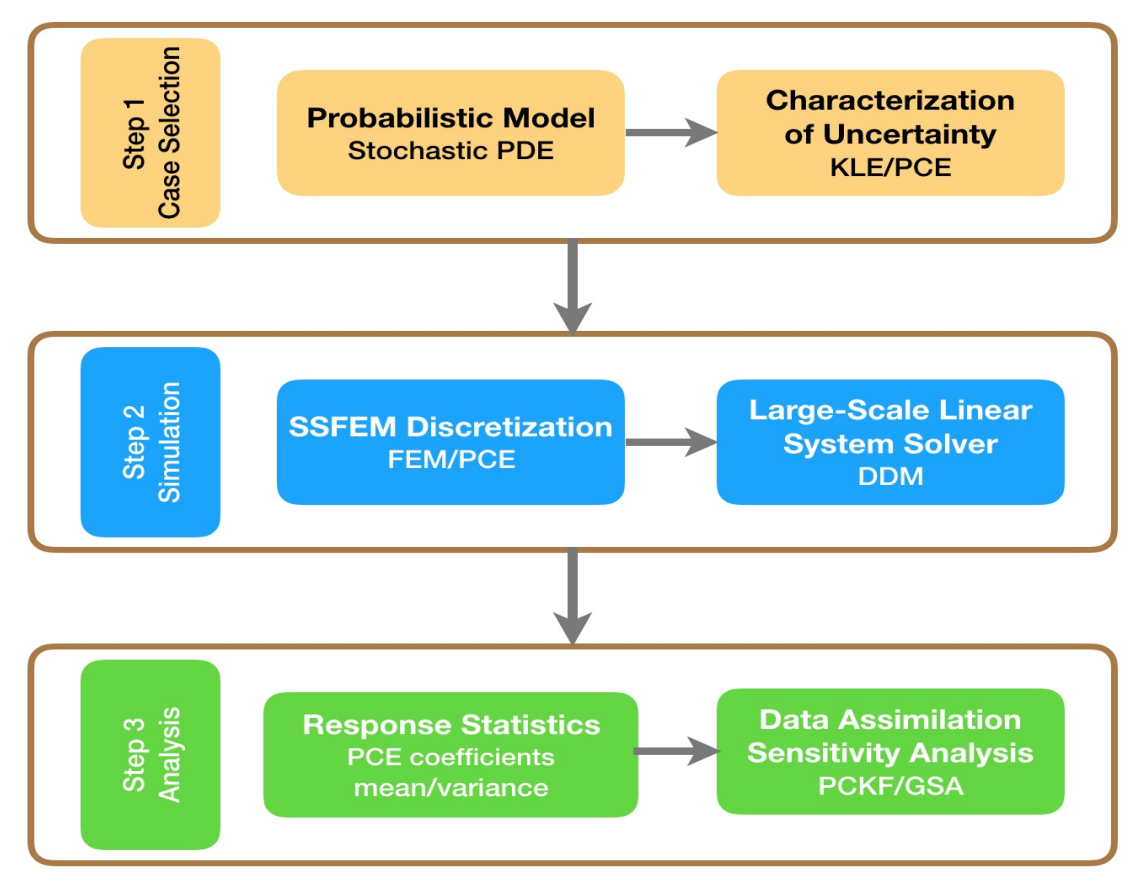

Figure 1.1: Uncertainty propagation framework for large-scale stochastic PDEs.

The stochastic modeling of practical engineering systems using SSFEM may involve solving a system of equations with $10^{6}-10^{9}$ of unknowns, rendering it a task of exploiting highperformance computing (HPC). This is the step 2 in the uncertainty propagation framework outlined in Fig. 1.1; and it is the most critical step in the whole process. To efficiently exploit modern HPC platforms, it is essential to design parallel algorithms tailored to fit these applications. Domain decomposition method (DDM) can provide the necessary platform to develop fast and efficient solvers for large-scale stochastic systems [17-19, 23, 24]. 
Considerations such as these motivated Sarkar et al. [23] to develop the theoretical formulation for iterative substructuring methods for stochastic PDEs. Subsequently, Subber and Sarkar $[17,18]$ formulated and employed one-level and two-level DDM solvers for stochastic PDEs with a few random variables. Taking motivation from their work, in this thesis, the primary attention is given to the development and efficient parallel implementation of scalable DD solvers for two and three dimensional elliptic stochastic PDEs with a large number of random variables. For three-dimensional coupled stochastic PDE systems, the extended wirebasketbased coarse grid is developed to improve the performance and scalability aspects of these solvers $[25,26]$. Furthermore, the DD solvers developed for intrusive SSFEM are shown to outperform the non-intrusive SSFEM approach with the sparse grid quadrature in Sec. 3.3.6. This fact highlights the advantages of the intrusive approach and emphasizes the necessity of the scalable solvers and high-performance computing in uncertainty quantification.

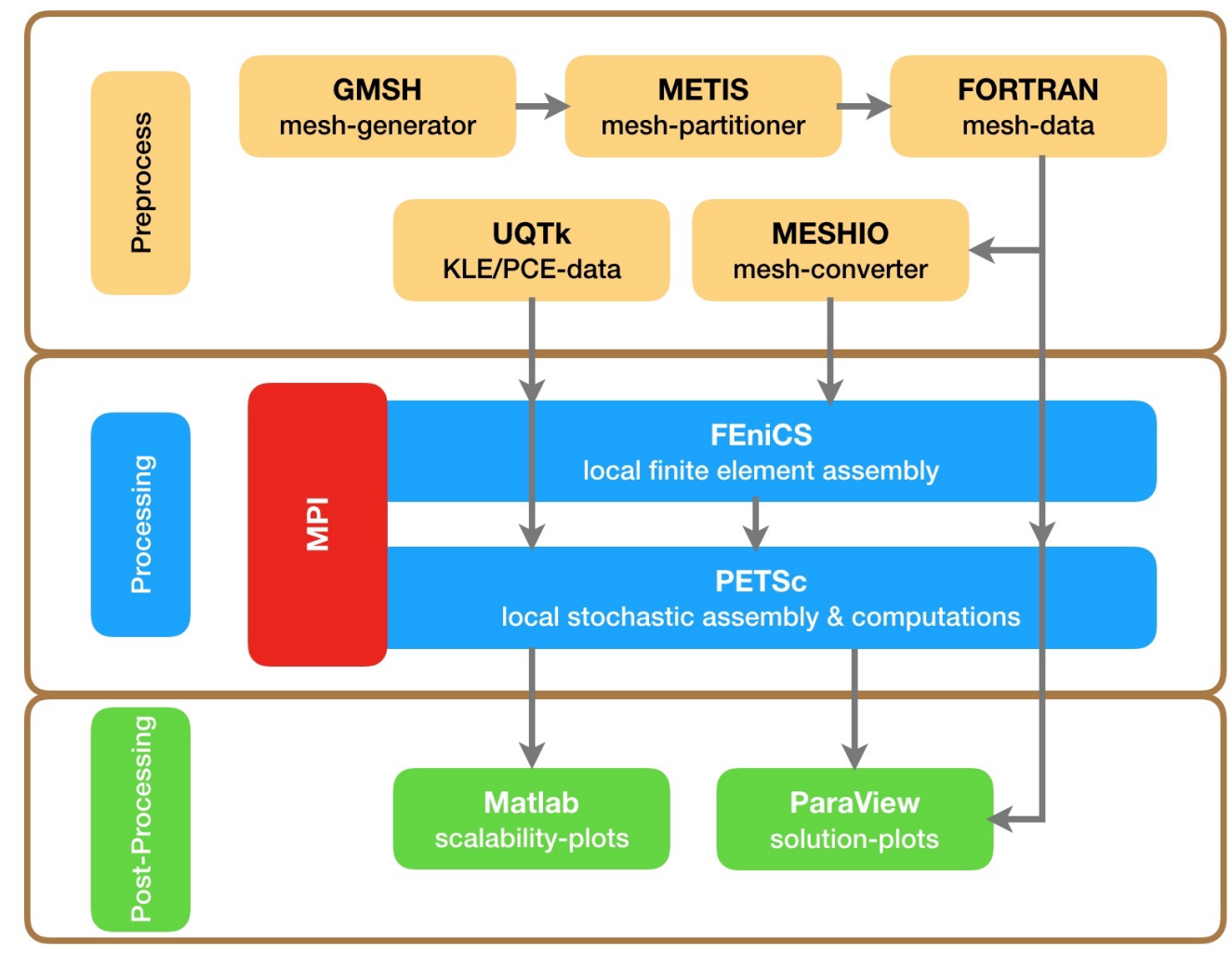

Figure 1.2: Implementational framework of DD-based SSFEM package for large-scale discretized stochastic PDEs in high-performance computing. 
Fig. 1.2 illustrates high-level implementation framework and work flow of DD-based SSFEM solvers for stochastic PDEs, employed in this thesis. The DD solvers for stochastic PDEs are implemented using external HPC libraries whenever appropriate. The work flow is divided into three steps, i.e., preprocessing, processing and post-processing. The preprocessing step primarily involves preparing data (KLE/PCE and mesh) for the solver. Various external scientific computing packages, such as, GMSH [27], METIS [28], UQTk [29] and MESHIO [30] are utilized in this step. In the main processing part, which is computationally intensive, PETSc [31] based sparse data-structures and computationally efficient routines are employed for memory optimization and linear algebraic calculations. Furthermore, the solvers are coupled with the FEniCS [32] general purpose deterministic finite element package, to simplify the application to a variety of PDEs. Message Passing Interface (MPI) [33] communication routines are employed for interprocessor communication. For post-processing, ParaView [34] and Matlab [35] are used.

\subsection{Background}

Numerical modeling and computer simulations have emerged as a powerful tool that is revolutionizing the way we design and analyze technological and scientific devices. It has become a crucial approach for resolving a multitude of scientific and engineering problems [6,36-38]. Numerical modeling and computer simulations have gained popularity for the following reasons as summarized in the report by National Science Foundation [6]:

- It provides a compelling alternative to expensive, complicated, and sometimes even impossible experiments [37].

- It enables us to design and manufacture materials and engineering devices on a more scientific basis with less trial and error, which ultimately reduces the number of design cycles.

- It improves the ability to predict the outcomes before allocating resources to specific design 
and therefore significantly save the design cost.

- It expands our ability to cope with the problems that have been too complex for traditional methods and beyond the reach of experimental science [7].

- It has become an essential complement to experiments for the study of physical phenomena and the design of experimental set-ups and test matrix.

Numerical simulations are used for large-scale problems with ever increasing complexity in almost every discipline of science and engineering [5]. Therefore, as stated previously, it is crucial to know how accurate they are and what is the level of certainty in their predictions [4]. Although many tools are developed to answer such questions, it remains difficult to provide confidence level in the quantitative information obtained from numerical simulations $[6,39,40]$. This is mainly due to the presence of uncertainty in the numerical modeling of numerous scientific and engineering systems. Hence, to realistically predict the behavior of such systems, the effects of uncertainty must be considered rationally [41-44]. Although uncertainty quantification of numerical simulations has been the subject of study for many years [45-47], its further development is essential to have a profound impact on the reliability and utility of simulation methods in the future $[4,6,48,49]$.

Every numerical model designed to simulate a physical phenomenon is bound to have errors and uncertainties [42,50-52]. The error in the numerical simulation is a recognizable deficiency and hence, deterministic by nature. It arises mainly due to numerical approximation or discretization of the problem [53]. Error can be controlled by using a high resolution numerical models $[42,45,54]$. On the other hand, uncertainty is a potential deficiency which relates to the physics of the problem [53]. It appears due to lack of knowledge and inherent randomness in nature. However, uncertainty can be characterized and quantified to a certain extent [1,4,42,44, 48, 55-57]. 
Uncertainties present in the numerical modeling of scientific and engineering applications are generally categorized into two parts: (1) aleatory uncertainty, also referred as inherent, objective or irreducible, and (2) epistemic uncertainty, also called subjective or reducible [42, 47, $52-54,58]$. In the current work, we have focused on the aleatory or inherent uncertainty. These uncertainties enter the computational problem through random system parameters, initial and boundary conditions and/or random external forcing $[42,47]$. The science of quantitative characterization and reduction of uncertainties is called uncertainty quantification (UQ). Characterizing uncertainty in numerical models increases the confidence level in our design and provides the basis for certification in high-consequence decision-making process [42, 43, 53, 55]. Also, UQ can be used to provide the confidence interval or to add error bars on numerical predictions $[59,60]$. Uncertainty quantification is commonly achieved through computer experiments using a numerical model of the underlying physical phenomenon $[43,44,48,50]$.

Probabilistic methods for UQ in science and engineering can be broadly classified into the sampling and sample-free approaches [9,61-64]. The most commonly used approach for UQ is Monte Carlo sampling (MCS) [65,66]. This technique is popular among engineers and scientist mainly because of its robustness and simplicity in implementation [39,40,67]. However, the rate of convergence of MCS is highly dependent on the ensemble size, therefore, becomes impractical or infeasible for large-scale applications owing to excessive computational cost. These challenges motivated the search for alternative approaches, such as perturbation approach $[1,68-70]$ and Neumann-series expansion $[1,63,70]$. However, these methods have their own limitations and are also not practical for the large-scale applications [1,9,63,70-72]. This motivated the development of polynomial chaos expansion (PCE) based spectral projection methods [1,9,64,71]. The brief overview of the PCE based spectral stochastic finite element methods is presented in the next sections. 


\subsection{Brief Overview of Spectral Stochastic Finite Element Methods for Uncertainty Quantification}

Physical phenomena governed by a partial differential equation (PDE) can be modeled using the finite element method (FEM) [73]. It is one of the widely employed numerical methods for solving problems in science and engineering. However, the FEM is deterministic by nature. Therefore it cannot be directly applied to stochastic PDEs [74-76]. To surmount this issue, several strategies have been developed to model stochastic PDEs using finite element methods. These techniques are collectively referred to as stochastic finite element methods (SFEM) [1,9, 64, 74,75]. SFEM can be broadly categorized into (a) sampling based approaches $[9,62,67,77-$ 82] and (b) sampling-free approaches [1, 61, 64, 71, 83-86].

Monte Carlo (MC) sampling based SFEM is the long-established technique for uncertainty quantification of stochastic PDEs $[65,66,87]$. This method involves generating a large set of independent realizations of the solution process based on the distribution of input random system parameters and then characterizing the response statistics based on those realizations [77,87]. The rate of convergence of MCS is independent of the number of uncertain parameters, but it is highly depended on the ensemble size. Hence, to achieve a good level of accuracy, a large number of realizations $[77,87]$ are needed. The overwhelming computational cost and the other limitations of traditional UQ approaches motivated the development of spectral stochastic finite element method (SSFEM) based on polynomial chaos expansion (PCE) [1, 9, 63, 64].

The SSFEM has proven to be an efficient alternative of MCS based SFEM for many engineering and scientific applications $[1,88,89]$. It has been successfully applied in a wide variety of fields, which includes computational structural mechanics (CSM) [2, 10,43, 72,90-92], computational fluid dynamics (CFD) [9, 13, 56, 88,93-97], fluid-structure interactions (FSI) [98-100], structural dynamics and aeroelasticity [15,60,101-105]. In the SSFEM approach, the uncertain system parameters and the solution process are represented by employing orthogonal polynomials as the basis in the random dimension [1]. Based on the procedure followed to obtain 
the expansion coefficients of the solution process, the SSFEM methods are mainly classified into two categories, (a) the non-intrusive (sampling) approach [79,80,106] and (b) intrusive (sample-free) approach commonly referred to as stochastic Galerkin method $[61,71,86]$.

In the context of non-intrusive spectral projection (NISP), a type of non-intrusive SSFEM [9, $79,107]$, the samples of the deterministic solutions are used to compute the expansion coefficients of the solution process. First, the response is projected on each basis function using inner products. Then, by utilizing the polynomial orthogonality properties, the unknown coefficients of the solution process are obtained. The inner products appearing in this context involve multidimensional integrals which can be evaluated utilizing MC sampling, tensor product quadrature or sparse grid quadrature rules [9,79, 106, 108, 109]. Another widely employed non-intrusive technique is the stochastic collocation (SC) method [78, 80, 81, 110]. In the SC approach, the Lagrange interpolation polynomials are employed to approximate the solution process. A set of prescribed structured collocation points, defined by a tensor grid or sparse grid, is used to construct the basis of the polynomials $[62,111,112]$. The coefficients of the polynomial are computed as the solution at the collocation points [78, 113,114]. The implementation of both the non-intrusive approaches, NISP and SC methods, involves repeatedly solving deterministic problems corresponding to realizations of the random variables at the quadrature points or collocation points $[78,106,111]$. Hence, in these approaches, one can employ readily available deterministic solvers for simulations [106,113,115].

On the other hand, in the context of the intrusive SSFEM approach, commonly referred to as stochastic Galerkin finite element method (SGFEM), the functional expansion of uncertain system parameters and solution process using PCE are incorporated into the governing equation $[1,2,88]$. Then, performing Galerkin projection onto the space spanned by orthogonal polynomial basis, the original stochastic model is transformed into a set of coupled deterministic equations [2]. The resulting system is solved for the PCE coefficients of the solution process. Therefore, implementation of SGFEM demands intrusive adjustments in the PDE solver for numerical simulation $[104,106,113]$. 
Among these two, the non-intrusive approach is widely adopted for uncertainty quantification in both academic and industrial applications. This is because the non-intrusive methods can utilize the existing deterministic codes as a black box $[9,106,108]$. On the contrary, the intrusive approach requires a new simulation code which might be a tedious task to undertake. However, as reported in the literature, the intrusive approach has the following advantages over the non-intrusive methods:

- To achieve the same level of accuracy, the non-intrusive methods may require simulations at a large set of sample points or collocation points. In such cases, it can involve more computational cost due to repeated system solves than that of the intrusive approach $[106,113,115]$.

- In the intrusive approach, the PCE coefficients of the solution process are solved as a coupled system of equations. Therefore, the error due to the finite representation is minimum leading to optimal accuracy $[106,115]$. On the contrary, there is no explicit control of the error in the non-intrusive methods except at the sampling points or collocation points where the error is minimum. Therefore the accuracy of the solution in non-intrusive approach highly depends on the selection of the sampling or collocation points $[9,115,116]$.

- If efficient solvers are employed, for the same accuracy level, the intrusive approach can provide the solution much faster than the non-intrusive approaches in the cases of a large number of RVs [106]. The performance comparison between the intrusive and non-intrusive approaches, along with other available solution methods for SFEM, is debated by many researchers as evident from the several articles available in the literature [72,106,116-119]. 


\subsection{Brief Overview of Domain Decomposition Methods for Uncertainty Quantification}

The computational cost of SGFEM is dependent on (a) spatial discretization $(N)$ i.e., the number of nodes (plus the degrees of freedom per node) in the finite element mesh and (b) stochastic discretization $(P)$ i.e., the number of PCE terms, which is function of the order and dimension of functional expansion used to represent the solution process $[1,120]$. For example, consider an adequately dense finite element mesh with $N$ nodes such that the set of equations arising from deterministic finite element discretization can be simulated using a conventional desktop computer without exceeding its RAM (random access memory). For uncertainty quantification, as we introduce the $P$ stochastic discretization terms, the size of the global system resulting from SGFEM setting grows to $N \times P$. Consequently, we can conceivably no longer fit the new problem in the memory of the same desktop computer. In such situations, to compute the solution, the spatial domain (i.e. finite element mesh) can be divided into $s$ subdomains of size $N_{s}$ using domain decomposition methods (DDM) [121-124]. Then each subdomain level system, in this case, $N_{s} \times P \approx N$, can be solved using a computing cluster with $s$ computing cores each of which is similar to that of the conventional desktop computer. Further increase in stochastic dimensions can be accommodated by dividing the physical domain into more subdomains and accordingly using more computing cores to solve the problem. Therefore, the DDM can help us to accommodate increased problem size due to increase in stochastic dimensions [19, 23, 24, 125-127].

In an arbitrary setting, the domain decomposition (DD) is necessarily the same in every field of science and engineering, yet it is perceived differently in each field. For example, in the context of multiphysics problems, domain decomposition may result naturally from partitioning the physical region into subregions based on phenomenological considerations [125,128]. From the algebraic point of view, DD is described as a parallel algorithm to divide and solve the large scale system of equations [124,128]. In the parallel computing perspective, DD is viewed as a 
process of distributing a large data set into a number of smaller groups which can be processed concurrently using distributed memory architecture computers [124, 129, 130]. The DDM have found its utilization in various scientific disciplines including structural mechanics [131-134] and computational fluid dynamics [135-138].

In general, the domain decomposition methods are classified into two groups, (a) overlapping methods and (b) non-overlapping methods [125,128]. Overlapping domain decomposition methods are some of the earliest methods and are viewed as a generalization of block Jacobi or block Gauss-Seidel methods [125, 128]. In the context of overlapping DDM commonly referred as Schur complement or sub-structuring methods, smaller problems are alternately solved on each of the overlapping subdomains and then propagate this interior solution onto the artificial boundary [124, 128, 136]. There are two popular variants in this category: (a) additive Schwarz methods and (b) multiplicative Schwarz methods. The detailed theory, formulation, and applications of overlapping DDM can be found in the following articles and the references therein $[125,128,137]$.

On the other hand, in the non-overlapping DD methods, the problem is formulated on each subdomain separately, and then the local degrees of freedom are divided into interior and interface based on their locations. The global assembly is performed using those subdomain level (local) variables, followed by algebraic manipulation based on Gaussian elimination; the original global system is reduced to the Schur complement system [124, 128, 133]. Subsequently, the global interface unknowns are computed by solving the Schur complement systems and then the local interior unknowns are obtained by solving the local interior system simultaneously on each subdomain $[128,133,139]$. Depending on the procedure employed to solve the Schur complement system, the non-overlapping DDM are classified into the direct substructuring and the iterative substructuring methods [128, 133, 139].

In the direct substructuring method, the Schur complement system is solved using direct solvers. This method is effective for a small linear system with few unknowns but computationally expensive for a large-scale system with millions of unknown. For instance, a coupled 
system of linear equations appear in the context of intrusive SSFEM are extremely large [17,23]. Therefore, the iterative substructuring methods are preferred in the stochastic mechanics community [24, 140-143]. The iterative substructuring techniques are further categorized into the primal and dual methods $[132,139]$. In the primal methods, the continuity conditions are strongly enforced by global assembly along the interfaces $[24,132,139,141]$. This category contains Neumann-Neumann (NN) [24, 134], balancing domain decomposition (BDD) [123, 144], balancing domain decomposition by constraints (BDDC) and their variants [123, 141, 145]. On the other hand, in the dual methods, the continuity conditions are weakly enforced by Lagrange multipliers along the interfaces [146-148]. This category includes the finite element tearing and interconnect (FETI) and the dual-primal finite element tearing and interconnect (FETI-DP) and its variants [131, 147, 149].

The system formulated using primal, dual or dual-primal substructuring approach can be solved using Krylov subspace methods (KSP) [150,151]. The KSP are prominent iterative methods primarily based on the projection process onto Krylov subspace [150]. For the cases where the system matrix is symmetric, for example, in the finite element discretization of the diffusion equation, the conjugate gradient (CG) methods are usually applied [150]. In the cases of unsymmetric system matrices, for instance, resulting from the finite element modeling of convection (advection)-diffusion equation, the methods commonly used are generalized minimal residual method (GMRES), biconjugate gradient method (BiCG) or biconjugate gradient stabilized method (BiCGSTAB) [150].

The performance of the KSP methods can be greatly improved by using preconditioners [150, 151]. The enhanced CG method is called preconditioned conjugate gradient method (PCGM) and the modified BiCG is called preconditioned BiCG and so on [150]. Preconditioning is a very significant component of the iterative methods for the large-scale applications. Preconditioning enhances both the robustness and the efficiency of the iterative solvers. The application of preconditioner effectively reduces the condition number of the system and accelerates the convergence of the solver [140,142, 143, 150]. 
In the past, various preconditioners were used to accelerate the performance of the iterative solvers for the SGFEM [10,17,90,127,148,152-157]. The stochastic FE matrix resulting in SSFEM is usually diagonally dominant and composed of blocks of deterministic FE matrices $[10,90]$. This fact motivated the use of block-Jacobi (BJ) preconditioners $[10,149,153]$. The performance of the BJ preconditioners is found to be sensitive to the level of uncertainty in the system [10,90,153]. The Kronecker product preconditioner, introduced by Ullmann [154] to exploit the block symmetric structure of system matrix, arises in the context of SSFEM. It is shown to enhance the performance of iterative solvers in comparison with the mean based preconditioners. Bedrich et al. [155] implemented the hierarchical Schur complement preconditioner to exploit the recursive hierarchy in the structure of the global stochastic finite element matrices. The Schwarz domain decomposition method is also used as a preconditioner for iterative methods $[128,137,156]$. However, the general Schwarz additive methods are one-level techniques and hence their performance deteriorates as the number of subdomains increases $[128,137,139]$. The two-level additive Schwarz preconditioner employed for the iterative solution of the SSFEM is shown to improve performance over the one-level methods [156].

Furthermore, Subber and Sarkar [17,24] implemented Neumann-Neumann (NN) preconditioners with PCGM solver to tackle the resulting system of linear equations in the setting of intrusive SSFEM. It is found to be more effective compared to simple lumped and weighted lumped preconditioners [24]. However, the performance of one-level NN preconditioner deteriorates as the number of subdomains increases [17]. To surmount this issue, the authors also applied two-level Neumann-Neumann with coarse grid (NNC) preconditioner [17, 140]. The vertex-based coarse grid used in this method provides the mechanism for global communication which effectively improves the convergence compared to one level preconditioners [17,24]. Numerical experiments performed on the scalar-valued stochastic PDEs demonstrate that the NNC based PCGM solver is numerically scalable with spatial problem size and number of subdomains in the case of a small number of random variables [17,24]. 


\subsection{Brief Overview of High-Performance Computing in Uncertainty Quantification}

The remarkable growth in the field of numerical modeling and computer simulations is propelled by the rapid advances in computing platforms and the widespread availability of high-end computing facilities $[5,38]$. High-performance computing (HPC) has become a key technology which provides the ability to solve large-scale real-world applications [37,38]. HPC in itself is an interdisciplinary research field in computer science and applied mathematics. It primarily involves developing algorithms and software to exploit high-performance computing clusters for large-scale numerical simulations [5, 6]. From the hardware perspective, HPC involves developing computing platforms using advanced technologies that can, for instance, solve multiple problems in parallel with high efficiency by consuming low power [8, 158]. It also includes developing efficient communication technologies necessary to employ the large-scale computing clusters to tackle a given problem in parallel $[158,159]$. In HPC the key notion is to improve parallel scaling, i.e. the ability to improve the performance of a given solver by increasing the number of cores and memory [6,38,160,161].

In SSFEM framework, the solution of a stochastic PDE is obtained by either the intrusive or non-intrusive approach. The sampling based non-intrusive SSFEM methods can be easily parallelized as each sample can be simulated independently. Such algorithms which are intrinsically parallel are often called embarrassingly parallel [161,162]. However, the SSFEM models with high-resolution demand large memory (RAM). Therefore each sample itself needs to be processed in parallel affecting the intrinsic embarrassingly parallel behavior of the non-intrusive approach. Also, in the cases of high stochastic dimension, a large number of such samples needs to be simulated. Therefore, the sampling based approaches might not be the optimal choice for such problems $[113,161]$. On the other hand, in the intrusive SSFEM, the coupled sets of equations obtained by incorporating PCE of the random system parameters and the associated solution process into the governing equations are solved simultaneously [18,90,161]. From the 
implementational perspective, the intrusive approach using HPC requires the exchange of data among computing nodes in order to solve the given problem [18,19]. Therefore, the solution of the intrusive SSFEM system requires considerable efforts in terms of algorithmic development and HPC implementation [18, 19, 161].

The development of next generation HPC algorithms for UQ [161,163] demands scalability and compatibility with the current HPC systems and adaptability with the future exascale computing platforms [164-166]. It is believed that to achieve the exaFLOPs, i.e. $10^{18}$ floatingpoint operations per second (FLOPs), we need to build clusters with millions of computing cores $[164,165]$. The utilization of SSFEM for large-scale industrial applications are the ideal problems to exploit emerging exascale computing $[161,163]$. It is primarily because with increasing stochastic dimensions the size of the problems needed to be handled in the intrusive approach significantly increases and the number of sample evaluations grows rapidly in the cases of non-intrusive approaches rendering them impractical [19, 161,163].

The new algorithms for extreme-scale UQ applications need to scale with emerging computing clusters with millions of cores. Domain decomposition methods are shown to be suitable algorithms for SGFEM $[18,19]$. The DD algorithms with efficient preconditioners are ideal for parallel implementation and are shown to scale with increasing problem size and the number of cores $[18,19,161]$. In the DD formalism, as the problem size grows, we can divide the physical domain into smaller subdomains and use more cores to solve the new problem [19]. As a result, the scalable DD algorithms with the emerging HPC platforms can assist us to tackle ultra resolution numerical models with a large number of random variables, thereby significantly reducing the spatial and stochastic discretization errors [19,24,42,55]. 


\subsection{Literature Review of Solution Methods for Intrusive Spectral Stochastic Finite Element Methods}

This thesis is primarily focused on the development of scalable parallel solvers for the coupled system of equations in the context of intrusive SSFEM. Therefore, in this section, we will briefly review the various methods employed in the past to tackle the system of linear equations in the setting of intrusive SSFEM. The system matrix obtained in the setting of intrusive SSFEM has a block sparsity structure as shown in Fig. 2.3. Each block of the system matrix has similar sparsity pattern to that of a deterministic FEM. Therefore, the sparse direct solvers can be employed to solve the resulting system of equations [10,167]. However, in the cases of intrusive SSFEM for large-scale system focused in this thesis, the sparse direct solvers demand more memory compared to iterative solvers. Thus, in the past, more attention has been given to the development of the sparse iterative solvers with the suitable preconditioners [10,90,150,168]. The preconditioned intrusive SSFEM system can be written as,

$$
M^{-1}[\mathscr{A}]\{\mathcal{U}\}=M^{-1}\{\mathscr{F}\} .
$$

where $M^{-1}$ denotes the preconditioner.

Ghanem and Kruger (1996) [10] used preconditioned conjugate gradient method (PCGM) with the block-Jacobi (BJ) preconditioner to solve the system of equations in SSFEM. The blocks of the preconditioner are obtained by LU factorization of the mean stiffness matrix. Another solution procedure based on hierarchical basis concepts was also proposed. In the second method, the intrusive SSFEM system is solved using the PCGM with an incomplete Cholesky factorization preconditioner. The performance of the PCGM equipped the BJ preconditioner is improved compared to the traditional Jacobi preconditioner. However, the number of PCGM iterations required for the convergence grows as the number of PCE terms increases. 
Pellissetti and Ghanem (1996) [90] iteratively solved the intrusive system of equations arising using PCGM with a BJ preconditioner. They used the approximate inverse of each block of the matrix instead of LU decomposition. Taking the approximate inverse helps to preserve a prescribed sparsity structure, however, as the size of the matrix grows finding a good approximate inverse for the mean stiffness matrix is computationally expensive. Also, this preconditioner worked well for small random fluctuations, however for a high degree of uncertainty which requires a large number of PCE terms, the convergence rate of the $\mathrm{BJ}$ preconditioner deteriorates.

Le Maître et al. (2003) [169] used a multigrid method based on spatial coarsening to solve the linear system arising in the setting of SSFEM for a two-dimensional stochastic diffusion equation. The computational tests conducted to analyze the behavior of the solver suggest that the multigrid scheme exhibits a fast rate of convergence and good scalability concerning spatial resolution. However, the convergence rate drops as the order of the PCE increases and it is heavily dependent on the level of the coefficient of variation $(\mathrm{CoV})$ of the random system parameters. The convergence rate is slightly dependent on the correlation length of the stochastic diffusivity field, but it is very sensitive to multigrid parameters and cycle structure. Therefore, careful selection of these parameters is recommended.

Chung et al. [170] employed an incomplete block-Jacobi preconditioner with block GaussJacobi iterative method to solve the linear system in SSFEM. An element-by-element strategy is employed for the matrix-vector product required for the iterative solver. This strategy is less expensive concerning memory as it requires the storage of the element stochastic stiffness matrix. However, after each matrix-vector multiplication step, the resulting vector needs to be assembled which consumes a large computation time. They concluded that the performance of the solver is not significantly affected by increasing the order of PCE. However, increase in $\mathrm{CoV}$ of the random input parameters drives the iteration counts.

Elman and Furnival (2007) [152] solved the stochastic steady-state diffusion problem formulated using the multigrid method. The spatial discretization is varied from grid to grid while the stochastic discretization is held constant. The mesh parameter is varied from the coarse grid 
to fine grid. Performing theoretical and experimental analysis, they concluded that the convergence rate of the solver is independent of the mesh resolution similar to the deterministic cases. However, the performance of the solver for varying stochastic parameters such a $\mathrm{CoV}$ and the number of PCE terms was not performed.

Chao et al. (2007) [171] employed a black-box additive Schwarz domain decomposition preconditioner with a recycling Krylov subspace method to solve the linear system of equation in intrusive SSFEM. Double orthogonal basis are used to decouple the probabilistic space resulting from a sequence of uncoupled systems of linear equations. They conclude that the preconditioner achieves a scalable performance concerning the number of subdomains and finite element mesh size. The recycling technique employed assists in reducing the total execution time. However the decoupling procedure is only applicable for the case where the input random variables in the system parameters are independent.

Rosseel et al. (2008) [151] used the algebraic multigrid method to tackle the linear system arising in the context of SSFEM. The hierarchy of meshes required for the multigrid method is built by using the mean stiffness matrix. The number of multigrid iterations is shown to be independent of the geometric mesh size for Gaussian and non-Gaussian material properties. In the case of uniform probability density function (PDF), the convergence rate of the proposed multigrid solver is proven to be asymptotically independent of the order of PCE. However, for a Gaussian PDF, it does not show similar behavior.

Powell and Elman (2009) [153] utilized BJ preconditioner on the algebraic multigrid method to achieve optimal computational complexity. They provided a theoretical basis for the BJ preconditioner and established a bound for the condition number and highlight that these condition number bounds depend on the order of PCE, the number of random variables and coefficient of variation of the random system parameters. However, the performance of the algebraic multigrid scheme is shown to be independent of the size of the spatial discretization.

Ghosh et al. (2009) [148] used the block-diagonal preconditioner and its FETI-DP solver tailored for systems with multiple and repeated right-hand sides. They employed a nested PCGM 
solver, where the outer PCGM for the full stochastic linear system is used and inner PCGM based solution for the individual block of the preconditioner is utilized. To speed-up the convergence rate of the inner PCGM, a recycling Krylov subspace method is utilized. Performance tests of the solver demonstrated good numerical scalability concerning the number of subdomains and the size of the problem.

Ullmann (2010) [154] proposed a Kronecker product preconditioner obtained by exploiting the block sparsity structure of the stochastic stiffness matrix. The proposed preconditioner for the linear system arising in the context of SSFEM showed improved performance compared to the mean-based preconditioner. A moderate increase in the iteration counts is observed when the level of uncertainty in the system parameters and the order of the stochastic expansion increase. Furthermore, the Kronecker product preconditioner cannot be parallelized entirely due to the presence of the off-diagonal block elements.

Sousedík et al. (2014) [155] devised hierarchical Schur complement preconditioner for the intrusive SSFEM. This preconditioner is formed by exploiting the recursive hierarchy in the structure of the global matrices. One of the submatrices in the two-by-two recursive hierarchical structure is block diagonal and one of the diagonal blocks in this submatrix is similar to the deterministic mean-value problem. The numerical experiments performed on an elliptic problem demonstrate the superiority of hierarchical Schur complement preconditioner concerning iteration count and floating point operations over the block version of the symmetric GaussSeidel method.

Subber and Loisel (2014) [156] used a two-level additive Schwarz preconditioner to iteratively solve the large-scale system of equations appearing in the context of intrusive SSFEM. It can be regarded as a generalization of the mean based BJ preconditioner. Their numerical analysis and computer experiments demonstrate that the convergence rate of stochastic Schwarz preconditioner is independent of the mesh parameters, the strength of randomness, dimension and order of the stochastic expansion in the cases of two-dimensional stochastic PDEs. Furthermore, the numerical simulations are performed to investigate condition number growth in 
regard to various system parameters.

Sarkar et al. (2009) [23] introduced the domain decomposition method for uncertainty quantification of stochastic PDEs. The theoretical framework presented is based on Schur complement based domain decomposition in the physical space and functional decomposition in the stochastic dimension. A direct substructuring technique is implemented to solve a steady state wave propagation problem in one-dimensional random media. A dense direct solver is used to tackle the stochastic Schur complement system. This approach gives the ability to exploit the deterministic domain decomposition algorithms in the context of stochastic PDEs.

Next, Subber and Sarkar (2013) [18] formulated a probabilistic dual-primal finite element tearing and interconnect (FETI-DP) method for the intrusive SSFEM in order to utilize HPC platforms for large-scale stochastic systems. In the context of stochastic FETI-DP method the dual interface operator contains a coarse problem. In the cases of two-dimensional stochastic PDEs the introduction of the coarse problem in the dual operator leads to a scalable performance of the FETI-DP method. The scalable performance of probabilistic FETI-DP solver is demonstrated for the stochastic PDEs, discretized using high mesh resolution and few random variables.

Furthermore, Subber and Sarkar (2014) [17] introduced a two-level Neumann-Neumann preconditioner (NNC) with vertex-based coarse grid to iteratively solve the intrusive SSFEM system. The implementation of vertex-based two-level Neumann-Neumann preconditioner involves solving a local problem on each subdomain and propagates information globally among the subdomains by solving a coarse problem. Note that, in this case the coarse problem is part of preconditioner as opposed to FETI-DP where the course problem is part of the operator. The vertex-based NNC preconditioner is shown to scale well for the cases with high mesh resolution and few random variables. However, for three-dimensional coupled stochastic PDE system, for instance, arising in the linear elasticity, both the NNC and FETI-DP solvers with the vertex-based coarse grid showed a poor numerical and parallel scalabilities [24].

In the recently published article by the author [19], the intrusive polynomial chaos expansion 
based two-level domain decomposition algorithms are extended to concurrently handle high resolution in both spatial and stochastic domains. Sparse iterative solvers with efficient preconditioners are employed to solve the resulting global and subdomain level local systems through three-level nested iterative solvers. An efficient stochastic assembly procedure is employed to directly assemble and store blocks of stochastic finite element matrix using deterministic assembly matrix blocks. Sparse data structures, routines and solvers from PETSc are employed to handle subdomain level algebraic matrix-vector product and system solves. The NNC/BDDC and FETIDP solver are shown to handle large-scale simulations with a large number of random variables.

Next, we list a few recently published articles closely related the current work. Silvester and Pranjal (2016) in [172] designed and implemented efficient solution algorithms for a symmetric positive-definite linear system arising in the context of stochastic Galerkin approximation of stochastic elliptic PDEs. They employed preconditioned minimal residual method with the incorporation of error control in the natural energy norm leading to a robust and optimally efficient stopping criterion. In their implementation, the iteration is terminated right after the algebraic error becomes insignificant compared to the approximation error.

Powell et al. (2017) [173] reformulated the system of equations arising in the setting of stochastic Galerkin finite element approximation of stochastic PDEs as multiterm linear matrix equations. They generalized the ideas from the rational Krylov subspace approximation to develop an efficient solution algorithm. A low-rank approximation to the solution matrix is determined by performing a projection onto a low-dimensional space to get an efficient solution strategy with the convergence rate independent of the spatial discretization. They conclude that the new strategy takes considerably less memory than the standard mean-based PCGM applied to the Kronecker formulation of the linear systems.

Stavroulakis et al. (2017) [174] employed graphics processing units (GPU) based domain decomposition solution for spectral stochastic finite element method. They demonstrate the benefits of employing the GPU capabilities in addressing intrusive SSFEM by using the dual 
domain decomposition method preconditioners tailored for the specific applications. Their results showed a significant enhancement in the computational performance as well as of the consumed energy efficiency in the spectral stochastic finite element method.

Pranesh and Ghosh (2018) [175] devised a parallel DD-based hybrid method combining MCS with SG approach for solving large-scale problems. In their approach, the dual-primal variant of the FETI-DP is chosen as the DDM followed by three distinct strategies of parallel implementation. The best approach is selected by performing numerical simulations to solve a linear elasticity problem in three dimensions with a large number of RVs.

\subsection{Problem Statement}

For the realistic simulations of a physical phenomenon governed by stochastic PDEs, it is essential to use (a) highly resolved meshes in the spatial domain to reduce the discretization error and (b) a large number of random variables in the stochastic domain to capture a wide range of random fluctuations in the input and output stochastic processes, in particular for parametric uncertainties. In the spectral stochastic finite element setting, the number of RVs dramatically influences the number of PCE terms required to represent the random system parameters and the solution process. Hence, incorporating PCE transforms the manageable deterministic model to an extreme-scale stochastic model. For instance, consider a two-dimensional unit square domain discretized using $N$ nodes in the physical space and $L$ RVs in the stochastic space. As we refine the problem in both physical and stochastic domains, the size of the intrusive SSFEM system increases significantly. For example, see Table 1.1 where $N$ is increased from 30,000 nodes to 200,000 nodes and $L$ from 3 RVs to 25 RVs while holding third order expansion which results in 20 to 3276 PCE terms, respectively. In those cases, the size of the system arising in the intrusive SSFEM setting grows from 0.6 million to 0.65 billion unknowns. Therefore, for a large-scale application, it becomes impossible to store the system matrix of that size in the RAM of a single computing node. 
In the cases of non-intrusive SSFEM, the number of deterministic systems solves $\left(N_{s}\right)$ needed to compute the solution coefficients increases with the number of random variables [1, 108]. For example, see Table 1.2 where $L$ is increased from $3 \mathrm{RVs}$ to $25 \mathrm{RVs}$ and the level of sparse grid quadrature, $Q_{l}$, from 3 to 4 . The number of quadrature points increases significantly, i.e. from 69 to 0.285 millions. This is a well-known problem in UQ community and it is commonly referred to as the curse of dimensionality $[63,72,176]$.

\begin{tabular}{lcccccc} 
Deterministic system size (FEM) & \multicolumn{5}{c}{ Stochastic system size in millions (SGFEM) } \\
\hline$N$ & $L=3$ & $L=5$ & $L=10$ & $L=15$ & $L=20$ & $L=25$ \\
\hline 30,000 & 0.6 & 1.7 & 8.6 & 24.5 & 53.1 & 98.3 \\
50,000 & 1.0 & 2.8 & 14.3 & 40.8 & 88.6 & 163.8 \\
100,000 & 2.0 & 5.5 & 29.0 & 81.6 & 177.1 & 327.6 \\
200,000 & 4.0 & 11.2 & 57.2 & 163.2 & 354.2 & 655.2 \\
\hline
\end{tabular}

Table 1.1: Intrusive SSFEM system matrix size with the number of RVs.

\begin{tabular}{lcccccc} 
Level of quadrature $\left(Q_{l}\right)$ & \multicolumn{6}{c}{ Number of quadrature points $\left(N_{s}\right)$} \\
\hline$Q_{l}$ & $L=3$ & $L=5$ & $L=10$ & $L=15$ & $L=20$ & $L=25$ \\
\hline 3 & 69 & 241 & 1581 & 5021 & 11561 & 22201 \\
4 & 165 & 781 & 8801 & 40001 & 120401 & 285901 \\
\hline
\end{tabular}

Table 1.2: Number of quadrature points with the level of quadrature.

Although we can easily simulate as many samples as needed, for the same level of uncertainty, the non-intrusive SSFEM approach demands the solution of a substantially larger number of equations $\left(N \times N_{s}\right)$ than the size of the linear system $(N \times P)$ resulting from the intrusive SSFEM approach [113,115]. For example, a mesh with $N=200,000, L=25$ and $Q_{l}=4$, leads to a problem size of 57 billions in the non-intrusive setting and for the same mesh with $L=25$ and the third order expansion, we are handling the problem with 0.65 billion unknowns in the intrusive setting. Therefore, the intrusive approach is computationally cheaper than the non-intrusive approach. However, developing solvers for intrusive SSFEM demands a lot more coding efforts compared to the non-intrusive SSFEM, where we can use an existing deterministic solver. Therefore, the non-intrusive approach is a more popular choice in the stochastic mechanics community $[106,162]$. 
From a HPC perspective, for the numerical models with a sufficient spatial resolution $(N)$ that fits into the local memory of a core, the non-intrusive sampling schemes become embarrassingly parallel. Here, each core can handle one realization. However, in the same model, if the spatial resolution increases $\left(N^{*}\right)$, a large number of cores are now engaged to tackle just a single sample. Therefore, the non-intrusive approaches lose the aforementioned parallelism. In the same scenario, the intrusive SSFEM in conjunction with domain decomposition methods and high-performance computing can be employed to tackle the resulting coupled system [17-19]. For example, as the problem size grows due to an increase in stochastic discretization and/or mesh resolution, the ensuing large-scale system can be divided into a number of smaller subsystems using domain decomposition and accordingly engage multiple computing nodes to handle those subproblems concurrently [17,19].

The intrusive PCE based non-overlapping DDM algorithms with preconditioned conjugate gradient method (PCGM) presented in $[17,18]$ showed excellent scalabilities for the cases with high mesh resolution and few random variables. However, for large-scale stochastic models with high-resolution discretization in both spatial and stochastic dimensions, these solvers suffer from (a) the large memory requirement in order to assemble and store the subdomain level stochastic finite element matrices and (b) the excessive computational effort needed in factorizing those local stochastic FE matrices [17,18].

For three-dimensional coupled (vector-valued) stochastic PDEs, for instance, arising in linear elasticity, the two-level BDDC/NNC and FETI-DP solvers with the vertex-based coarse grid constructed using only corner nodes showed poor numerical and parallel scalabilities [24]. Therefore, algorithmic developments are required to improve the BDDC/NNC and FETI-DP solvers performances for the cases of three-dimensional coupled stochastic PDE systems [24].

Furthermore, the DDM solvers developed in [17, 18,21] were never tested for robustness and scalability on a large-scale computing cluster with thousands of cores. In such cases, as we increase the number of subdomains, the global coarse problem arising in the construction of BDDC/NNC or FETI-DP solver becomes very large. As a result, the solution of the coarse 
problem can become the bottleneck. Therefore, for practical applicability of these solvers, it is necessary to demonstrate the scalabilities with respect to a large number of cores.

\subsection{Research Objectives}

Numerous realistic physical phenomena exhibit random fluctuations across many scales in the input and output processes. Models of these phenomena require stochastic PDEs. Capturing the fluctuations across a range of scales using numerical modeling of stochastic PDEs requires, among other things, high-resolution meshes in the spatial domain and a large number of RVs in the stochastic domain. In the intrusive SSFEM, using a large number of RVs in conjunction with a high-resolution mesh dramatically influences the size of the resulting system of equations. Therefore, forward uncertainty propagation of practical engineering applications using the intrusive SSFEM may involve solving a system of linear equations in the order of billions of unknowns. To enable the simulation of such numerical models, it is essential to address both algorithmic and implementational aspects together. Therefore, the goal of this research is to design scalable parallel algorithms for intrusive SSFEM that can efficiently utilize HPC.

Parallel iterative solvers with DD based preconditioners can effectively handle such systems. Although these algorithms exhibit excellent scalabilities for two-dimensional stochastic PDEs with a few RVs, significant algorithmic and implementational challenges exist to extending them to solve extreme-scale stochastic systems arising in the context of two and threedimensional stochastic PDEs with a large number of RVs. Furthermore, in the cases of threedimensional coupled stochastic PDE systems (for instance, in linear elasticity) existing DD algorithms with a simple vertex-based coarse grid lose scalability. Therefore, new algorithms with a better resolved coarse grid are needed. Such considerations motivated the development of the current work.

In this thesis, the primary attention is given to the development and efficient implementation of scalable solvers for stochastic PDEs with a large number of RVs for intrusive SSFEM. 
Thereby, this work essentially addresses the aforementioned curse of dimensionality issue in SSFEM up to a certain extent. The focus of the research is directed towards devising DD based scalable parallel solvers and their efficient HPC implementation for the scalar and vector-valued stochastic PDEs in two and three dimensions with a large number of random variables.

Another objective of this research is to demonstrate the superiority of intrusive SSFEM over non-intrusive SSFEM to tackle large-scale discretized stochastic PDEs with a large number of non-Gaussian RVs, hence, to emphasize the necessity of the scalable parallel algorithms and high-performance computing platforms for uncertainty propagation in large-scale engineering systems. This thesis also attempts to address the often criticized implementational challenges of the intrusive SSFEM which arise due to the necessity of intrusive adjustments. Therefore, this work explores the possibilities to generalize the applications of intrusive SSFEM to tackle a variety of PDEs.

\subsection{List of Contributions}

In this thesis, both algorithmic and HPC implementational aspects of two-level DD solvers for intrusive SSFEM are addressed for stochastic PDEs with a large number of RVs. The advances made in both algorithmic and implementational aspects assist in alleviating the curse of dimensionality issue in SSFEM. These advances also enable the DD-based intrusive SSFEM approach to outperform the commonly used sparse grid based non-intrusive SSFEM approach for high-dimensional stochastic PDEs.

The probabilistic wirebasket-based coarse grid for BDDC/NNC solver is devised to overcome the scalability issues of two-level DD solvers with vertex-based coarse grid in three dimensions [24, 139]. This development strengthens the mechanism for global error propagation and improves the scalability of the BDDC/NNC solver to tackle coupled stochastic PDE sys-

tems. Implementational challenges of the intrusive SSFEM arises due to the necessity of intrusive adjustments are addressed by coupling DD-based solvers with FEniCS general purpose 
FEM package. Therefore, this work generalizes the applications of intrusive SSFEM to tackle a variety of stochastic PDEs and emphasize the usefulness of the DD-based scalable solvers and high-performance computing for uncertainty propagation in large-scale engineering systems. To this end, the contributions are broadly categorized as follows.

- The probabilistic BDDC/NNC and FETI-DP solvers formulated in $[17,18]$ are extended in both algorithmic and implementation aspects to solve large-scale discretized stochastic PDEs with a large number of RVs. The three-level sparse iterative solvers which employ an efficient preconditioner at each level are implemented using MPI, PETSc, FEniCS and UQTk [19]. The algorithmic and implementation advancements enable the solvers to scale up to 25 random variables [19] as opposed to 4 random variables reported in [17,18]. Also, the maximum size of the SGFEM system with 216 million unknowns is solved using 1680 computing cores as opposed to the SGFEM system with only 8 million unknowns reported in $[17,18]$. Therefore, the improved solvers alleviate the curse of dimensionality in SGFEM and justify the applicability of the intrusive approach to large-scale engineering applications.

- The probabilistic BDDC/NNC and FETI-DP solvers using vertex based coarse grid developed in $[17,18]$ do not scale with the number of subdomains for three-dimensional elasticity problems [24]. In such cases, it is necessary to include additional constraints concerning interface-edges and interface-faces in the construction of the coarse grid to achieve scalable performance $[24,139]$. The wirebasket-based coarse grid developed for deterministic PDEs in [139] is extended here to tackle three dimensional stochastic PDE systems. The new coarse grid using the extended wirebasket include both corner nodes and interface-edges. This enriched coarse grid provides an efficient mechanism for global error propagation and thus improves the convergence. This development enhances the scalability of the BDDC/NNC solver in handling stochastic PDEs in three dimensions.

- In the context of DD-based intrusive SSFEM solvers for the cases involving a large number of random variables, an efficient subdomain level stochastic block matrix assembly procedure 
is developed. The new assembly routine optimizes the memory management via the recursive construction of the subdomain level sparse blocks of the stochastic finite element matrices using the subdomain level blocks of deterministic finite element matrices. Using this stochastic assembly procedure, the DD-based solvers are coupled with the FEniCS general purpose deterministic finite element package $[32,177]$. This coupling enables the DD-based solver for intrusive SSFEM to tackle a variety of PDEs implemented by using FEniCS. Therefore, it reduces the computational complexity in the code development process for the DD-based intrusive SSFEM [9, 106, 108].

- Scalable and efficient implementation of the proposed solvers is achieved with respect to stochastic dimensions by incorporating the following features. First, the solvers utilize numerous sparse data-structures and optimized routines from PETSc for commonly occurring subdomain level algebraic operations in multi-level PCGM implementations. It minimizes the memory requirements and floating point operations which are critical for simulation of SSFEM models with large number of random variables. Second, the moments of multidimensional polynomials which require thousands of multidimensional inner products are obtained using routines developed by adapting procedures from UQ Toolkit [29] involving strictly one-dimensional moments [9]. Third, the computations of large sets of multidimensional PCE/KLE basis functions and the calculation of input PCE/KLE coefficients are automated using the routines developed by adapting functions from UQ Toolkit [29].

- The performance comparison of intrusive SSFEM against non-intrusive SSFEM is presented for non-Gaussian random process modeling system parameters with increasing number of random variables. This was lacking in the numerous assessments performed in the past [101, 113, 118], which dealt with the uniform or Gaussian random variables as inputs. A comprehensive error analysis of the individual PCE coefficients of the solution process is conducted to get further insights into the convergence behavior of both intrusive and non-intrusive approaches. Furthermore, using the DD-based solver for intrusive SSFEM, efficiently imple- 
mented using advance HPC libraries, is shown to outperform the commonly used sparse grid based non-intrusive SSFEM for high-dimensional stochastic PDEs. Therefore, it emphasizes the significance of the scalable parallel algorithms and their efficient HPC implementation for uncertainty propagation in large-scale engineering systems.

- This thesis elaborates on the HPC implementational features of the solvers which were lacking in the previous articles related to these methods $[17,18]$. The algorithmic details and code snippets are presented for completeness in order to assist computational scientists in implementing these methods for their applications [19]. The detailed performance analysis and scalability studies are performed on the large-scale HPC clusters with up to 4000 cores. As a result, these developments will pave the way for the new researchers in this field to apply these solvers to various other engineering applications. To the best knowledge of the author, these algorithms have not been applied for the cases of large number of RVs and never been tested on a large-scale computing cluster at the level which is achieved in this work.

- From an application perspective, the DDM solvers are coupled with the general purpose FEM package to tackle the two- and three-dimensional steady-state diffusion equation and linear elasticity problem with the spatially varying non-Gaussian material properties. The numerical and parallel scalabilities of the solvers are presented with respect to increasing spatial and stochastic dimensions on a HPC cluster. The FEniCS based scalable DDM solvers with capabilities to tackle several stochastic PDEs are coupled with an in-house Bayesian estimation package for large-scale data assimilation problems [21,178]. The DDM solvers are used as the forecast (prediction) step to propagate the PCE coefficients of the state efficiently [21, 178]. When sensor data is available, the PCE coefficients are updated using a parallel update (analysis) step [21, 178] of the polynomial chaos Kalman filter [20,21, 178].Furthermore, the DDbased solvers for SSFEM can be used for global sensitivity analysis, i.e. the computed solution coefficients can be employed for quantifying the respective effects of input random parameters onto the variance of the response [22]. 


\subsection{Thesis Organization}

The thesis is organized in the following manner:

- Chapter 1 provides introduction to the thesis. It comprises background of the research and a brief overview of SSFEM, DDM and HPC. This is followed by a brief literature review on the solution methods utilized for the solution of intrusive SSFEM system in Sec. 1.5. Finally the problem statement, research objectives and the summary of contributions are discussed in Sec. 1.6, Sec. 1.7 and Sec. 1.8, respectively.

- Chapter 2 present the formulation of intrusive and non-intrusive spectral stochastic finite element methods for uncertainty propagation. It includes the following. In Sec. 2.1, spectral representation of stochastic processes using KLE and PCE are discussed. Next, in Sec. 2.2 the formulation of intrusive and non-intrusive spectral stochastic finite element methods are presented. This is followed by the performance comparison of intrusive and non-intrusive SSFEM approaches for stochastic PDE with a large-number of random variables in Sec. 2.3.

- Chapter 3 incorporates some of the material borrowed from the published article by the author [19]. This chapter is dedicated to the domain decomposition of stochastic PDEs in two dimensions. In Sec. 3.1, formulation and algorithms for the primal (BDDC/NNC) and dualprimal (FETI-DP) non-overlapping domain decomposition solvers for stochastic PDEs are presented. It also cover the formulation and algorithms of the one-level and two-level preconditioners. In Sec. 3.2, parallel implementation of the primal and dual-primal non-overlapping DD algorithms using two-level NNC preconditioners are given. This is followed by the parallel and numerical scalability plots for the BDDC/NNC and FETI-DP solvers from the numerical experiments performed on two-dimensional diffusion equation with stochastic diffusion coefficient modeled as a non-Gaussian stochastic process, in Sec. 3.3. Towards the end of that section, we demonstrate the advantages of DD-based intrusive SSFEM over non-intrusive SSFEM for stochastic PDEs with a large-number of non-Gaussian random variables. 
- Chapter 4 is focused on domain decomposition of stochastic PDEs in three dimensions. In Sec. 4.1, a detailed formulation for extended Schur complement of coupled stochastic PDE system is presented. Two-Level preconditioner using extended wirebasket-based coarse grid is devised in Sec. 4.2, for stochastic PDEs in three dimensions. This is followed by the numerical and parallel scalability plots of probabilistic wirebasket-based BDDC/NNC solver from the numerical simulation performed on (a) three-dimensional Poisson problem in Sec. 4.3 and (b) three-dimensional linear elasticity problem in Sec. 4.4. This chapter also include the plots for performance comparison of probabilistic wirebasket-based coarse grid against the probabilistic vertex-based coarse grid for both Poisson problem and linear elasticity problem.

- Finally, in Chapter 5, we summarize the research findings and present the directions for the future research. Sec. 5.1 briefly outlines the list of contributions and research outcomes. In Sec. 5.2 we discuss the potential limitations of the current study. This is followed by the possible extensions of this work to overcome the listed limitations and further improve the performance of the presented solvers, in Sec. 5.3. Lastly, in Sec. 5.4 we conclude the thesis. Some relevant implementational details along with the code snippets for KLE, PCE, SSFEM and DDM are presented in the Appendices. 


\section{Chapter 2}

\section{Spectral Stochastic Finite Element}

\section{Methods for Stochastic PDEs: Intrusive}

\section{Versus Non-Intrusive Approach}

The spectral stochastic finite element method (SSFEM) is a powerful numerical tool employed for UQ of stochastic PDEs [1,9]. The SSFEM is based on polynomial chaos expansion (PCE), i.e., a series representation of random vectors in terms of orthogonal polynomials $[1,9,12]$. Here the term spectral is used because of the representation of a stochastic process as an infinite linear combination of orthogonal functions and their coefficients, which is analogous to a Fourier series representation of a function and it involves eigenvalue analysis of covariance function. Similar to the spectral method used in the numerical solution of deterministic differential equations [179], the SSFEM is a global approach, i.e. the selected basis functions are nonzero over the whole domain [1]. The application of SSFEM requires: (a) spatial discretization of a stochastic PDE using a FEM, (b) stochastic discretization of the random system parameters, stochastic source term and solution process using the PCE, followed by a standard Galerkin projection along the random dimensions, and (c) the resulting system is solved for the PCE coefficients of the solution process using an intrusive or non-intrusive approach $[1,2,9,10]$. 
This chapter is organized in the following manner. Sec. 2.1 is dedicated for the spectral representation of input and output stochastic processes using the Karhunen-Loève expansion (KLE) and polynomial chaos expansion (PCE). This is followed by the formulation and implementation of the intrusive and non-intrusive SSFEM in the Sec. 2.2. Finally, in Sec. 2.3, we compare the performance of intrusive and non-intrusive approaches for non-Gaussian random system parameters in terms of error analysis in the solution coefficients.

\subsection{Spectral Representation of Stochastic Processes}

In numerous engineering applications, the system parameters and/or the initial and boundary conditions vary randomly across the physical domain of the problem. If sufficient statistical information is available, uncertainty in the system parameters can be modeled as random vectors or random processes characterized by multiple random variables $[1,9]$. Representing uncertainty as a random vector necessitates its joint probability density function (PDF). On the other hand, the complete representation of uncertainty in the form of stochastic process requires $n$-th order (indexed by space or time or both) joint PDF of the process $[1,2,9,180]$. Usually it is not possible to estimate the joint PDF of any arbitrary order of a random process in practical engineering applications. Therefore, the representation of an non-stationary and non-Gaussian stochastic process is often restricted to marginal PDF and the auto-correlation function $[1,180,181]$.

The most widely utilized approaches for the spectral representation of stochastic processes are the KLE and PCE $[1,9,71]$. These methods are based on expanding the random function in a Fourier-type series [1,9]. In the following sections, the mathematical details on the KLE and PCE representation of stochastic process are provided. In Sec. 2.1.1 and 2.1.2 we present the formulation of the KLE and PCE, respectively. Note that, in this chapter, we will largely focus on the implementation aspects of these approaches. The theoretical details of the KLE and PCE are available in the numerous articles $[1,2,9,61,64,71,76,182,183]$. 


\subsubsection{Karhunen-Loève Expansion}

The Karhunen-Loève expansion (KLE) is an useful tool available to characterize the random process with a set of random variables. It is based on the spectral decomposition of covariance kernel to obtain the set of eigenvalues and eigenfunctions used to characterize the stochastic process $[1,2,9]$.

Consider $\alpha(\boldsymbol{x}, \boldsymbol{\xi}(\theta))$ to be a stochastic process, a function of the position vector $\boldsymbol{x}$ defined over physical domain $\mathscr{D}$ and the set of random variables $\boldsymbol{\xi}=\left\{\xi_{n}\right\}_{n=1}^{\infty}$ which are functions of random event $\theta$ defined by the complete probability space $(\Omega, \mathscr{E}, \mathscr{P})$. Here $\Omega$ is a sample space, $\mathscr{E}$ is a set of events and $\mathscr{P}$ is a probability measure (refer [181] for more details). The KL expansion of such a stochastic process can be written as [1,2],

$$
\alpha(\boldsymbol{x}, \boldsymbol{\xi}(\theta))=\bar{\alpha}(\boldsymbol{x})+\sum_{n=1}^{\infty} \sqrt{\lambda_{n}} f_{n}(\boldsymbol{x}) \xi_{n}(\theta)
$$

where $\bar{\alpha}(\boldsymbol{x})$ is the expected value of the random process and $\left\{\xi_{n}\right\}$ is a set of uncorrelated (not necessarily independent) random variables. For a Gaussian stochastic process, $\left\{\xi_{n}\right\}_{n=1}^{\infty}$ is a set of independent Gaussian random variables. The eigenvalues $\left\{\lambda_{n}\right\}_{n=1}^{\infty}$ and eigenfunctions $\left\{f_{n}\right\}_{n=1}^{\infty}$ of the covariance function $C_{\alpha \alpha}\left(\boldsymbol{x}_{1}, \boldsymbol{x}_{2}\right)$ are obtained by solving the following integral equation [1]

$$
\int_{\Omega} C_{\alpha \alpha}\left(\boldsymbol{x}_{1}, \boldsymbol{x}_{2}\right) f_{n}\left(\boldsymbol{x}_{1}\right) d \boldsymbol{x}_{1}=\lambda_{n} f_{n}\left(\boldsymbol{x}_{2}\right)
$$

Although an infinite number of KLE terms are showed in Eq. (2.1), for numerical simulation, a finite number of KLE modes $(L)$ are sufficient for a smooth stochastic process [1,9]. The truncated representation of stochastic process is represented as,

$$
\alpha(\boldsymbol{x}, \boldsymbol{\xi}(\theta)) \approx \bar{\alpha}(\boldsymbol{x})+\sum_{n=1}^{L} \sqrt{\lambda_{n}} f_{n}(\boldsymbol{x}) \xi_{n}(\theta) .
$$

The number of KLE modes used in Eq. (2.3) is equal to the number of random variables required to approximate the stochastic process $[1,2]$. 
The selection of the number of KLE modes is based on the correlation length of the underlying stochastic process. A strongly correlated (i.e., narrow bandwidth) process with a long correlation length needs fewer expansion terms to capture the uncertainty. Conversely, if the correlation length is short, i.e., a weakly correlated (broad bandwidth) process demands more expansion terms [1,9,64]. Fig. 2.1 plots the relative partial sum of the eigenvalue contribution for the exponential covariance kernel given in Eq. (2.4). For the correlation length, $b=1$ we need only 12 modes to capture $95 \%$ of relative contribution as oppose to 35 modes when the correlation length reduced to $b=0.3$. Note that the number of random variables (i.e., the number of KLE modes) also refers to the dimension of the stochastic process in this thesis.

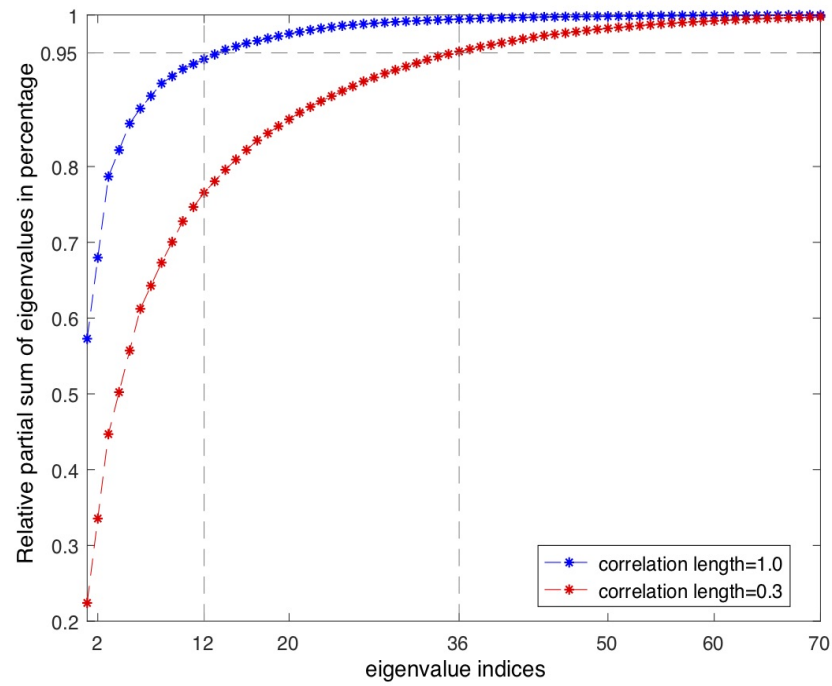

Figure 2.1: Relative partial sum of eigenvalues for exponential covariance kernel on a unit square domain.

Following the book by Ghanem and Spanos [1], a semi-analytical expression of the KLE is detailed for a Gaussian process. Consider an exponential covariance function for a Gaussian stochastic process defined over a two-dimensional physical domain $\mathscr{D}(x, y)$ over the interval $\left[\begin{array}{ll}-a & a\end{array}\right] \times\left[\begin{array}{ll}-a & a\end{array}\right][1]$

$$
C_{\alpha \alpha}\left(x_{1}, y_{1} ; x_{2}, y_{2}\right)=\sigma^{2} e^{-\left|x_{2}-x_{1}\right| / b_{1}-\left|y_{2}-y_{1}\right| / b_{2}}
$$


where $b_{1}$ and $b_{2}$ are the correlation lengths along $x$ and $y$ directions respectively and $\sigma^{2}$ denotes the variance of the underlying stochastic process.

Solving the integral given in Eq. (2.2) for the covariance kernel given in Eq. (2.4), the eigenvalues and eigenfunctions are obtained as [1],

$$
\begin{gathered}
\lambda_{n}=\lambda_{i}^{x} \otimes \lambda_{i}^{y}, \\
f_{n}(x, y)=g_{i}(x) \otimes h_{i}(y) .
\end{gathered}
$$

where $\otimes$ denotes the tensor product (for example, see Table A.1 in Appendix A.1).

The two-dimensional eigenfunctions $\left\{f_{n}\right\}_{n=1}^{\infty}$ and eigenvalues $\left\{\lambda_{n}\right\}_{n=1}^{\infty}$ are computed as the tensor product of the one-dimensional eigenfunctions $\left\{g_{i}(x), h_{i}(y)\right\}$ and eigenvalues $\left\{\lambda_{i}^{x}, \lambda_{i}^{y}\right\}$ respectively $[1,182]$. The analytical expressions for the one-dimensional eigenvalues and eigenfunctions for the exponential covariance kernel in Eq. (2.4) are [1,182],

$$
\begin{gathered}
\lambda_{i}^{x}=\sigma \frac{2 b_{1}}{1+b_{1}^{2} \omega_{i}^{2}}, \\
\lambda_{i}^{y}=\sigma \frac{2 b_{2}}{1+b_{2}^{2} \omega_{i}^{2}}, \\
g_{i}(\boldsymbol{x})=h_{i}(\boldsymbol{x})=\left\{\begin{array}{cl}
\frac{\cos \left(\omega_{i} \boldsymbol{x}\right)}{\sqrt{a+\frac{\sin \left(2 \omega_{i} a\right)}{2 \omega_{i}}}}, & \text { for } i \text { odd }, \\
\frac{\sin \left(\omega_{i} x\right)}{\sqrt{a-\frac{\sin \left(2 \omega_{i} a\right)}{2 \omega_{i}}}}, & \text { for } i \text { even },
\end{array}\right.
\end{gathered}
$$

where $\omega_{i}$ 's are the solution of the following transcendental equations given by $[1,182]$

$$
\begin{aligned}
& \frac{1}{b}-\omega_{i} \tan \left(\omega_{i} a\right)=0, \text { for } i \text { odd } \\
& \omega_{i}+\frac{1}{b} \tan \left(\omega_{i} a\right)=0, \text { for } i \text { even. }
\end{aligned}
$$

The numerical implementation of KLE can be summarized in the following steps.

- Solve the transcendental equations given in Eq. (2.9) for $\omega_{i}$ 's. 
- Substitute $\omega_{i}$ 's in Eq. (2.7) and (2.8) to compute the one-dimensional eigenvalues and eigenfunctions respectively.

- Using Eq. (2.5) and (2.6) compute the two-dimensional eigenvalues $\left\{\lambda_{n}\right\}_{n=1}^{\infty}$ and eigenfunction $\left\{f_{n}\right\}_{n=1}^{\infty}$ respectively and

- using $\lambda_{n}$ and $f_{n}$ in Eq. (2.3), compute the KLE modes.

Refer to Appendix A and B for the further details on the implementation of KLE with the code snippets. Note that the procedure presented above is for a two-dimensional physical domain. However, a similar procedure can be used for the cases with three-dimensional domains.

\subsubsection{Polynomial Chaos Expansion}

The KLE is a powerful tool that can be exploited in characterizing a stochastic process with a known covariance function. However, the KLE cannot be used to represent a process with unknown covariance function. In such cases, the PCE can be utilized [1,9].

A second order stochastic process $\alpha(\boldsymbol{x}, \boldsymbol{\xi}(\theta))$, i.e., a random process with finite variance can be expanded using PCE as [1],

$$
\alpha(\boldsymbol{x}, \boldsymbol{\xi}(\theta))=\sum_{j=0}^{\infty} \widehat{\alpha}_{j}(\boldsymbol{x}) \Psi_{j}(\boldsymbol{\xi}(\theta)),
$$

where $\Psi_{j}(\boldsymbol{\xi}(\theta))$ is a set of multidimensional orthogonal polynomials of the order $p_{\alpha}$ of $L$ independent random variables $\boldsymbol{\xi}=\left\{\xi_{1}, \xi_{2}, \ldots, \xi_{L}\right\}$ and $\widehat{\alpha}_{j}(\boldsymbol{x})$ represents the unknown deterministic coefficients. Although the PCE is mainly founded on the independence assumption of the random variables, for some applications, there may exist significant dependence among the random variables. In such cases the Rosenblatt transformation [184], commonly employed for mapping dependent to independent variables can be exploited (see the following articles for further details $[185,186])$. 
The polynomials $\Psi_{j}(\boldsymbol{\xi})$ (note that the argument $\theta$ of $\boldsymbol{\xi}$ being dropped hereafter for notational simplicity) are orthogonal in a statistical sense, i.e., their inner product with respect to probability measure is zero. Therefore, $\left\langle\Psi_{i}(\boldsymbol{\xi}), \Psi_{j}(\boldsymbol{\xi})\right\rangle=0$ for $i \neq j$ and for $i=j$, it can be computed as the statistical average as shown below [1],

$$
\left\langle\Psi_{i}(\boldsymbol{\xi}), \Psi_{j}(\boldsymbol{\xi})\right\rangle=\int \Psi_{i}(\boldsymbol{\xi}) \Psi_{j}(\boldsymbol{\xi}) \mathrm{p}(\boldsymbol{\xi}) d \boldsymbol{\xi}
$$

where $\mathrm{p}($.$) is the joint probability density function (PDF) of \boldsymbol{\xi}$, obtained from the marginal PDF of $\mathrm{p}_{\mathrm{i}}\left(\boldsymbol{\xi}_{\mathrm{i}}\right)$ as: $\mathrm{p}(\boldsymbol{\xi})=\prod_{i=1}^{L} \mathrm{p}_{\mathrm{i}}\left(\xi_{\mathrm{i}}\right)$.

Theoretically, we need an infinite number of PCE terms to accurately approximate the output process. However, for the numerical approximation, the truncated PCE with $P_{\alpha}$ expansion terms can be used to obtain a sufficiently accurate representation of the output. Note that the higher order moments in the probability density function can be the measure of accuracy. The truncated expansion given in Eq. (2.12) is shown to converge in the mean square sense [1]:

$$
\alpha(\boldsymbol{x}, \boldsymbol{\xi}) \approx \sum_{j=0}^{P_{\alpha}} \widehat{\alpha}_{j}(\boldsymbol{x}) \Psi_{j}(\boldsymbol{\xi})
$$

where the $P_{\alpha}$ is a function of the order of polynomials $p_{\alpha}$ and the number of random variables $L$ as given below [1],

$$
P_{\alpha}=\frac{\left(L+p_{\alpha}\right) !}{L ! p_{\alpha} !}-1
$$

The high-frequency random fluctuations in the stochastic process demand a large number of random variables and the strong nonlinear dependences of the solution process on the random system parameters necessitate the higher-order polynomials $p_{\alpha}$ in the PCE representation $[2,182,187]$. Note that the order of polynomials and the number of random variables can differ in the input and output PC expansions based on the requirement. In general, for better convergence, the output processes are represented using higher order expansions than the input random processes $[2,9]$. This is because the uncertainty in the output process may amplify 
as the input uncertainty undergoes nonlinear transformation through the model. This demands higher order expansion terms to capture the nonlinear and non-Gaussian effects. For further details on the convergence studies refer to the following articles $[2,182,187]$. The implementational details and the associated code snippets of the PCE for a non-Gaussian stochastic process are presented in Appendices A and B.

\subsection{Spectral Stochastic Finite Element Methods}

In this section, we briefly describe the formulations of the intrusive and non-intrusive spectral stochastic finite element methods. For an elementary exposition of both the methodologies, we consider a linear elliptic stochastic PDE on a spatial domain $\mathscr{D}$ with the known boundary condition on $\partial \mathscr{D}$. Consider a two-dimensional steady-state flow through random media with a spatially varying non-Gaussian diffusion coefficient $c_{d}$. The flow is modeled by a twodimensional stochastic diffusion equation. This leads to a Poisson problem defined by a linear elliptic stochastic PDE as defined below:

$$
\begin{aligned}
-\nabla \cdot\left(c_{d}(\boldsymbol{x}, \theta) \nabla u(\boldsymbol{x}, \theta)\right) & =F(\boldsymbol{x}), & \mathscr{D} \times \Omega, \\
u(\boldsymbol{x}, \theta) & =0, & \partial \mathscr{D} \times \Omega,
\end{aligned}
$$

where $\nabla$ denotes the gradient which represents the differential operator with respect to the spatial variables $\boldsymbol{x}, u$ is the solution process, $\theta$ is an element in the sample space $\Omega$ defined by the probability space $(\Omega, \mathscr{F}, \mathscr{P})[181]$. For the sake of convenience, $F(\boldsymbol{x})$ is modeled as a deterministic source term. However, the methodology presented herein can be easily extended to stochastic source function $F(\boldsymbol{x}, \theta)$.

To solve a partial differential equation, for instance Eq. (2.14), using finite element methods the PDE needs to be expressed in the variational form $[9,73]$. To do so, first, the Eq. (2.14) is 
multiplied by a test function $v$ and performing integration by parts we obtain [73]:

$$
\int_{\mathscr{D}} c_{d}(\boldsymbol{x}, \theta) \nabla u \cdot \nabla v d \boldsymbol{x}=\int_{\mathscr{D}} F v d \boldsymbol{x}+\int_{\partial \mathscr{D}} \frac{\partial u}{\partial n} v d s
$$

where $u$ is called the trial function, $v$ is called the test function and $\partial u / \partial n$ is the derivative of $u$ in the outward normal direction at the boundary. Note that $v$ vanishes where $u$ is known, i.e., on $\partial \mathscr{D}$. Therefore, the resulting variation form can be further simplified as:

$$
\int_{\mathscr{D}} c_{d}(\boldsymbol{x}, \theta) \nabla u \cdot \nabla v d \boldsymbol{x}=\int_{\mathscr{D}} F v d \boldsymbol{x} .
$$

Consider the diffusion coefficient $c_{d}(\boldsymbol{x}, \theta)$, being modeled as a lognormal stochastic process $l(\boldsymbol{x}, \theta)$ obtained by an exponential of a Gaussian process $g(\boldsymbol{x}, \theta)$ having the mean $g_{0}(\boldsymbol{x})$, variance $\sigma^{2}$ and exponential covariance function $C_{g g}(x, y)$ in Eq. (2.4). For illustration, $c_{d}(\boldsymbol{x}, \theta)$ is characterized by using two random variables with second order PCE. Therefore, PCE of the lognormal stochastic process can be expressed as [1] (see Appendix A.3 for details).

$$
\begin{aligned}
c_{d}(\boldsymbol{x}, \theta) & =l_{0}(\boldsymbol{x})\left(g_{0}+\xi_{1}(\theta) g_{1}(\boldsymbol{x})+\xi_{2}(\theta) g_{2}(\boldsymbol{x})+\left(\xi_{1}^{2}(\theta)-1\right) \frac{g_{1}^{2}(\boldsymbol{x})}{2}\right. \\
& \left.+\left(\xi_{1}(\theta) \xi_{2}(\theta)\right) g_{1}(\boldsymbol{x}) g_{2}(\boldsymbol{x})+\left(\xi_{2}^{2}(\theta)-1\right) \frac{g_{2}^{2}(\boldsymbol{x})}{2}\right),
\end{aligned}
$$

where $\left\{g_{i}(\boldsymbol{x})\right\}_{i=1}^{2}$ are the scaled eigenfunctions of the covariance kernel $C_{g g}(x, y)$ and $l_{0}(\boldsymbol{x})=$ $\exp \left[g_{0}(\boldsymbol{x})+\frac{1}{2} g_{1}^{2}(\boldsymbol{x})+\frac{1}{2} g_{2}^{2}(\boldsymbol{x})\right]$ is the mean of the lognormal process.

Consequently, Eq. (2.17) can be re-written as

$$
\begin{aligned}
& \int_{\mathscr{D}} l_{0}(\boldsymbol{x})\left(g_{0}+\xi_{1}(\theta) g_{1}(\boldsymbol{x})+\xi_{2}(\theta) g_{2}(\boldsymbol{x})+\left(\xi_{1}^{2}(\theta)-1\right) \frac{g_{1}^{2}(\boldsymbol{x})}{2}\right. \\
& \left.+\left(\xi_{1}(\theta) \xi_{2}(\theta)\right) g_{1}(\boldsymbol{x}) g_{2}(\boldsymbol{x})+\left(\xi_{2}^{2}(\theta)-1\right) \frac{g_{2}^{2}(\boldsymbol{x})}{2}\right) \nabla u . \nabla v d \boldsymbol{x}=\int_{\mathscr{D}} F v d \boldsymbol{x} .
\end{aligned}
$$


The finite element discretization with $N$ nodes in the spatial domain leads to a system of linear equations with random coefficients $\theta$ denotes stochasticity [1]

$$
\mathbf{A}(\theta) \mathbf{u}(\theta)=\mathbf{f}
$$

where $\mathbf{A}(\theta)$ is the random or stochastic system matrix, $\mathbf{u}(\theta)$ is the stochastic response vector and f is the deterministic source vector. In the deterministic setting the above system of equations can be solved for the mean values of the stochastic parameters. For example, the Gaussian process characterized by using $g_{0}=0, \sigma=0.3$ and the exponential covariance function given in Eq. (2.4) with $b_{1}=b_{2}=b=1$. Therefore, $c_{d}$ can be expanded as [1] (for simplification using $L \rightarrow \infty$, refer to Appendix A.3 for details).

$$
c_{d}(\boldsymbol{x}, \theta)=l_{0}=\exp \left[0+\frac{1}{2} 0.3^{2}\right]=1.05
$$

Consider a unit square domain discretized using unstructured finite element mesh with 600 nodes and 1200 elements as shown in Fig. 2.2. The numerical simulations are performed using the unit source term. The solution field for the mean value of stochastic parameter is shown in Fig. 2.2. The corresponding FEniCS based python code snippet is shown in Listing B.1.
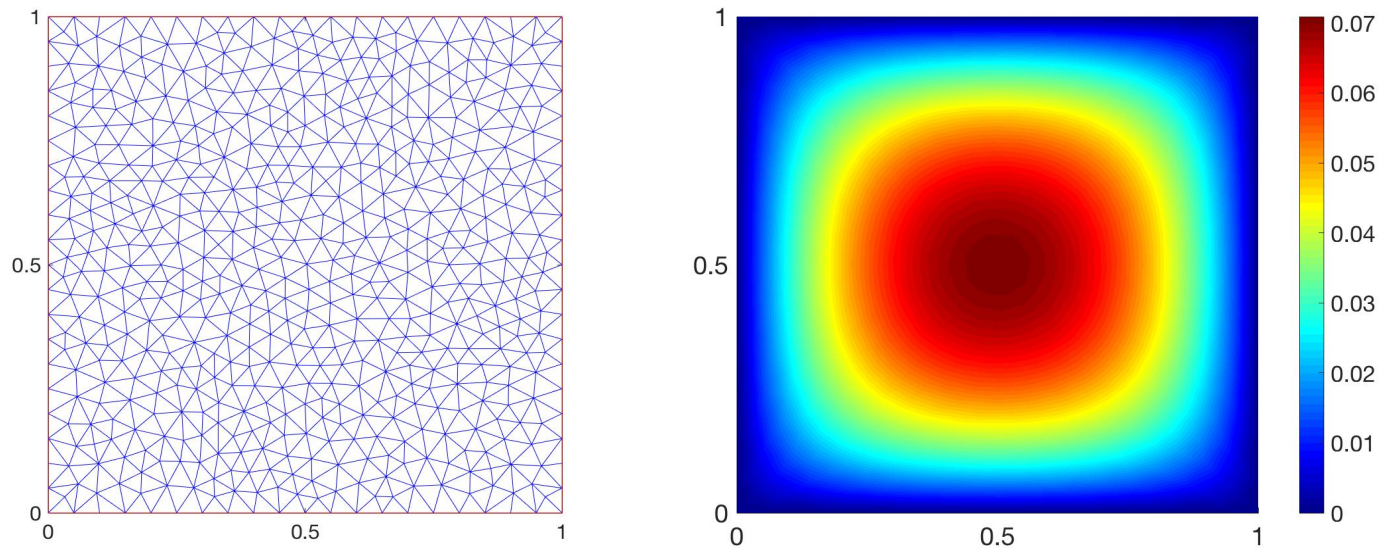

Figure 2.2: Finite element mesh and the solution field at the mean value of stochastic system parameters. 
Assuming that the input data contains sufficient statistical information, we can approximate the random system matrix and the stochastic solution process using the PCE as [1]:

$$
\mathbf{A}(\theta) \approx \sum_{i=0}^{P_{\mathrm{A}}} \hat{\mathbf{A}}_{i} \Psi_{i}(\boldsymbol{\xi}), \quad \mathbf{u}(\theta) \approx \sum_{j=0}^{P_{u}} \hat{\mathbf{u}}_{j} \Psi_{j}(\boldsymbol{\xi})
$$

where the $\hat{\mathbf{A}}_{i}$ 's are the PCE coefficients of the random system matrix, $\hat{\mathbf{u}}_{j}$ 's are the PCE coefficients of the solution process and $\Psi$ 's are the multidimensional polynomials obtained as a function of $L$ random variables $\boldsymbol{\xi}=\left\{\xi_{1}, \xi_{2}, \ldots, \xi_{L}\right\} . P_{\mathrm{A}}$ and $P_{u}$ are the numbers of PCE terms required to characterize the stochastic system matrix and the stochastic response vector respectively [1]. The number of PCE terms $P_{\alpha}$ is a function of the order of polynomials $p_{\alpha}$ and the number of random variables $L$, which can be computed using Eq. (2.13) [1] and the subscript $\alpha$ represents A or $u$. In this study the input, namely the diffusion coefficient of the Poisson PDE is modeled by a lognormal process obtained by the exponential of a Gaussian process.

Appendix A details the PCE of the lognormal process and KLE of the underlying Gaussian process. In the SSFEM approaches, the primary goal is to compute the PCE coefficients of the solution process $\hat{\mathbf{u}}_{j}$. Based on the approach utilized to compute $\hat{\mathbf{u}}_{j}$, the solution methodologies for SSFEM are generally classified into (a) intrusive (sampling-free) approach also referred to as the stochastic Galerkin (SG) approach [1,10,23,90,143] and (b) non-intrusive (sampling-based) approaches such as non-intrusive spectral projection (NISP) $[9,79,101,106,108]$ or stochastic collocation (SC) approach $[62,80,110,111]$. Note that, in this thesis, we have focused on the SG and the NISP approach based on the sparse grid quadrature.

In the SG (intrusive) approach, the PCE for the random system parameters and the solution process, for example as given in Eq. (2.22) are substituted into the governing equation (for example Eq. (2.20)). In SG framework the strategy is to project the residual onto a finitedimensional space spanned by appropriate orthogonal basis functions with respect to probability 
density $\mathrm{p}(\boldsymbol{\xi})$. Thus we seek the approximate solution as [12]

$$
\begin{aligned}
0 & =\left\langle\mathbf{A}(\theta) \mathbf{u}(\theta)-\mathbf{f}, \Psi_{k}(\boldsymbol{\xi})\right\rangle, k=0,1, \ldots, P_{u}, \\
& =\int_{\mathcal{D}}\left[\sum_{i=0}^{P_{\mathrm{A}}} \hat{\mathbf{A}}_{i} \Psi_{i}(\boldsymbol{\xi}) \sum_{j=0}^{P_{u}} \hat{\mathbf{u}}_{j} \Psi_{j}(\boldsymbol{\xi})-\mathbf{f}\right] \Psi_{k}(\boldsymbol{\xi}) \mathrm{p}(\boldsymbol{\xi}) d \boldsymbol{\xi}, k=0,1, \ldots, P_{u} .
\end{aligned}
$$

Eq. (2.23) can also be approximately solved using the quadrature rule with quadrature points $q_{n}$ and weights $w_{n}$ as

$$
\sum_{n=1}^{N}\left[\sum_{i=0}^{P_{\mathrm{A}}} \hat{\mathbf{A}}_{i} \Psi_{i}\left(q_{n}\right) \sum_{j=0}^{P_{u}} \hat{\mathbf{u}}_{j} \Psi_{j}\left(q_{n}\right)-\mathbf{f}\right] \Psi_{k}\left(q_{n}\right) \mathrm{p}\left(q_{n}\right) w_{n}=0, \quad k=0,1, \ldots, P_{u} .
$$

In the NISP framework (also referred as pseudo-spectral approach) the PCE coefficients of solution process are obtained by performing Galerkin projection on the PCE of the solution process given in Eq. (2.22). The orthogonality properties of PCE basis functions are utilized to compute the expansion coefficients of the solution process [1,9] as,

$$
\hat{\mathbf{u}}_{k}=\frac{1}{\left\langle\Psi_{k}^{2}(\boldsymbol{\xi})\right\rangle} \sum_{n=1}^{N} \mathbf{u}\left(q_{n}\right) \Psi_{k}\left(q_{n}\right) \mathrm{p}\left(q_{n}\right) w_{n}, \quad k=0,1, \ldots, P_{u}
$$

The NISP approach is simple to implement; however, for the cases with a large number of random variables (which is the primary focus of this thesis) the number of quadrature points required to solve the above integral increases significantly [12,79, 106, 162].

In the framework of collocation approach, $M$ collocation points are generated from the parameter space, and then enforce

$$
\mathbf{u}\left(q_{m}\right)=\tilde{\mathbf{u}}\left(q_{m}\right), \quad m=1, \ldots, M
$$

to compute $\hat{\mathbf{u}}_{j}$. For a general basis function $\phi_{k}$, performing above steps yields the following system [12] 


$$
\left[\begin{array}{ccc}
\phi_{0}\left(q_{1}\right) & \ldots & \phi_{P_{u}}\left(q_{1}\right) \\
\vdots & \ddots & \vdots \\
\phi_{0}\left(q_{M}\right) & \ldots & \phi_{P_{u}}\left(q_{M}\right)
\end{array}\right]\left\{\begin{array}{c}
\hat{\mathbf{u}}_{0} \\
\vdots \\
\hat{\mathbf{u}}_{P_{u}}
\end{array}\right\}=\left\{\begin{array}{c}
\mathbf{u}\left(q_{1}\right) \\
\vdots \\
\mathbf{u}\left(q_{M}\right)
\end{array}\right\}
$$

Similar to the NISP approach, the SC approach is also straightforward to formulate. However, for a large number of random variables, the number and choice of collocation points as well as the choice of basis function become critical [12]. Note that the computational efforts required for the collocation approach is essentially equivalent to that of NISP approach when the number of collocation points $M$ equal to quadrature points $N$.

The accuracy of intrusive (SG) approach is optimal in $\mathrm{L}^{2}$ sense. Therefore, the $\mathrm{SG}$ approach can be computationally efficient over NISP or SC methods [12]. However, SG approach involves substantial modification of a deterministic simulation code [12]. Note that, later in this chapter, we demonstrate that the requirement of modification of a deterministic simulation code due to intrusive adjustments in the SG approach can be alleviated by employing general purpose deterministic FEM packages (e.g. FEniCS [177]).

On the other hand, the SC approach does not encounter orthogonality and dependence requirement issues observed in SG approach. The SC approach can be applied to general parameter distribution (see $[12,106]$ for more details). However, the SC is a sampling-based approach and the number of required samples grow with the order and the number of random variables. Similarly, in the NISP approach the number of quadrature points grow with the order and number of random variables. Note that, the SC approach is not implemented in this thesis, instead we use NISP approach based on the sparse grid quadrature. For more details on the SC approach and its comparison with NISP and SG approaches the author refer to the numerous articles available in the literature [12,62,80,106,110,111].

The rest of this chapter is organized as follows: Sec. 2.2.1 outlines the formulation of intrusive SSFEM (SG). This is followed by the formulation of non-intrusive approach, specifically, 
the NISP with Smolyak sparse grid quadrature in Sec. 2.2.2. In Sec. 2.3 the performance comparison of the intrusive SSFEM with the non-intrusive SSFEM is conducted using a Poisson equation having a non-Gaussian diffusion coefficient, modeled by a lognormal process.

\subsubsection{Intrusive SSFEM}

In the intrusive SSFEM, the PCE of the system matrix with random coefficients $\mathbf{A}(\theta)$ and the solution process $\mathbf{u}(\theta)$, presented in Eq. (2.22) are directly substituted into the finite element discretization of stochastic PDE given in Eq. (2.20) leading to [1]

$$
\epsilon=\sum_{i=0}^{P_{\mathrm{A}}} \hat{\mathbf{A}}_{i} \Psi_{i}(\boldsymbol{\xi}) \sum_{j=0}^{P_{u}} \hat{\mathbf{u}}_{j} \Psi_{j}(\boldsymbol{\xi})-\mathbf{f} \neq 0
$$

where $\epsilon$ is the random residual.

Performing Galerkin projection, i.e., multiplying both sides of the Eq. (2.28) by $\Psi_{k}(\boldsymbol{\xi})$ with $k=0, . ., P_{u}$ and taking expectation both sides results in the following system of coupled equations [1],

$$
\begin{gathered}
\left\langle\epsilon, \Psi_{k}(\boldsymbol{\xi})\right\rangle=0, \quad k=0,1, \ldots, P_{u} \\
\sum_{j=0}^{P_{u}} \sum_{i=0}^{P_{\mathrm{A}}}\left\langle\Psi_{i}(\boldsymbol{\xi}) \Psi_{j}(\boldsymbol{\xi}) \Psi_{k}(\boldsymbol{\xi})\right\rangle \hat{\mathbf{A}}_{i} \hat{\mathbf{u}}_{j}=\left\langle\mathbf{f} \Psi_{k}(\boldsymbol{\xi})\right\rangle, \quad k=0,1, \ldots, P_{u} .
\end{gathered}
$$

For notational convenience, we rewrite Eq. (2.30) using $\left\langle\Psi_{i}(\boldsymbol{\xi}) \Psi_{j}(\boldsymbol{\xi}) \Psi_{k}(\boldsymbol{\xi})\right\rangle=\mathcal{C}_{i j k}$ and $\left\langle\mathbf{f} \Psi_{k}(\boldsymbol{\xi})\right\rangle=$ $f_{k}$ leading to

$$
\sum_{j=0}^{P_{u}} \sum_{i=0}^{P_{\mathrm{A}}} \mathcal{C}_{i j k} \hat{\mathbf{A}}_{i} \hat{\mathbf{u}}_{k}=f_{k}, \quad k=0,1, \ldots, P_{u} .
$$

For concise representation, the following notation is used,

$$
A_{j k}=\sum_{i=0}^{P_{\mathrm{A}}} \mathcal{C}_{i j k} \hat{\mathbf{A}}_{i}
$$


Thus, Eq. (2.31) can be further simplified as,

$$
\sum_{j=0}^{P_{u}} A_{j k} \hat{\mathbf{u}}_{k}=f_{k}, \quad k=0,1, \ldots, P_{u}
$$

Equivalently Eq. (2.33) can be written as,

$$
[\mathscr{A}]\{\mathscr{U}\}=\{\mathscr{F}\}
$$

where $\mathscr{A}$ in Eq. (2.34) is the system matrix, $\mathscr{U}$ is the vector of PCE coefficients of the solution process and $\mathscr{F}$ is the corresponding right hand side vector arising in the setting of intrusive SSFEM. Eq. (2.34) can be expanded as

$$
\left[\begin{array}{cccc}
A_{0,0} & A_{0,1} & \ldots & A_{0, P_{u}} \\
A_{1,0} & A_{1,1} & \ldots & A_{1, P_{u}} \\
\vdots & \vdots & \ddots & \vdots \\
A_{P_{u}, 0} & A_{P_{u}, 1} & \ldots & A_{P_{u}, P_{u}}
\end{array}\right]\left\{\begin{array}{c}
\hat{\mathbf{u}}_{0} \\
\hat{\mathbf{u}}_{1} \\
\vdots \\
\hat{\mathbf{u}}_{P_{u}}
\end{array}\right\}=\left\{\begin{array}{c}
f_{0} \\
f_{1} \\
\vdots \\
f_{P_{u}}
\end{array}\right\}
$$

The solution of the above coupled linear system yields the PCE coefficients of the solution process. These PCE coefficients can be used to obtain the mean, variance, higher order statistics and PDF of the solution process [1].

The size of the system matrix $\mathscr{A}$ is $\left(N \times P_{u}, N \times P_{u}\right)$, where $N$ is the number of degree-offreedom related to the finite element mesh resolution and $P_{u}$ is the number of PCE terms used in the representation of the solution process. Note that, $P_{u}$ is a function of stochastic dimension $L$ and order $p_{u}$ of the PCE. From Eq. (2.32) and (2.33) it can be noted that, each block of the system matrix $\mathscr{A}$ is denoted by $A_{j k}$ (a sub matrix of size $\left.(N \times N)\right)$ and it can be computed from the set of deterministic finite element matrices $\hat{\mathbf{A}}_{i}$. In this thesis, the system matrix assembly procedure, i.e., implementation of Eq. (2.32) is performed by employing deterministic finite element assembly routines imported from the FEniCS general purpose FEM package [32, 188]. 
The intrusive system matrix $\mathscr{A}$ has the two-levels of sparsity (block-sparse structure), for example, see in Fig. 2.3 and 2.4 [10,90, 182]. The first-level of sparsity is coming from the deterministic finite element matrices, $\hat{\mathbf{A}}_{i}$, which are sparse and the second-level of sparsity is due to the stochastic aspects of the problem arising from a few non-zero $\mathcal{C}_{i j k}$ terms. The matrices in Fig. 2.3 and 2.4 are assembled for the stochastic diffusion equation with a lognormal diffusion coefficient represented using the underlying Gaussian stochastic process (refer to Appendix B for further details).
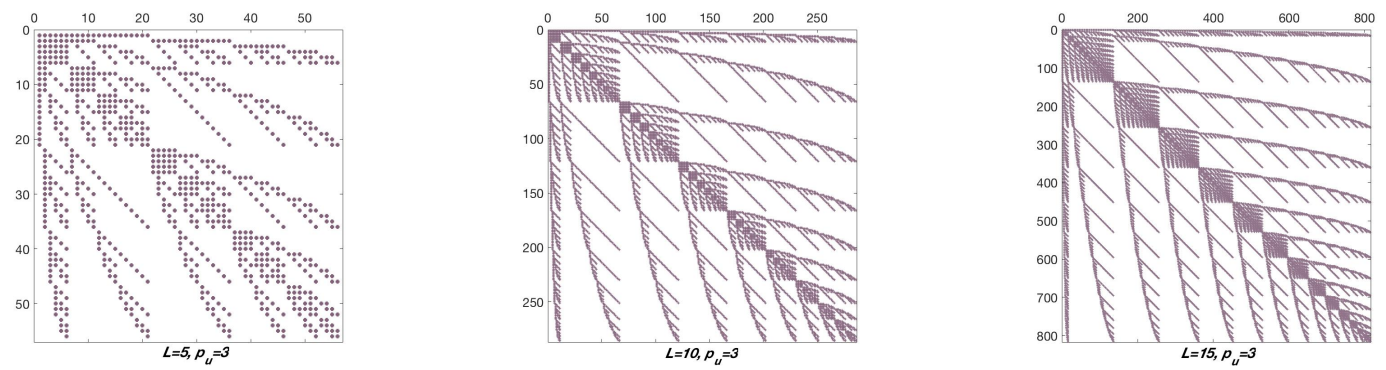

Figure 2.3: SSFEM system matrices for the fixed $N=30, p_{\mathrm{A}}=2, p_{u}=3$ with $L=5, L=10$ and $L=15$.
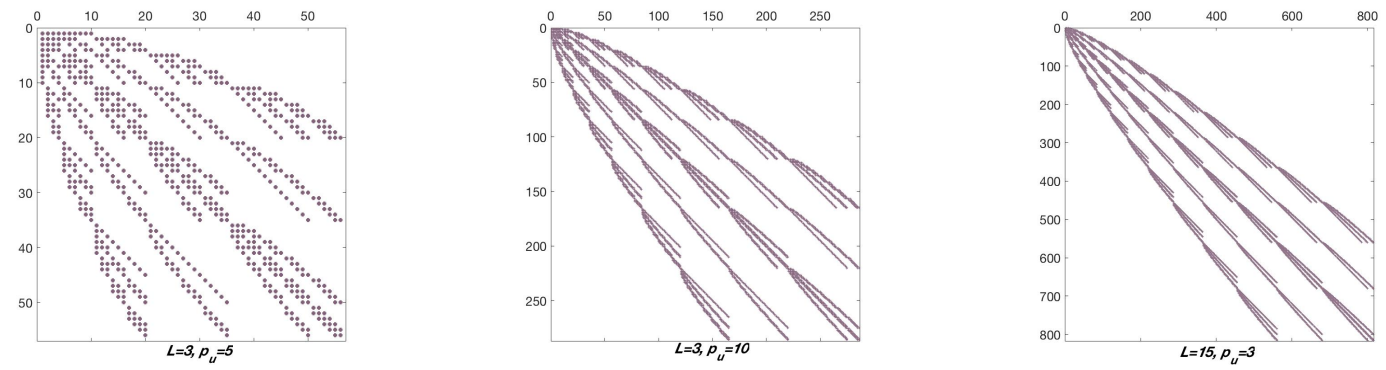

Figure 2.4: SSFEM system matrices for the fixed $N=30, p_{\mathrm{A}}=2, L=3$ with

$$
p_{u}=5, p_{u}=10 \text { and } p_{u}=15 \text {. }
$$

The sparsity structure of each block $\hat{\mathbf{A}}_{i}$ in the intrusive system matrix depends on finite element discretization. Therefore, its sparsity pattern is not influenced by stochastic expansion terms such as $L$ or $p_{u}$. However, the block-sparsity structure of the system matrix changes with (a) the number of random variables as shown in Fig. 2.3 (for fixed order of expansion and varying number of random variables) and (b) the order of expansion as shown in Fig. 2.4 (for fixed number of random variables and varying order of expansion). The orders and dimensions 
of the input and output stochastic processes influence the number of nonzero $\mathcal{C}_{i j k}$ terms, and thus the number of non-zero blocks in $\mathscr{A}$. Consequently, the size of the intrusive system matrix and vector grows rapidly with the number of random variables and order of expansion. This is the primary challenge of the intrusive SSFEM with a large number of random variables and higher order expansions. Another difficulty of this approach is the need for intrusive adjustments in the associated deterministic PDE solver. Thus the existing deterministic FEM solver cannot be readily utilized; this makes the intrusive approach more complicated and demanding. To overcome this challenge, in this thesis the stochastic system assembly procedure is simplified by directly employing FEniCS deterministic assembly routines [32].

The implementational steps of intrusive SSFEM system assembly can be summarized as:

- Calculate and store the non-zero $\mathcal{C}_{i j k}$ terms, i.e., moments of multidimensional polynomials and their respective indices $i, j$ and $k$. This requires the computation of multidimensional inner products which can be obtained using one-dimensional moments and multi-indices [9] (see Appendix B.2 for more details on multi-index). In this thesis the routines adopted from UQ Toolkit [29] are employed to compute the $\mathcal{C}_{i j k}$ terms (see Appendix B.2 for further details on the implementation along with code snippets).

- The intrusive system matrix $\mathscr{A}$ is assembled using the deterministic system matrices $\hat{\mathbf{A}}_{i}$. For each of the input PCE index $i$ in Eq. (2.32), the deterministic finite element assembly routines from an existing FEM package can be used to assemble $\hat{\mathbf{A}}_{i}$ (see Algorithm 12 in Appendix B.2 for details).

- For the PCE indices $i, j$ and $k$ in Eq. (2.32), those matches with the pre-calculated nonzero $i, j$ and $k$ indices of $\mathcal{C}_{i j k}$, multiply the $i^{t h}$ assembled deterministic matrix $\hat{\mathbf{A}}_{i}$ with the respective $C_{i j k}$ and store it at the respective $[j, k]^{t h}$ position of stochastic matrix $\mathscr{A}$.

Similar steps can be utilized to assemble the right hand side vector $\mathscr{F}$. Further details on the FEniCS-based implementation of intrusive SSFEM along with the code snippets for each major step in the process are presented in Appendix B.2. 


\subsubsection{Non-Intrusive SSFEM Approach}

In this section, the PCE based non-intrusive spectral projection (NISP) approach is employed [9, 79, 108]. In the NISP approach, the Galerkin projection is directly performed on the PCE of the solution process given in Eq. (2.22). The orthogonality properties of PCE basis polynomials are utilized to compute the expansion coefficients of the solution process $[1,9]$ as,

$$
\begin{aligned}
\hat{\mathbf{u}}_{k} & =\frac{\left\langle\mathbf{u}(\theta) \Psi_{k}(\boldsymbol{\xi})\right\rangle}{\left\langle\Psi_{k}^{2}(\boldsymbol{\xi})\right\rangle} \\
& =\frac{1}{\left\langle\Psi_{k}^{2}(\boldsymbol{\xi})\right\rangle} \int_{\Omega} \mathbf{u}(\theta) \Psi_{k}(\boldsymbol{\xi}) \mathrm{p}(\boldsymbol{\xi}) d \boldsymbol{\xi}
\end{aligned}
$$

The denominator in Eq. (2.36) can be evaluated analytically beforehand (see Appendix C for examples). Thus the major computational effort lies in the evaluation of the multidimensional integral in the numerator. In the past, random sampling, tensor product quadrature or sparse grid quadrature based approaches were employed to solve the above integral [9, 62, 79, 108, 111]. The number of sampling points for random sampling and tensor product quadrature based approaches drastically increases with the increasing number of random variables and thereby renders these approaches inefficient $[62,106,111]$. In such cases, the sparse grid quadrature (for example, Eq. (2.37)) can be used to reduce the number of the solution evaluation points while maintaining the same level of accuracy $[9,62,79,111,162]$.

$$
\hat{\mathbf{u}}_{k}=\frac{1}{\left\langle\Psi_{k}^{2}(\boldsymbol{\xi})\right\rangle} \sum_{i=1}^{N_{s}} \omega_{p_{i}} \mathbf{u}\left(\boldsymbol{\xi}_{i}\right) \Psi_{k}\left(\boldsymbol{\xi}_{i}\right)
$$

where $N_{s}$ is the number of quadrature point with nodes $\xi_{i}$ and corresponding weights $\omega_{p_{i}}$ (which incorporates $\operatorname{PDF} \mathrm{p}\left(\boldsymbol{\xi}_{i}\right)$ ). In the current implementation, the Smolyak sparse grid quadrature scheme is employed to evaluate the integral in the numerator of Eq. $(2.36)[62,111,162,189]$. For the sake of completeness we provide a minimal description of sparse grid techniques. Numerous articles are available in the literature on the formulation of Smolyak sparse grid and its applications to SSFEM [12,62,106,111, 162, 189]. 
To illustrate the implementation of sparse grid quadrature based NISP approach, consider the finite element discretization of a stochastic PDE given in Eq. (2.20). Using $\left\{\xi_{1}, \xi_{2}, \ldots, \boldsymbol{\xi}_{n_{s}}\right\}$ the following deterministic system is solved at $N_{s}$ sample points (in this case, sparse quadrature points) using an existing deterministic solver:

$$
\mathbf{A}\left(\boldsymbol{\xi}_{i}(\theta)\right) \mathbf{u}(\theta)=\mathbf{f}, \quad i=1, \ldots, N_{s} .
$$

The evaluated $\mathbf{u}(\theta)$ at each sample point $\boldsymbol{\xi}_{i}$ is used in Eq. (2.36) to calculate the PCE coefficients of the solution process.

The sparse grid quadrature rule to integrate a multidimensional function $\mathcal{F}=\mathbf{u}(\theta) \Psi_{k}(\boldsymbol{\xi})$ in the numerator of Eq. (2.36) can be constructed using the univariate quadrature rule $Q_{l}^{(1)} \mathcal{F}$ as follows $[12,162]$,

$$
Q_{l}^{(1)} \mathcal{F}=\sum_{q=1}^{N_{s}} \mathcal{F}\left(r_{l}^{q}\right) w_{p l}^{q},
$$

where the subscript $l$ of $Q$ is the level of quadrature and the superscript of $Q$ denotes the dimension $d$ (in this case $d=1$ ). $N_{s}$ is the number of quadrature points in the sparse grid and $r_{l}^{q}$ and $w_{p l}^{q}$ are the nodes and respective weights for the $l^{\text {th }}$ level sparse grid quadrature. The difference relation between two levels can be defined as [12]

$$
\Delta_{l}^{(1)} \mathcal{F}=\left(Q_{l}^{(1)}-Q_{l-1}^{(1)}\right) \mathcal{F}
$$

where $Q_{0}^{(1)} \mathcal{F}=0$ and $\Delta_{l}^{(1)} \mathcal{F}$ is also a quadrature formula with the nodes same as $Q_{l}^{(1)} \mathcal{F}$ and weights are difference between those at the levels $l$ and $l-1$. The set of nodal point for onedimensional quadrature $Q_{l}^{(1)} \mathcal{F}$ is denote by

$$
\Theta_{l}^{(1)}=\left\{r_{l}^{1}, \ldots, r_{l}^{N_{s}}\right\}
$$

For illustration of difference relation in Eq. (2.40), consider an example from [12]. The quadrature rule $Q_{1}^{(1)}$ for $d=1$ and $l=1$ with the nodes $\Theta_{1}^{(1)}=\{0,1\}$ and weights $=\{0.5,0.5\}$. 
Similarly the quadrature rule $Q_{2}^{(1)}$ for $l=2$ with the nodes $\Theta_{2}^{(1)}=\{0,0.5,1\}$ and weights = $\{0.25,0.5,0.25\}$. Then the nodes for the difference rule $\Delta_{2}^{(1)}$ are same as $\Theta_{2}^{(1)}=\{0,0.5,1\}$, but the weights $=\{-0.25,0.5,-0.25\}$ are obtained from the difference $Q_{2}^{(1)}-Q_{1}^{(1)}$.

The sparse grid formula for $d$-dimensional integral at level $l$ can be written using the difference relationship as [12]

$$
Q_{l}^{(d)} \mathcal{F}=\sum_{\left|l^{\prime}\right| \leq l+d-1}\left(\Delta_{l_{1}}^{(1)} \otimes \cdots \otimes \Delta_{l_{d}}^{(1)}\right) \mathcal{F}
$$

where $l^{\prime}=\left(l_{1} \ldots l_{d}\right)$ with $\left|l^{\prime}\right|=\sum_{i=1}^{d} l_{i}$. The nodal set $\Theta$ for the sparse grid can be obtained using one-dimensional sparse nodal set $\Theta_{l}^{(1)}$ as [12]

$$
\Theta_{l}^{(d)}=\bigcup_{\left|l^{\prime}\right| \leq l+d-1}\left(\Theta_{l_{1}}^{(1)} \times \cdots \times \Theta_{l_{d}}^{(1)}\right)
$$

where $\bigcup$ denotes union of subsets.

For example, consider $\Theta_{1}^{(1)}, \Theta_{2}^{(1)}$ and $\Theta_{3}^{(1)}$ are the nodal sets at level $l=1,2$ and 3 and dimension $d=1$. Then the sparse grid nodal set for $d=2$ and $l=3$ can be written as [12]

$$
\begin{aligned}
\Theta_{l=3}^{(d=2)} & =\bigcup_{\left|l^{\prime}\right| \leq l+d-1}\left(\Theta_{l_{1}}^{(1)} \times \Theta_{l_{2}}^{(1)}\right) \\
& =\left(\Theta_{1}^{(1)} \times \Theta_{1}^{(1)}\right) \quad\left(l_{1}=1, l_{2}=1\right) \\
& \cup\left(\Theta_{1}^{(1)} \times \Theta_{2}^{(1)}\right) \cup\left(\Theta_{2}^{(1)} \times \Theta_{1}^{(1)}\right) \\
& \cup\left(\Theta_{1}^{(1)} \times \Theta_{3}^{(1)}\right) \cup\left(\Theta_{2}^{(1)} \times \Theta_{2}^{(1)}\right) \cup\left(\Theta_{3}^{(1)} \times \Theta_{1}^{(1)}\right) .
\end{aligned}
$$

Equivalent formulations of the Smolyak sparse grid using slightly different approach and also different notations can be found in the following articles [9,12, 162].

For the implementation of the multidimensional sparse grid shown in Eq. (2.42) the growth rule in one-dimensional quadrature given in Eq. (2.39) must be defined. The fully nested Clenshaw-Curtis abscissas [12,162] or the weakly-nested Gaussian abscissas [12,162] can be 
used. In the current implementation, we have used the Gaussian abscissas [12,162]. For illustration, Smolyak sparse grid with the Gaussian abscissas for the two-dimensional case with $l=3$ and $l=6$ are showed in Fig. 2.5 (see Appendix B.3 for more details on sparse grid construction with Gaussian abscissas). For a moderate dimension and the moderate level of quadrature, the sparse grid can drastically reduce the number of quadrature points compared to the tensor product grid $[62,106,111]$. However, for high dimensional cases, the number of quadrature points substantially increases. For example, with $d=20$ the number of quadrature points increase from 841 for $l=3$ to 1014809 for $l=6$. For additional details on the types of growth rules and their performance comparisons for non-intrusive SSFEM, refer the following articles and reference therein $[12,62,111,162]$.
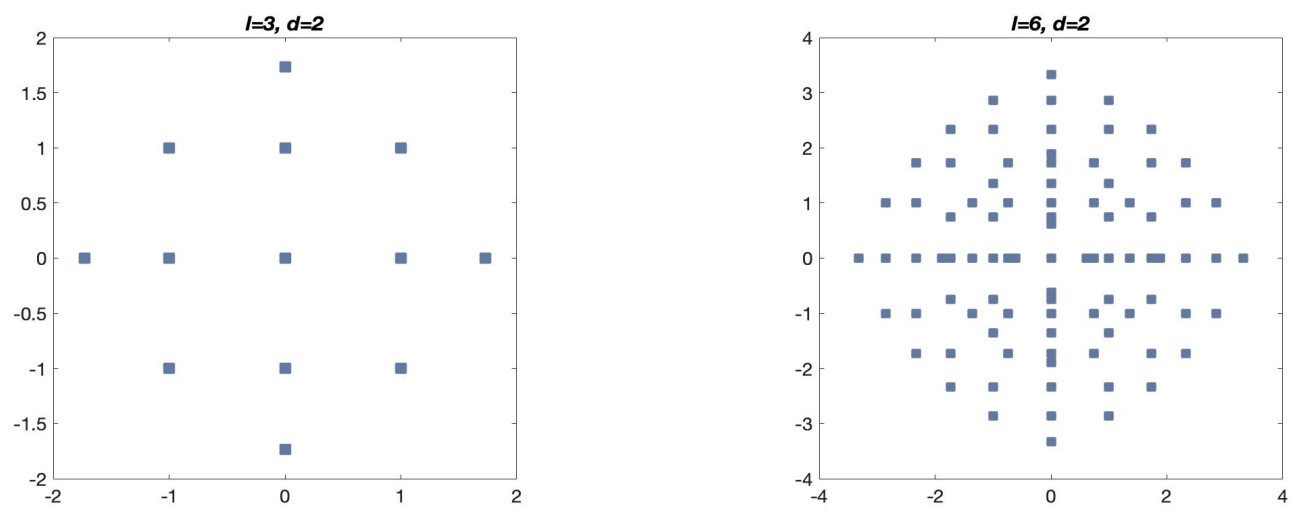

Figure 2.5: Number of quadrature points for a fixed $d=2$ with $l=3$ and $l=6$.

The main advantage of the non-intrusive approach is that we do not have to modify the underlying deterministic PDE simulation code to use this method. Instead, the repetitive deterministic system solves are required at each sample point. This can be performed by directly employing any existing deterministic finite element solver as a black-box. Hence, the implementation of non-intrusive approaches demands a mush less coding effort than the intrusive approach. This feature makes the non-intrusive approaches more attractive option over the intrusive approach $[101,106,113,116,118]$. In this thesis, the finite element solver from FEniCS FEM package $[32,188]$ is employed to compute solution at the required samples. Further details on the implementation of NISP approach with the code snippets are outlined in Appendix B.3. 


\subsection{Comparison of Intrusive and Non-Intrusive SSFEM}

To the author's best knowledge, there is no unanimous agreement in the UQ research community on which approach is superior: intrusive or non-intrusive method. Many articles suggest that the intrusive SSFEM method is more efficient concerning the accuracy and computational cost (albeit with substantial additional coding effort) as compared to the non-intrusive method for the following reasons $[64,72,113,118,119]$ :

- To achieve the same level of accuracy, the computational cost of repeated system solution (e.g., at numerous quadrature points) of non-intrusive approach is considerably higher than that of the intrusive system arising from the stochastic Galerkin approach [113].

- The error due to the finite representation in intrusive approach is minimum leading to an optimal accuracy [1]. On the other hand, the accuracy of the non-intrusive approach highly depends on the choice of the sampling (quadrature, collocation) points [9, 64, 116, 162]. For the large-scale applications where each deterministic simulation is computationally demanding the sampling-based non-intrusive approaches are computationally costly [113]. In such cases, the intrusive approach can be advantageous if an efficient intrusive system solver is employed [113].

In this section, we implement both intrusive (SG) and non-intrusive (NISP) SSFEM approaches to compare their performances. The focus is given to compare the accuracies of these approaches for non-Gaussian random process modeling the stochastic system parameters with increasing number of random variables $[182,183]$. In the numerous assessments performed in the past, attention is given to the uniform or Gaussian random variables [101, 113, 116]. However, comparative studies for non-Gaussian random processes modeling the stochastic system parameters are not widely available in the literature. Moreover, in this investigation, we conduct a detailed error analysis of the individual PCE coefficients along with the mean and variance of solution process to gain insights into the convergence behavior of both intrusive and non-intrusive approaches. This was lacking in the previous articles related to such 
assessments $[101,113,118]$. Note that, the adaptive PCE approaches reported in the literature [111,190-192] to lower the increased computational cost of the SSFEM approaches in high-dimensional stochastic spaces, are not considered in this thesis. Such adaptations can be exploited in both intrusive and non-intrusive setting [111,190-192]. Therefore, it may provide gains in both the approaches. But they are not pursued here although such studies will be worthy of future investigations.

Consider a two-dimensional steady-state flow through random media, modeled by a stochastic diffusion equation. This leads to a Poisson equation defined by a linear elliptic stochastic PDE as given in Eq. (2.14). The diffusion coefficient $c_{d}(\boldsymbol{x}, \theta)$ is modeled as a lognormal stochastic process obtained by an exponential of a Gaussian process having the mean $g(\boldsymbol{x})=0$, standard deviation $\sigma=0.4$ and the exponential covariance function $C_{\alpha \alpha}$ (given in Eq. (2.4)) with correlation lengths $b_{1}=b_{2}=1.0$. The lognormal process representation ensures that the diffusion coefficient $c_{d}$ remains positive over the entire domain. The physical domain with unit-square geometry is discretized using an unstructured finite element mesh with $N=133$ nodes and 264 linear triangular elements.

The PCE representations from Eq. (2.10) are used for the uncertain system parameters and the solution process. The procedure outlined in the Sec. 2.2.1 is followed to formulate the intrusive SSFEM system given in the form of Eq. (2.34). The PCE coefficients of the solution process are obtained by directly solving the intrusive system given in Eq. (2.34). Similarly, the non-intrusive procedure discussed in Sec. 2.2.2 is employed to obtain the solution coefficients from Eq. (2.36). The required deterministic samples for the sparse grid quadratures are computed by directly solving Eq. (2.38). Note that the results presented in this section are obtained by employing a direct serial solver for both intrusive and non-intrusive SSFEM.

In Fig. 2.6 and 2.7, the block-diagonal structures of intrusive system matrices are presented. In Fig. 2.6, $p_{u}$ is increased from 4 to 5 while keeping $L=3$ fixed. In Fig. 2.7, $L$ is increased from 4 to 5 while keeping $p_{u}=3$ fixed. Note that the effect of increasing the order of expansion is different from that of the number of random variables. The system matrix resulting from 

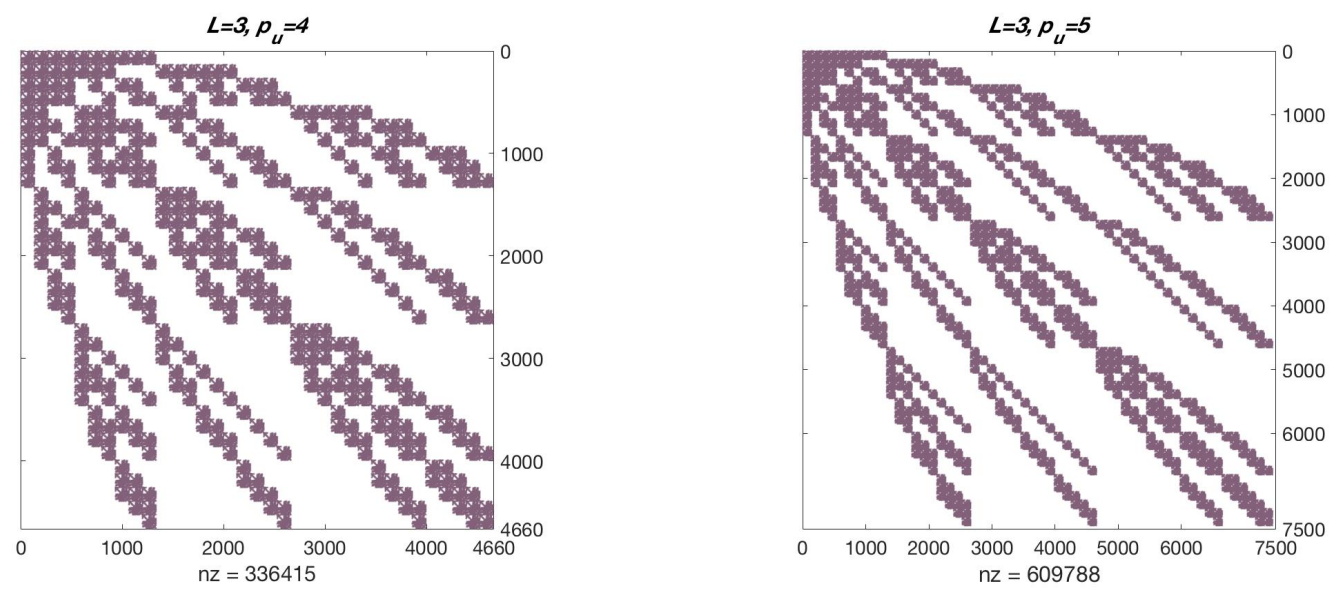

Figure 2.6: Block-sparse structures of the intrusive system matrices for a fixed mesh resolution with $L=3$ and $p_{u}=4,5$
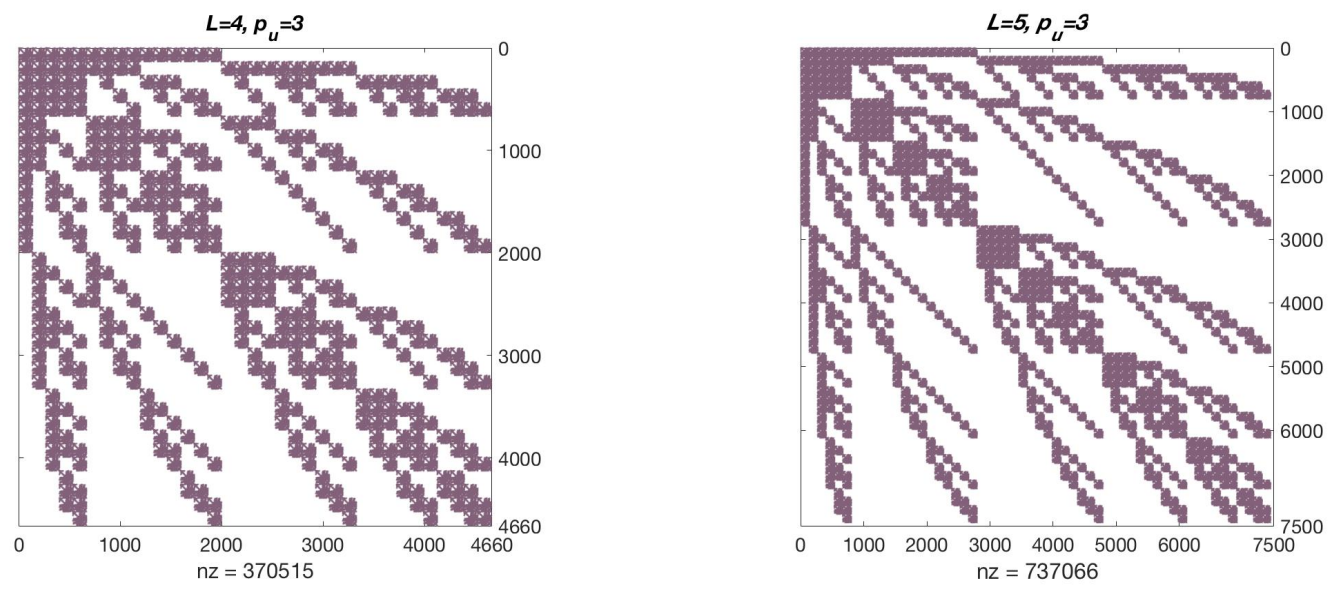

Figure 2.7: Block-sparse structures of the intrusive system matrices for a fixed mesh resolution with $p_{u}=3$ and $L=4,5$ 
varying $L$ have more nonzero entries compared to the one resulting from a similar change in $p_{u}$ as indicated by the number of nonzero entries $n z$ in Fig. 2.6 and 2.7. For example, with $L=5$ and $p_{u}=3$ we have 0.73 million nonzero elements compared to $L=3$ and $p_{u}=5$ which has 0.60 million nonzero elements. Therefore, an increase in the number of random variables may pose a more significant challenge compared to increasing order of expansion or the finite element mesh resolution concerning memory and computation requirements. However, both the order $p_{u}$ and dimension $L$ influence the size and structure of the stochastic assembly matrix, thereby, affecting the condition number of the system matrix [10,90].

To compare the performances of the intrusive approach with the non-intrusive approach, we consider the four different cases by selecting the number of random variables $L$ as 2, 3, 4 and 5. The second order PCE is used for input PCE ensuring non-Gaussian terms are included in the series expansion. The order of the input PCE is kept constant for all the experiments. The minimum order of PCE used in the expansion of solution process is $p_{u}=3$ which is higher than input PCE order, which captures the non-Gaussian effects [2,182]. Then $p_{u}$ is varied as 3,4 and 5 to perform error analysis in the lower order PCE coefficients.

Note that the intrusive SSFEM code employed for all the simulation in this exercise is validated with the SSFEM code developed by Khalil [178], which is validated using method of manufactured solution (see Appendix-D.3 in [178] for more details). Additionally, the intrusive SSFEM serial solver used here is validated with the parallel domain decomposition based solver employed in Chapter 3, which is validated against MCS in Appendix C. For non-intrusive case, the deterministic samples are simulated using the code validated against an analytical case in FEniCS/puffin [32,188]. The correctness of the quadrature points used to solve the integrals (in the numerator and the denominator of Eq. (2.36)) in the NISP approach are validated with the exact solution available for the $\left\langle\Psi_{k}^{2}(\xi)\right\rangle$.

The relative error norms are computed using $p_{u}=6$ in the intrusive SSFEM and used as the reference solution. For instance, the relative error in the $i^{\text {th }}$ order solution coefficient can be computed as $\left\|\left(\hat{\mathbf{u}}_{p_{u}=j}^{i}-\hat{\mathbf{u}}_{p_{u}=6}^{i}\right)\right\| /\left\|\hat{\mathbf{u}}_{p_{u}=6}^{i}\right\|$ with $j=3,4$ and 5 where $\|$.$\| denotes \mathrm{L}_{2}$ norm. 
For each case, the simulation is restricted to the maximum order of $p_{u}=6$. This is because we were not able to fit the system matrix for the intrusive approach for the higher-order cases (i.e., $p_{u}>6$ ) in the computer with quad-core processor and 16 gigabytes (GB) of random access memory (RAM) used for all the simulations.

The relative error norms in $0^{\text {th }}, 1^{\text {st }}$ and $2^{\text {nd }}$ order solution coefficients with $L=2,3,4$ and 5 for both intrusive and non-intrusive SSFEM are plotted in Figs. 2.8 to 2.11. In the intrusive approach, as the order of expansion of the solution process $p_{u}$ increases, the relative error in the lower order solution coefficients decreases. For example, if $p_{u}$ increased from 3 to 4 , the error in the lower order expansion terms, i.e., for $p_{u}=0,1$ or 2 , decreases as expected. Similarly in the non-intrusive approach, as the level of quadrature $l$ increases, the relative error in the individual solution PCE coefficients decreases. Note that, in the non-intrusive approach the solution coefficients are independent of each other as opposed to the intrusive approach, where the error in the lower order solution coefficients decrease as we include the higher-order expansion terms.

For the cases with lower order solution coefficients and a few random variables, for instance, $0^{\text {th }}$ order with $L=2$ presented in Fig. 2.8, the relative errors are similar in both intrusive and non-intrusive approaches with $l=p_{u}$. For the higher-order solution coefficients with a large number of random variables, for instance, the $3^{\text {rd }}$ order coefficient with $L=3$ presented in Fig. 2.9, the relative error is less in the case of intrusive approach compared to non-intrusive approach for $p_{u}=l$. The difference between the relative errors for the intrusive and nonintrusive approach grows as the $p_{u}$ and the $L$ increases. Therefore, in order to achieve the same level of accuracy in the PCE coefficients of the solution process, we need a higher level of the sparse quadrature in non-intrusive approach compared to the order of expansion in the intrusive approach (i.e., $l>p_{u}$ ). For example, in the case of $1^{\text {st }}$ order solution coefficient with 4 random variables presented in Fig. 2.10, to achieve the same level of accuracy as intrusive approach with $p_{u}=3$, we need the fourth level of quadrature. Similarly, in the case of $2^{\text {nd }}$ order solution coefficient with 5 random variables shown in Fig. 2.11, to achieve the same level of accuracy as in intrusive approach with $p_{u}=3$, we need the fifth level of quadrature. 

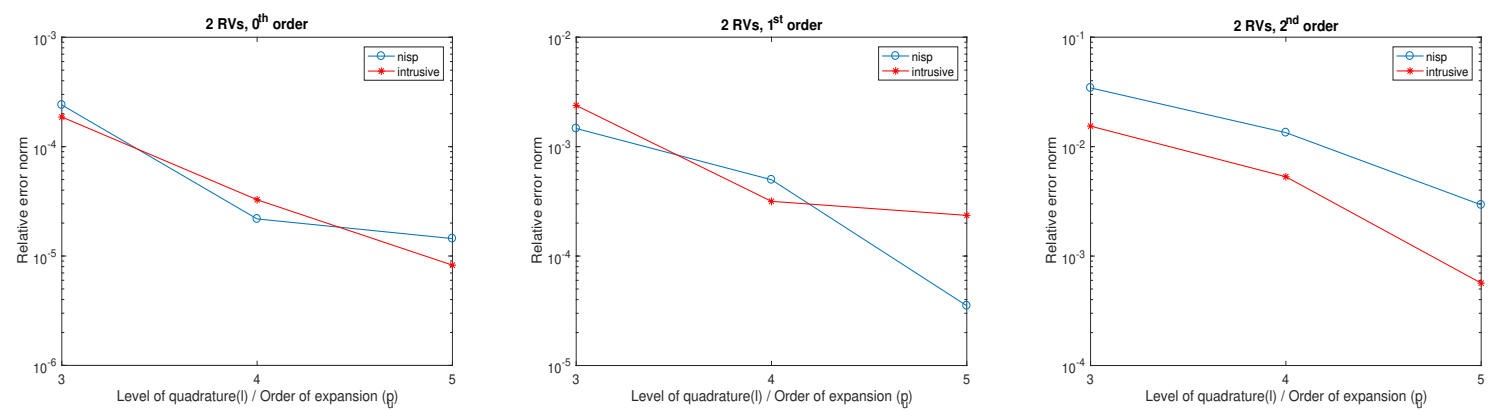

Figure 2.8: Error norms in $0^{t h}, 1^{\text {st }}$ and $2^{\text {nd }}$ order PCE coefficients for $L=2$.
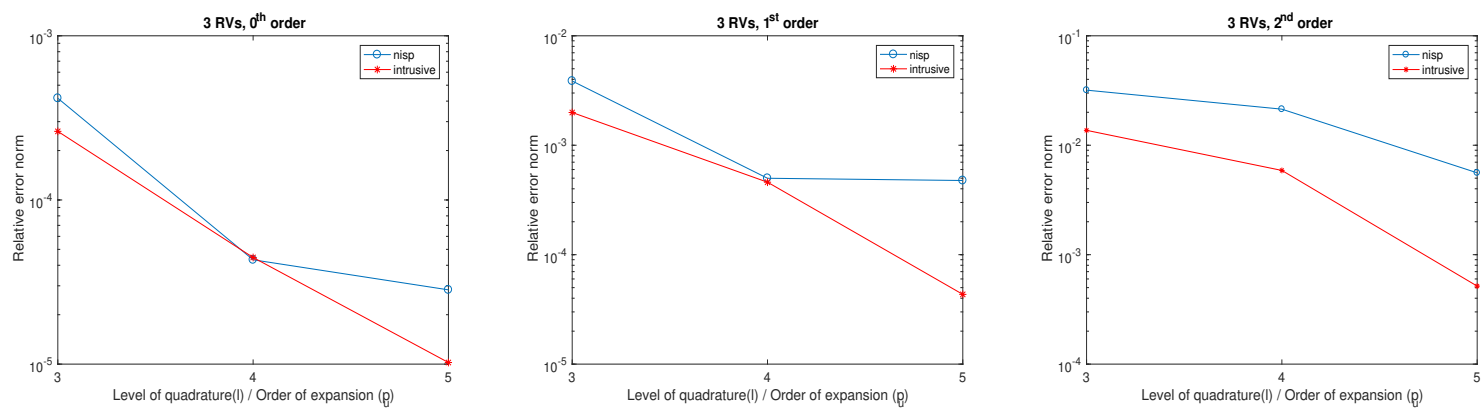

Figure 2.9: Error norms in $0^{t h}, 1^{\text {st }}$ and $2^{\text {nd }}$ order PCE coefficients for $L=3$.
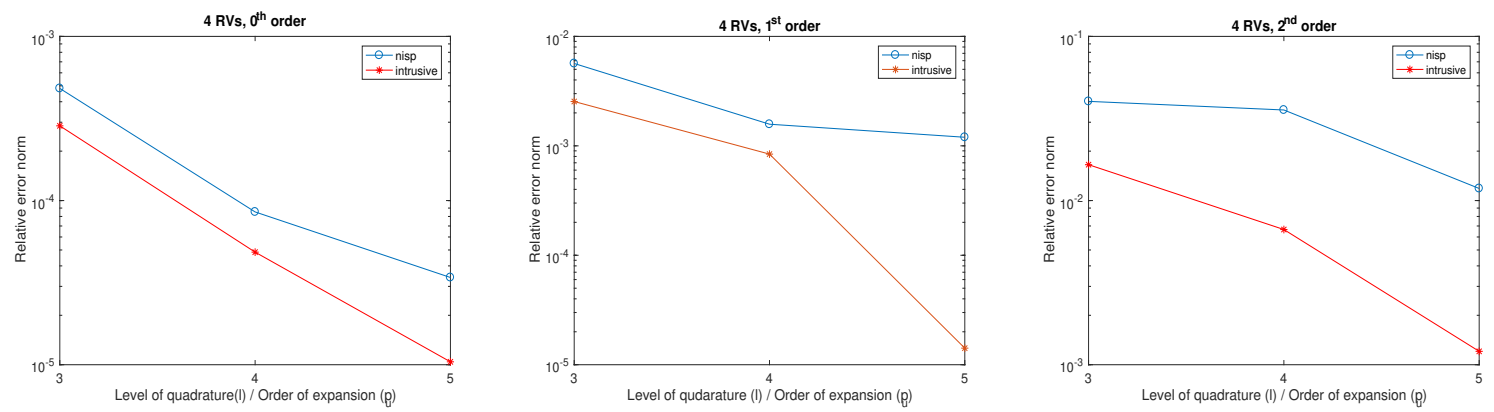

Figure 2.10: Error norms in $0^{\text {th }}, 1^{\text {st }}$ and $2^{\text {nd }}$ order PCE coefficients for $L=4$.
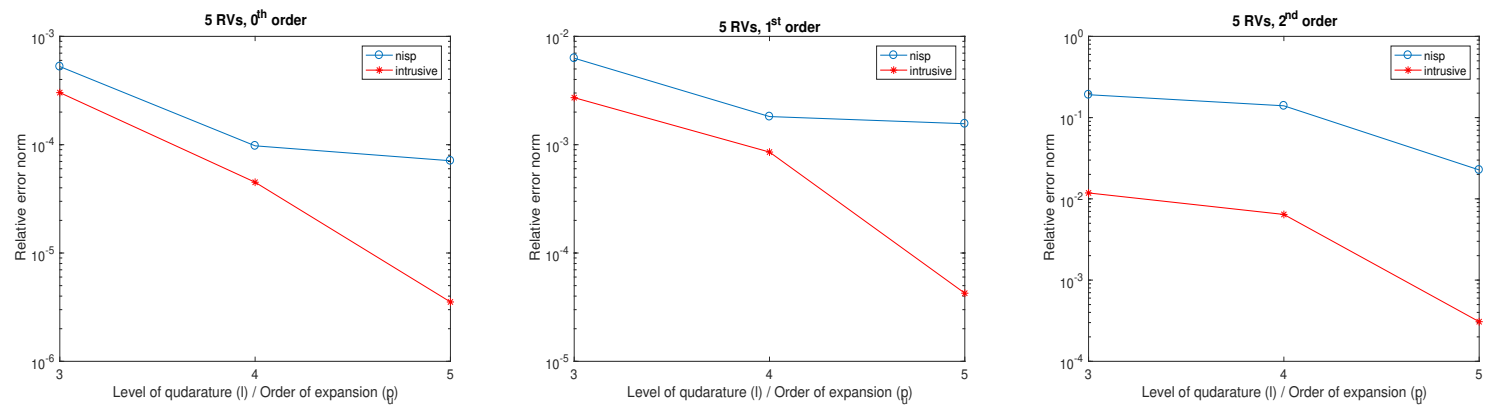

Figure 2.11: Comparison of relative error norms between intrusive and non-intrusive SSFEM in $0^{\text {th }}, 1^{\text {st }}$ and $2^{\text {nd }}$ order PCE coefficients for $L=5$. 

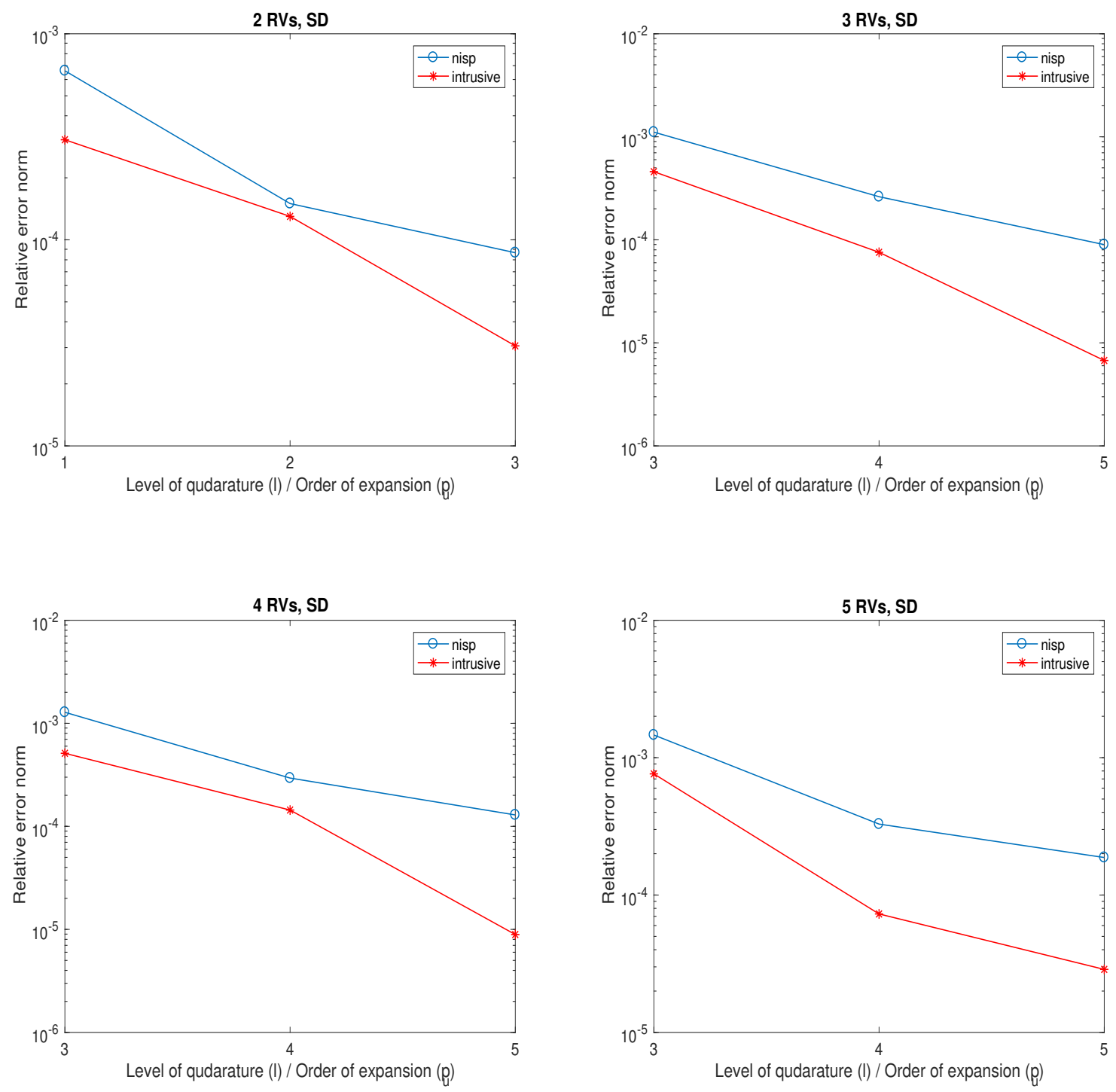

Figure 2.12: Comparison of relative error norms between intrusive and non-intrusive SSFEM in the standard deviation (computed for all nodes) of the solution process for $L=2,3,4$ and 5 . 
For the same cases discussed above, the relative error norms in the standard deviation $\sigma_{u}$ of the solution process (for all nodes) are shown in Fig. 2.12. Also, for $L=4$ the error surface plots in the SD of the solution process using intrusive with $p_{u}=3,4$ and 5 and NISP with $l=3,4$ and 5 are compared in Fig. 2.13. These plots also suggest that, for the same level of accuracy in the standard deviation of the solution process with the intrusive SSFEM approach, we need a higher level of quadrature to solve the integral involved in the non-intrusive approach. Therefore, to model the uncertainty as a non-Gaussian stochastic process characterized by a large number of random variables we have to use the higher level sparse grid quadrature, i.e., $l>>p_{u}$. Consequently, we need a large number of samples in the non-intrusive approach. For instance, if we increase the level of quadrature from 3 to 4 or 5 , there is a small increase in the number of quadrature points for low-dimensional cases, for example $L=5$. However, the number of samples for high-dimensional cases, for example $L=15$, increases quickly compared to the low-dimensional cases (i.e., $L=5$ ) as shown in Table 2.1.

\begin{tabular}{lrc}
\hline$N_{s}$ & $L=5$ & $L=15$ \\
\hline$l=3$ & 241 & 11561 \\
\hline$l=4$ & 781 & 120401 \\
\hline$l=5$ & 2203 & 259607 \\
\hline
\end{tabular}

Table 2.1: Number of sample (quadrature) points $N_{s}$ in the sparse grid with level $l$ and the number of random variables $L$.

In summary, from an implementational perspective the non-intrusive approach is favorable because one can directly employ any existing deterministic solver as a black box to simulate the required samples. On the other hand, the intrusive approach demands additional coding efforts. However, as demonstrated later in this thesis, the stochastic assembly procedure employed for intrusive SSFEM can utilize the readily available deterministic finite element assembly routines (such as FEniCS) which can substantially reduce the coding efforts. Also, as demonstrated above, for a large number of random variables and a high order of PCE, the intrusive approach has better control over the error in the individual solution coefficients compared with the nonintrusive approach. Therefore, for practical applications which may require modeling of non- 

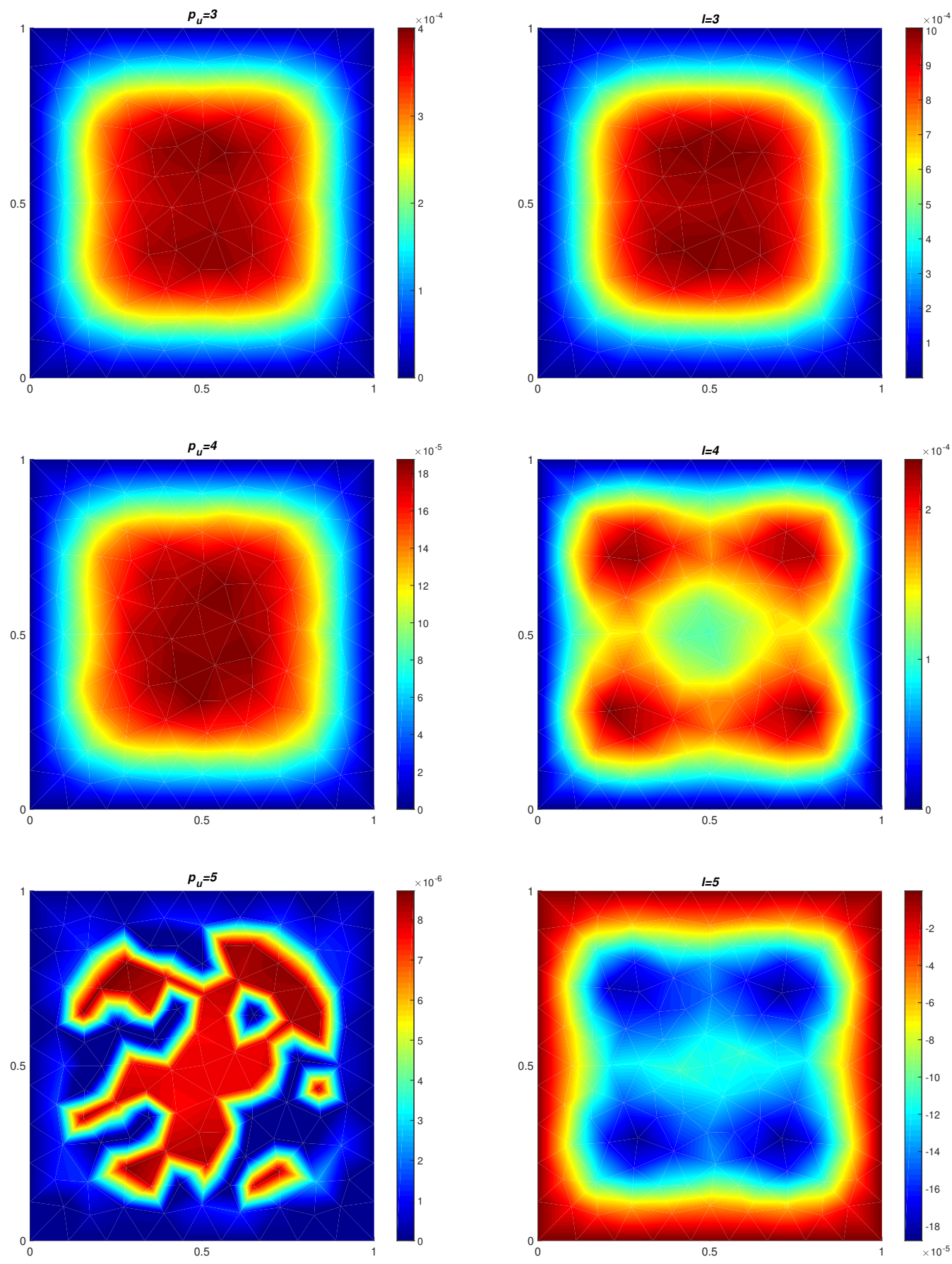

Figure 2.13: Comparison of error surface plots between intrusive and non-intrusive SSFEM for $L=4$ in the standard deviation of the solution process using $p_{u}=3,4,5$ and $l=3,4,5$ with respect to standard deviation of the solution process using $p_{u}=6$ and $l=6$ 
Gaussian (for example, lognormal process) system parameters requiring with large-number of random variables, the intrusive approach can be advantageous.

Despite the fact that the PCE based approaches (both intrusive and non-intrusive) can outperform the other approaches for many applications, they pose the following challenges:

- The PCE based approaches have difficulty in capturing sudden changes in the response (solution process), for example, predicting shock dynamics in the presence of uncertainties [192$194]$ and responses of highly nonlinear aeroelastic systems which show an abrupt jump to a higher period limit cycle oscillations [101, 105, 193, 195].

- The PCE solution has non-uniform convergence and tends to break down for long-time integration which is called the long-term degeneracy problem [101, 105, 191, 192, 196].

To address these challenge various adaptive PCE methods are developed [190-192, 194, 197]. One such method is adaptive multi-element generalized PCE [192,194]. The main idea of the this method is to adaptively decompose the space of random inputs into multiple elements and subsequently employ PCE at the element level. Similar technique but a nonintrusive multielement PCE formulation is employed in [191] in order to predict the stochastic response in the presence of discontinuities in the random space. In [197], a multi-resolution analysis based on multi-wavelet basis is applied to tackle uncertainty propagation in the complex and multidimensional stochastic problems. They conclude that the adaptive refinement of the multi-wavelet basis provides an attractive means to effectively tackle problems with steep or discontinuous dependence on random data. The nonintrusive B-spline stochastic projection method is developed in order to capture the sharp discontinuities along with the long term periodic behavior in [198]. In another approach by Gerritsma et al. [199], as time progresses the new stochastic variables are defined and corresponding orthogonal polynomials are constructed to alleviate long-term degeneracy issue. An algorithm based on constant phase interpolation was employed in [94, 105] to deal with the long time degeneracy problems. 
Although we can readily accommodate the increased number of samples due to a large number of random variables in the non-intrusive approach, there is a substantial increase in the number of sample evaluations for the non-Gaussian input making it computationally costly compared to the intrusive approach. However, the memory required to assemble and solve the intrusive system increases as we increase the number of random variables. Therefore, for a computer with a fixed random access memory (RAM), there is an upper limit to the size of intrusive system we can accommodate. Nonetheless, if we can handle the increasing intrusive system size by distributing it among multiple nodes and employ an efficient parallel solver, we can get the solution coefficients quickly compared to the non-intrusive approach for the same level of accuracy. For these reasons, in this research, we have focused on: (a) the development of scalable domain decomposition solvers for intrusive SSFEM for two and three-dimensional stochastic PDEs and (b) an efficient parallel implementation of these solvers utilizing highperformance computing (HPC) to solve the problems with high resolution in both spatial and stochastic domains. Developing scalable solvers to tackle stochastic PDEs using SSFEM is an active area of research as evidenced by many articles published in the last couple of decades $[24,90,148,152-156,169,170,173-175]$. 


\section{Chapter 3}

\section{Domain Decomposition of Stochastic PDEs}

\section{in Two Dimensions: Adaptations to Large}

\section{Random Variables}

This chapter is primarily based on the article published by the author [19] but also includes some new results. The enormous growth in high-performance computing (HPC) and their widespread of availability has inspired research in the field of domain decomposition (DD) based scalable solvers for deterministic PDEs [121, 125, 128, 139, 200]. Another reason for the popularity of DD techniques is their efficient parallelism and superior convergence $[128,139]$. In the context of a system of linear equations, the domain decomposition methods are often used as a preconditioner for the iterative solvers such as the conjugate gradient methods (CGM) or the method of generalized minimum residual (GMRES).

In the past, most of the research on DD based iterative solvers is centered on deterministic PDEs [26, 121, 125, 128, 139, 141, 200, 201]. However, the DD solvers are not yet widely exploited for the stochastic PDEs. This is because of the complexities arises in the numerical modeling of stochastic PDEs. For instance, intrusive SSFEM results in a spatially coupled system of linear equations in conjunction with an additional block coupling structure along the 
stochastic dimension [90,182]. Therefore, to efficiently tackle stochastic PDEs, it is essential to extend the deterministic DD algorithms tailored to fit such applications [17, 18, 23, 24].

The DD methods are classified into two groups. (a) overlapping methods ( e.g., Schwarz methods) and (b) non-overlapping methods (substructuring methods) $[125,128,139,200]$. In the context of overlapping DD methods, smaller problems are alternately solved on each of the overlapping subdomains. This is followed by the propagation of the interior solution onto the artificial boundary $[124,128]$. On the other hand, in the non-overlapping DD methods, the problem is formulated on each subdomain separately, and then the local degrees of freedom are divided into interior and interface components. This is followed by a global assembly and algebraic manipulation by which the original system is reduced to the Schur complement system $[125,128,139]$. Subsequently, the global interface unknowns are computed by solving the Schur complement system and then the local interior unknowns are obtained by solving a local interior system simultaneously on each subdomain $[125,128,139,200]$. Depending on the procedure employed to solve the Schur complement system, the non-overlapping DDM are grouped into the direct substructuring and the iterative substructuring methods [125, 128, 139, 200].

In the direct substructuring method, the Schur complement system is solved using direct solvers. This method is effective for a small-scale systems but computationally expensive for a large-scale system $[128,133,139,150]$. Therefore, the iterative substructuring methods are widely employed techniques in the structural mechanics community [140-142, 150]. The iterative substructuring techniques are further categorized into the primal and dual methods $[132,139]$. In the primal methods, the continuity conditions are strongly enforced by global assembly along the interfaces $[24,132,139,141]$. This category contains balancing domain decomposition (BDD) [123, 144], balancing domain decomposition by constraints (BDDC) and their variants $[123,141,145]$. On the other hand, in the dual methods, the continuity conditions are weakly enforced by Lagrange multipliers along the interfaces [146, 147]. This category includes the finite element tearing and interconnect (FETI) and the dual-primal FETI (FETI-DP) and its variants $[131,147,149]$. In this thesis, we concentrate on the non-overlapping DDM, 
particularly the iterative substructuring techniques with the primal and dual-primal approaches, due to their excellent numerical and parallel scalabilities [128,139,200].

In the deterministic settings, the condition number $(\ell)$ for the Schur complement matrix (S) appearing in the context of iterative substructuring techniques is $[125,128,139]$

$$
k(\mathbf{S})=\mathcal{O}(1 / h H)
$$

where $H$ is the size of the subdomain and $h$ is the size of the finite element. For numerous second-order deterministic PDEs, the condition number bound of the resulting Schur complement matrix is better than the original system matrix, which typically has a condition number on the order of $1 / h^{2}$. However, as the number of subdomains increases, the resulting Schur complement matrix becomes ill-conditioned. Therefore, a preconditioner $\left(\mathbf{M}^{-1}\right)$ is necessary to solve the Schur complement system efficiently [128, 139, 150,200].

In the past, various preconditioners were employed to improve the condition number of the Schur complement system. However, the preconditioners with coarse grid corrections are most widely adopted $[125,128,139,200]$. This is because, the coarse grid can provide an efficient mechanism for global communication of information, and therefore can reduce the condition number of preconditioned Schur complement system [125,128,139,200]. The condition number bound for the preconditioned Schur complement system with a vertex-based coarse grid in two dimensions is as shown in [26, 128, 139]

$$
k\left(\mathbf{M}^{-1} \mathbf{S}\right) \leq C(1+\log (H / h))^{2}
$$

where $C$ is a constant independent of the mesh size $h$ and subdomain size $H$. These bounds are quite satisfactory compared to the one-level preconditioners, where the condition number typically depends on $H^{-2}[125,128,139,200]$.

In the following sections, we review the domain decomposition of stochastic PDEs. First, the challenges with the existing DD algorithms are outlined. This is followed by the discus- 
sion on proposed algorithmic and implementational advances. In Sec. 3.1.1 an overview of non-overlapping DD method with emphasis on the formulation of extended Schur complement system is presented. It also covers the formulation of the two-level BDDC/NNC preconditioner [17]. In Sec. 3.1.2, we present the probabilistic version of dual-primal DD method [18].

\subsection{Domain Decomposition of Stochastic PDEs}

In the context of intrusive SSFEM, the extended (to the stochastic cases) non-overlapping domain decomposition method for stochastic PDEs was first formulated by Sarkar et al. [23] and later extended by Subber and Sarkar $[17,18]$ to quantify uncertainty in large-scale systems with a few random variables. One-level DD preconditioners, such as extended-lumped, extendedweighted-lumped and extended-Neumann-Neumann preconditioners were employed for the iterative solution of the extended Schur complement system in [17,24]. Similar to deterministic cases, the performance of the one-level preconditioner deteriorates with the number of subdomains and the size of the linear system arising in the stochastic cases [24]. The two-level Neumann-Neumann preconditioner for stochastic systems developed by combining local and global corrections showed excellent numerical scalability for the high-resolution finite element meshes in the cases of a few random variables [17,24]. This two-level DD method can be construed to be the probabilistic version of a type of $\operatorname{BDDC}[17,140,141]$. The probabilistic version of FETI-DP [131] based domain decomposition solver exhibited similar performance for the systems arising in intrusive SSFEM [18,24].

The intrusive PCE based non-overlapping DDM algorithms with preconditioned conjugate gradient method (PCGM) presented in $[17,18,178]$ showed excellent scalabilities for cases with high mesh resolution and few random variables. For uncertainty quantification (UQ) of practical engineering applications, which demands high resolution discretization in both spatial and stochastic dimensions, these solvers suffer from (a) the large memory requirement in order to assemble and store the subdomain-level stochastic finite element matrices. (b) The excessive 
computational effort needed in factorizing these local matrices to solve the global coarse problem and multiple subdomain-level local systems arising in the context of DDM-based PCGM. (c) Dependency on in-house stochastic finite element assembly codes which demand extensive coding efforts. Furthermore, the DDM solvers developed in $[17,18,178]$ were never tested for robustness and scalability on a large-scale computing cluster with thousands of cores.

In this chapter, the focus is to alleviate these concerns. The attention is given to the efficient implementation of algorithms for large-scale stochastic systems with a large number of random variables and the associated algorithmic extensions. To this end, the contributions made to overcome the above challenges are broadly categorized next.

- Three-level nested PCGM solvers are formulated for the global interface systems arising in the context of BDDC/NNC and FETI-DP. In these solver, the global fine (interface) problem is solved using PCGM algorithm with DDM based preconditioner (outer PCGM). The global coarse problem in the construction of two-level DDM preconditioner in BDDC/NNC (or in the operator in FETI-DP) is solved employing another PCGM algorithm with DDM-based one-level preconditioner (coarse PCGM). Multiple subdomain-level local problems associated with the outer PCGM and coarse PCGM iterations are solved while utilizing PETScbased sparse iterative solvers (inner PCGM) with the block-Jacob preconditioner [31].

- For the cases involving a large number of random variables, an efficient subdomain-level stochastic block matrix assembly procedure is developed. The new assembly routine optimizes the memory management via the recursive construction of the subdomain-level sparse blocks of the stochastic system matrices using the temporarily assembled subdomain-level blocks of deterministic system matrices. Using this stochastic assembly procedure, the DDM solvers are coupled with the FEniCS deterministic finite element package [32,177]. This coupling with FEniCS enables the DD based parallel solver for intrusive-SSFEM to tackle a variety of PDEs without the burden of in-house complex assembly code development $[9,106,108]$.

- Scalable and efficient implementation of the proposed solvers is achieved with respect to 
stochastic dimensions by incorporating the following features. First, the solvers utilize numerous sparse data-structures and routines from PETSc for commonly occurring subdomainlevel algebraic operations and local system solves in multi-level PCGM implementations. It minimizes the memory requirements and floating-point operations which are critical for simulation of SSFEM models with the large random variables. Second, the moments of multidimensional polynomials which require thousands of multidimensional inner products are obtained using routines developed by adapting procedures from UQ Toolkit [29] involving strictly one-dimensional moments [9]. Third, the computations of large sets of multidimensional PCE/KLE basis functions and the calculation of input PCE/KLE coefficients are automated using the routines developed by adapting functions from UQ Toolkit [29]. Furthermore, this article elaborates on the implementation aspects of the solvers which were lacking in previous articles related to these methods $[17,18,178]$. The algorithmic details are presented for completeness in order to assist computational scientists in implementing these methods for their applications.

All of the proposed changes are implemented in probabilistic BDDC/NNC [17] and FETIDP [18] solvers to efficiently handle high-dimensional stochastic systems. These solvers are implemented efficiently while relying on the popular scientific packages MPI, PETSc, FEniCS and UQTk rendering these solvers robust and scalable. Note that, a theoretical analysis of the condition number bound for the resulting intrusive SSFEM system matrix is not yet available in the literature, and no attempts are made in this thesis to perform such analysis. However, extensive numerical experiments concerning parallel and numerical scalabilities are conducted to investigate the performance of the solver thoroughly.

First, the performance analysis concerning the numerical scalability of the solvers, i.e., the number of PCGM iterations to solve the extended Schur complement system is measured with respect to the spatial mesh resolution and the number of subdomains. This study can help in understanding the condition number bounds of the preconditioned system matrix. It is considered crucial for the utility of the solver for the large-scale applications [24, 26, 136]. 
Secondly, the parallel scalability of the solvers is measured concerning the strong and weak scaling $[121,139]$. In the strong scalability test which is a measure of execution speed of the solver the global problem size is kept constant and the number of cores used to solve the problem is increased to reduce the total execution time. For the weak scalability test which is a measure of resolution, the problem size per subdomain is fixed and the global size of the problem is increased by adding more subdomains. In the weak scaling, the goal is to solve higher resolution problem using proportionally more cores in nearly a constant execution time. The parallel scalability of the solver can help us to understand the maximum size and minimum time required to solve the system using the available computational resources. Therefore, this study is crucial from a high-performance computing perspective.

Next, the scalability study with respect to the stochastic parameters such as the number of random variables and order of expansion (number of PCE terms) is also reported. Understanding behavior of the solver with respect to stochastic parameters is crucial for uncertainty quantification. This is because, as the number of PCE terms increase, the block-sparsity and the block-coupling structure of the underlying intrusive SSFEM system matrix changes. The effects are more significant in the cases of varying the number of random variables as compared to the order of expansions (refer the discussion and SSFEM system matrix presented earlier in Sec. 2.2.1). These aspects are different compared to the scalability studies commonly conducted for the deterministic FEM. This is because, in the deterministic setting, varying mesh resolution or the values of the system parameters, does not influence the sparsity pattern of the underlying system matrix. Therefore, the scalability plots concerning stochastic parameters provide understanding regarding the applicability of these solvers for the large-scale applications.

The numerical and parallel scalability studies on a large-scale computing cluster with thousands of cores is performed to demonstrate the utility of these solvers to the large-scale applications. Finally, the computational advantages of intrusive SSFEM equipped with scalable parallel solvers over non-intrusive SSFEM are demonstrated for the cases of large-scale discretized stochastic PDEs with a large number of random variables, characterizing non-Gaussian 
stochastic process. To the best knowledge of the author, these algorithms have not been applied previously for uncertainty quantification in large-scale stochastic systems at this scale.

\subsubsection{Non-Overlapping Domain Decomposition - the Primal Approach}

The finite element discretization of an elliptic stochastic PDE leads to a system of linear equations with stochastically varying system parameters and solution process given by [1],

$$
\mathbf{A}(\theta) \mathbf{u}(\theta)=\mathbf{f}
$$

where $\mathbf{A}(\theta)$ is called a random or stochastic system matrix, $\mathbf{u}(\theta)$ is the stochastic response vector and $\mathbf{f}$ is the deterministic force vector.

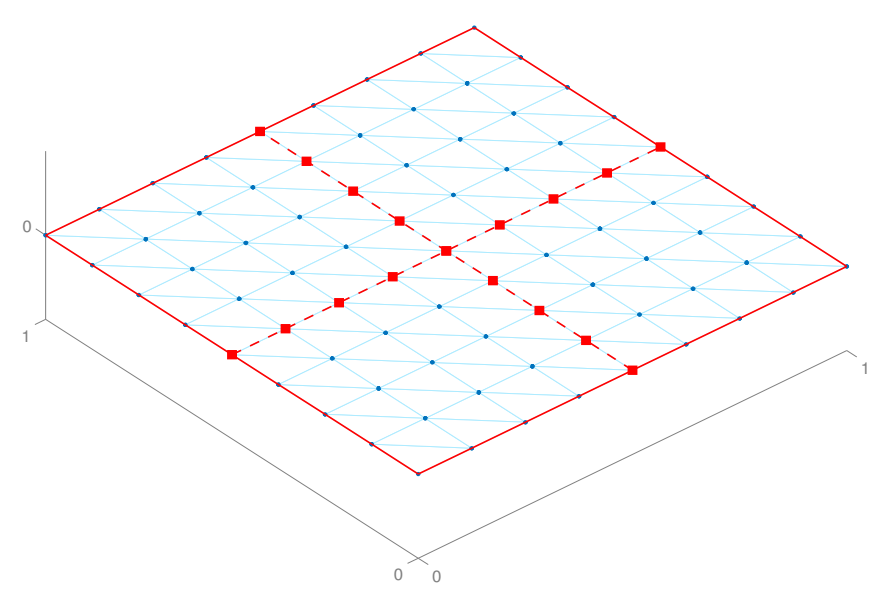

Figure 3.1: One-level mesh decomposition showing .interior $(I)$ and interface $(\Gamma)$ nodes.

To employ the domain decomposition method, the computational domain $D$ is partitioned into $n_{s}$ non-overlapping subdomains. The solution vector $\mathbf{u}^{s}(\theta)$ for each of the subdomain $s$, with $s=1,2, \ldots, n_{s}$, is divided into interior $\mathbf{u}_{I}^{s}(\theta)$ and interface $\mathbf{u}_{\Gamma}^{s}(\theta)$ vectors.

The subdomain-level equilibrium equation for a typical subdomain $s$ is written as [23,24, 
178],

$$
\left[\begin{array}{ll}
\mathbf{A}_{I I}^{s}(\theta) & \mathbf{A}_{I \Gamma}^{s}(\theta) \\
\mathbf{A}_{\Gamma I}^{s}(\theta) & \mathbf{A}_{\Gamma \Gamma}^{s}(\theta)
\end{array}\right]\left\{\begin{array}{c}
\mathbf{u}_{I}^{s}(\theta) \\
\mathbf{u}_{\Gamma}^{s}(\theta)
\end{array}\right\}=\left\{\begin{array}{c}
\mathbf{f}_{I}^{s} \\
\mathbf{f}_{\Gamma}^{s}
\end{array}\right\},
$$

where $\mathbf{u}_{I}^{s}$ is the interior solution vector corresponding to the interior nodes and $\mathbf{u}_{\Gamma}^{s}$ is the interface solution vector of the nodes shared by two or more adjacent subdomains as shown in Fig. 3.1.

The subdomain level blocks of the stochastic finite element matrix in Eq. (3.4) are expressed by using an $P_{\mathrm{A}}$-term PCE. The solution processes in Eq. (3.4) is represented using $P_{u}$ terms in PCE. These expansions are shown below,

$$
\mathbf{A}_{\alpha \beta}^{s}(\theta) \approx \sum_{i=0}^{P_{\mathrm{A}}} \overline{\mathbf{A}}_{\alpha \beta, i}^{s} \Psi_{i}(\theta), \quad \mathbf{u}_{\alpha}^{s}(\theta) \approx \sum_{j=0}^{P_{u}} \overline{\mathbf{u}}_{\alpha, j}^{s} \Psi_{j}(\theta),
$$

where $\left\{\Psi_{i}(\theta)\right\}$ are multidimensional orthogonal polynomials (the argument $\theta$ of $\left\{\Psi_{i}\right\}$ being dropped hereafter for notational simplicity), $\left\{\overline{\mathbf{u}}_{\alpha, j}^{s}\right\}$ are the deterministic PCE coefficients of the solution vector and $\left\{\overline{\mathbf{A}}_{\alpha \beta, i}^{s}\right\}$ are the PCE coefficient matrices. The $\alpha$ and $\beta$ denotes $\Gamma$ and $I$. Both $P_{\mathrm{A}}$ and $P_{u}$ are functions of the number of KLE terms and order of the polynomial chaos expansions of the input and output processes (refer [1,71] for details).

Using the expansions given in Eq. (3.5), the subdomain-level equilibrium equation in Eq. (3.4) is rewritten as $[23,24,178]$,

$$
\left(\sum_{i=0}^{P_{\mathrm{A}}} \Psi_{i}\left[\begin{array}{cc}
\overline{\mathbf{A}}_{I I, i}^{s} & \overline{\mathbf{A}}_{I \Gamma, i}^{s} \\
\overline{\mathbf{A}}_{\Gamma I, i}^{s} & \overline{\mathbf{A}}_{\Gamma \Gamma, i}^{s}
\end{array}\right]\right)\left(\sum_{j=0}^{P_{u}} \Psi_{j}\left\{\begin{array}{c}
\overline{\mathbf{u}}_{I, j}^{s} \\
\overline{\mathbf{u}}_{\Gamma, j}^{s}
\end{array}\right\}\right)=\left\{\begin{array}{c}
\mathbf{f}_{I}^{s} \\
\mathbf{f}_{\Gamma}^{s}
\end{array}\right\} .
$$

Using Galerkin projection $\left\langle. \Psi_{k}\right\rangle$ and then, performing global assembly leads to the following 
linear system of equations (see [23,24] and references therein for additional details):

$$
\left[\begin{array}{cccc}
A_{I I}^{1} & \ldots & 0 & A_{I \Gamma}^{1} R_{1} \\
\vdots & \ddots & \vdots & \vdots \\
0 & \ldots & A_{I I}^{n_{s}} & A_{I \Gamma}^{n_{s}} R_{n_{s}} \\
R_{1}^{T} A_{\Gamma I}^{1} & \ldots & R_{n_{s}}^{T} A_{\Gamma I}^{n_{s}} & \sum_{s=1}^{n_{s}} R_{s}^{T} A_{\Gamma \Gamma}^{s} R_{s}
\end{array}\right]\left\{\begin{array}{c}
u_{I}^{1} \\
\vdots \\
u_{I}^{n_{s}} \\
u_{\Gamma}
\end{array}\right\}=\left\{\begin{array}{c}
f_{I}^{1} \\
\vdots \\
f_{I}^{n_{s}} \\
\sum_{s=1}^{n_{s}} R_{s}^{T} f_{\Gamma}^{s}
\end{array}\right\}
$$

where

$$
\begin{gathered}
{\left[A_{\alpha \beta}^{s}\right]_{j k}=\sum_{i=0}^{P_{\mathrm{A}}}\left\langle\Psi_{i} \Psi_{j} \Psi_{k}\right\rangle\left[\overline{\mathbf{A}}_{\alpha \beta, i}^{s}\right],} \\
{\left[f_{\alpha}^{s}\right]_{k}=\left\langle\Psi_{k} \mathbf{f}_{\alpha}^{s}\right\rangle,} \\
u_{I}^{s}=\left(\overline{\mathbf{u}}_{I, 0}^{s}, \ldots, \overline{\mathbf{u}}_{I, P_{u}}^{s}\right)^{T}, \quad u_{\Gamma}=\left[\overline{\mathbf{u}}_{\Gamma, 0}, \ldots, \overline{\mathbf{u}}_{\Gamma, P_{u}}\right]^{T},
\end{gathered}
$$

where $u_{I}^{s}$ and $u_{\Gamma}$ are the vectors containing the PCE coefficients of the interior and interface unknowns respectively; and the subscript $\alpha$ and $\beta$ are for interior $(I)$ and interface $(\Gamma)$ nodes. The stochastic restriction operator $R_{s}$ is obtained from deterministic restriction operators $\mathbf{R}_{s}$ as

$$
R_{s}=\operatorname{blockdiagonal}\left(\mathbf{R}_{s, 0}, \ldots, \mathbf{R}_{s, P_{u}}\right)
$$

The $\mathbf{R}_{s, j}$ with $j=\left\{0,1, \ldots, P_{u}\right\}$, acts as scatter or gather operators between global and local components of the deterministic interface solution vectors as $[17,140]$,

$$
\begin{aligned}
& \mathbf{u}_{\Gamma, j}^{s}=\mathbf{R}_{s, j} \mathbf{u}_{\Gamma, j}, \\
& \mathbf{u}_{\Gamma, j}=\mathbf{R}_{s, j}^{T} \mathbf{u}_{\Gamma, j}^{s} .
\end{aligned}
$$




\section{Extended Schur Complement System}

Using Gaussian elimination technique to eliminate interior degrees of freedom from Eq. (3.7) leads to the extended (to the stochastic case) Schur complement system as shown below [17,23]:

$$
\begin{gathered}
S u_{\Gamma}=g_{\Gamma}, \\
S=\sum_{s=1}^{n_{s}} R_{s}^{T}\left[A_{\Gamma \Gamma}^{s}-A_{\Gamma I}^{s}\left[A_{I I}^{s}\right]^{-1} A_{I \Gamma}^{s}\right] R_{s}=\sum_{s=1}^{n_{s}} R_{s}^{T} S_{s} R_{s}, \\
g_{\Gamma}=\sum_{s=1}^{n_{s}} R_{s}^{T}\left[f_{\Gamma}^{s}-A_{\Gamma I}^{s}\left[A_{I I}^{s}\right]^{-1} f_{I}^{s}\right]=\sum_{s=1}^{n_{s}} R_{s}^{T} g_{\Gamma s} .
\end{gathered}
$$

where $S$ is the global extended Schur complement matrix and $g_{\Gamma}$ is the corresponding right hand side vector. Note that the extended Schur complement system is amenable to parallel implementation as each subdomain-level local Schur complement matrix $S_{s}$ and corresponding $g_{\Gamma s}$ can be solved concurrently.

The global interface solution coefficients $u_{\Gamma}$ are obtained by solving the extended Schur complement system in parallel. Consequently, they are used to obtain the local interior solution coefficient $u_{I}^{s}$ on each subdomain independently as

$$
A_{I I}^{s} u_{I}^{s}=\left(f_{I}^{s}-A_{I \Gamma}^{s} R_{s} u_{\Gamma}\right) .
$$

The schematic representation of the first four PCE coefficients of the solution process $u$ are displayed in Fig. 3.2. Here the horizontal axis represents the spatial discretization and the vertical axis shows the stochastic expansion (PCE coefficient number).

\section{Iterative Solution of the Extended Schur Compliment System}

As discussed in the previous sections, the original sparse system in Eq. (3.3), can be recast as a much smaller but denser interface system represented by the extended Schur complement 


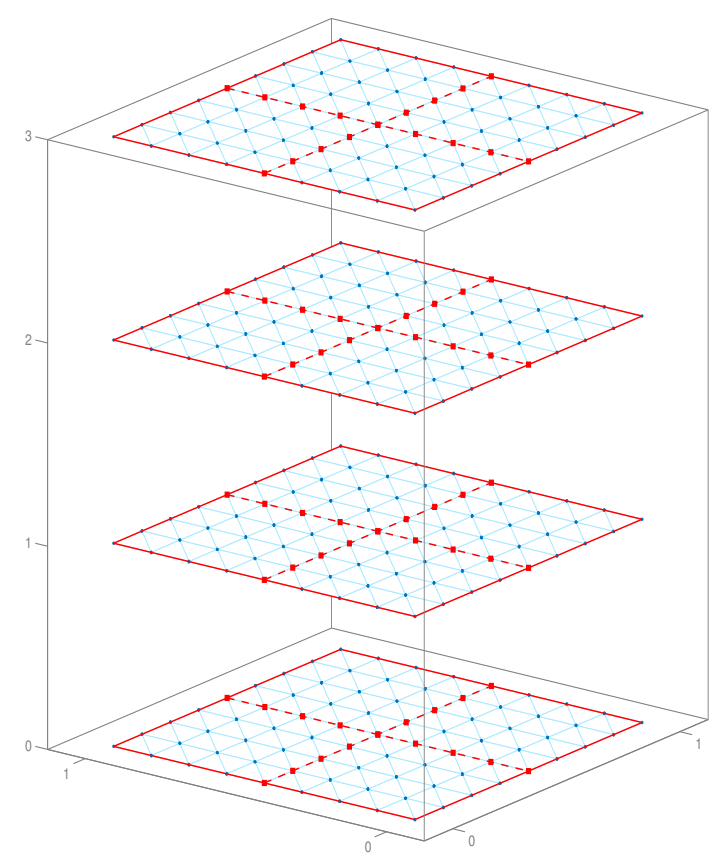

Figure 3.2: Schematic of first four PCE coefficients of the solution $\mathbf{u}$.

system given in Eq. (3.14). The condition number of the Schur complement matrix is better than the original stiffness matrix $[124,128]$, and this is believed to extend to the stochastic case $[24,156]$.

For the iterative solution, the following preconditioned Schur complement system is used [17]

$$
M^{-1} S u_{\Gamma}=M^{-1} g_{\Gamma},
$$

where $M^{-1}$ is the preconditioner which improves the condition number of the system [17,150]. Various preconditioners are available to improve the convergence of the iterative solution of the Schur complement system [24, 140, 201]. In this work, we employ the two-level NeumannNeumann preconditioner with coarse grid (NNC) [140] which is a probabilistic version of BDDC. In its general form, the two-level preconditioner is expressed as [17,24],

$$
M^{-1}=\sum_{s=1}^{n_{s}} R_{s}^{T}\left[M_{s}\right]^{-1} R_{s}+R_{0}^{T}\left[M_{0}\right]^{-1} R_{0} .
$$


The BDDC/NNC preconditioner consists of two parts: (a) the local part, corresponding to a fine problem $\left(M_{s}^{-1}\right)$ on each of the subdomains and (b) the global part, relating to a coarse problem $\left(M_{0}^{-1}\right)$ on the entire domain. $R_{s}$ acts as fine scale restriction operator and $R_{0}$ represents the coarse scale restriction matrix. The fine and coarse grid components of the two-level preconditioner provide the mechanism to reduce error locally (on each subdomain) and globally (on entire domain) and thereby reduce the iteration counts of the solver [17,24].

In the current work, we have chosen the steady state diffusion equation as our test case. This phenomenon is represented by a linear second-order elliptic PDE, which leads to a symmetric and positive definite global Schur complement matrix [125, 128, 200]. Therefore, the preconditioned conjugate gradient method (PCGM) is used to compute the interface solution coefficients. However, in general case with unsymmetric system matrix for instance, the convection-diffusion equations, preconditioned generalized minimal residual method (GMRES) can be used [21,24]. Once the interface solution coefficients from Eq. (3.18) are available, Eq. (3.17) can be solved on each subdomain in parallel to compute the interior solution coefficients [17,24].

\section{Neumann-Neumann with Coarse Grid Preconditioner}

The construction of BDDC/NNC preconditioner requires the second-level of partitioning of the degrees of freedom as reviewed next [17,24,140]. The subdomain-level interface solution coefficients $u_{\Gamma}^{s}$ are further divided into $u_{r}^{s}$ and $u_{c}^{s}$ as follows [17,24, 140]:

$$
\left\{\begin{array}{l}
u_{r}^{s} \\
u_{c}^{s}
\end{array}\right\}=\left\{\begin{array}{l}
R_{s}^{r} \\
R_{s}^{c}
\end{array}\right\} u_{\Gamma}^{s}
$$

where $u_{c}^{s}$ are the corner nodes, i.e. the nodes shared among three or more subdomains plus the nodes at the ends of interface edges and $u_{r}^{s}$ is remaining interface nodes, i.e. the nodes shared between two subdomains (excluding those at the ends of interface edges). The schematic representation of the first four PCE coefficients for the solution process with two-level mesh decomposition including the corner nodes and remaining interface nodes is shown in Fig. 3.3. 
$R_{s}^{r}$ and $R_{s}^{c}$ are the Boolean operators used to separate the interface solution coefficients into the remaining and corner solution coefficients respectively. They are obtained as follows [24, 140]:

$$
\begin{aligned}
& R_{s}^{r}=\operatorname{blockdiagonal}\left(\mathbf{R}_{s, 0}^{r}, \ldots, \mathbf{R}_{s, P_{u}}^{r}\right) \\
& R_{s}^{c}=\operatorname{blockdiagonal}\left(\mathbf{R}_{s, 0}^{c}, \ldots, \mathbf{R}_{s, P_{u}}^{c}\right)
\end{aligned}
$$

where the $\mathbf{R}_{s, j}^{r}$ and $\mathbf{R}_{s, j}^{c}$ with $j=\left\{0,1, \ldots, P_{u}\right\}$, are the deterministic restriction operators defined as [17, 24, 140]

$$
\left\{\begin{array}{l}
\mathbf{u}_{r}^{s, j} \\
\mathbf{u}_{c}^{s, j}
\end{array}\right\}=\left\{\begin{array}{l}
\mathbf{R}_{s, j}^{r} \\
\mathbf{R}_{s, j}^{c}
\end{array}\right\} \mathbf{u}_{\Gamma}^{s, j}
$$

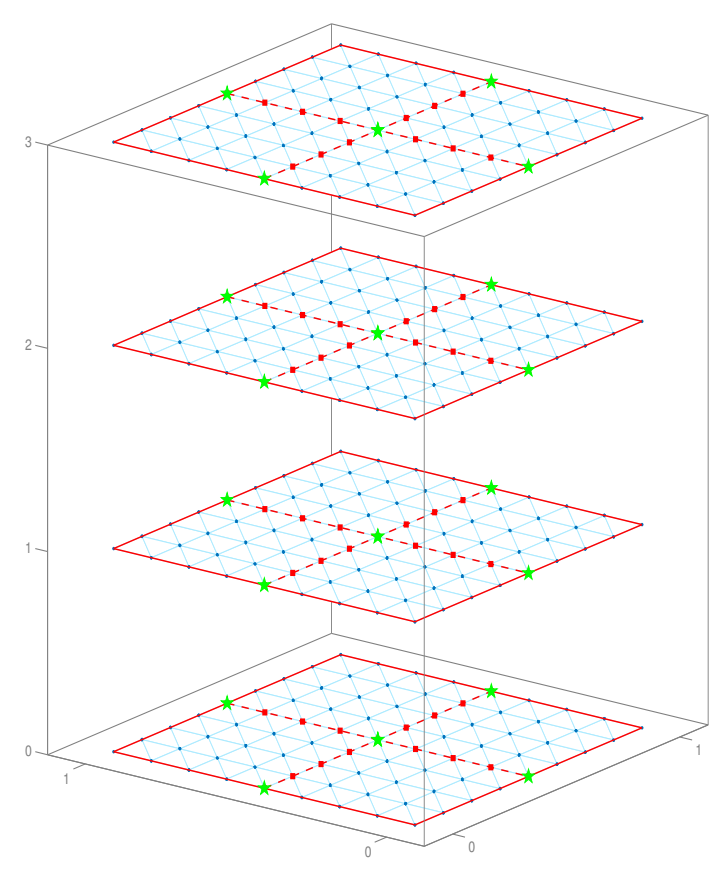

Figure 3.3: First four PCE coefficients of the solution with two-level mesh decomposition showing interior(i), aremaining(r) and $\star$ corner(c) nodes.

A subdomain-level stochastic Neumann problem is solved in the implementation of the ex- 
tended Neumann-Neumann preconditioner is defined as [17, 24, 140]:

$$
\left[\begin{array}{ccc}
A_{i i}^{s} & A_{i r}^{s} & A_{i c}^{s} \\
A_{r i}^{s} & A_{r r}^{s} & A_{r c}^{s} \\
A_{c i}^{s} & A_{c r}^{s} & A_{c c}^{s}
\end{array}\right]\left\{\begin{array}{l}
x_{i}^{s} \\
u_{r}^{s} \\
u_{c}^{s}
\end{array}\right\}=\left\{\begin{array}{c}
\mathbf{0} \\
f_{r}^{s} \\
f_{c}^{s}
\end{array}\right\},
$$

where $f_{r}^{s}$ and $f_{c}^{s}$ are obtained by $[17,24,140]$,

$$
\left\{\begin{array}{l}
f_{r}^{s} \\
f_{c}^{s}
\end{array}\right\}=\left\{\begin{array}{l}
R_{s}^{r} \\
R_{s}^{c}
\end{array}\right\} \mathbf{r}_{\Gamma}^{\mathbf{s}} .
$$

The continuity condition is satisfied on the global corner nodes by performing a partial assembly of Eq. (3.24). Eliminating the local interior $x_{i}^{s}$ and remaining $u_{r}^{s}$ variables from resulting equation leads to the following symmetric positive definite global coarse problem (see [24] and references therein for further details).

$$
F_{c c} u_{c}=d_{c}
$$

where the global coarse operator $F_{c c}$, being the Schur complement of extended Schur complements $S_{\alpha \beta}^{s}$, and the corresponding right hand side vector are given by [17,24]

$$
\begin{aligned}
F_{c c} & =\sum_{s=1}^{n_{s}} B_{c}^{s T}\left(S_{c c}^{s}-S_{c r}^{s}\left[S_{r r}^{s}\right]^{-1} S_{r c}^{s}\right) B_{c}^{s} \\
d_{c} & =\sum_{s=1}^{n_{s}} B_{c}^{s T}\left(F_{c}^{s}-S_{c r}^{s}\left[S_{r r}^{s}\right]^{-1} F_{r}^{s}\right) .
\end{aligned}
$$

Each $S_{\alpha \beta}^{s}$ is defined using blocks of the subdomain-level stochastic coefficient matrix $A_{\alpha \beta}^{s}$, with subscripts $\alpha$ and $\beta$ are for index $r$ and $c$,

$$
S_{\alpha \beta}^{s}=A_{\alpha \beta}^{s}-A_{\alpha i}^{s}\left[A_{i i}^{s}\right]^{-1} A_{i \beta}^{s}
$$


$B_{c}^{s}$ is the Boolean operator that maps global corner node coefficients $u_{c}$ into local corner node coefficients $u_{c}^{s}$. It is obtained as [17]

$$
B_{c}^{s}=\operatorname{blockdiagonal}\left(\mathbf{B}_{c}^{s, 0}, \ldots, \mathbf{B}_{c}^{s, P_{u}}\right)
$$

where the deterministic restriction operator $\mathbf{B}_{c}^{s, j}$ with $j=\left\{0,1, \ldots, P_{u}\right\}$, maps the global corner node solution vector into local corner node output as:

$$
\mathbf{u}_{c}^{s, j}=\mathbf{B}_{c}^{s, j} \mathbf{u}_{c}^{j}
$$

Solving the global coarse problem in Eq. (3.26), we obtain the corner node coefficients $u_{c}$. The remaining interface solution vector is computed by solving one fine problem given in Eq. (3.32) on each subdomain using the corner node output as [17,24].

$$
S_{r r}^{s} u_{r}^{s}=F_{r}^{s}-S_{r c}^{s} u_{c}^{s}
$$

The local interface output is used to obtain global interface unknowns using Eq. (3.33) as [24]:

$$
u_{\Gamma}=\sum_{s=1}^{n_{s}} R_{s}^{T} D_{s}\left(R_{s}^{r T} u_{r}^{s}+R_{s}^{c T} u_{c}^{s}\right)
$$

where $D_{s}=$ blockdiagonal $\left(\mathbf{D}_{s, 0}, \ldots, \mathbf{D}_{s, P_{u}}\right)$, obtained by using a diagonal scaling matrices $\mathbf{D}_{s, j}$ (with $j=1,2, \ldots, P_{u}$ ), defined by

$$
\sum_{s=1}^{n_{s}} \mathbf{R}_{s, j}^{T} \mathbf{D}_{s, j} \mathbf{R}_{s, j}=\mathbf{I}
$$

Performing algebraic manipulation using Eq. (3.26), Eq. (3.32) and Eq. (3.33), the two-level NNC preconditioner is written as (refer [24] for further details),

$$
M_{N N C}^{-1}=\sum_{s=1}^{n_{s}} R_{s}^{T} D_{s}\left(R_{s}^{r T}\left[S_{r r}^{s}\right]^{-1} R_{s}^{r}\right) D_{s} R_{s}+R_{0}^{T}\left[F_{c c}\right]^{-1} R_{0}
$$


$R_{0}$ acts as a mapping operator between fine and coarse grid [17,24],

$$
R_{0}=\sum_{s=1}^{n_{s}} B_{c}^{s T}\left(R_{s}^{c}-S_{c r}^{s}\left[S_{r r}^{s}\right]^{-1} R_{s}^{r}\right) D_{s} R_{s} .
$$

From Eq. (3.35) it can be noted that the stochastic BDDC/NNC preconditioner consists of two levels. First, the fine level, which consists of solving one local fine problem, $\left[S_{r r}^{s}\right]^{-1}$ on each of the subdomains. Second, the coarse level, which consists of solving a global coarse problem, $F_{c c}^{-1}$ for each of PCGM iteration. The coarse level provides mechanism for propagating the information about the error across subdomains, thus accelerate the convergence of the iterative solver by rapidly reducing the components of the error $[17,125,128,140,200]$.

\subsubsection{Non-Overlapping Domain Decomposition - the Dual-Primal Approach}

This section is dedicated to the review of the probabilistic version of dual-primal domain decomposition methods for uncertainty quantification following the references [18, 24, 148]. We will focus on dual-primal finite element tearing and interconnect (FETI-DP) technique [131] to solve the linear system of equations formulated using SSFEM. The main differences between the dual-primal and the primal approaches are discussed next. In the FETI-DP, the coarse problem is embedded into the dual-operator as opposed to BDDC/NNC in which the coarse problem is incorporated through the application of the preconditioner. In the primal approach, the Schur complement system (primal interface problem) is solved using the two-level preconditioner. In dual-primal approach, the Lagrange multipliers are introduced to form the dual-interface system, which is later solved iteratively using PCGM with one-level preconditioners [17, 18, 24, 148].

To employ FETI-DP procedure, the spatial domain $D$ is partitioned into $n_{s}$ non-overlapping subdomains with two-level decomposition as shown in Fig. 3.3. The subdomain-level solution vector is divided into interior $(i)$, corner $(c)$ and remaining $(r)$ nodes. Using PC expansion given in Eq. (3.5) for the random system parameters and solution process and performing Galerkin 
projection onto the subdomain-level equilibrium equation, the following stochastic system is constructed [18,24],

$$
\left[\begin{array}{ccc}
A_{i i}^{s} & A_{i r}^{s} & A_{i c}^{s} \\
A_{r i}^{s} & A_{r r}^{s} & A_{r c}^{s} \\
A_{c i}^{s} & A_{c r}^{s} & A_{c c}^{s}
\end{array}\right]\left\{\begin{array}{l}
u_{i}^{s} \\
u_{r}^{s} \\
u_{c}^{s}
\end{array}\right\}=\left\{\begin{array}{l}
f_{i}^{s} \\
f_{r}^{s} \\
f_{c}^{s}
\end{array}\right\},
$$

where

$$
\begin{gathered}
{\left[A_{\alpha \beta}^{s}\right]_{j k}=\sum_{i=0}^{P_{\mathrm{A}}}\left\langle\Psi_{i} \Psi_{j} \Psi_{k}\right\rangle\left[\overline{\mathbf{A}}_{\alpha \beta, i}^{s}\right]} \\
{\left[f_{\alpha}^{s}\right]_{k}=\left\langle\Psi_{k} \mathbf{f}_{\alpha}^{s}\right\rangle} \\
u_{\alpha}^{s}=\left(\overline{\mathbf{u}}_{\alpha, 0}^{s}, \ldots, \overline{\mathbf{u}}_{\alpha, P_{u}}^{s}\right)^{T}
\end{gathered}
$$

and the subscripts $\alpha$ and $\beta$ relate to $i, r$ and $c$.

The Lagrange multipliers $\{\Lambda\}$ are used to enforce weak compatibility constraint on the remaining node PCE coefficients and then, performing a global assembly the following system is obtained [18, 24],

$$
\left[\begin{array}{cccc}
A_{i i}^{s} & A_{i r}^{s} & A_{i c}^{s} B_{c}^{s} & 0 \\
A_{r i}^{s} & A_{r r}^{s} & A_{r c}^{s} B_{c}^{s} & B_{r}^{s T} \\
\sum_{s=1}^{n_{s}} B_{c}^{s T} A_{c i}^{s} & \sum_{s=1}^{n_{s}} B_{c}^{s T} A_{c r}^{s} & \sum_{s=1}^{n_{s}} B_{c}^{s T} A_{c c}^{s} B_{c}^{s} & 0 \\
0 & \sum_{s=1}^{n_{s}} B_{r}^{s} & 0 & 0
\end{array}\right]\left\{\begin{array}{c}
u_{i}^{s} \\
u_{r}^{s} \\
u_{c} \\
\Lambda
\end{array}\right\}=\left\{\begin{array}{c}
f_{i}^{s} \\
f_{r}^{s} \\
\sum_{s=1}^{n_{s}} B_{c}^{s T} f_{c}^{s} \\
0
\end{array}\right\} .
$$

Here $\Lambda$ is a vector of deterministic coefficients defined as [18,24],

$$
\Lambda=\left[\bar{\lambda}_{0}, \ldots, \bar{\lambda}_{N_{r}}\right]^{T}
$$

where $N_{r}$ is the total number of remaining interface nodes and the jump operator $B_{r}^{s}$ is defined 
as [18, 24],

$$
\sum_{s=1}^{n_{s}} B_{r}^{s} u_{r}^{s}=0
$$

Performing Gaussian elimination in Eq. (3.41), the Lagrange multiplier system of the polynomial chaos coefficients is obtained as (refer to [18,24] for details),

$$
\left[F_{r r}+F_{r c}\left[F_{c c}\right]^{-1} F_{c r}\right] \Lambda=\left(d_{r}-F_{r c}\left[F_{c c}\right]^{-1} d_{c}\right)
$$

where $\left[F_{c c}\right]^{-1}$ is the coarse operator, being the Schur complement of Schur complement matrices $S_{\alpha \beta}^{s}$ as follows

$$
F_{c c}=\sum_{s=1}^{n_{s}} B_{c}^{s T}\left(S_{c c}^{s}-S_{c r}^{s}\left[S_{r r}^{s}\right]^{-1} S_{r c}^{s}\right) B_{c}^{s} .
$$

The remaining terms from Eq. (3.44) are also given in expanded form as follows [18, 24]:

$$
\begin{aligned}
F_{r r} & =\sum_{s=1}^{n_{s}} B_{r}^{s}\left[S_{r r}^{s}\right]^{-1} B_{r}^{s T}, \\
F_{r c} & =\sum_{s=1}^{n_{s}} B_{r}^{s}\left[S_{r r}^{s}\right]^{-1} S_{r c}^{s} B_{c}^{s}, \\
F_{c r} & =\sum_{s=1}^{n_{s}} B_{c}^{s T} S_{c r}^{s}\left[S_{r r}^{s}\right]^{-1} B_{r}^{s T}, \\
d_{c} & =\sum_{s=1}^{n_{s}} B_{c}^{s T}\left(G_{c}^{s}-S_{c r}^{s}\left[S_{r r}^{s}\right]^{-1} G_{r}^{s}\right), \\
d_{r} & =\sum_{s=1}^{n_{s}} B_{r}^{s}\left[S_{r r}^{s}\right]^{-1} G_{r}^{s},
\end{aligned}
$$

where

$$
\begin{aligned}
S_{\alpha \beta}^{s} & =A_{\alpha \beta}^{s}-A_{\alpha i}^{s}\left[A_{i i}^{s}\right]^{-1} A_{i \beta}^{s}, \\
G_{\alpha}^{s} & =F_{\alpha}^{s}-A_{\alpha i}^{s}\left[A_{i i}^{s}\right]^{-1} F_{i}^{s} .
\end{aligned}
$$

For elliptic stochastic PDEs, the extended Lagrange multiplier system equipped with the 
coarse operator $F_{c c}^{-1}$ in Eq. (3.44) is symmetric and positive definite [18, 131, 150]. Hence, the PCGM with one-level preconditioner can be employed to solve the system. Note that for each of the PCGM iterations, the continuity of the PCE coefficients is strictly satisfied only at the corner nodes. The continuity across the entire interface PCE coefficients is strictly satisfied only at the convergence of PCGM iterations [18, 148].

The preconditioned extended Lagrange multiplier system is given by [18, 24]

$$
M_{D}^{-1}\left[F_{r r}+F_{r c}\left[F_{c c}\right]^{-1} F_{c r}\right] \Lambda=M_{D}^{-1}\left(d_{r}-F_{r c}\left[F_{c c}\right]^{-1} d_{c}\right)
$$

Consequently Eq. (3.53) is solved iteratively using the extended Dirichlet preconditioner as [18, 24]:

$$
M_{D}^{-1}=\sum_{s=1}^{n_{s}} B_{r}^{s} D_{r}^{s}\left[S_{r r}^{s}\right] D_{r}^{s} B_{r}^{s T},
$$

where the weighting matrix $D_{r}^{s}$ is defined as

$$
D_{r}^{s}=\operatorname{blockdiagonal}\left(\mathbf{D}_{r}^{s, 0}, \ldots, \mathbf{D}_{r}^{s, P_{u}}\right)
$$

The diagonal averaging matrices $\mathbf{D}_{r}^{s, j}$ in Eq. (3.55) where $j=0,1, \ldots, P_{u}$, are obtained as follows [131, 148],

$$
\sum_{s=1}^{n_{s}} \mathbf{K}_{s, j}^{T} \mathbf{D}_{r}^{s, j} \mathbf{K}_{s, j}=\mathbf{I}
$$

where $\mathbf{K}_{s, j}$ is a scatter or gather operator between global and local components of the deterministic remaining solution vectors as [131],

$$
\mathbf{u}_{r}^{s, j}=\mathbf{K}_{s, j} \mathbf{u}_{r}^{j}, \quad \mathbf{u}_{r}^{j}=\mathbf{K}_{s, j}^{T} \mathbf{u}_{r}^{s, j}
$$

Once the Lagrange multipliers are available, they are used to obtained PCE coefficients of 
the solution process associated with corner nodes as [18, 24]

$$
\begin{aligned}
F_{c c} u_{c} & =d_{c}+F_{c r} \Lambda, \\
u_{c}^{s} & =B_{c}^{s} u_{c} .
\end{aligned}
$$

Finally, the subdomain-level solution coefficients associated with interior $\left(u_{i}^{s}\right)$ and remaining $\left(u_{r}^{s}\right)$ nodes are obtained by solving the following problem on each subdomain as [18,24]

$$
\left[\begin{array}{cc}
A_{i i}^{s} & A_{i r}^{s} \\
A_{r i}^{s} & A_{r r}^{s}
\end{array}\right]\left\{\begin{array}{l}
u_{i}^{s} \\
u_{r}^{s}
\end{array}\right\}=\left\{\begin{array}{c}
f_{i}^{s}-A_{i c}^{s} u_{c}^{s} \\
f_{r}^{s}-A_{r c}^{s} u_{c}^{s}-B_{r}^{s T} \Lambda
\end{array}\right\} .
$$

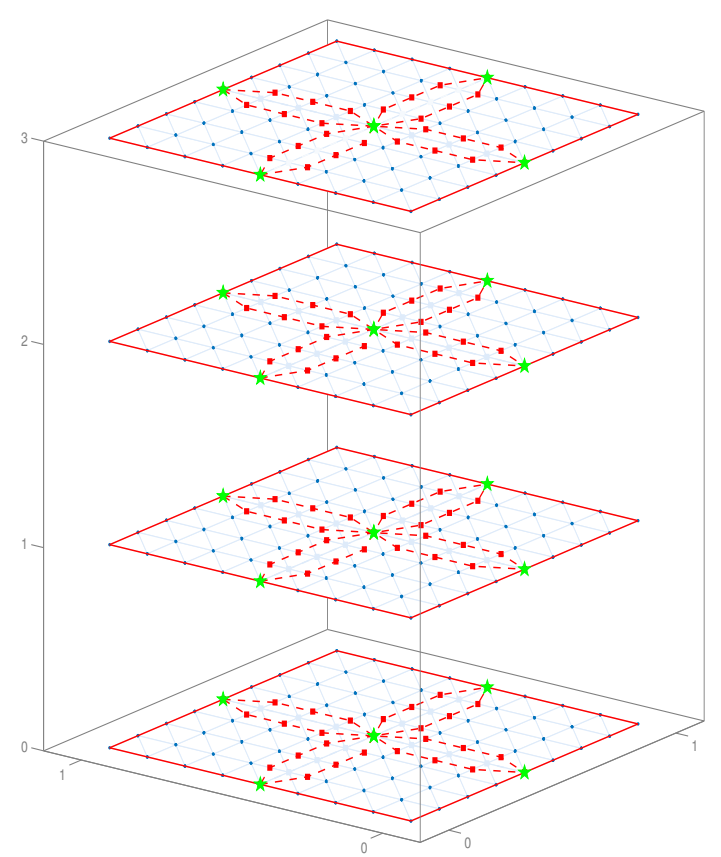

Figure 3.4: First four PCE coefficients of the solution showing FETI-DP mesh decomposition with interior(i), aremaining(r) and $\star$ corner(c) nodes.

For further details on mathematical formulation and theoretical comparison between BDDC and FETI-DP type approaches for stochastic PDEs, we refer to [18,24] and references therein. 
For illustrative purposes, the first four PCE coefficients of the solution process associated with interior, corner and remaining nodes along with the FETI-DP based mesh decomposition is shown in Fig. 3.4.

\subsection{Parallel Implementation of Stochastic Domain Decompo- sition Solvers}

The probabilistic versions of the primal and dual-primal domain decomposition solvers formulated in previous sections are implemented in parallel using the PCGM algorithm [24] with MPI and PETSc libraries [31]. Sec. 3.2.1 provides an overview of PCGM algorithm with emphasis on the key steps. Sec. 3.2.2 is devoted to illustrating the efficient implementation procedure of BDDC/NNC solver using PETSc objects and routines. Sec. 3.2.3 is dedicated to demonstrating the FETI-DP solver implementation using PETSc.

\subsubsection{Parallel PCGM Algorithm}

The parallel implementation of PCGM is depicted in Algorithm 1 [18,24]. For illustration, the procedure is shown only for the preconditioned extended Schur complement system given in Eq. (3.18). However, the same algorithm is employed for the preconditioned extended Lagrange multiplier system formulated in Eq. (3.53).

The computationally intensive steps 7 and 13 in the Algorithm 1 are highlighted (in blue). Both steps warrant careful implementation to efficiently handle large-scale simulations. The first essential step is to calculate the matrix-vector product and the second is to compute the effect of preconditioner on the residual. In the case of non-overlapping domain decomposition method, these procedures can be performed on each subdomain in parallel. In practice, the explicit construction of preconditioner $\left(M^{-1}\right)$ and Schur complement matrix $(S)$ is not needed. Instead, Parallel Preconditioning (PP) procedure and Parallel Mat-Vec Product (PMVP) procedures are used. With the increase in problem size, these two steps become crucial to the 


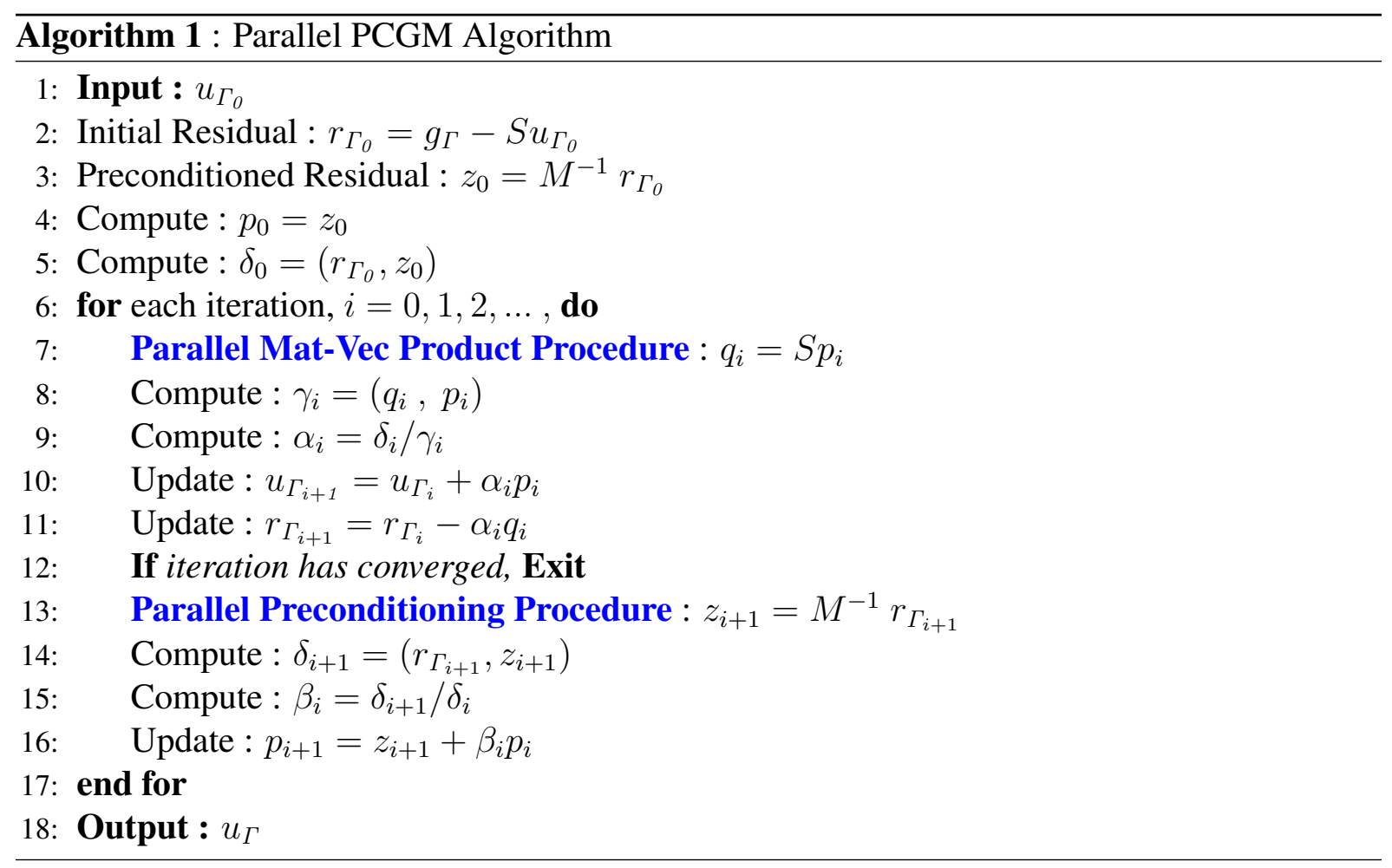

performance of the solvers concerning memory and floating-point operations. Efficient implementation procedure of the Algorithm 1 focussing on steps 7 and 13 for both BDDC/NNC and FETI-DP solvers is discussed in the next sections respectively.

\subsubsection{Implementation of BDDC/NNC-PCGM Algorithm Using PETSc}

The following section is dedicated to the efficient implementation of Algorithm 1 using twolevel BDDC/NNC preconditioner formulated in Sec. 3.1.1. The key steps of the solver (highlighted in blue) are the parallel matrix-vector product procedure and parallel preconditioning procedure. Implementation of both these steps demands the following: (a) assembly of the blocks of subdomain-level (local) stochastic finite element matrices, (b) computation of several of the local matrix-vector products and (c) solution of multiple subdomain-level linear systems of equations (local problems). To implement the above procedures, we deal only with the subdomain-level operations. Hence, the memory required to assemble and solve the local problem restricts the total size of the (global) problem we can tackle using the given machine 
architecture. By optimally using the available memory, we can tackle much larger sizes of local problems. This can be achieved using sparse PETSc data structures [31] to assemble and store matrices and vectors. Sparse PETSc routines [31] are used whenever applicable. Sparse PETSc linear solvers [31] are used for the local problems.

To create the sparse PETSc matrices (local), the MatCreateSeqAIJ routine is used. This PETSc object stores the sparse matrix in compressed row format [31]. For illustration, the stochastic finite element matrix assembly procedure is outlined in Algorithm 2 for one block of the subdomain-level stochastic matrix. Algorithm 2 corresponds to Eq. (3.8) in which the stochastic finite element matrix blocks are filled using deterministic finite element matrix blocks. In this procedure, efficient usage of available memory is achieved while making use of Mat, the sparse data structure from PETSc. Note that the blocks of deterministic matrices are destroyed after the stochastic assembly procedure is complete. This is convenient for the reuse of the available memory in order to efficiently handle the cases of large number of random variables.

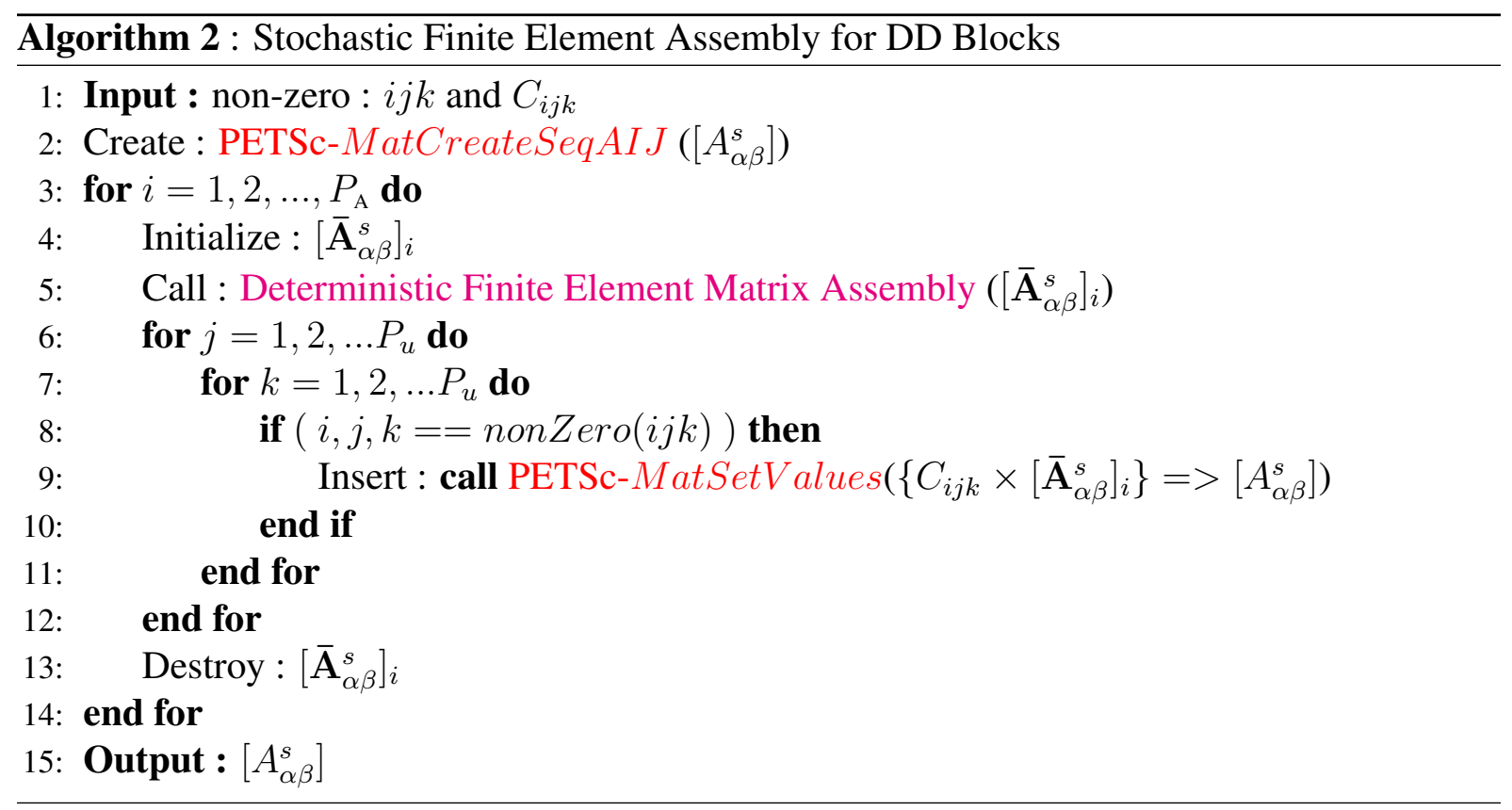

In the current implementation of the stochastic assembly procedure, the deterministic matrix assembly is performed using the element-level FEniCS assembly routines [32] and validated 
by an in-house assembly code, initially implemented by Khalil [21, 178] and later extended by the author [19]. Direct use of the FEniCS routines in the proposed algorithm can simplify the stochastic system matrix assembly procedure for a wide variety of PDEs and offers the ability to tackle numerous practical applications [32].

The sparse PETSc objects as in Mat and Vec employed here reduce the memory required to assemble and store the local blocks of the matrices and vectors. As they are available in PETSc format, one could make use of various sparse linear Krylov subspace $(K S P)$ based solvers available in PETSc to solve the multiple subdomain-level problems. Furthermore, one could employ the other sparse linear algebra routines from PETSc for commonly occurring algebraic operations in BDDC/NNC-PCGM implementation (for instance, local matrix-vector products by using MatMult, local dot-products by employing VecDot, matrix-transpose-vector products by using MatMultTranspose, vector-multiplications by utilizing VecAXPY, vector-norm by using VecNorm). These routines will assist in efficiently performing the repeatedly occurring algebraic operations for DDM based PCGM solvers, hence optimizing the memory usage and floating-point operation count throughout the implementation. An increase in stochastic dimensionality enhances the sparsity of the stochastic matrices [1,90] highlighting the benefit of scalable sparse objects, routines and solvers in tackling much larger linear systems. Further details of the implementation procedure for the major steps of BDDC/NNC-PCGM solvers using PETSc is discussed in next sections.

\section{Parallel Matrix-Vector Product Procedure for BDDC/NNC-PCGM}

The parallel matrix-vector product (PMVP) procedure for the extended Schur compliment system shown in Eq. (3.18) is expanded below,

$$
\begin{aligned}
q_{i} & =S p_{i}, \\
& =\sum_{s=1}^{n_{s}} R_{s}^{T}\left[A_{\Gamma \Gamma}^{s}-A_{\Gamma I}^{s}\left[A_{I I}^{s}\right]^{-1} A_{I \Gamma}^{s}\right] R_{s} p_{i} .
\end{aligned}
$$




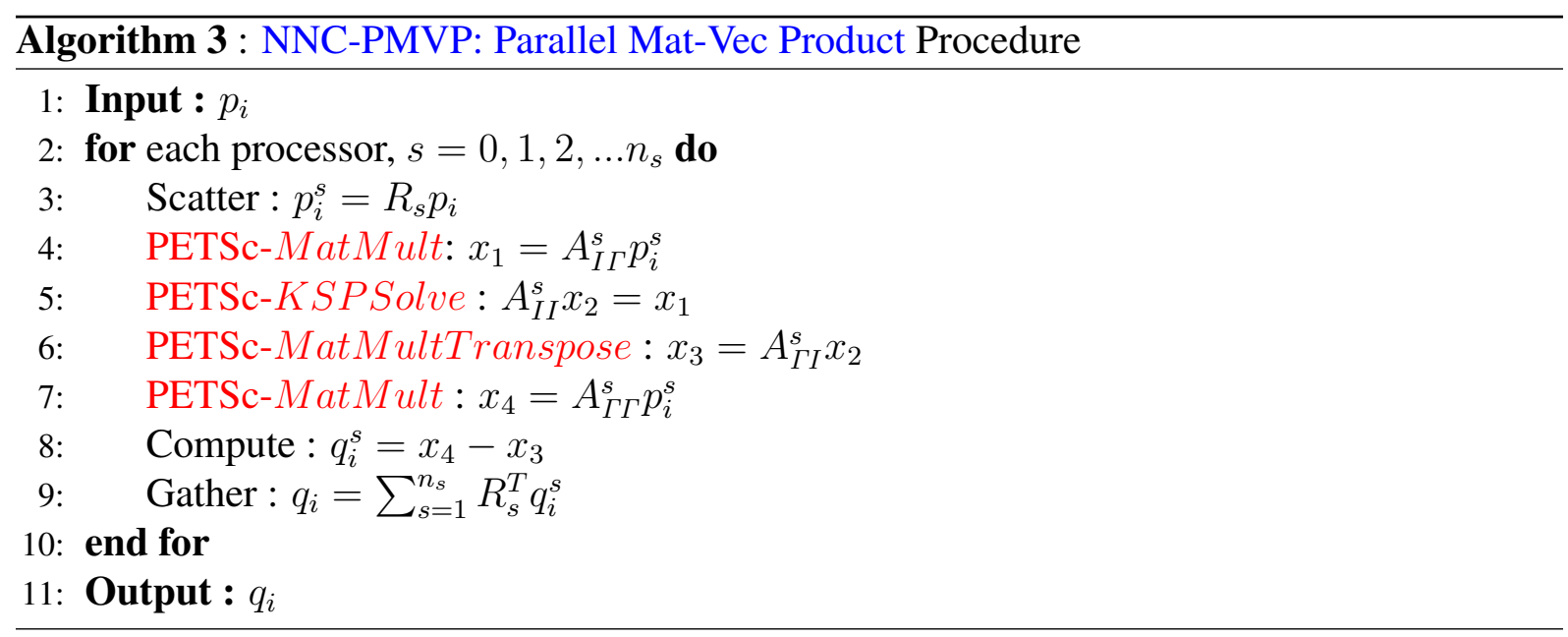

First, the blocks of subdomain-level stochastic matrices and vectors $\left(A_{I I}^{s}, A_{I \Gamma}^{s}, f_{I}^{s}\right.$ etc.) are directly assembled in sparse PETSc format using the Algorithm 2. Each of these local blocks are highly sparse and hence the use of the sparse objects optimizes the memory required to store these blocks. Subsequently the PETSc-MatMult routines are employed to carry out three sparse matrix-vector products needed for the procedure. The PETSc-KSPCG, a sparse PCGM solver with block-Jacobi (PCBJACOBI) preconditioner [31] is employed to solve the subdomain-level interior problem. The PMVP procedure for BDDC/NNC-PCGM is detailed in Algorithm 3. Note that all the highlighted (in red) steps are implemented using PETSc routines.

PETSc also provides the sparse direct solvers like $M U M P S$, but we decided to use sparse iterative solvers (in this case, $K S P C G$ ) mainly for the following reasons: (a) for our applications, the direct solver requires more memory compared to iterative solvers; and (b) the iterative $K S P C G$ solvers used here can be further accelerated using more efficient preconditioners. The preconditioners can be built into PETSc or applied externally to PETSc. In the current implementation, we are applying PETSc block Jacobi preconditioner [31]. In the future implementations of the solvers, we will use more efficient external preconditioners such as Kronecker product preconditioner [154] and hierarchical Schur complement preconditioner [155] to further accelerate the convergence of the subdomain-level interior problems. 


\section{Two-Level Parallel Preconditioning Procedure for BDDC/NNC-PCGM}

As specified in Algorithm 1, for each of the main PCGM iteration on extended Schur complement system given by Eq. (3.18), we perform the parallel preconditioning effect procedure. In the case of two-level BDDC/NNC preconditioner (given by Eq. (3.35)), it is accomplished using Algorithm 4. The procedure consists of three main tasks (highlighted in blue): (a) Local Neumann solve (LNS) procedure (step 6 and 16), (b) local matrix-vector product (LMVP) procedure (step 7 and 14) and (c) global coarse problem (GCP) (step 11). Each of these tasks is accomplished separately by using three different PETSc-based algorithms given below.
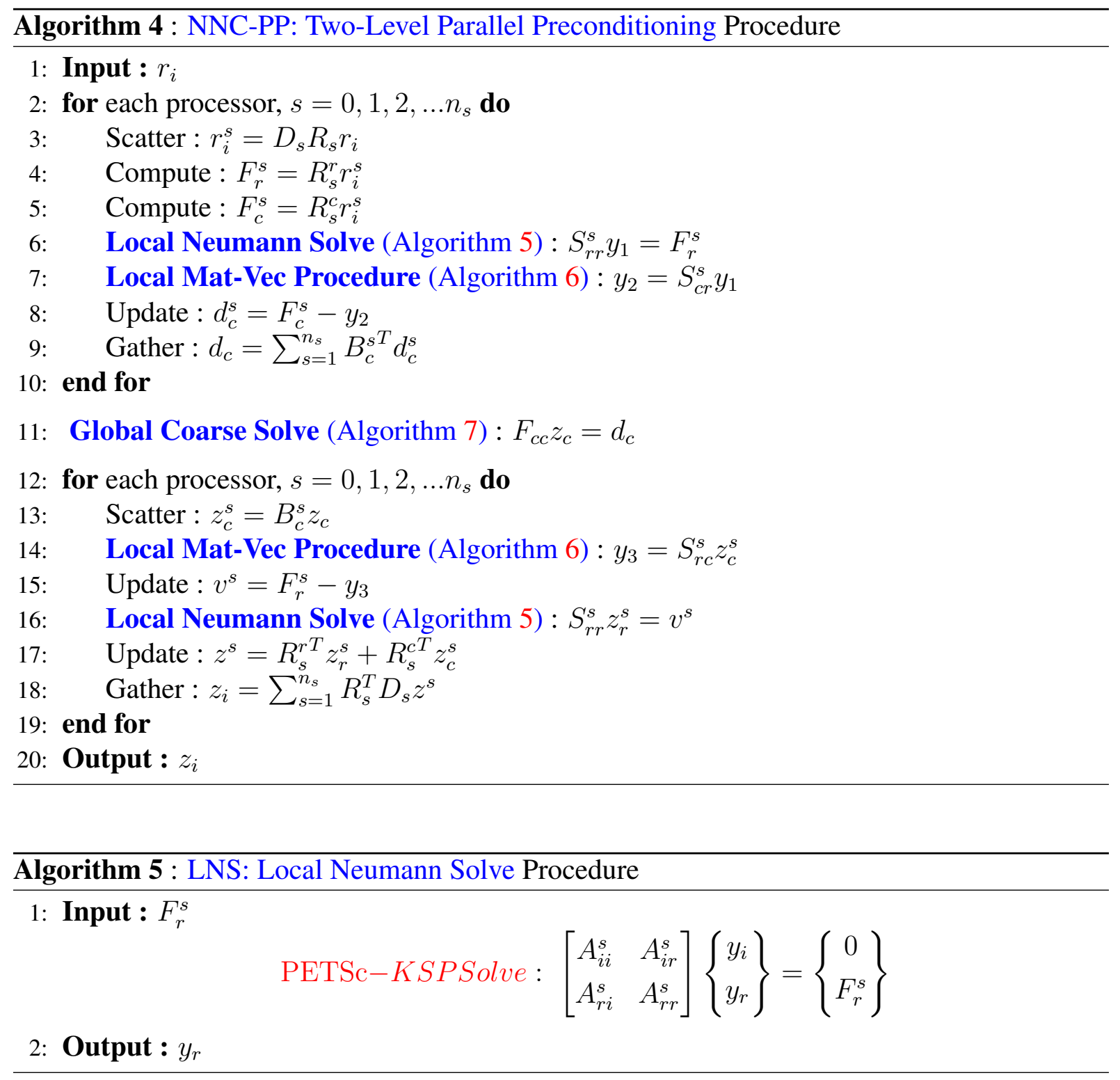
Algorithm 5 provides the local Neumann solve (LNS) procedure using PETSc-KSPCG to solve the subdomain-level Schur complement matrix $\left(S_{r r}^{s}\right)$ given by Eq. (3.29). The LNS procedure is utilized twice for each application of the preconditioner as outlined in Algorithm 4. Next, the local matrix-vector product (LMVP) procedure given in eq. (3.62) is performed using Algorithm 6. Here, the PETSc-MatMult routines are employed to carry out the three sparse matrix-vector product operations and the PETSc-KSPCG solver, equipped with block-Jacobi preconditioner, is used to solve the local interior problem as in

$$
\begin{aligned}
y_{\text {out }} & =S_{\alpha \beta}^{s} y_{\text {in }}, \\
& =\left[A_{\alpha \beta}^{s}-A_{\alpha i}^{s}\left[A_{i i}^{s}\right]^{-1} A_{i \beta}^{s}\right] y_{i n} .
\end{aligned}
$$

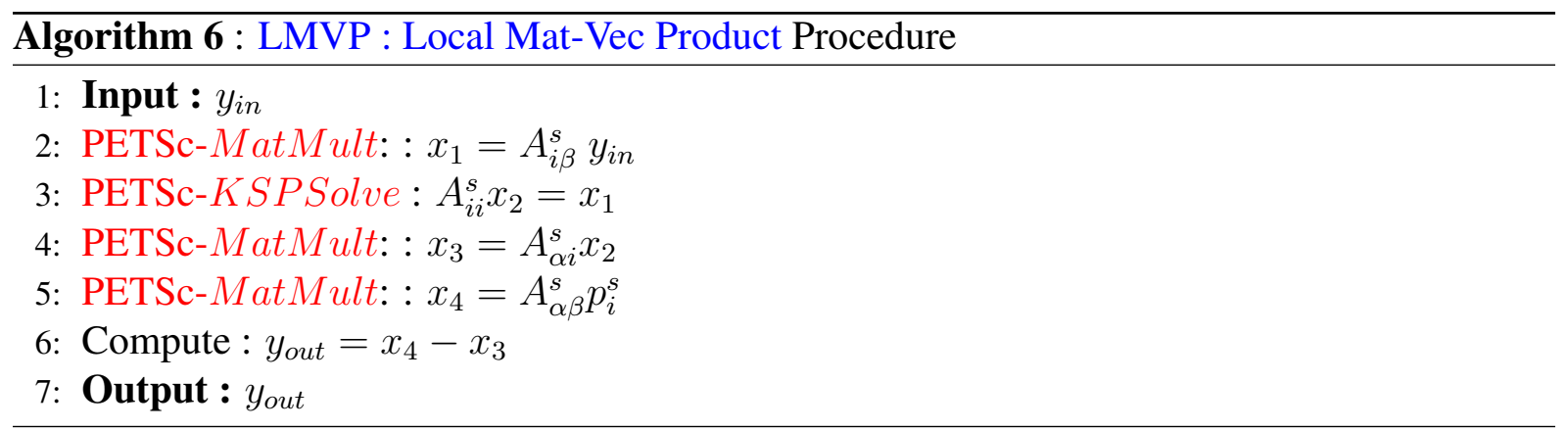

The application of BDDC/NNC preconditioner also requires the solution of the global coarse problem (GCP) defined by Eq. (3.26) for each of the main PCGM iteration (see Algorithm 4). The preconditioned global coarse problem is given by

$$
M^{-1} F_{c c} u_{c}=M^{-1} d_{c}
$$

The GCP is solved using another parallel PCGM procedure (coarse-PCGM) with a one-level lumped preconditioner as outlined in Algorithm 7. The coarse-PCGM algorithm contains two major steps 6 and 12 (highlighted in blue): (a) parallel Schur-matrix-vector product (Sc-PMVP) 


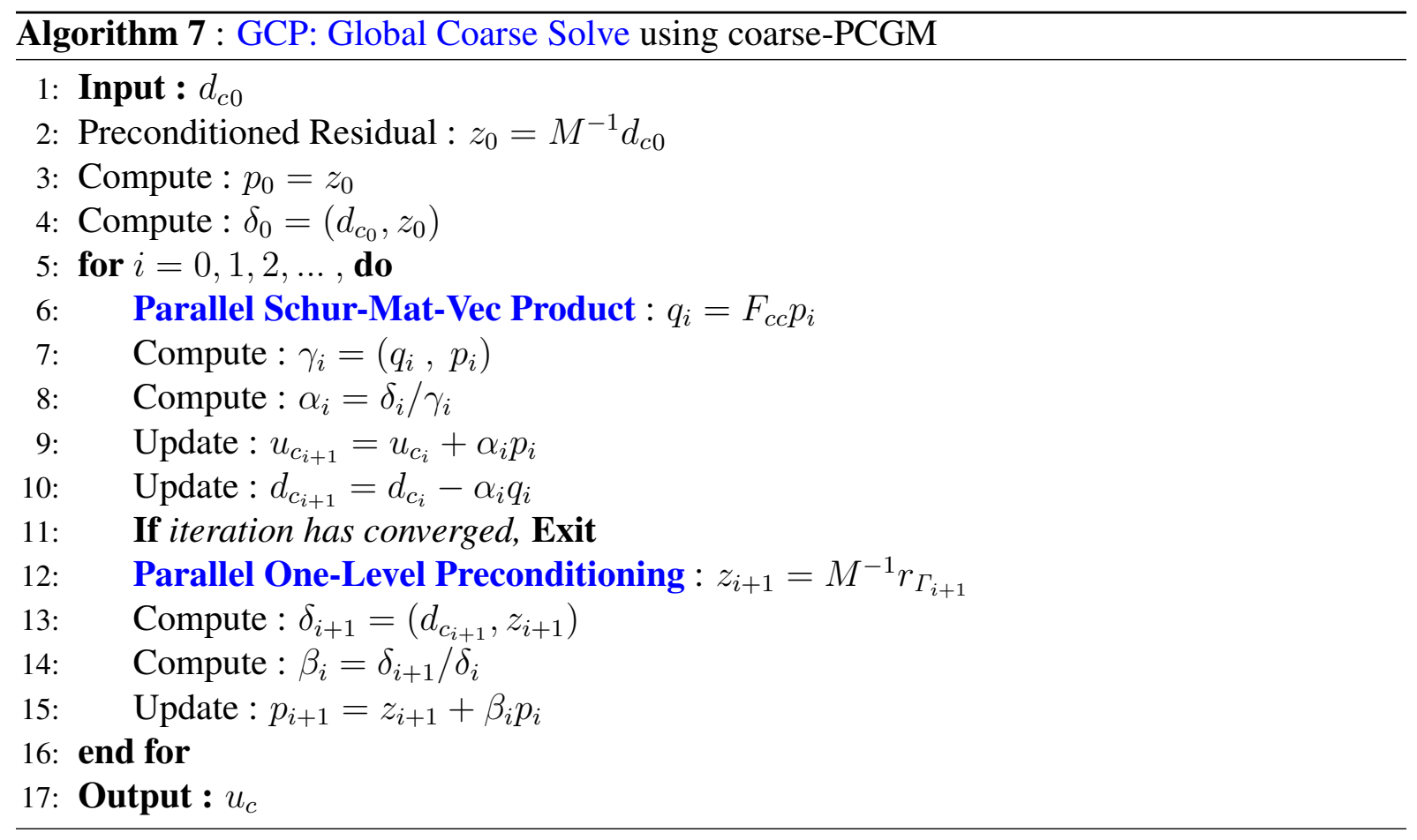

procedure performed using Algorithm 8 and (b) parallel one-level lumped preconditioning (LPP) procedure performed using Algorithm 9. The Schur-matrix-vector product procedure is the product of a Schur compliment matrix with a vector as shown in eq. (3.64) below,

$$
\begin{aligned}
q_{i} & =F_{c c} p_{i} \\
& =\sum_{s=1}^{n_{s}} B_{c}^{s T}\left[S_{c c}^{s}-S_{c r}^{s}\left[S_{r r}^{s}\right]^{-1} S_{r c}^{s}\right] B_{c}^{s} p_{i} .
\end{aligned}
$$

The Sc-PMVP procedure is implemented using Algorithm 8 which employs the three LMVP procedures given in Algorithm 6 and the local Neumann solve procedure given in Algorithm 5.

The effect of one-level lumped preconditioner given by ( Eq. (3.65)) is performed in parallel using Algorithm 9. This procedure requires solving one local problem, which is accomplished 


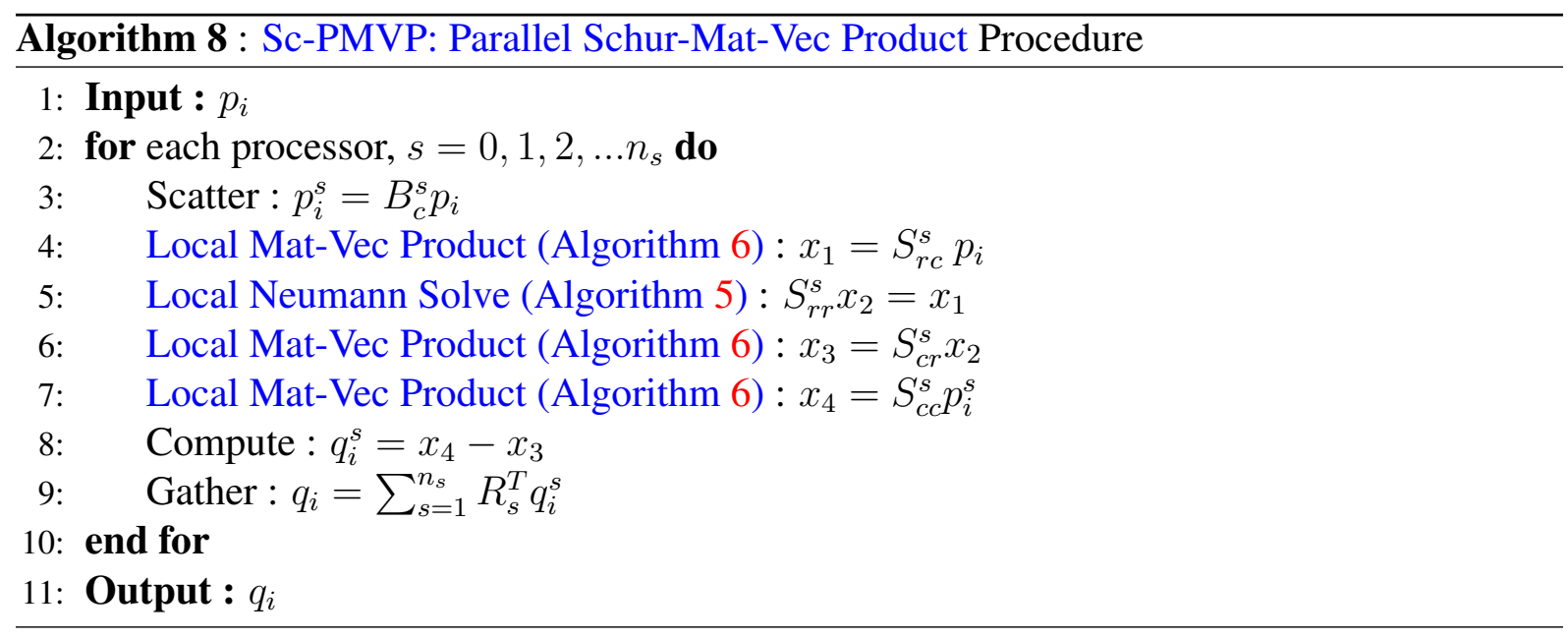

using PETSc-KSPCG solver with the block-Jacobi preconditioner as in

$$
\begin{aligned}
z_{i} & =M^{-1} r_{i}, \\
& =\sum_{s=1}^{n_{s}} R_{s}^{T}\left[M_{s}\right]^{-1} R_{s} r_{i} .
\end{aligned}
$$

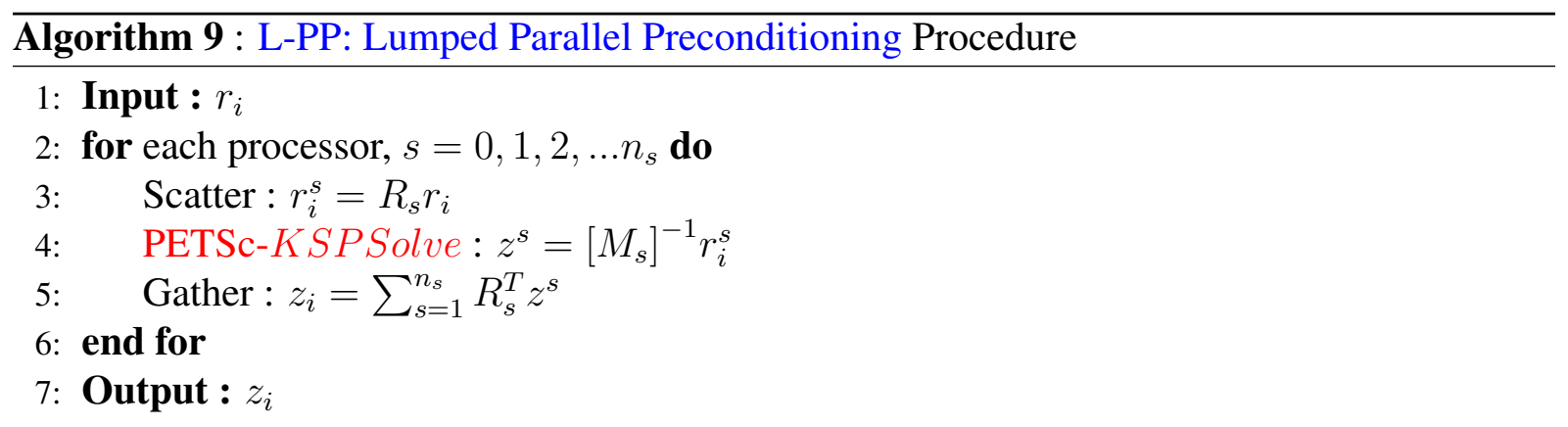

The flowchart detailing the implementation of the BDDC/NNC-PCGM solver is given in Fig. 3.5. To tackle the preconditioned extended Schur complement system using BDDC/NNCPCGM solver, we have to perform (a) one NNC-PMVP procedure, (b) one L-PP procedure, (c) two LNS procedures and (d) two LMPV procedures. These procedures are accomplished using the sparse PETSc objects, routines and solvers as outlined previously. Note that Sc-PMVP uses Algorithm 5 and Algorithm 6.

PETSc routines are only used for the subdomain-level sparse matrix-vector operations (i.e. local system solve and local matrix-vector products etc). PETSc is not used for any parallel 


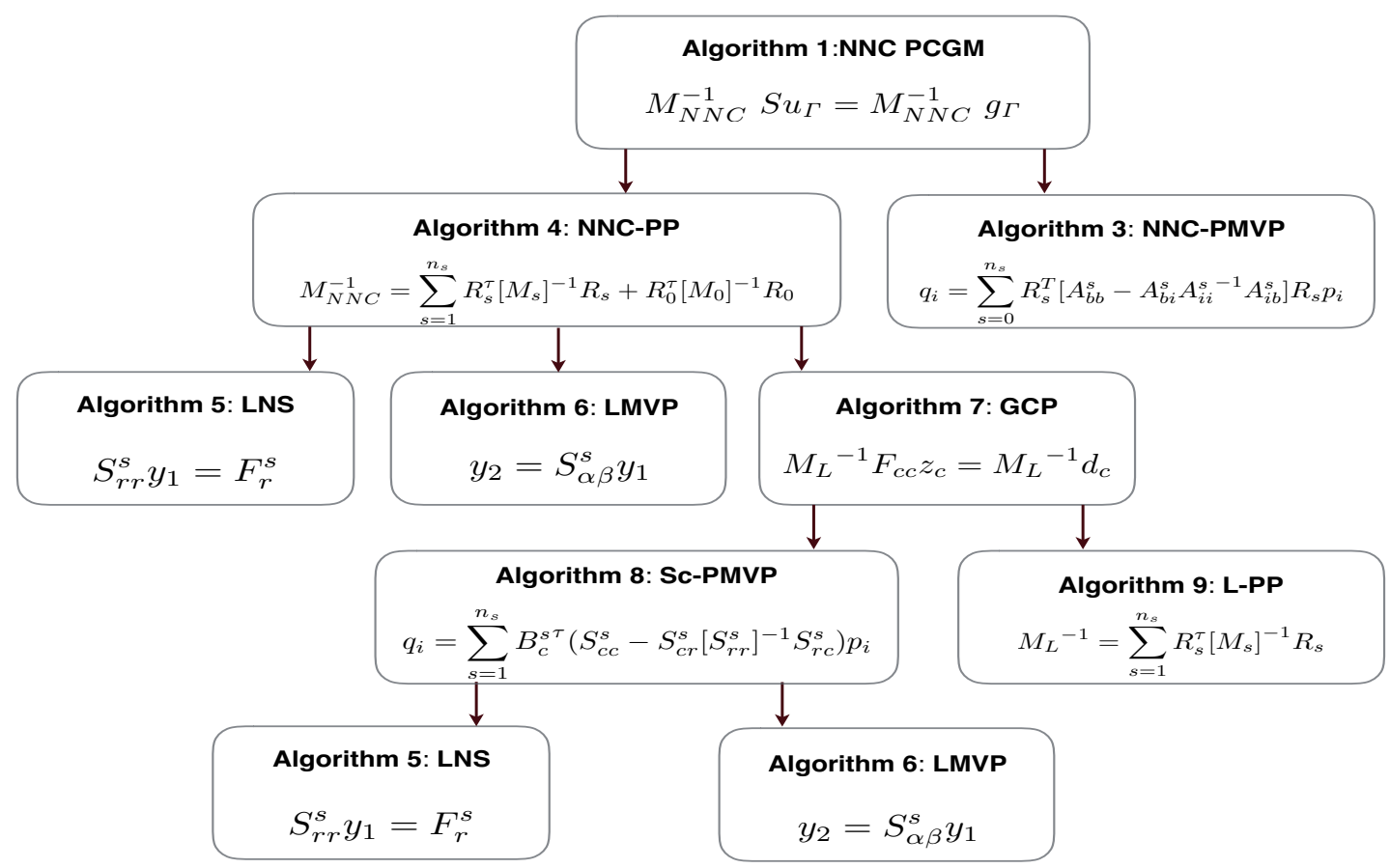

Figure 3.5: Flowchart for BDDC/NNC-PCGM solver.

operations. Hence, the parallel overhead is mainly due to collective MPI communications. Direct use of MPI helps in maintaining flexibility in parallel implementation of the solvers.

\subsubsection{Implementation of FETI-DP Algorithm using PETSc}

The preconditioned extended Lagrange multipliers system of the PCE coefficients in Eq. (3.53) is solved iteratively using parallel PCGM procedure given in Algorithm 1. The one-level Dirichlet preconditioner in Eq. (3.54) is employed to accelerate the convergence of the system. The key steps 7 and 13 in Algorithm 1 (highlighted in blue) are (a) the parallel matrix-vector product procedure and (b) the parallel Dirichlet preconditioning procedure. Efficient parallel implementation of these two procedures using sparse PETSc objects, routines and solvers is discussed in the next sections. 


\section{Parallel Matrix-Vector Product for FETI-DP}

In this solver, the parallel matrix-vector product (PMVP) procedure outlined in Algorithm 10 is different compared to the PMVP in the BDDC/NNC-PCGM solver detailed earlier in Algorithm 3. The global coarse problem in FETI-DP is solved during the PMVP procedure as compared to BDDC/NNC-PCGM, in which it was solved during the application of two-level parallel preconditioner. The PMVP procedure for FETI-DP is outlined in Algorithm 10 for the following equation:

$$
q_{i}=\left[F_{r r}+F_{r c}\left[F_{c c}\right]^{-1} F_{c r}\right] p_{i}
$$

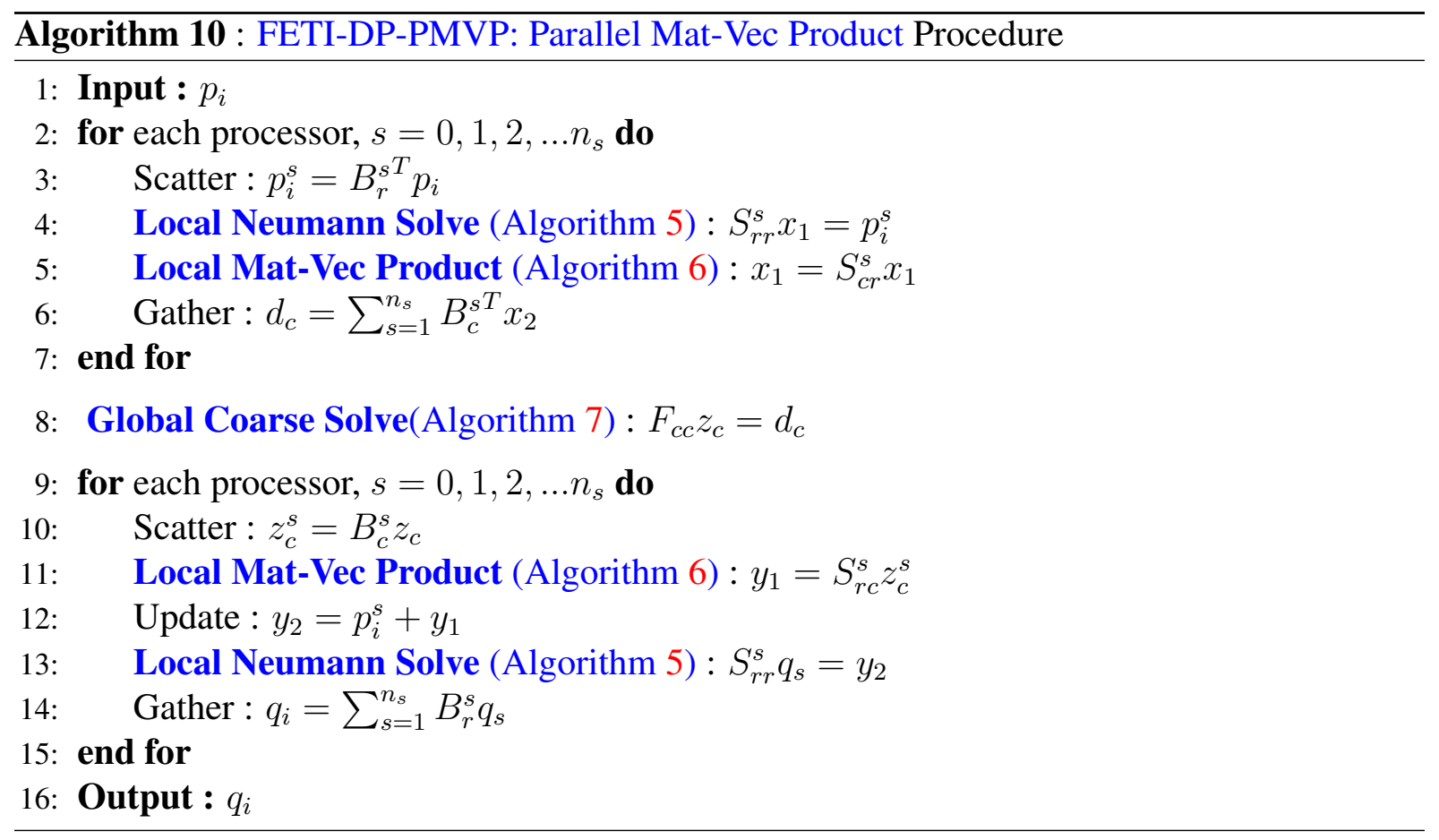

Similar to the BDDC/NNC-PCGM solver discussed previously, the key steps (highlighted in blue) in Algorithm 10 are implemented using PETSc. The local Neumann solve (LNS) procedure given by Algorithm 5 is used to solve the two local Neumann problems in steps 4 and 13 of Algorithm 10. The local Mat-Vec product (LMVP) procedure outlined in Algorithm 6 is employed to compute the two local matrix-vector products in step 5 an 11. For each main 
PCGM iteration, the global coarse problem (GCP) is solved iteratively using a coarse PCGM with the one-level lumped preconditioner outlined in Algorithm 7. The key procedures from the GCP (highlighted in blue) are implemented using Algorithm 8 and Algorithm 9 respectively for the Sc-PMVP and L-PP procedures.

\section{Parallel Preconditioning Procedure for FETI-DP-PCGM}

In the FETI-DP procedure, the system operator is already equipped with the coarse problem. Therefore, the one-level Dirichlet or lumped preconditioner is sufficient to obtain the scalable performance [18]. The parallel preconditioning effect using the Dirichlet preconditioner given in Eq. (3.54) is performed using the procedure outlined in Algorithm 11. In this procedure, the diagonal averaging matrix $D_{r}^{s}$ defined by Eq. (3.55) is applied twice: first on the local residual and then on the preconditioned residual. This procedure also requires one call to PETSc-KSPCG routine to solve the local interior problem.

$$
\begin{aligned}
z_{i} & =M^{-1} r_{i} \\
& =\sum_{s=1}^{n_{s}} B_{r}^{s} D_{r}^{s}\left[M_{s}\right]^{-1} D_{r}^{s} B_{r}^{s T} r_{i} .
\end{aligned}
$$

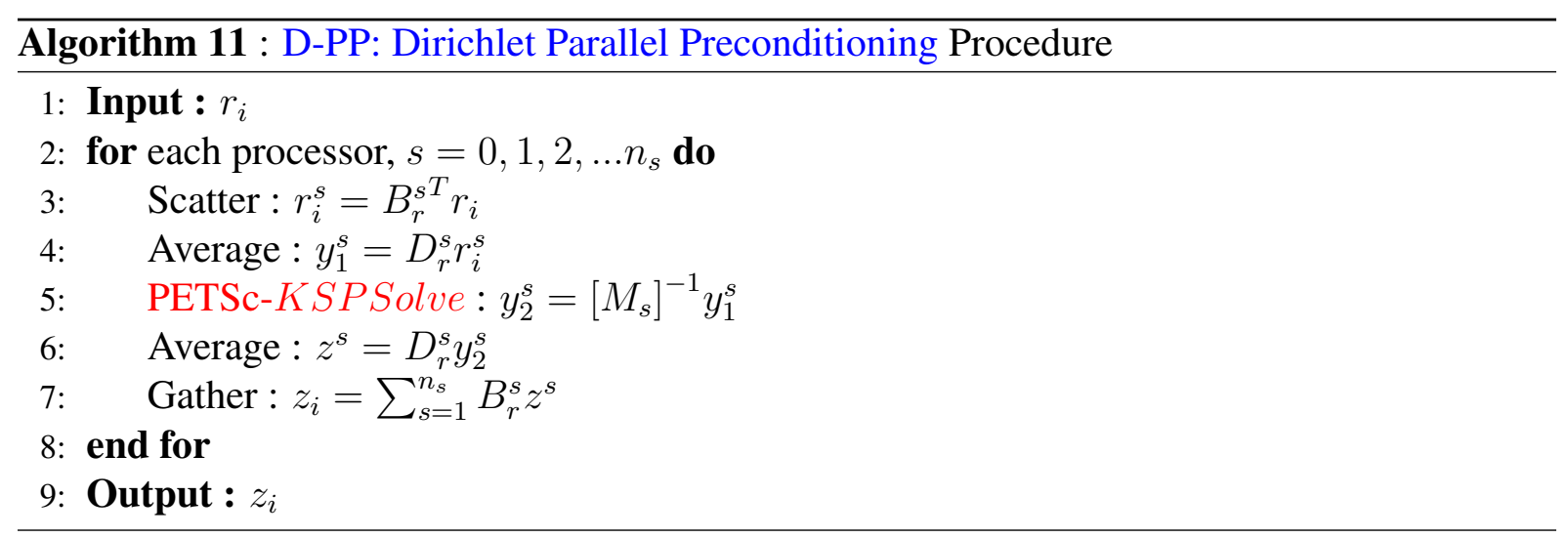

The flowchart detailing the implementation of the FETI-DP using PCGM is given in Fig. 3.6. As one can observe by comparing the flowcharts for BDDC/NNC-PCGM and FETI-DP-PCGM, 
the types of operations performed in the implementations of both solvers are similar. The main differences are: (a) the operations performed during the preconditioning procedure for BDDC/NNC-PCGM are now executed during the matrix-vector product procedure for FETI-DP and (b) the operations performed during the matrix-vector product procedure for NNC-PCGM are now carried out during the preconditioning procedure for FETI-DP-PCGM.

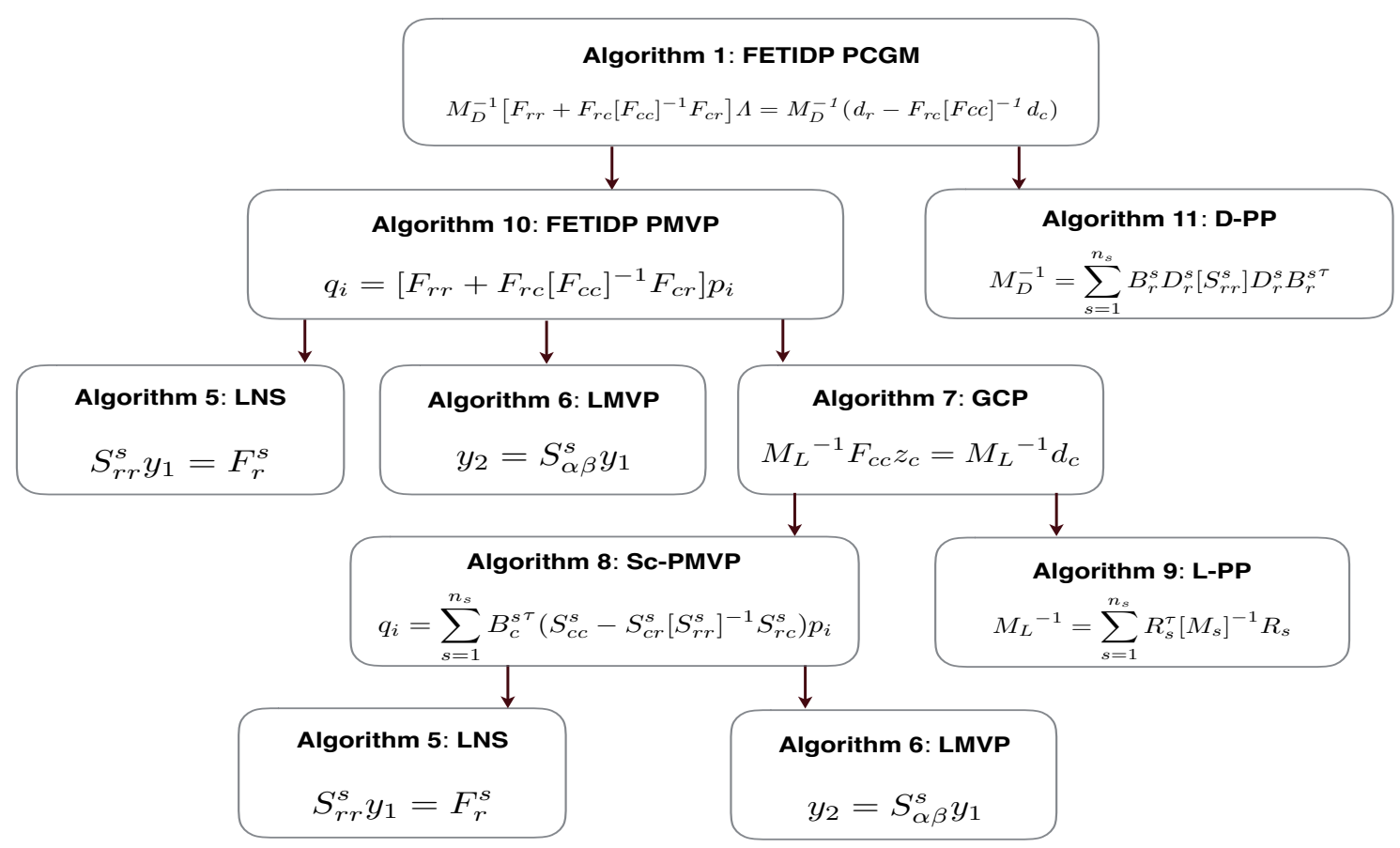

Figure 3.6: Flowchart for FETI-DP PCGM solver.

\subsection{Numerical Experiments}

In this section, we investigate the performance of BDDC/NNC-PCGM and FETI-DP-PCGM solvers based on the implementation techniques presented in Sec. 3.2.1. As a test case, we have considered two-dimensional steady-state flow through random media, modeled by a stochastic diffusion equation described earlier in Eq. (2.14). The spatially varying diffusion coefficient is modeled as a lognormal stochastic process, obtained from an underlying Gaussian field with an 
exponential covariance function given in Eq. (2.4). For this study, the numerical values of the parameters are provided next. The underlying Gaussian random field is assumed to have the zero mean, correlation lengths $b_{1}=b_{2}=1.0$ and the standard deviation $\sigma=0.3$. A constant unit source term, $\mathbf{f}=1$ is used. The physical domain with unit-square geometry is discretized using an unstructured FE mesh. A typical mesh with 121896 nodes and 243794 triangular elements partitioned into 1680 subdomains is shown in Fig. 3.7. The primary focus now is to investigate the performance of the solvers to handle the high-dimensional stochastic systems. In the subsequent sections, we will first discuss the experimental framework for the simulations followed by the results for numerical and parallel scalabilities of the proposed solvers.

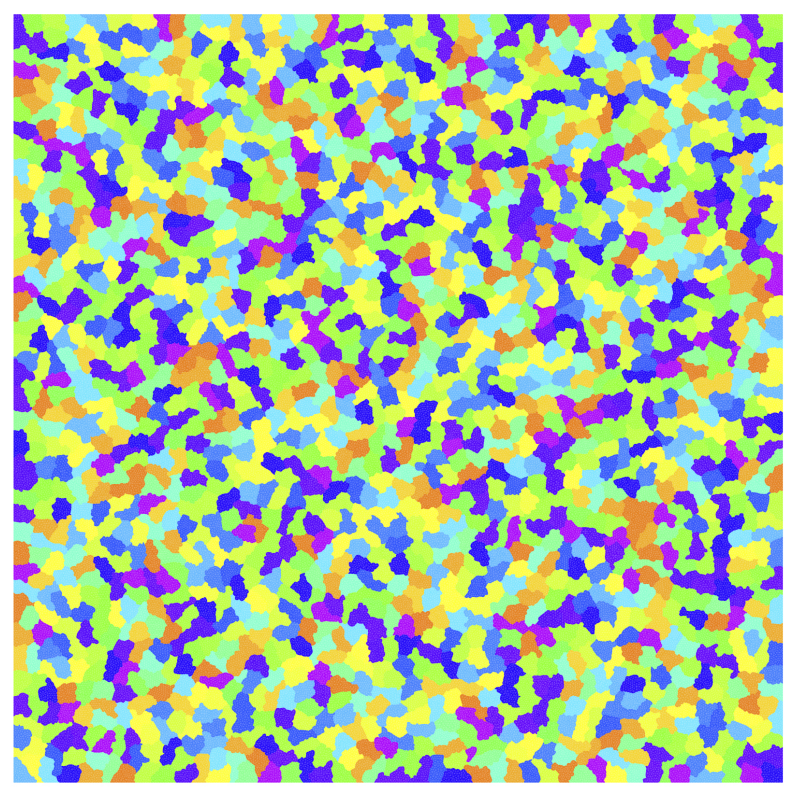

Figure 3.7: Typical finite element mesh with 1680 subdomains.

\subsubsection{Experimental Framework}

The algorithms presented in previous sections are implemented by the author using Fortran programming language. Parallel processing is managed by using Message Passing Interface (MPI) communication routines [33]. Data structures and routines from PETSc [31] are used for subdomain-level (local) sparse matrix-vector storage and algebraic operations such as local sparse matrix-vector multiplication. The finite element mesh is generated using GMSH [27,202] 
and partitioned using METIS graph partitioner [28,203]. Stochastic system matrix and vector assembly is performed by employing element-level (deterministic) assembly routines from the FEniCS/dolfin [32]. The procedures from UQ Toolkit [29] are utilized to evaluate KLE and PCE basis functions and the corresponding multidimensional moments. For post processing and visualization, ParaView [34, 204] and Matlab [35] are employed. Further details on the implementation of these steps with the code snippets are given in Appendix B.

The computations are performed on Canada's national HPC clusters managed by Compute Canada and Calcul Québec [205,206]. These HPC systems have all nodes and storage connected by low-latency, high-bandwidth Infiniband fabric interconnect [205] and we utilize MPI standards for communications [33]. The nodes employed have either Intel E5-2670 processors running at 2.6 GHz from Guillimin HPC system [206] or have Intel E5-2683 processors, running at 2.1 GHz from Cedar and Graham HPC systems [205]. For low-memory simulations (i.e., the cases with a few RVs), processors with 4 GB of RAM per core are used and for highmemory simulations (i.e., the cases with large number of RVs), processors with 8 GB of RAM per core are employed.

\subsubsection{Characteristics of the Solution Process}

As a test case, consider five random variables $(L=5)$ and the second order expansion $\left(p_{\mathrm{A}}=2\right)$ for the PCE of the input stochastic process. The PCE of the solution process with the five random variables and the third order expansion $\left(p_{u}=3\right)$ is used. For these parameters, we need 21 PCE terms for the input stochastic process and 56 PCE terms for the output stochastic process [1].

The mean, standard deviation and few selected higher-order PCE coefficients of the solution process are plotted in Fig. 3.8 and Fig. 3.9 respectively. The mean and standard deviation of the solution field $u$ has the same trend. The maximum coefficient of variation $(\mathrm{CoV})$ of the solution process is about $24 \%$. The magnitude of the chaos coefficients $u_{j}$ decreases with increasing PCE index $j$, i.e., the contribution of the higher-order PCE coefficient to the solution process 


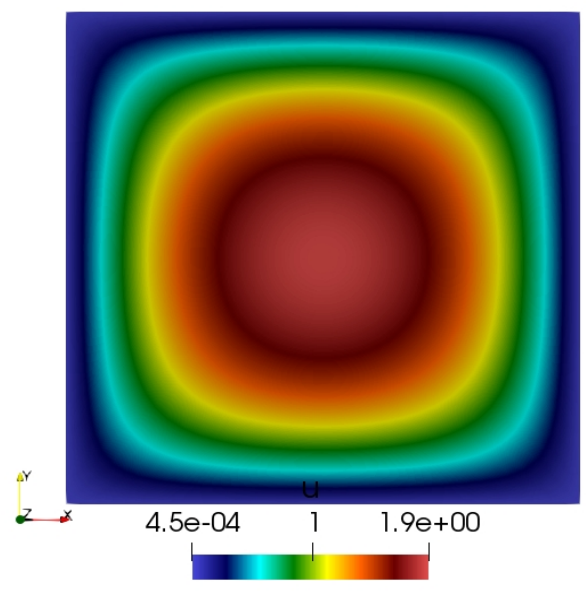

(a) mean $u$

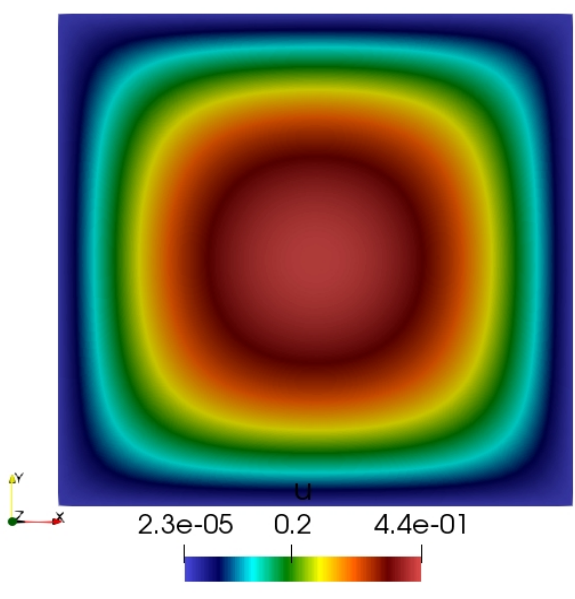

(b) standard deviation $u$

Figure 3.8: Mean and standard deviation of the solution process $u$

decreases. As the order of expansion increases, the solution converges in terms of mean and standard deviation. Among these PCE coefficients, the first order coefficients contain Gaussian contributions and the higher-order coefficients contain the non-Gaussian effects.

\subsubsection{Scalabilities Studies for Stochastic Simulations}

The main purpose of this study is to investigate the performance of the proposed solvers for the problems with high dimensions in stochastic space. Therefore, for most of the simulations, the finite element mesh with the fixed number of nodes 52704 and elements 105410 is used $[17,18]$. For some cases, the mesh size is increased up to 0.332 million nodes and 0.664 million elements.

To investigate the high-dimensional stochastic spaces, we need a large number of random variables to represent the underlying random process. Hence, various experiments are performed with increasing number of random variables, i.e. number of KLE modes used to represent the underlying Gaussian process of the lognormal diffusion coefficient. We present the performance plots for various cases by selecting the number of random variables ranging between 3 to 20 . These cases are selected based on the relative contribution of the KLE eigenvalues. The relative partial sums of the eigenvalues of the two-dimensional exponential covariance function 


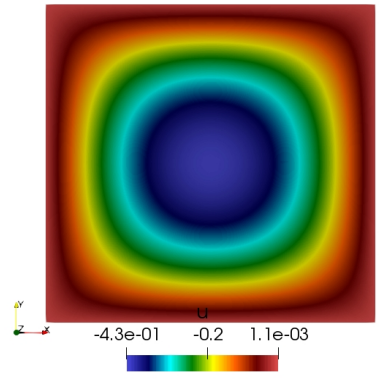

(a) $u_{1}$

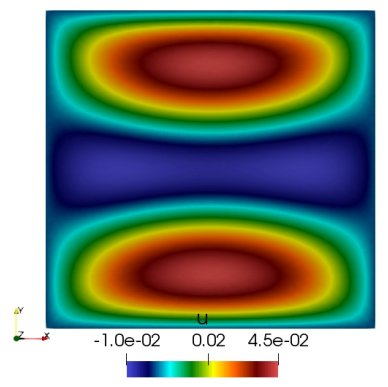

(d) $u_{5}$

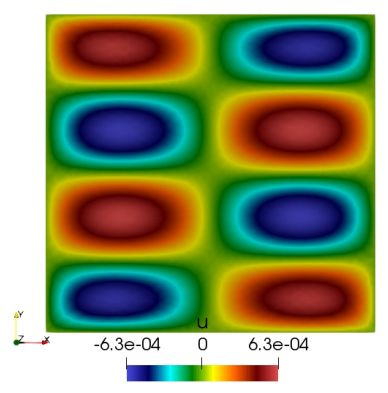

(g) $u_{19}$

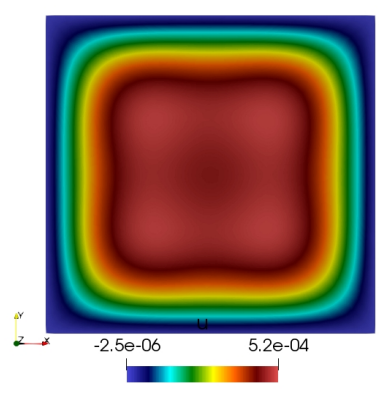

(j) $u_{41}$

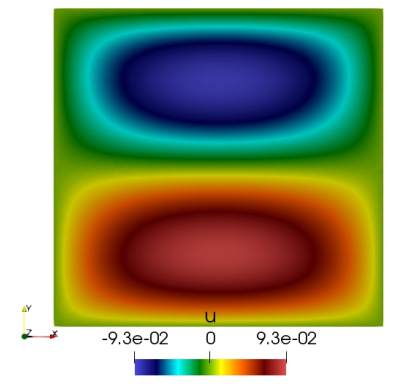

(b) $u_{2}$

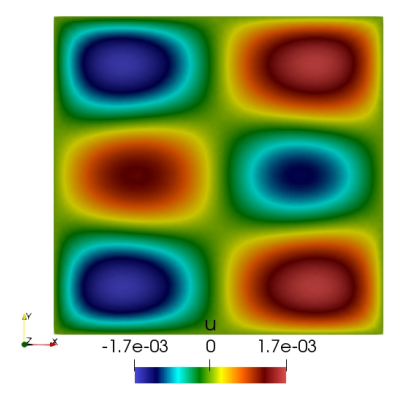

(e) $u_{13}$

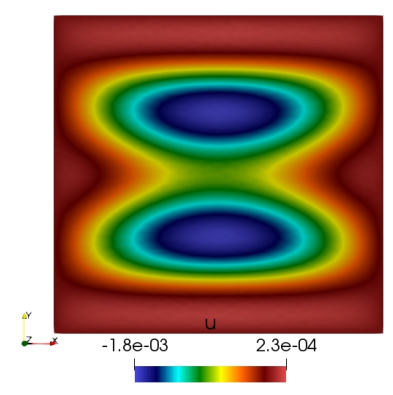

(h) $u_{20}$

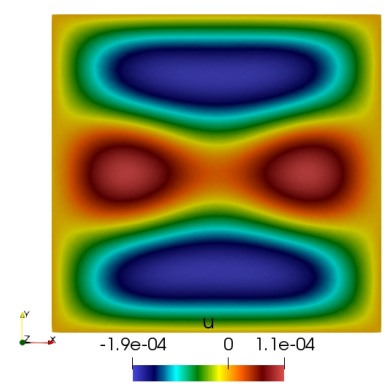

(k) $u_{48}$

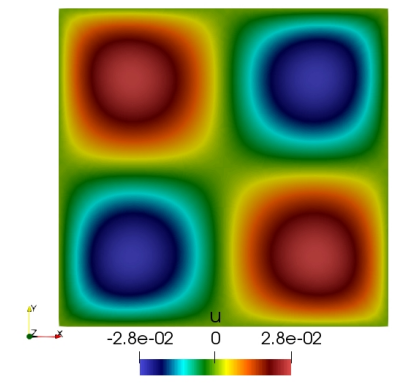

(c) $u_{4}$

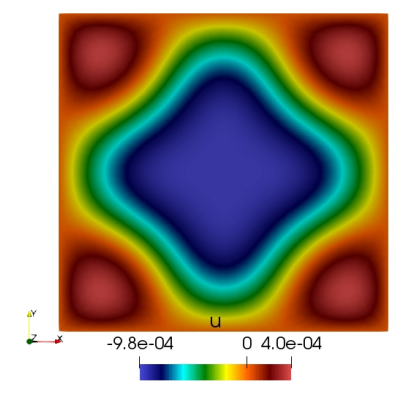

(f) $u_{18}$

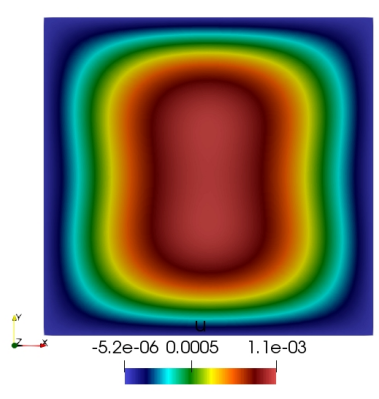

(i) $u_{35}$

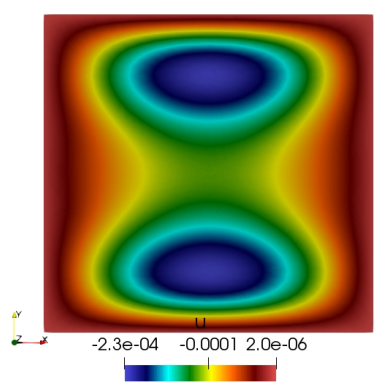

(l) $u_{55}$

Figure 3.9: Selected PCE coefficients of the solution process $u$ 


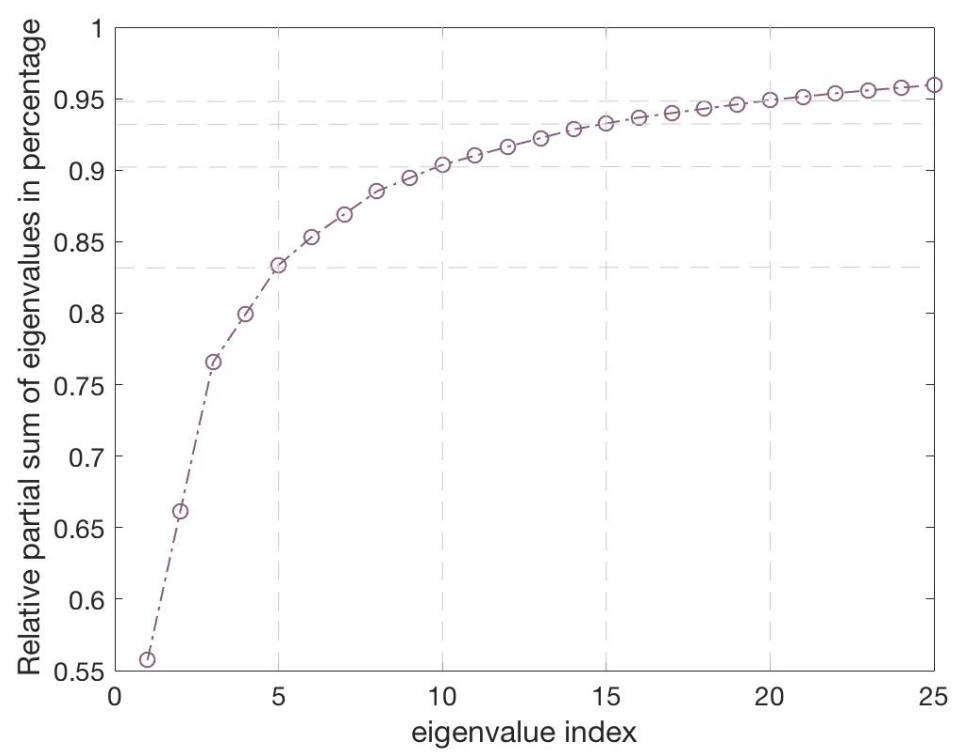

Figure 3.10: Relative partial sum of eigenvalues with respect to eigenvalue-index.

with $b_{1}=b_{2}=1.0$ is plotted in Fig. 3.10. First ten eigenvalues account for about $90 \%$ of the energy of the random field and first twenty eigenvalues correspond to about $95 \%$ of the energy of the random field (refer [1, 17, 18] for further details).

The orders of expansion $p_{\mathrm{A}}=2$ and $p_{u}=3$ are used for the input and output PCE representations respectively. Note that the orders of expansion for the input and output PCE is kept constant for most of the experiments. This is mainly to keep the focus of the investigation on the problems with high-dimensional stochastic spaces. For some cases $p_{u}$ is varied between 2 to 4 . With these KLE and PCE inputs, the number of PCE terms required to represent the solution process varies from 20 to 1771 [1]. Using approximately 122 thousand spatial node points with 1771 PCE terms corresponding to 20-KLE modes leads to the linear system of 216 million approximately. In such cases, the memory required to accommodate the problem in a given computer becomes a critical issue.

The memory usage per core in Fig. 3.11 is computed by averaging the actual memory used by each core. It compares the computational resources used for the investigation. For these experiments, the mesh with 102010 nodes and 202910 elements is used. The number of PCE 


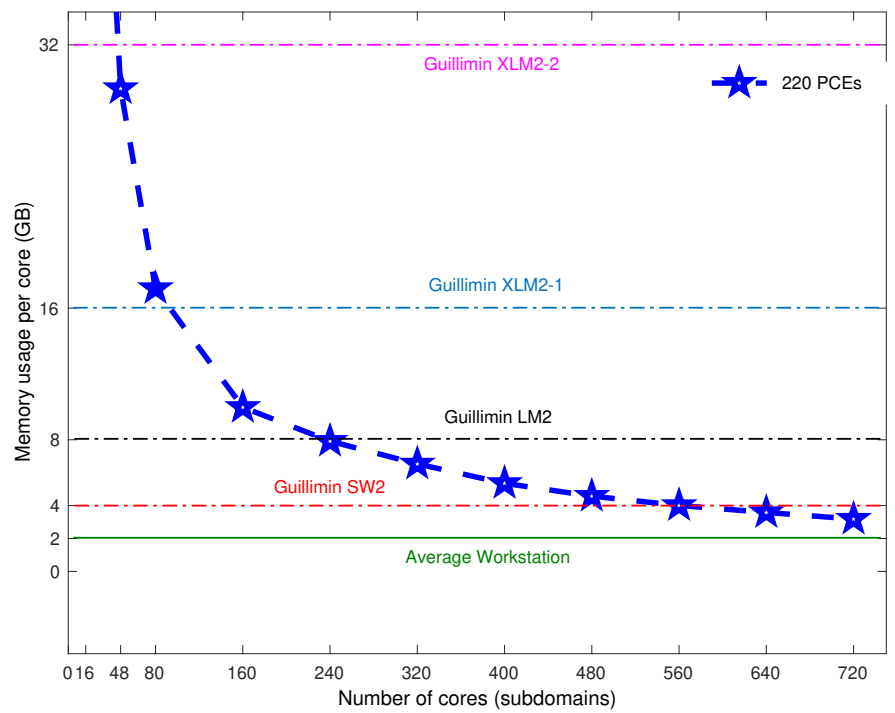

Figure 3.11: Need of DDM based solvers with the aid of computer cluster for stochastic simulations.

terms $P_{u}$ for the solution process is 220 . As evident from the plot, even with 720 partitions one can not solve this problem in a HPC cluster having 2 to 3 GB RAM per core. For fewer subdomains, for instance, for 48 subdomains, one needs a cluster with large memory per core such as Guillimin extra large memory nodes (XLM2) [206] having nodes with 32 GB of RAM per core. We require at least 240 subdomains to solve this problem using a cluster having roughly 8 GB RAM per core (such as Guillimin large memory nodes (LM2) [206]) and more than 500 subdomains for a cluster having roughly 4 GB RAM per core (Guillimin SW2 [206]). This leads to the important outcome that the DDM with high performance computing is a promising way to tackle the high-dimensional stochastic systems. Hence this fact opens up the possibility to overcome the curse of dimensionality in stochastic systems.

\subsubsection{Scalability Results for BDDC/NNC-PCGM Solver}

This section presents the scalability results for BDDC/NNC-PCGM solver discussed previously. First, we study the numerical scalability concerning the number of PCGM iterations required for the convergence with the tolerance: $\frac{\left\|u_{\Gamma_{i+1}}-u_{\Gamma_{i}}\right\|_{2}}{\left\|u_{\Gamma_{i}}\right\|_{2}} \leq t o l=10^{-5}$ with the subscript indicating 
the PCGM iteration number. Next, we examine the (strong and weak) parallel scalabilities in terms of time against the number of subdomains. Finally, we present the numerical and parallel scalability plots with respect to the stochastic parameters such as the number of random variables, order of expansion, variance and correlation lengths.

\section{Numerical Scalability}

For the numerical scalability study, we examine the number of PCGM iterations against the number of subdomains. For a fixed problem size with fixed number of PCE terms $\left(P_{u}=816\right)$, if we increase the number of subdomains, the PCGM iteration count remains constant. Fig. 3.12 indicates that the BDDC/NNC-PCGM solver is numerically scalable with respect to the number of subdomains.

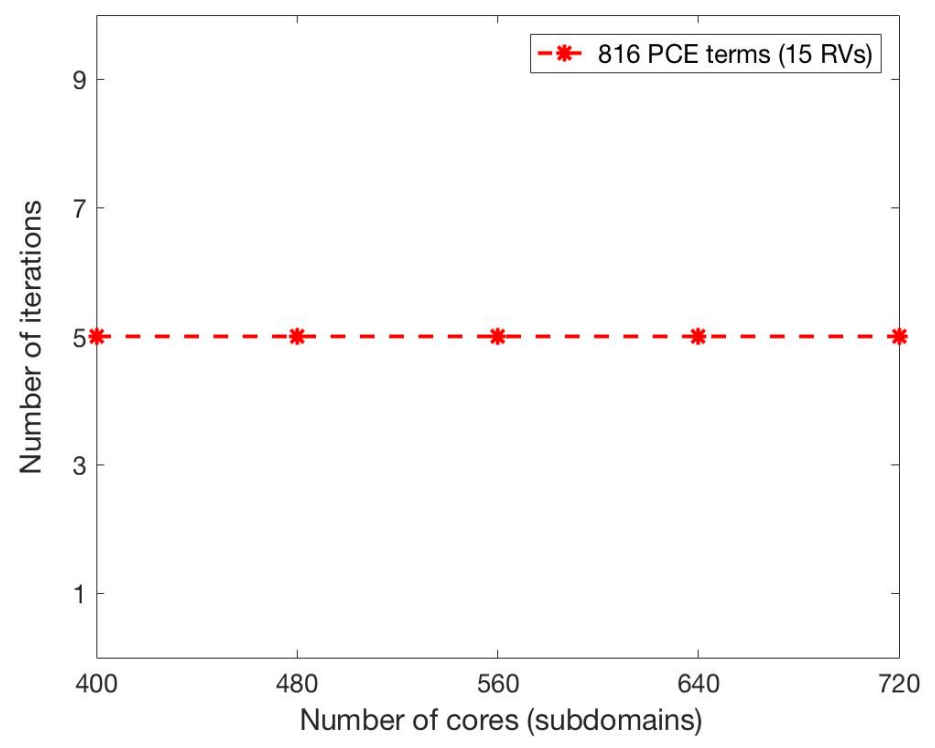

Figure 3.12: Iteration count versus number of subdomains for the fixed mesh resolution with fixed number of PCE terms.

Next, for the fixed number of PCE terms $\left(P_{u}=816\right)$, the global problem size is increased by increasing mesh resolution while keeping a fixed problem size per subdomain $(\approx 60 K)$, the PCGM iteration count remains constant. Fig. 3.13 indicates that the BDDC/NNC-PCGM solver is numerically scalable with respect to mesh resolution and fixed problem size per subdomains. 


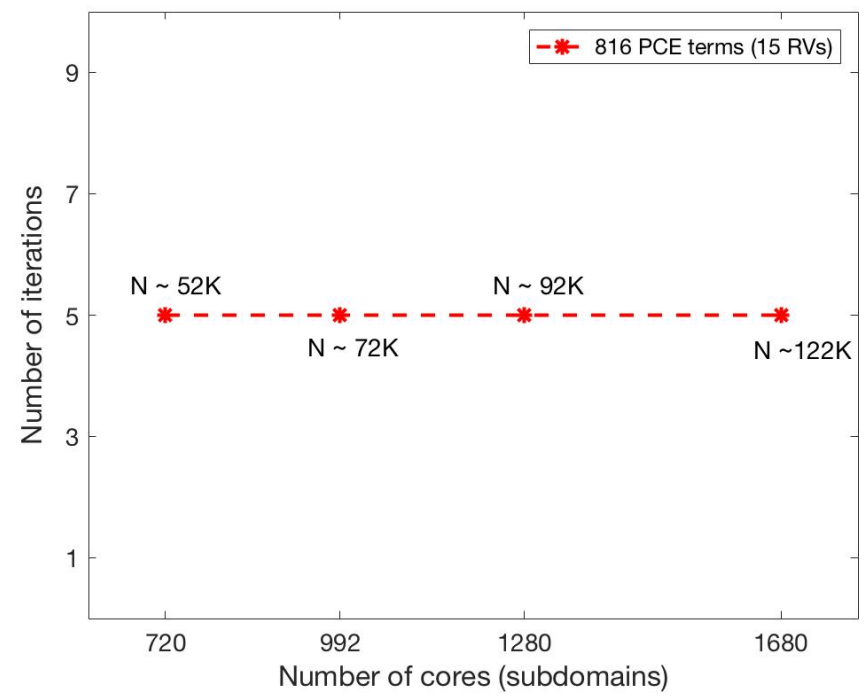

Figure 3.13: Iteration count versus number of subdomains for the fixed problem size per core with increasing mesh resolution (fixed number of PCE terms).

\section{Parallel Scalabilities}

The strong scalability of the BDDC/NNC-PCGM solver is plotted in Fig. 3.14. For fixed spatial mesh resolution with various number of PCE terms (ranging from 286 to 1771), an increase in the number of cores reduces the execution time as expected. Fig. 3.14 shows three cases corresponding to 286,816 and 1771 PCE terms arising from 10, 15 and 20 random variables respectively. The mesh resolution being fixed, Fig. 3.14 indicates the strong scalability of the solver with respect to the number of random variables. On the other hand, for a fixed number of cores, the problem size per core grows as the number of PCE terms increases. In this case, the communication topology remains the same but the amount of (computational) data to be communicated per core for each PCGM iteration grows.

The weak scalability of the BDDC/NNC-PCGM solver is plotted in Fig. 3.15. For the fixed number of PCE terms $\left(P_{u}=816\right)$, the global problem size is increased by increasing mesh resolution while keeping a fixed problem size per subdomain. The growth in the total execution time implies that the probabilistic BDDC/NNC shows poor weak scalability due to a simple parallel implementation using MPI collective communication (i.e., communication among all processes). This behavior can be improved by using MPI point-to-point communication [33]. 


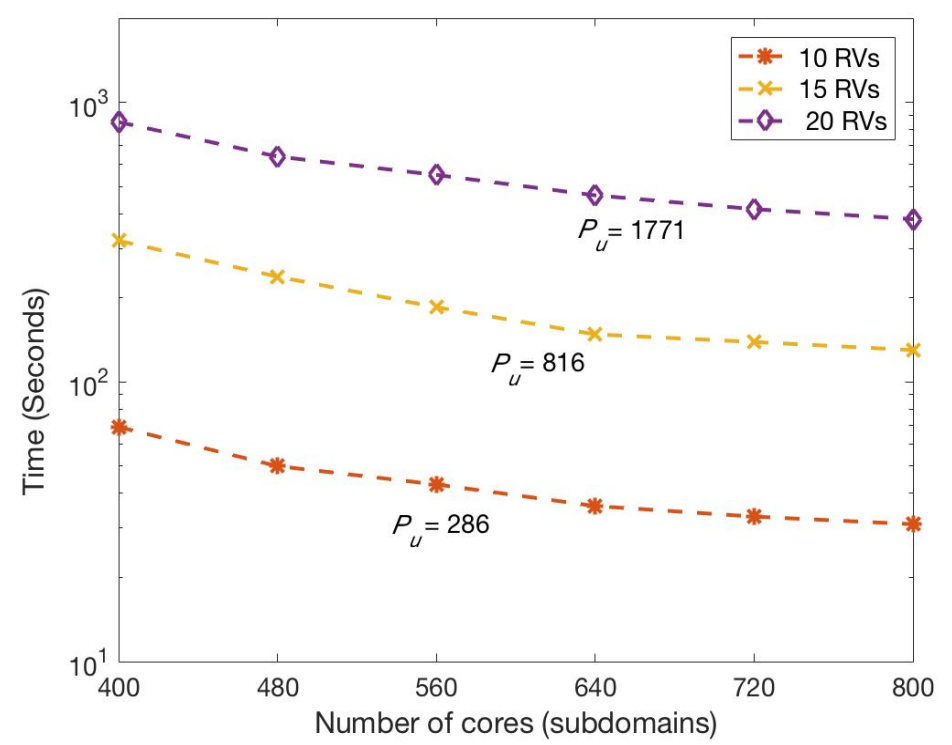

Figure 3.14: Execution time versus number of subdomains with the fixed mesh resolution versus the number of PCE terms.

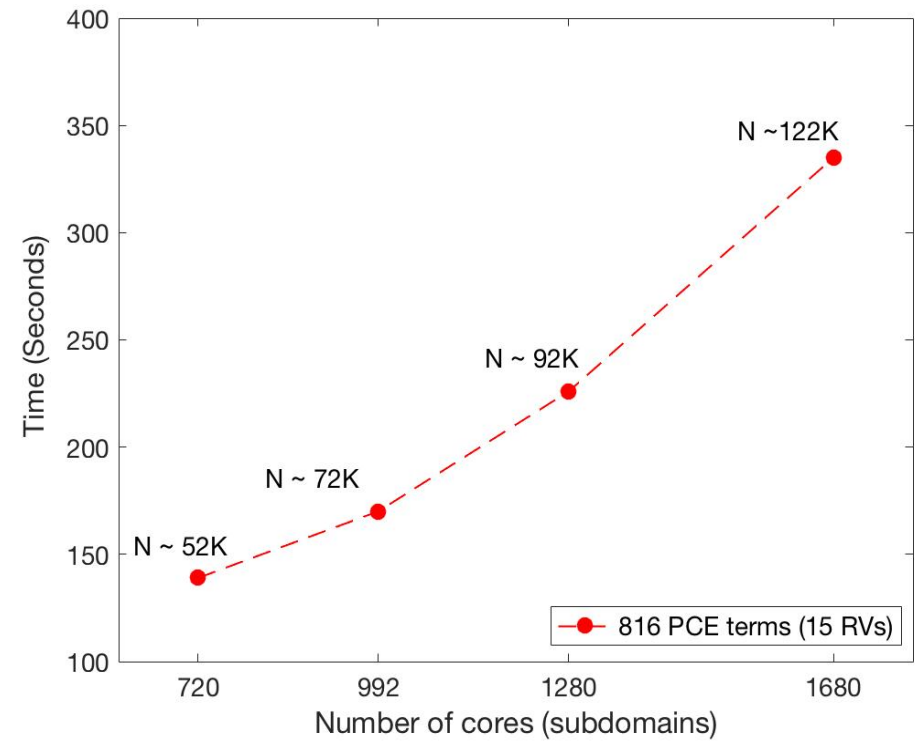

Figure 3.15: Execution time versus number of subdomains for the fixed problem size per core with increasing mesh resolution (fixed number of PCE terms). 


\section{Scalability with respect to Stochastic Parameters}

In this section, numerical and parallel scalabilities with respect to the number PCE terms are presented. These studies are performed in order to investigate the effects of stochastic aspects on the scalabilities of the solvers. The four points in Figs. 3.16 to 3.18 corresponding to the number of PCE terms 56, 286, 816 and 1771 are related to the number of random variables 5, 10, 15 and 20 respectively. The finite element mesh resolution corresponds to 52704 nodes and 105410 elements.

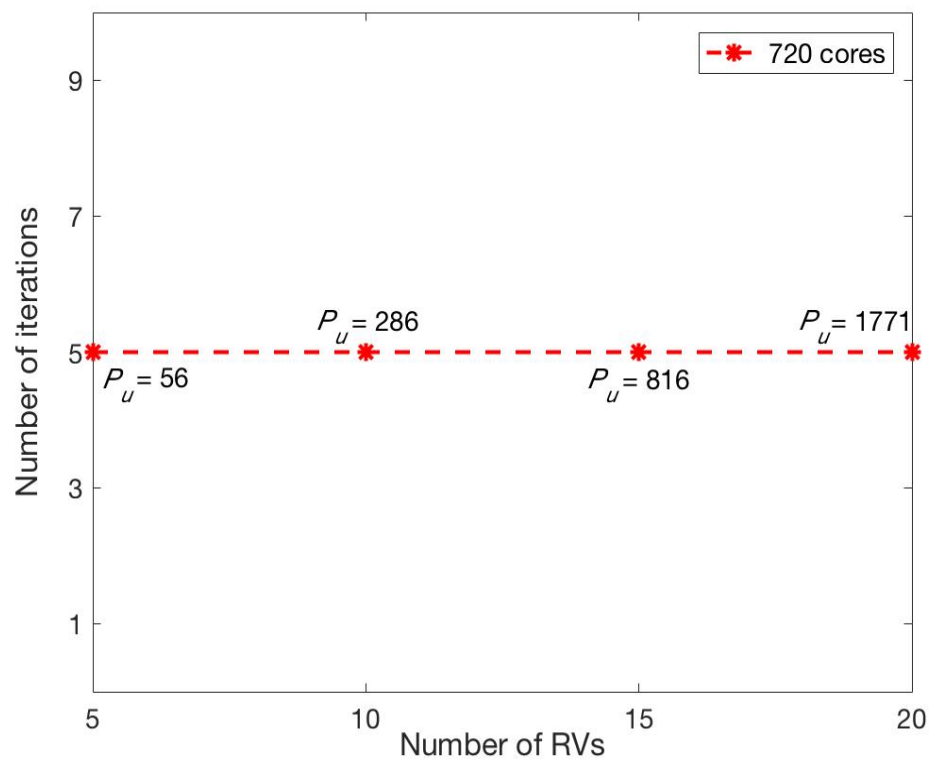

Figure 3.16: Iteration count versus number PCE terms for the fixed mesh resolution with fixed number of subdomains.

For a fixed spatial resolution and fixed number of subdomains $\left(n_{s}=720\right)$, an increase in the number of PCE terms (while maintaining the third-order expansion) by increasing the number of random variables results in constant PCGM iteration counts required for convergence, as shown in Fig. 3.16. This result shows that the BDDC/NNC-PCGM solver is numerically scalable with respect to the number of random variables. Although the total problem size increases with the number of PCE terms, one can solve the larger system with the same number of PCGM iterations. 


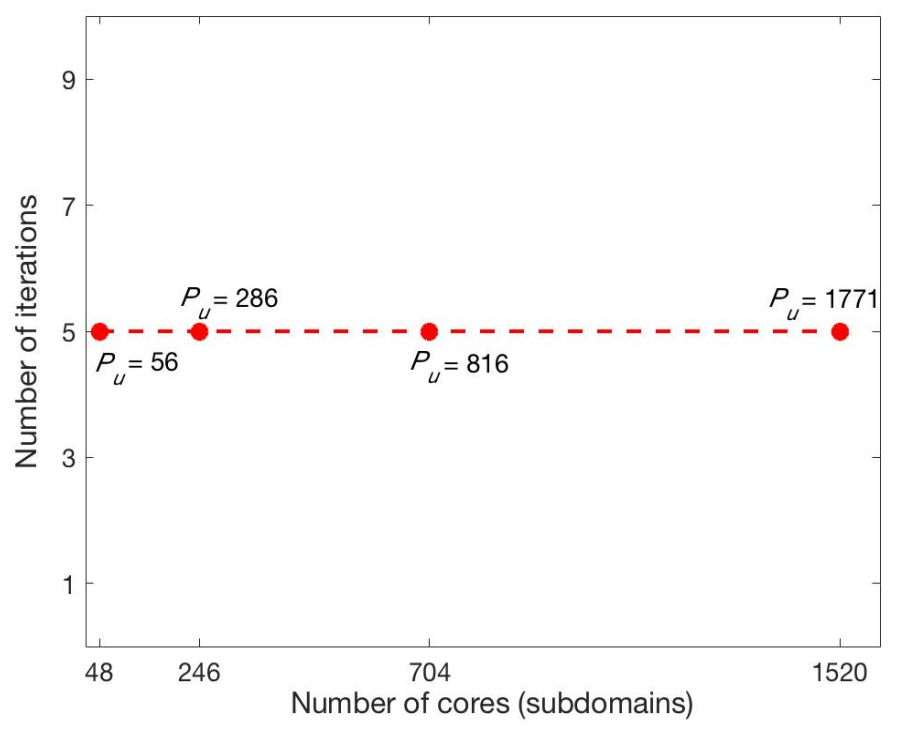

Figure 3.17: Iteration count versus number of subdomains for the fixed problem size per core with increasing number of PCE terms (fixed mesh resolution).

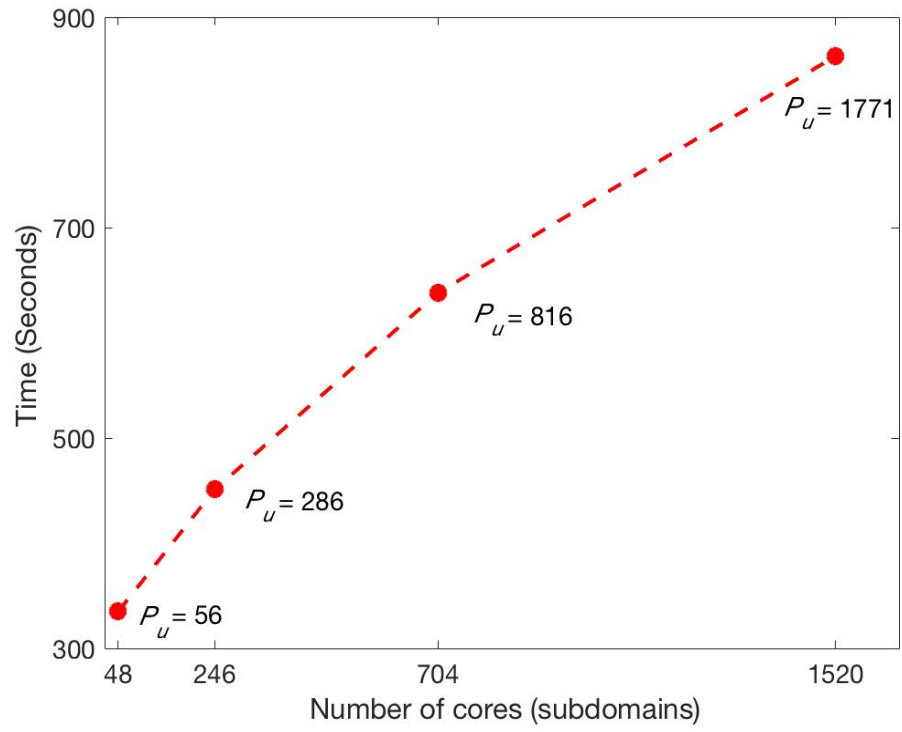

Figure 3.18: Execution time versus number of subdomains for fixed problem size per subdomain with increasing number of PCEs (fixed mesh resolution). 
With the increasing number of PCEs, the global problem size increases for a fixed mesh resolution. The ensuing larger linear system is then solved using more cores by keeping the problem size per core approximately the same $(\approx 60000)$. The iteration count for the BDDC/NNCPCGM solver versus the number of subdomains (with the fixed problem size per subdomain) remains constant as shown in Fig. 3.17. Hence the BDDC/NNC-PCGM solver is numerically scalable with respect to the number of PCEs. The total execution time for the solver against the number of subdomains, with fixed problem size per subdomain, is illustrated in Fig. 3.18. The execution time increases with respect to the PCE terms and the number of subdomains although the problem size per core is nearly the same. This suboptimal parallel scalability with respect to the number of PCE terms can be due to the fact that, in the current implementation, communication among the subdomains is performed globally using MPI collective routines (scatter and gather) [33]. This can be partially alleviated in the future implementation by using MPI point-topoint communication routines (send and receive) whenever applicable [33]. To further improve the weak scalabilities, the communicator-aware and interlevel-overlapped message-passing implementation can be employed using MPI subcommunicators suggested in [207]. These changes show promising results for the deterministic BDDC solvers at extreme-scale [201, 207]. Such changes will be incorporated in the future implementation of these stochastic DDM solvers.

Additionally, the increasing number of PCE terms leads to a larger system of coupled equations and the new system inherits a more complex coupling structure (for instance, see SSFEM system matrices given in Fig. 2.3 and 2.4). The new coupling structures resulting from increasing number of PCE terms by increasing the number of random variables are more complex and computationally demanding than those resulting from increasing order of expansion. The initial investigation suggests that the new coupling structure influences the condition number of the system; and therefore the new system demands more computational effort.

For a fixed mesh resolution and fixed number of subdomains $\left(n_{s}=400\right)$, an increase in the number of PCE terms by increasing the order of expansion $p_{u}$ (while maintaining the fixed number of random variables $L=5$ ) results in constant PCGM iteration counts as shown in 


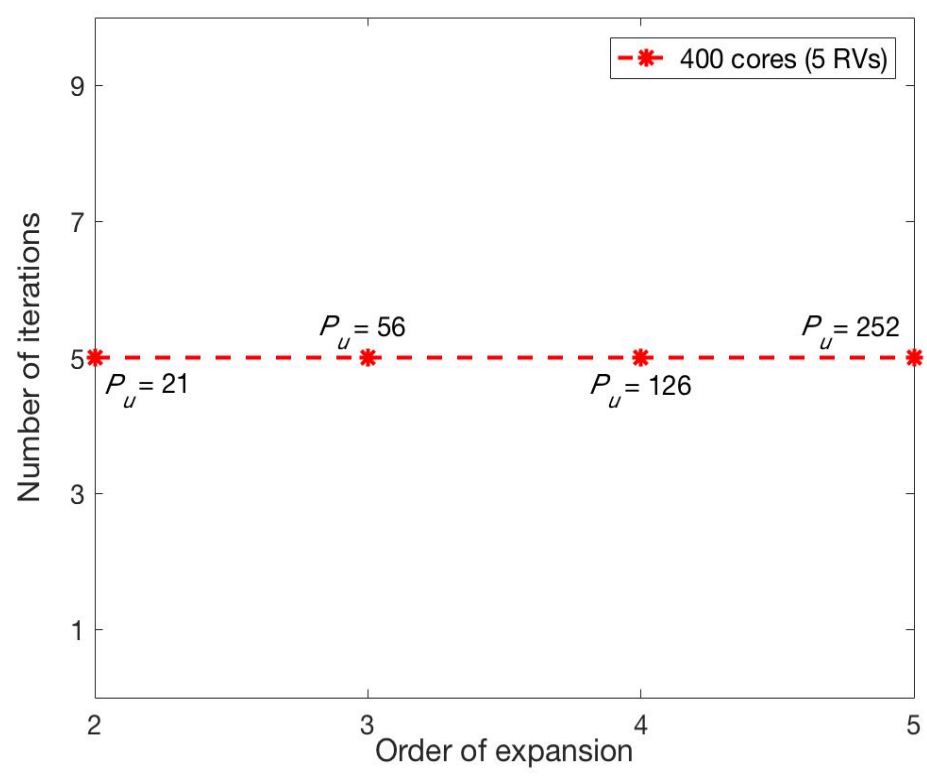

Figure 3.19: Iteration count versus order of expansion for the fixed mesh resolution with fixed number of subdomains (fixed number of RVs).

Fig. 3.19. This result shows that the BDDC/NNC-PCGM solver is numerically scalable with respect to the order of expansion. Note that, although the results are not shown for the sake of brevity, the change in the correlation lengths, $b 1=b 2=1.0$ to $b 1=b 2=0.3$ and standard deviation, $\sigma=0.3$ to $\sigma=0.6$ of the underlying stochastic process does not influence the performance of the solver.

\section{Scalability using Large-Scale HPC Cluster}

In a second set of experiments, we study the numerical scalability of the BDDC/NNC-PCGM solver for a highly dense mesh having 0.332 million nodes and 0.664 million elements. The number of cores utilized for these experiments vary from 1000 to 4000 . The number of PCE terms ranges from 20 to 220 and the order of expansion $p_{u}=3$ is kept constant. The scalability results with respect to the number of subdomains and the number of PCE terms are plotted in Fig. 3.20 and Fig. 3.21 respectively. These plots indicates that the solver is numerically scalable with large-scale computing clusters. Similarly, the results in Fig. 3.20 shows an excellent strong parallel scaling with thousands of cores. 


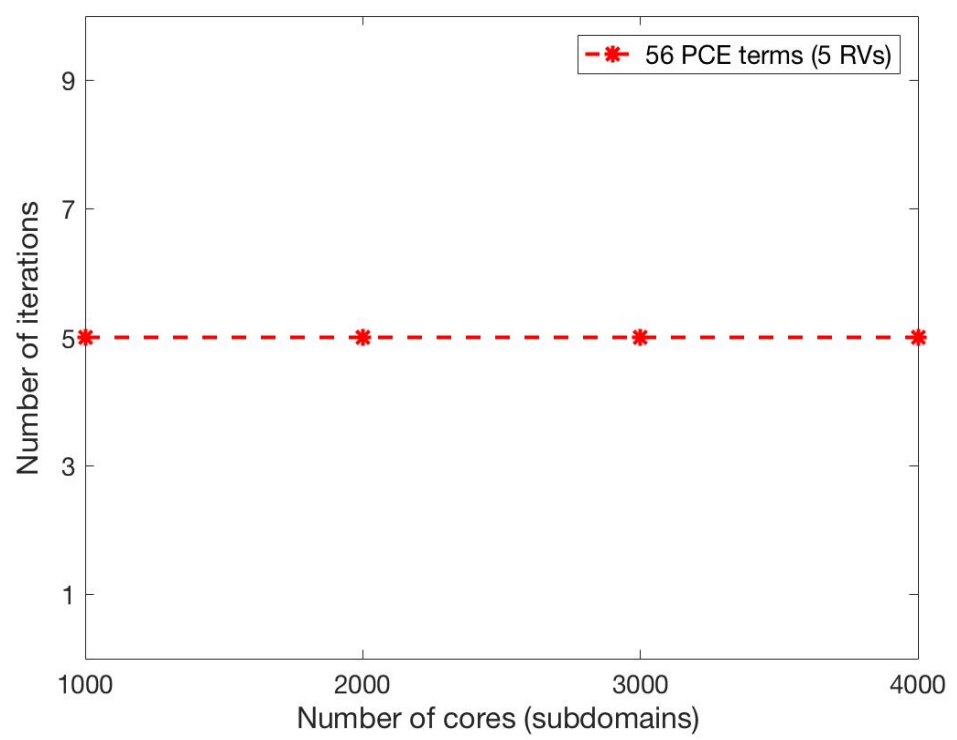

Figure 3.20: Iteration count versus number of subdomains for the fixed mesh resolution with fixed number of PCE terms.

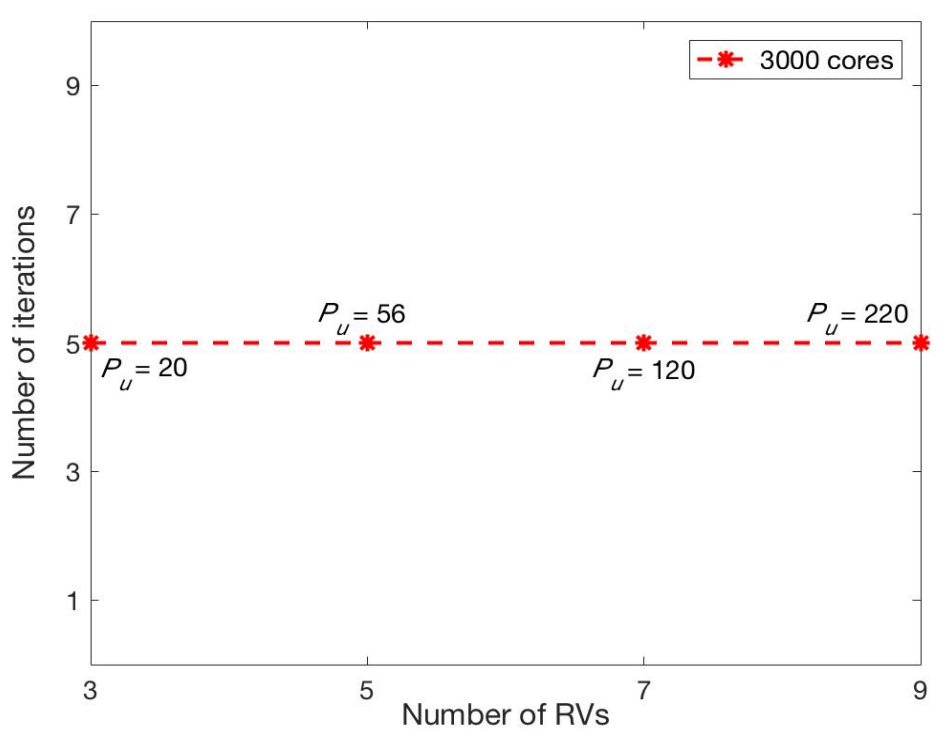

Figure 3.21: Iteration count versus number PCE terms for the fixed mesh resolution with fixed number of subdomains. 


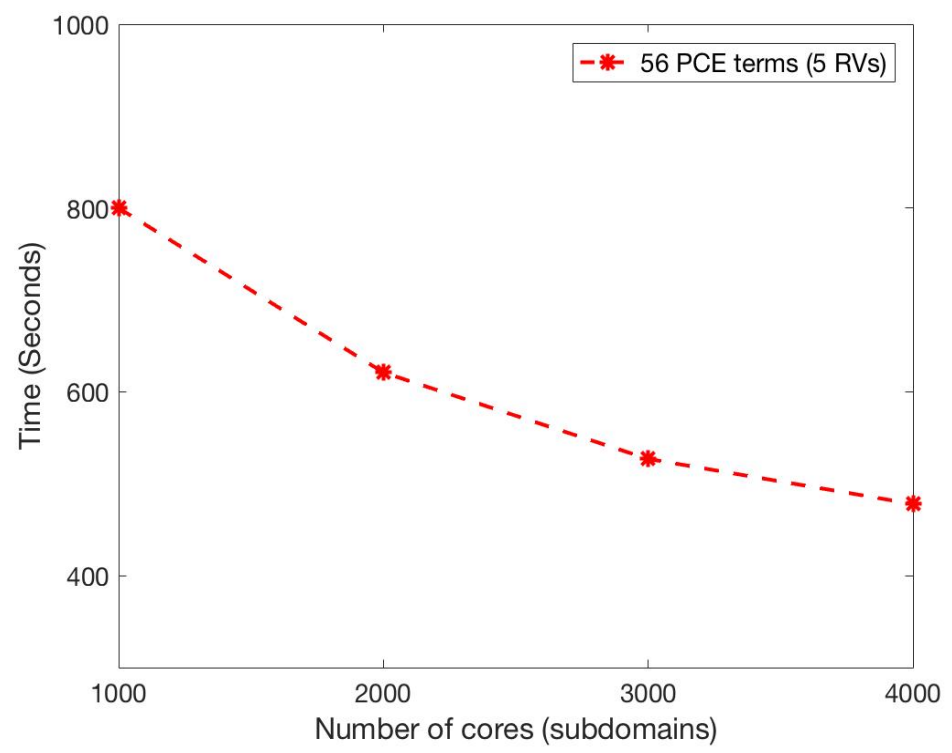

Figure 3.22: Execution time versus number of subdomains with the fixed mesh resolution and the fixed number of PCEs.

Furthermore, using PETSc-log-view, the total floating-point operations of all cores, average and maximum/minimum floating-point operations among cores are estimated against various numbers of cores used to solve the SSFEM system in the second set of experiments. The results are summarized in Table 3.1 and 3.2 below. Table 3.1 indicates, for a fixed problem size (both mesh resolution and the number of PCE terms are fixed), if we increase the number of cores, the total and average floating-point operations count decreases due to the decrease in workload per core as more cores are engaged. Table 3.2 indicates, for a fixed number of cores $\left(n_{s}=4000\right)$, both average and total floating-point operations count increases with the increasing number of PCE terms, emphasizing the increased workload per core. The total floating-point operations required in the case of $P_{u}=364$ reached 1.728 Petaflop. The results emphasize the necessity of using large-scale computing clusters and importance of scalable solvers for uncertainty propagation. Further increase in the number of RVs could demand a computing cluster capable of $10^{18}$ floating-point operations, pointing to emerging exascale computing [165,166]. From the perspective of load balancing, the maximum to minimum floating-point operations ratio 
among the cores is far from 1 indicating workload imbalance. Further investigation is needed to fine-tune the parallel implementation to achieve efficient load balancing.

\begin{tabular}{lccc}
\hline Fixed PCE $\left(P_{u}=56\right)$ & Total (Petaflop) & Average (Teraflop) & Max/Min \\
\hline$N_{s}=1000$ & 0.1684 & 0.1684 & 1.87000 \\
\hline$N_{s}=2000$ & 0.1255 & 0.0627 & 1.99444 \\
\hline$N_{s}=3000$ & 0.1195 & 0.0395 & 1.78914 \\
\hline$N_{s}=4000$ & 0.1201 & 0.0300 & 1.85542 \\
\hline
\end{tabular}

Table 3.1: Floating-point operations for different number of cores with the fixed number of PCE terms

\begin{tabular}{lccc}
\hline Fixed cores $\left(N_{s}=4000\right)$ & Total (Petaflop) & Average (Teraflop) & Max/Min \\
\hline$P_{u}=56$ & 0.1232 & 0.0308 & 1.85542 \\
\hline$P_{u}=120$ & 0.7884 & 0.1971 & 2.00349 \\
\hline$P_{u}=220$ & 1.0448 & 0.2612 & 1.99514 \\
\hline$P_{u}=364$ & 1.7281 & 0.4320 & 1.85249 \\
\hline
\end{tabular}

Table 3.2: Floating-point operations for different number of PCE terms with the fixed number of cores

\subsubsection{Scalability Results for FETI-DP-PCGM Solver}

The probabilistic version of FETI-DP-PCGM solver shows similar scalability results to those of BDDC/NNC-PCGM solver. For the sake of completeness, we have included some of the numerical scalability plots.

The number of PCGM iterations against number of PCE terms and number of subdomains are shown in Fig. 3.23 and Fig. 3.24 respectively. The iteration counts remain the same, demonstrating the numerical scalability of the probabilistic FETI-DP PCGM solver. The number of PCGM iterations against number of subdomains with the fixed problem size per subdomain is shown in Fig. 3.25. The similarity in performance is attributed to the resemblance between the type and number of operations performed during implementation of BDDC/NNC-PCGM and FETI-DP-PCGM solvers. These similarities become evident by comparing the flowcharts given in Fig. 3.5 and Fig. 3.6. 


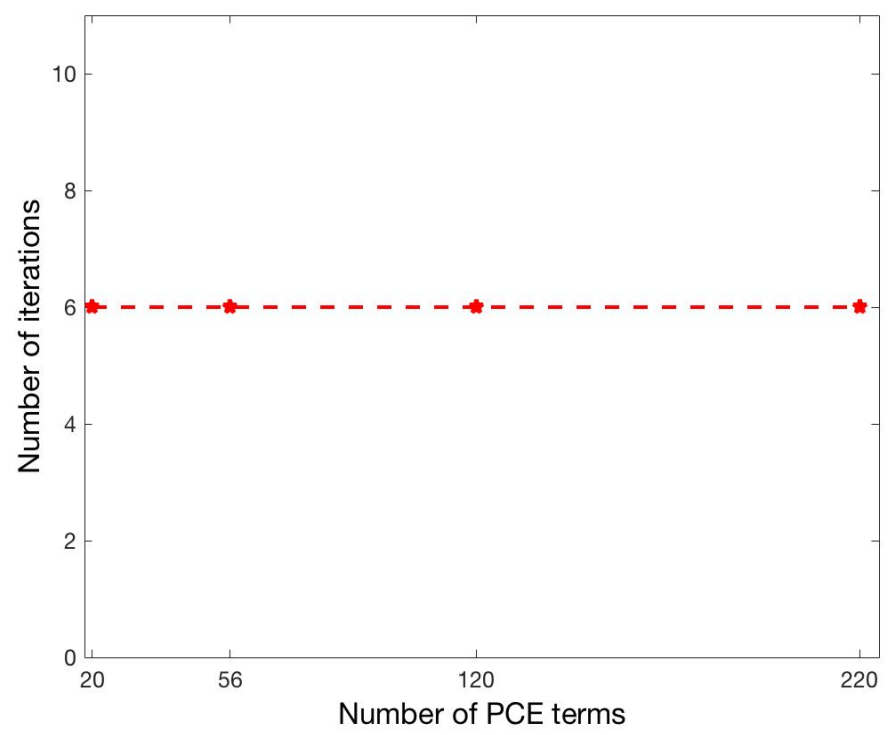

Figure 3.23: Iteration count versus number PCEs for the fixed mesh resolution with fixed number of subdomains.

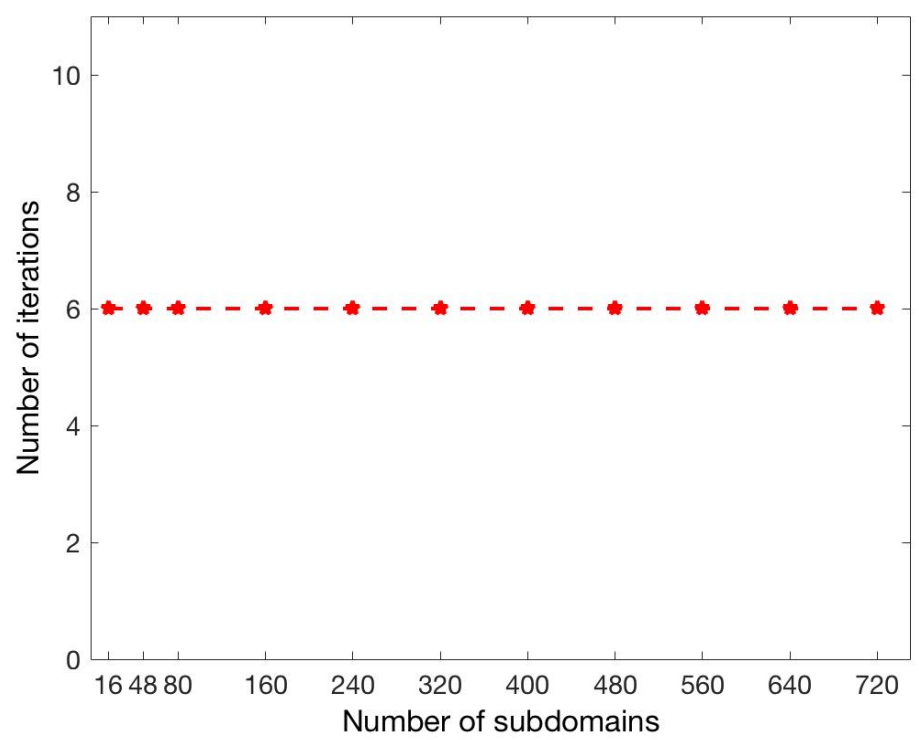

Figure 3.24: Iteration count versus number of subdomains for the fixed mesh resolution with fixed number of PCEs. 


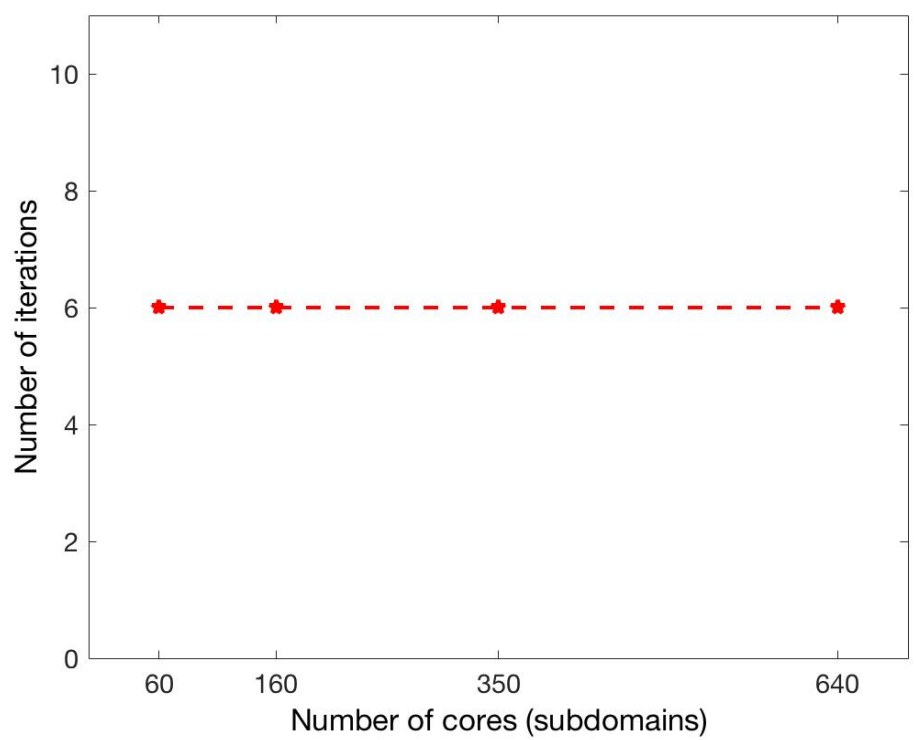

Figure 3.25: Iteration count versus number of subdomains for the fixed mesh resolution with fixed number of PCEs.

\subsubsection{Comparison with Non-Intrusive SSFEM at High Dimensions}

To demonstrate the superiority of intrusive SSFEM equipped with scalable parallel DD solvers, consider a finite element mesh with 52704 nodes and 105410 triangular elements. The intrusive SSFEM systems formulated by using the fixed $p_{u}=3$ and four different cases by selecting the number of RVs as $L=5,10,15$ and 20 are considered. For the parallel solution, we employed BDDC/NNC solver using 80,160,240 and 320 cores respectively. For the same cases, the sparse grid with the level of quadrature, $l=3$ and $l=4$ are employed to solve the problem non-intrusively. Note that the domain decomposition solver is not needed for the non-intrusive approach as each (deterministic) problem for a given sample fits in the memory of a given node. The number of cores employed in the intrusive approach and non-intrusive approach is kept the same, for instance, in the case of $L=5$, we have used 80 cores to solve the intrusive system in parallel. Similarly, we consider that 80 samples can be evaluated in parallel for the nonintrusive approach. Note that, the total CPU time for the non-intrusive approach is computed by multiplying the total number of the samples by the average time required for one sample and then, dividing it by the number of cores. 


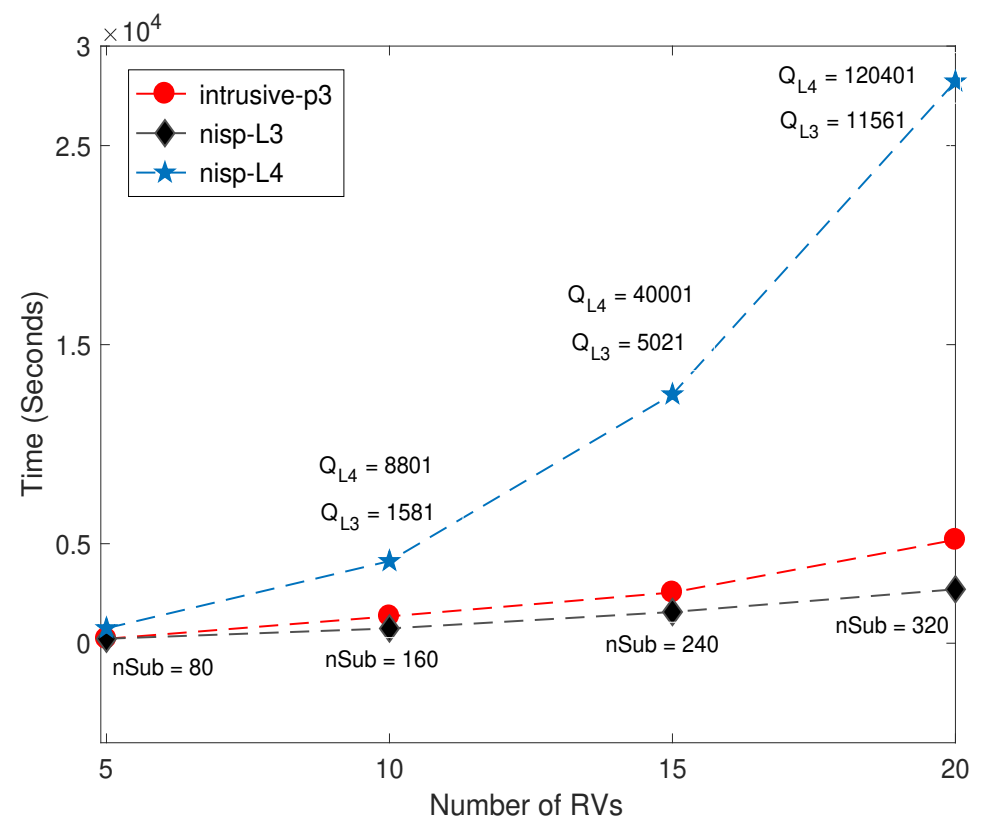

Figure 3.26: Comparison of execution time for intrusive and non-intrusive SSFEM with the fixed mesh resolution and $L=5,10,15,20$.

Computational time for the intrusive and non-intrusive SSFEM for the four different cases by selecting the number of random variables 5, 10, 15 and 20 is plotted in Fig. 3.26. For the thirdlevel sparse grid in non-intrusive approach, the total execution time for all four cases is less compared to that of intrusive approach with the third-order expansion (i.e., $l=p_{u}$ ). However, if we increase the level of sparse grid to 4 (i.e., $l>p_{u}$ ), the computational time required to evaluate the non-intrusive samples rises quickly, especially in the cases with large number of random variables, e.g., 15 or 20. As demonstrated earlier in Fig. 2.11, to achieve the same level of accuracy in PCE coefficients of the solution process we need a higher-level of the sparse grid quadrature in the non-intrusive SSFEM compared to the order of expansion in the intrusive SSFEM (i.e., $l>p_{u}$ ). Therefore, intrusive approach, equipped with the domain decomposition solvers shows computational advantages over non-intrusive approach for the high-dimensional, non-Gaussian stochastic input as considered in this specific application. 
In summary, building on the basic probabilistic formulations of BDDC/NNC [17] and FETIDP [18] solvers for SPDEs, this work extends the algorithms in order to tackle high-dimensional stochastic systems and perform scalability studies with respect to the number of input random variables and the corresponding PCE terms. In contrast to the references $[17,18]$, direct factorization of subdomain-level matrices is avoided and better memory management strategy is implemented for high-dimensional stochastic systems. Firstly, the assembly procedure outlined in Algorithm 2 reduces the memory usage per core and thereby offers the ability to handle higher-dimensional stochastic systems compared to the references [17,18]. Moreover, the local assembly procedure provides a method to utilize existing deterministic FEM packages such as FEniCS, which drastically reduces the coding efforts required for the implementation of the DD-based intrusive SSFEM to a variety of PDEs. Secondly, the third-level iterative solvers used to tackle the subdomain-level local systems reduce the execution time and memory consumption; and hence they offer the ability to tackle stochastic systems with larger number of input random variables and PCE terms than the previous solvers [17, 18]. Finally, we demonstrated the computational superiority of intrusive SSFEM equipped with scalable parallel solvers over non-intrusive SSFEM for non-Gaussian and high-dimensional stochastic systems. These facts highlights the advantages of intrusive approach and demonstrate the necessity of scalable parallel solvers for extreme scale uncertainty quantification. 


\section{Chapter 4}

\section{Domain Decomposition of Stochastic PDEs}

\section{in Three Dimensions: Development of}

\section{Probabilistic Wirebasket Coarse Grid}

A large-scale system of equations arising from the application of domain decomposition algorithms to the problems in three dimensions is computationally more demanding than the problems in two dimensions. This is due to the following reasons: (a) the complex geometry of the global interface and (b) the complicated coupling among the subdomains in three dimensions. For these reasons, the domain decomposition methods such as two-level BDDC/NNC and FETI-DP with the vertex-based coarse grid are inefficient $[128,139]$. The effects are more pronounced for the vector-valued PDEs in three dimensions due to complex spatial coupling among the elements of the solution vector [26, 139, 208]. Moreover, the probabilistic analysis using intrusive PCE adds an additional coupling structure in the resulting system of equations and further complicates the problem $[24,148]$. The coupling among the PCE coefficients of the solution process and the resulting block structure of the stochastic submatrices further influence the condition number of the system resulting in SSFEM [24, 148]. Therefore, the direct application of the Schur complement based two-level domain decomposition algorithms developed 
for two-dimensional stochastic PDEs to three-dimensional problems does not give scalable performance [24].

In the cases of domain decomposition methods for stochastic PDEs in two dimensions discussed earlier in Chapter 3, scalable performance is achieved by providing a mechanism for global communication using vertex-based coarse grid. The coarse problem solved in the vertexbased coarse grid provides the solution only at the corner nodes (vertices), i.e., the nodes shared among three or more subdomains plus the nodes at the ends of interface edge. The solution at the remaining interface nodes, i.e., all other interface nodes excluding vertices, is computed using the available solution at the corner nodes. In three dimensions, this procedure can introduce significant inaccuracy due to an inefficient coarse grid correction resulting from the complicated coupling among the subdomains $[26,139]$.

In the deterministic settings, the condition number bound for the vertex-based method in two dimensions is shown as [26, 128,139]

$$
k \leq C(1+\log (H / h))^{2}
$$

where $C$ is a positive coefficient independent of the size of subdomains $(H)$ and the size of element $(h)$. These bounds are quite satisfactory in two dimensions. However, for the same method in three dimensions, the condition number bound is shown to be $[26,128,139]$

$$
k \leq C(H / h)(1+\log (H / h))
$$

The presence of the additional multiplier $(H / h)$ in the above equation (in contrast to Eq. (4.1)) leads to poor scalability in three-dimensional case.

These issues motivated Bramble [25], Smith [26], Dryja [209] and Mandel [210,211] to work with the wirebasket-based coarse grid as shown in Fig. 4.1 and 4.2. The new coarse grid involves (a) vertices $(\bullet)$, i.e., the nodes shared among three or more subdomains plus the nodes at the ends of interface edge and (b) the remaining nodes on the interface edge i.e., the remaining interface- 
edges (for brevity we omit the word nodes) ( $\star$ ). Together (a) and (b) forms a wirebasket as shown in Fig. 4.1 and 4.2. The interface edge (-) is the boundary of the interface and the nodes on the interface edge (except vertices) are referred as interface-edges. The interface-faces $(\bullet)$ are the nodes shared between two subdomains excluding interface-edges. Note that, the interface in three dimensions is a two-dimensional having a surface defined by interface face and boundary defined by interface edge. The interface in two dimensions is one-dimensional, i.e, an edge (or a line), for instance, see Fig. 3.1.

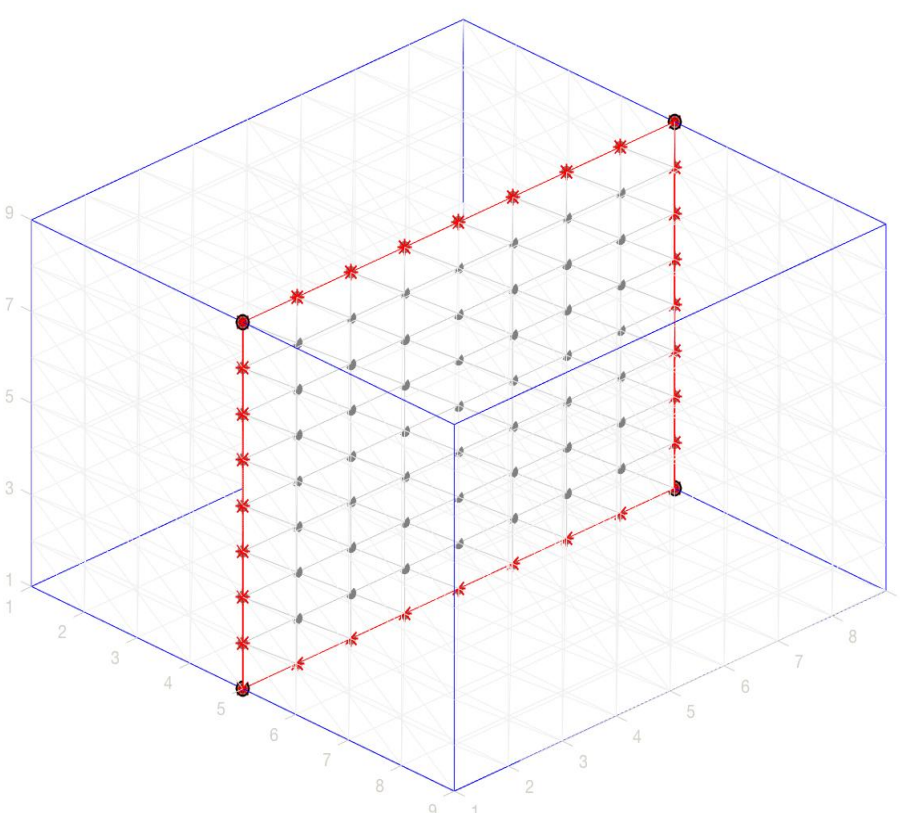

Figure 4.1: Schematic representation of a simple wirebasket coarse grid for a cube partitioned into two subdomains, showing (-) for the global interface edge, $(\bullet)$ as vertices, $(\star)$ as interface-edges and $(\bullet)$ as interface-faces.

The wirebasket-based coarse grid can provide an efficient mechanism for global communication of information, and therefore can help in designing more efficient coarse correction procedure $[26,128,139]$. The condition number bound for the wirebasket-based approach (in the deterministic setting) is given by (refer the following articles and references therein for the theoretical proof $[26,128,139])$, 


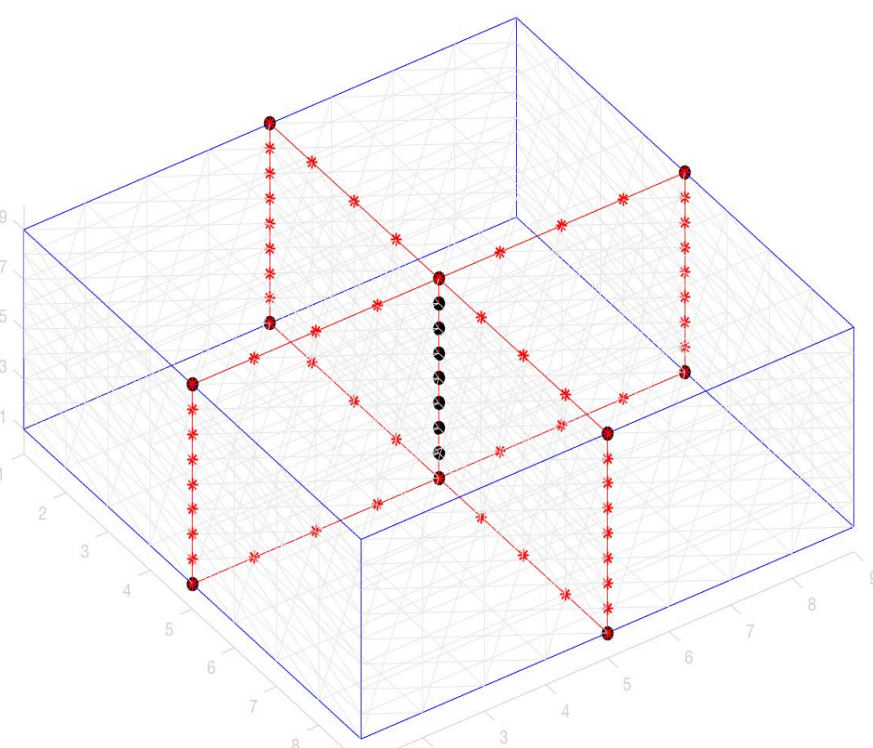

(a)

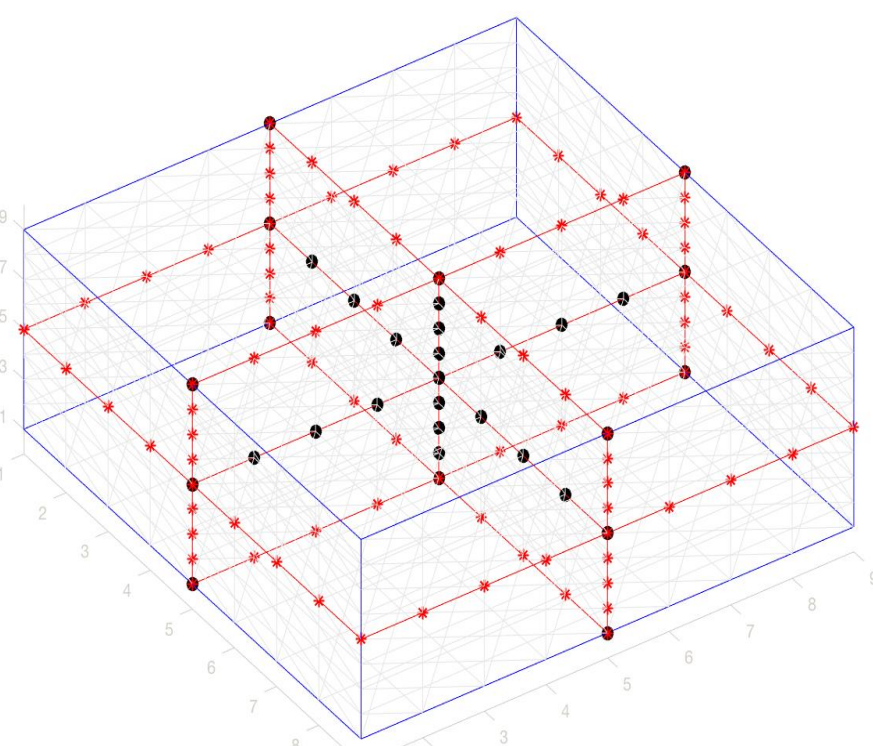

(b)

Figure 4.2: Schematic representation of wirebasket coarse grid for a cube partitioned into (a) four subdomains and (b) eight subdomains, showing (-) for the global interface edge, $(\bullet)$ as vertices and $(\star)$ as interface-edges. 


$$
k \leq C(1+\log (H / h))^{2}
$$

In the context of stochastic PDEs, the direct application of the vertex-based coarse grid to the problems in three dimensions result in poor scaling behavior [24]. This is due to a poorly conditioned system of equations arising in the setting of vertex-based domain decomposition methods and weak mechanism for global error propagation [24]. Moreover, the application of SSFEM further complicates the system due to the coupling among the PCE coefficients of the solution process. This results into block structure of the subdomain-level decomposed matrices, consequently, further affecting the condition number of resulting extended Schur complement system matrix [24].

Taking inspiration from the wirebasket-based approaches developed for the deterministic PDEs in three dimensions [25,26, 128,211], we propose the extended (to the stochastic case) wirebasket-based coarse grid with the BDDC/NNC solver for the stochastic PDEs in three dimensions. The BDDC/NNC solver with the extended wirebasket-based coarse grid is then employed to simulated large-scale, scalar and vector-valued stochastic PDEs with a large number of random variables. To this end, the contributions made in this chapter are broadly categorized next.

- The extended wirebasket-based coarse grid for BDDC/NNC solver is developed to overcome the scalability issues of two-level DD solvers with the vertex-based coarse grid in three dimensions $[24,139]$. The new coarse grid, which includes both vertices and interface-edges to form a wirebasket, strengthens the mechanism for global error propagation and thus improves the convergence. This development enhances the scalability of the BDDC/NNC solver in handling stochastic PDEs in three dimensions.

- The BDDC/NNC solver with the wirebasket-based coarse grid is shown to outperform the vertex-based coarse grid, in regards to the numerical scalability with respect to the number of subdomains and number of random variables. The superiority of the wirebasket-based 
coarse grid is demonstrated for the stochastic diffusion equation and the stochastic equations of linear elasticity in three dimensions.

- Efficient implementational strategies deployed in Chapter 3 are utilized to enhance the capabilities of the wirebasket-based BDDC/NNC solver in order to tackle large-scale stochastic systems with a large number of random variables. The three-level sparse iterative solver which employs an efficient preconditioner at each level is devised and implemented using various sparse data-structures and routines from MPI, PETSc, FEniCS and UQTk [19]. For the cases involving a large number of random variables, an optimal memory management is accomplished via the recursive construction of the subdomain-level sparse blocks of the stochastic system matrices using the temporarily assembled subdomain-level blocks of deterministic system matrices [19].

- Utilizing the subdomain-level stochastic block matrix assembly procedure presented in Algorithm 2, the BDDC/NNC solver with the extended wirebasket-based coarse grid is coupled with the FEniCS deterministic finite element package $[32,177]$. The coupling of BDDC/NNC solver with FEniCS reduces the implementational complexity required in the DD based intrusive SSFEM code development for the three-dimensional stochastic PDEs [9, 106, 108].

- To simplify the application of intrusive SSFEM for a coupled stochastic PDE system (such as equations of linear elasticity), the formulation of extended Schur complement system for coupled stochastic PDE system is presented. Efforts have been made to clarify the couplings structure in the linear system arising in the intrusive SSFEM. The first-level of coupling is due to vector-valued solution process of the coupled PDE system and the second-level of coupling is due to the interdependence of PCE coefficients of the solution process.

In this thesis, no attempts have been made to perform the theoretical convergence analysis and condition number bound estimation of the preconditioned extended Schur complement system in the intrusive SSFEM setting. However, extensive numerical experiments are 
conducted to thoroughly investigate the performance of the extended wirebasket-based BDDC/NNC solver. Modern HPC clusters (available in compute Canada [205]) are exploited to demonstrate the utility of BDDC/NNC solver with the wirebasket-based coarse grid to tackle large-scale discretized stochastic PDEs with a large number of random variables.

First, the numerical scalability concerning the number of PCGM iterations to solve the extended Schur complement system is investigated with respect to the following: (a) increasing spatial mesh resolution with a fixed number of PCE terms, (b) increasing number of subdomains with increasing number of PCE terms for a fixed problem size per subdomains, (c) increasing number of subdomains for a fixed mesh resolution and a fixed number of PCE terms, (d) increasing number of random variables for a fixed mesh resolution and a fixed order of expansion, and (e) increasing order of expansion for a fixed mesh resolution and a fixed number of random variables.

Secondly, the parallel scalability of the extended wirebasket-based BDDC/NNC solver is measured concerning the strong and weak scaling [121,139]. In the strong scalability test, the global problem size is kept constant and the number of cores used to solve the problem is increased to reduce the total execution time. For the weak scalability test, the problem size per subdomain is fixed and the global size of the problem is increased by adding more subdomains. In the weak scaling, the goal is to solve a larger global problem using proportionally more cores in nearly a constant execution time [121,139].

Finally, the scalability study of the wirebasket-based BDDC/NNC solver concerning stochastic parameters such as the number of random variables and order of expansion is presented. The scalability of the solver with respect to stochastic parameters is crucial for uncertainty quantification. That is because, as the number of PCE terms increases, the block-sparsity structure of the underlying intrusive SSFEM system matrix changes. These effects are more substantial in the cases of the three-dimensional coupled PDE system due to the additional coupling in the spatial domain. Therefore, the scalability plots concerning stochastic parameters provide useful information regarding the usefulness of the solver for large-scale stochastic simulations. 
The scalability study concerning stochastic parameters is somewhat unusual compared to the scalability studies commonly conducted for the deterministic FEM. That is because, in the deterministic setting, changing mesh density or the values of the system parameters, does not influence the block sparsity pattern of the underlying system matrix.

In the following sections, we first discuss the formulation of extended Schur complement of a coupled stochastic PDE system in Sec. 4.1. Next the formulation of extended wirebasket-based BDDC/NNC preconditioner for coupled stochastic PDE system is presented in Sec. 4.2. This is followed by the application of extended wirebasket-based BDDC/NNC solver to the threedimensional flow through random media modeled by using steady-state diffusion equation in Sec. 4.3. The application of BDDC/NNC solver with the extended wirebasket-based coarse grid to a small elastic deformation of a three-dimensional body modeled by using equations of linear elasticity with random Young's modulus is presented in Sec. 4.4. For both stochastic Poisson and elasticity problems, the random system parameters are modeled as non-Gaussian stochastic processes characterized by using a large number of RVs (up to $15 \mathrm{RVs}$ ).

\subsection{Extended Schur Complement of Coupled Stochastic PDE}

\section{System}

In this section, we formulate an extended Schur complement system for a coupled stochastic PDE system. For demonstration we consider the equations of linear elasticity in threedimensions to model the vector-valued stochastic displacement field. The finite element discretization of a coupled stochastic elliptic PDE system defined over physical domain $\mathcal{D}(x, y, z)$ as a function of random event $\theta$, yields the following stochastic linear system,

$$
\mathbf{A}(\theta) \mathbf{u}(\theta)=\mathbf{f}
$$


where $\mathbf{A}(\theta)$ is a coupled stochastic intrusive system matrix. The vector-valued stochastic response field $\mathbf{u}(\theta)$ can be denoted as,

$$
\mathbf{u}(\theta)=\left\{\mathbf{u}_{x}(\theta), \mathbf{u}_{y}(\theta), \mathbf{u}_{z}(\theta)\right\}
$$

For simplicity, the right hand side vector $\mathbf{f}$ is considered to be a deterministic source term. However, the methodology presented here can be easily extended to the stochastic source term [1,2].

To formulate the Schur complement system, we divide the domain $\mathcal{D}$ into $n_{s}$ non-overlapping subdomains with $s=1,2, \ldots, n_{s}$. Accordingly, the subdomain-level equilibrium equation for a typical subdomain $s$ can be written as [19,24],

$$
\mathbf{A}^{s}(\theta) \mathbf{u}^{s}(\theta)=\mathbf{f}^{s}
$$

The local system matrix $\mathbf{A}^{s}(\theta)$ can be expressed in terms of the contribution from $x, y$ and $z$ coordinates. Therefore, the coupled subdomain-level stochastic equilibrium system can be expanded as

$$
\left[\begin{array}{ccc}
\mathbf{A}_{x x}^{s}(\theta) & \mathbf{A}_{x y}^{s}(\theta) & \mathbf{A}_{x z}^{s}(\theta) \\
\mathbf{A}_{y x}^{s}(\theta) & \mathbf{A}_{y y}^{s}(\theta) & \mathbf{A}_{y z}^{s}(\theta) \\
\mathbf{A}_{z x}^{s}(\theta) & \mathbf{A}_{z y}^{s}(\theta) & \mathbf{A}_{z z}^{s}(\theta)
\end{array}\right]\left\{\begin{array}{l}
\mathbf{u}_{x}^{s}(\theta) \\
\mathbf{u}_{y}^{s}(\theta) \\
\mathbf{u}_{z}^{s}(\theta)
\end{array}\right\}=\left\{\begin{array}{l}
\mathbf{f}_{x}^{s} \\
\mathbf{f}_{y}^{s} \\
\mathbf{f}_{z}^{s}
\end{array}\right\} .
$$

The sparsity structure of the subdomain-level block matrix $\mathbf{A}^{s}(\theta)$ in Eq. (4.6) for a typical subdomain $s$, assembled at the mean values of the stochastic system parameters for the equations of linear elasticity is shown in Fig. 4.3.

The stochastic solution vector $\mathbf{u}^{s}(\theta)$ for each subdomain $s$, has three components $\mathbf{u}_{x}^{s}(\theta)$, $\mathbf{u}_{y}^{s}(\theta), \mathbf{u}_{z}^{s}(\theta)$ along spatial coordinates $x, y, z$ respectively. Let us denote the submatrix and the subvector components from Eq. (4.6) using $\mathbf{A}_{\alpha \beta}^{s}(\theta)$ and $\mathbf{u}_{\alpha}^{s}(\theta)$, where the $\alpha$ and $\beta$ represent $x, y$ and $z$ components. Next, the solution vector components $\mathbf{u}_{\alpha}^{s}(\theta)$ are divided into the in- 


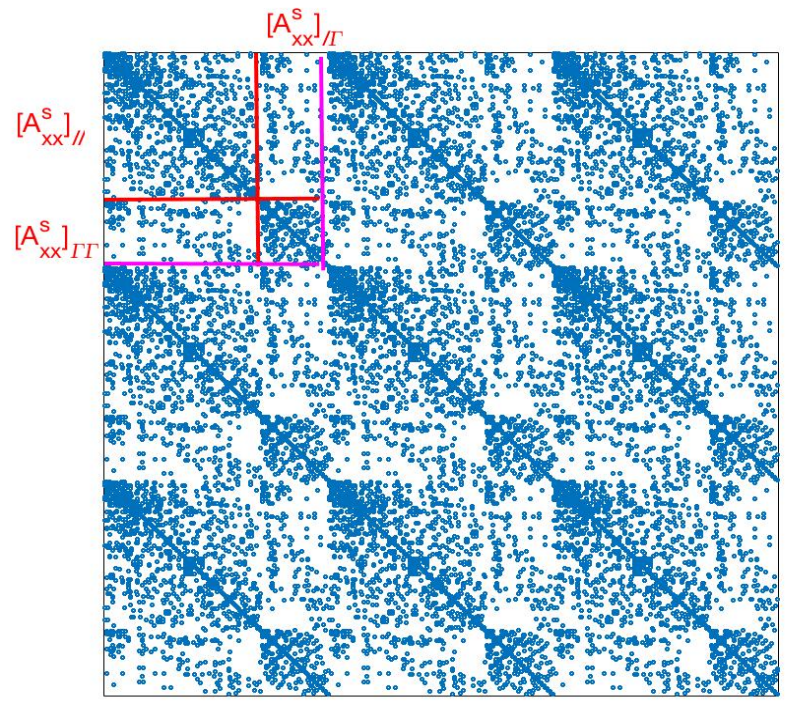

Figure 4.3: Subdomain-level system matrix $\mathbf{A}^{s}(\theta)$ computed using the mean system parameters, indicating coupling among $x, y$ and $z$ displacements. Also displays decomposed components for $x$ displacements according to interior $I$ and interface $\Gamma$ nodes.

terior vector $\left(\mathbf{u}_{\alpha}^{s}\right)_{I}(\theta)$ and interface vector $\left(\mathbf{u}_{\alpha}^{s}\right)_{\Gamma}(\theta)$. According to this decomposition, each submatrix and subvector in Eq. (4.6) can be written as

$$
\begin{gathered}
\mathbf{A}_{\alpha \beta}^{s}=\left[\begin{array}{ll}
\left(\mathbf{A}_{\alpha \beta}^{s}\right)_{I I}(\theta) & \left(\mathbf{A}_{\alpha \beta}^{s}\right)_{I \Gamma}(\theta) \\
\left(\mathbf{A}_{\alpha \beta}^{s}\right)_{\Gamma I}(\theta) & \left(\mathbf{A}_{\alpha \beta}^{s}\right)_{\Gamma \Gamma}(\theta)
\end{array}\right] \\
\mathbf{u}_{\alpha}^{s}(\theta)=\left[\left(\mathbf{u}_{\alpha}^{s}\right)_{I}(\theta),\left(\mathbf{u}_{\alpha}^{s}\right)_{\Gamma}(\theta)\right]^{\mathrm{T}}
\end{gathered}
$$

For example, see Fig. 4.3 which displays the decomposed matrix components $\left(\mathbf{A}_{\alpha \beta}^{s}\right)_{\gamma \delta}(\theta)$, according to interior and interface nodes for $\mathbf{A}_{x x}^{s}(\theta)$ matrix where $\gamma$ and $\delta$ represent $I$ and $\Gamma$.

Gathering, for example, all components of decomposed matrices and vectors which relate to 


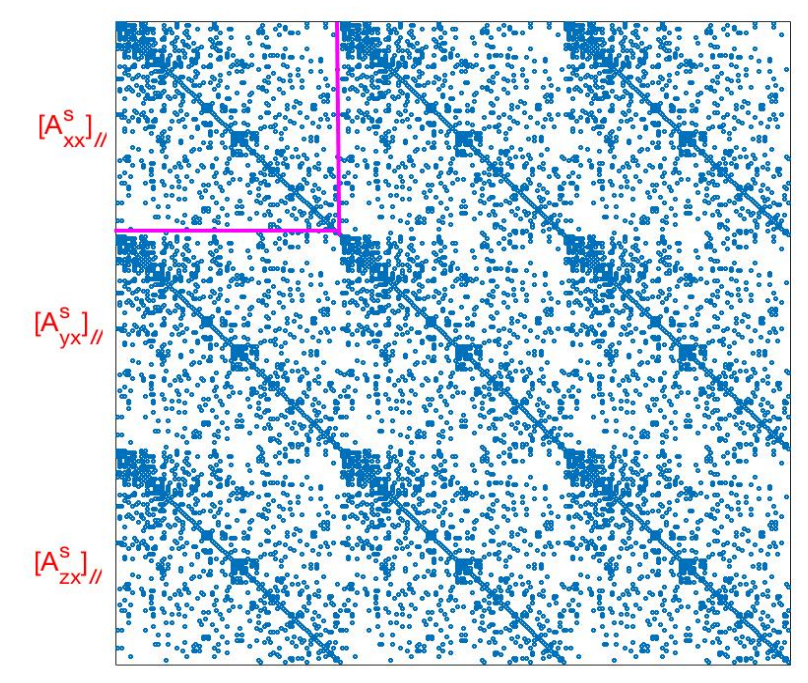

Figure 4.4: Subdomain-level stiffness matrix $\boldsymbol{A}_{I I}^{s}(\theta)$ for interior nodes, computed using the mean system parameters, indicating coupling among $x, y$ and $z$ displacements.

only interior nodes we get,

$$
\begin{gathered}
\boldsymbol{A}_{I I}^{s}(\theta)=\left[\begin{array}{lll}
\left(\mathbf{A}_{x x}^{s}\right)_{I I}(\theta) & \left(\mathbf{A}_{x y}^{s}\right)_{I I}(\theta) & \left(\mathbf{A}_{x z}^{s}\right)_{I I}(\theta) \\
\left(\mathbf{A}_{y x}^{s}\right)_{I I}(\theta) & \left(\mathbf{A}_{y y}^{s}\right)_{I I}(\theta) & \left(\mathbf{A}_{y z}^{s}\right)_{I I}(\theta) \\
\left(\mathbf{A}_{z x}^{s}\right)_{I I}(\theta) & \left(\mathbf{A}_{z y}^{s}\right)_{I I}(\theta) & \left(\mathbf{A}_{z z}^{s}\right)_{I I}(\theta)
\end{array}\right] \\
\boldsymbol{u}_{I}^{s}(\theta)=\left[\left(\mathbf{u}_{x}^{s}\right)_{I}(\theta),\left[\left(\mathbf{u}_{y}^{s}\right)_{I}(\theta),\left[\left(\mathbf{u}_{z}^{s}\right)_{I}(\theta)\right]^{\mathrm{T}} .\right.\right.
\end{gathered}
$$

For example, see Fig. 4.4 which displays all the gathered blocks pertains to interior nodes for the matrix $\boldsymbol{A}_{I I}^{s}(\theta)$ as in Eq. (4.9).

Similar step can be taken to construct the other component matrices $\left(\mathbf{A}_{\alpha \beta}^{s}\right)_{\gamma \delta}(\theta)$ and vectors $\left(\mathbf{u}_{\alpha}^{s}\right)_{\gamma}(\theta)$. Consequently, the subdomain-level equilibrium equation for a typical subdomain $s$ can be obtained by using rearranged component matrices and vectors as,

$$
\left[\begin{array}{cc}
\boldsymbol{A}_{I I}^{s}(\theta) & \boldsymbol{A}_{I \Gamma}^{s}(\theta) \\
\boldsymbol{A}_{\Gamma I}^{s}(\theta) & \boldsymbol{A}_{\Gamma \Gamma}^{s}(\theta)
\end{array}\right]\left\{\begin{array}{l}
\boldsymbol{u}_{I}^{s}(\theta) \\
\boldsymbol{u}_{\Gamma}^{s}(\theta)
\end{array}\right\}=\left\{\begin{array}{l}
\boldsymbol{f}_{I}^{s} \\
\boldsymbol{f}_{\Gamma}^{s}
\end{array}\right\}
$$


where $\boldsymbol{u}_{I}^{s}(\theta)$ is the subdomain-level (local) interior solution vector relating to the interior nodes and $\boldsymbol{u}_{\Gamma}^{s}(\theta)$ is the local interface solution vector pertaining to interface nodes. The $\boldsymbol{f}_{I}^{s}$ and $\boldsymbol{f}_{\Gamma}^{s}$ are corresponding source vectors for interior and interface, respectively.

Next, by following these steps the extended (to the stochastic case) Schur complement system is obtained as follows:

- Incorporate PCE of the stochastic system matrix blocks, $\boldsymbol{A}_{\gamma \delta}^{s}(\theta) \approx \sum_{i=0}^{P_{\mathrm{A}}} \overline{\boldsymbol{A}}_{\gamma \delta, i}^{s} \Psi_{i}(\theta)$, using $P_{\mathrm{A}}$ terms in the expansion and the stochastic solution process, $\boldsymbol{u}_{\gamma}^{s}(\theta) \approx \sum_{j=0}^{P_{u}} \overline{\boldsymbol{u}}_{\gamma, j}^{s} \Psi_{j}(\theta)$ using $P_{u}$ terms in the expansion, into Eq. (4.11).

- This is followed by Galerkin projection along random dimension, i.e., multiply both sides of modified Eq. (4.11) by $\Psi_{k}(\theta)$ with $k=\left\{0,1, \ldots P_{u}\right\}$ and take the expectation of both sides.

- Enforcing the transmission conditions by performing global assembly along the interface unknowns and then using Gaussian elimination to eliminate interior degrees of freedom from resulting equation leads to the extended Schur complement system (refer to the following articles for further details $[19,24])$, yielding

$$
\mathcal{S} \mathcal{U}_{\Gamma}=\boldsymbol{g}_{\Gamma}
$$

where $\mathcal{S}$ is the extended Schur complement matrix for a coupled stochastic PDE system and $\boldsymbol{g}_{\Gamma}$ is the corresponding right hand side vector. They are respectively defined as

$$
\begin{aligned}
\mathcal{S} & =\sum_{s=1}^{n_{s}} \boldsymbol{\mathcal { R }}_{s}^{\mathrm{T}}\left[\mathcal{A}_{\Gamma \Gamma}^{s}-\mathcal{A}_{\Gamma I}^{s}\left[\mathcal{A}_{I I}^{s}\right]^{-1} \mathcal{A}_{I \Gamma}^{s}\right] \mathcal{R}_{s}, \\
\boldsymbol{g}_{\Gamma} & =\sum_{s=1}^{n_{s}} \boldsymbol{\mathcal { R }}_{s}^{\mathrm{T}}\left[\mathcal{F}_{\Gamma}^{s}-\mathcal{A}_{\Gamma I}^{s}\left[\mathcal{A}_{I I}^{s}\right]^{-1} \mathcal{F}_{I}^{s}\right]
\end{aligned}
$$


where the $\mathcal{R}_{s}$ are the restriction matrices defined later and

$$
\begin{aligned}
{\left[\mathcal{A}_{\gamma \delta}^{s}\right]_{j k} } & =\sum_{i=0}^{P_{\mathrm{A}}}\left\langle\psi_{i} \psi_{j} \psi_{k}\right\rangle \overline{\boldsymbol{A}}_{\gamma \delta, i}^{s}, \\
\mathcal{U}_{\Gamma} & =\left[\overline{\boldsymbol{u}}_{\Gamma, 0} \ldots, \overline{\boldsymbol{u}}_{\Gamma, P_{u}}\right]^{\mathrm{T}}, \\
\mathcal{F}_{\gamma, k}^{s} & =\left\langle\psi_{k} \boldsymbol{f}_{\gamma}^{s}\right\rangle .
\end{aligned}
$$

The subdomain-level local matrices $\left[\mathcal{A}_{\gamma \delta}^{s}\right]_{j k}$ can be assembled by using the stochastic finite element matrix assembly procedure outlined in Algorithm 2. This procedure utilizes deterministic FEniCS assembly routines, which involve manipulating element stiffness matrices to handle stochasticity (see Appendix B.2 for implementational details). The structure of the local matrices $\left[\mathcal{A}_{\gamma \delta}^{s}\right]$ have two levels of blocks. The first-level of blocks is due to couplings among $x, y$ and $z$ components and the second-level of blocks is due to couplings among the PCE coefficients. These couplings produce two-levels of block-sparsity structure in each of the subdomain level decomposed matrices $\left[\mathcal{A}_{\gamma \delta}^{s}\right]$.

The restriction operators $\boldsymbol{\mathcal { R }}_{s}$ in Eq. (4.13) and Eq. (4.14) can be obtained as

$$
\boldsymbol{\mathcal { R }}_{s}=\operatorname{blockdiagonal}\left(\boldsymbol{R}_{s, 0}, \ldots, \boldsymbol{R}_{s, P_{u}}\right)
$$

where each of the blocks (with $i=\left\{0,1, \ldots, P_{u}\right\}$ ) can be obtained as

$$
\boldsymbol{R}_{s, i}=\operatorname{blockdiagonal}\left(\mathbf{R}_{s}^{x, i}, \mathbf{R}_{s}^{y, i}, \mathbf{R}_{s}^{z, i}\right)
$$

The $\mathbf{R}_{s}^{\alpha, i}$ in eq. (4.19), where $\alpha$ denotes $x, y$ or $z$, acts as a scatter or gather operator between global and local components of the deterministic interface solution vector as follows,

$$
\begin{aligned}
& \left(\mathbf{u}_{\alpha, i}^{s}\right)_{\Gamma}=\left[\mathbf{R}_{s}^{\alpha, i}\right]\left(\mathbf{u}_{\alpha, i}\right)_{\Gamma}, \\
& \left(\mathbf{u}_{\alpha, i}\right)_{\Gamma}=\left[\mathbf{R}_{s}^{\alpha, i}\right]^{\mathrm{T}}\left(\mathbf{u}_{\alpha, i}^{s}\right)_{\Gamma} .
\end{aligned}
$$


The matrix components, $\mathcal{A}_{\gamma \delta}^{s}$ in Eq. (4.13) and (4.14) with $\gamma$ and $\delta$ representing $I$ or $\Gamma$, are considerably larger, complex and computationally intensive to assemble compared to $A_{\gamma \delta}^{s}$ for scalar-valued stochastic PDEs (see Sec. 3.1.1 in Chapter 3). Therefore, to simplify assembly procedure for stochastic matrix components $\mathcal{A}_{\gamma \delta}^{s}$, FEniCS-based deterministic assembly routines are employed (for example, see Algorithm 12). Similarly the restriction operators $\boldsymbol{\mathcal { R }}_{s}$ for PDE system are also expensive to obtained compared to $R_{s}$ for scalar-valued stochastic PDEs.

The probabilistic Schur complement system in Eq. (4.12) for a stochastic PDE system is more complex and computationally expensive compared to the extended Schur complement system obtained in the case of scalar-valued stochastic PDEs (see Sec. 3.1.1 in Chapter 3). The solution of extended Schur complement system using two-level preconditioner with vertexbased coarse grid does not give scalable performance [24]. Therefore, the wirebasket-based coarse grid is required to overcome this challenge. The probabilistic two-level preconditioner using extended wirebasket for stochastic PDE system is presented in the subsequent section.

\subsection{Two-Level Preconditioner using Extended Wirebasket- Based Coarse Grid}

Two-level preconditioner with a coarse problem is required to provide a mechanism for a global transfer of information, which is essential to achieve scalable performance with DD-based iterative solvers $[24,101]$. To overcome the scalability issues of vertex-based coarse grid, we propose extended wirebasket-based coarse grid with the two-level BDDC/NNC preconditioner.

The wirebasket, as shown in Fig. 4.1, includes the interface edges (nodes on the edges of the boundary $\star$ ) along with the vertices (nodes shared by three or more subdomains plus the nodes at the end of the interface $\bullet$ ). The wirebasket can provides an efficient mechanism for global error propagation during each iteration of BDDC/NNC based PCGM solver of the extended Schur complement system shown in Eq. (4.12). The interface-faces •, i.e., the nodes shared by two subdomains (excluding the interface-edges) are also showed in Fig. 4.1. The formulation 
of extended wirebasket-based BDDC/NNC preconditioner is briefly discussed below.

The local interface unknowns $\mathcal{U}_{\Gamma}^{s}$ (for each subdomain $s$ ) are partitioned into faces $\mathcal{U}_{F}^{s}$ and wirebasket $\mathcal{U}_{W}^{s}$ such that

$$
\left\{\begin{array}{c}
\mathcal{U}_{F}^{s} \\
\mathcal{U}_{W}^{s}
\end{array}\right\}=\left\{\begin{array}{c}
\mathcal{R}_{s}^{F} \\
\mathcal{R}_{s}^{W}
\end{array}\right\} \mathcal{U}_{\Gamma}^{s}
$$

where the restriction operators are obtained as,

$$
\begin{gathered}
\mathcal{R}_{s}^{F}=\operatorname{blockdiagonal}\left(\boldsymbol{R}_{s, 0}^{F}, \ldots, \boldsymbol{R}_{s, P_{u}}^{F}\right), \\
\boldsymbol{\mathcal { R }}_{s}^{W}=\operatorname{blockdiagonal}\left(\boldsymbol{R}_{s, 0}^{W}, \ldots, \boldsymbol{R}_{s, P_{u}}^{W}\right) .
\end{gathered}
$$

The $P_{u}$ is the number of PCE terms used in the characterization of solution process. The $\boldsymbol{R}_{s, i}^{\gamma}$ blocks in Eq. (4.23) and (4.24) where $\gamma$ represents $F$ or $W$ and $i=\left\{0,1, \ldots, P_{u}\right\}$ are represented by the component matrices as follows:

$$
\begin{aligned}
& \boldsymbol{R}_{s, i}^{F}=\operatorname{blockdiagonal}\left(\mathbf{R}_{s, i}^{F x}, \mathbf{R}_{s, i}^{F y}, \mathbf{R}_{s, i}^{F z}\right) \\
& \boldsymbol{R}_{s, i}^{W}=\operatorname{blockdiagonal}\left(\mathbf{R}_{s, i}^{W x}, \mathbf{R}_{s, i}^{W y}, \mathbf{R}_{s, i}^{W z}\right)
\end{aligned}
$$

The components $\mathbf{R}_{s, i}^{\gamma \delta}$ (with $\delta$ represents $x, y$ or $z$ ), acts as a scatter or gather operator between global faces/wirebasket and local faces/wirebasket components of the deterministic interface solution vectors.

Accordingly the global coarse problem arising in the setting of BDDC/NNC preconditioner is (refer to $[19,24]$ for detailed procedure to construct coarse problem),

$$
\mathcal{F}_{W W} \mathcal{U}_{W}=d_{W}
$$

where $\mathcal{U}_{W}$ denotes global wirebasket unknowns, $\mathcal{F}_{W W}$ is the coarse operator and $d_{W}$ is corre- 
sponding right-hand side vector.

The $\mathcal{F}_{W W}$ and $d_{W}$ are obtained as,

$$
\begin{aligned}
\mathcal{F}_{W W} & =\sum_{s=1}^{n_{s}} \mathcal{B}_{W}^{s} \mathrm{~T}\left(\mathcal{S}_{W W}^{s}-\mathcal{S}_{W F}^{s}\left[\mathcal{S}_{F F}^{s}\right]^{-1} \mathcal{S}_{F W}^{s}\right) \mathcal{B}_{W}^{s} \\
d_{W} & =\sum_{s=1}^{n_{s}} \mathcal{B}_{W}^{s} \mathrm{~T}\left(f_{W}^{s}-\mathcal{S}_{W F}^{s}\left[\mathcal{S}_{F F}^{s}\right]^{-1} f_{F}^{s}\right)
\end{aligned}
$$

where $\mathcal{S}_{\alpha \beta}^{s}=\mathcal{A}_{\alpha \beta}^{s}-\mathcal{A}_{\alpha I}^{s}\left[\mathcal{A}_{I I}^{s}\right]^{-1} \mathcal{A}_{I \beta}^{s}$, where $\alpha$ and $\beta$ represent $W$ or $F$.

The $\mathcal{B}_{W}^{s}$ is the restriction operator that maps the global wirebasket unknowns to local wirebasket unknowns. The $\mathcal{B}_{W}^{s}$ can be obtained as,

$$
\mathcal{B}_{W}^{s}=\operatorname{blockdiagonal}\left(\boldsymbol{B}_{W, 0}^{s}, \ldots, \boldsymbol{B}_{W, P_{u}}^{s}\right)
$$

where each of the block (with $i=\left\{0,1, \ldots, P_{u}\right\}$ ) can be obtained as

$$
\boldsymbol{B}_{W, i}^{s}=\operatorname{blockdiagonal}\left(\mathbf{B}_{W, x, i}^{s}, \mathbf{B}_{W, y, i}^{s}, \mathbf{B}_{W, z, i}^{s}\right)
$$

and the deterministic restriction operator $\mathbf{B}_{W, \alpha, i}^{s}$ maps the global corner node solution vector into local corner node output as:

$$
\left(\mathbf{u}_{\alpha, i}^{s}\right)_{W}=\left[\mathbf{B}_{W, \alpha, i}^{s}\right]\left(\mathbf{u}_{\alpha, i}\right)_{W}
$$

where $\alpha$ represents $x, y$ and $z$.

Performing algebraic manipulations, the two-level BDDC/NNC preconditioner with the wirebasketbased coarse grid can be written as (refer [19,24] for further details),

$$
\mathcal{M}_{N N W}^{-1}=\sum_{s=1}^{n_{s}} \boldsymbol{\mathcal { R }}_{s}^{\mathrm{T}} \mathcal{D}_{s}\left(\boldsymbol{\mathcal { R }}_{s}^{F^{\mathrm{T}}}\left[\mathcal{S}_{F F}^{s}\right]^{-1} \boldsymbol{\mathcal { R }}_{s}^{F}\right) \mathcal{D}_{s} \boldsymbol{\mathcal { R }}_{s}+\boldsymbol{\mathcal { R }}_{0}^{\mathrm{T}}\left[\mathcal{F}_{W W}\right]^{-1} \boldsymbol{\mathcal { R }}_{0}
$$

The $\boldsymbol{\mathcal { R }}_{0}$ in Eq. (4.33) acts as a mapping operator between global fine grid and coarse grid 
components [19,24], as given by

$$
\mathcal{R}_{0}=\sum_{s=1}^{n_{s}} \mathcal{B}_{W}^{s}{ }^{\mathrm{T}}\left(\mathcal{R}_{s}^{W}-\mathcal{S}_{W F}^{s}\left[\mathcal{S}_{F F}^{s}\right]^{-1} \mathcal{R}_{s}^{F}\right) \mathcal{D}_{s} \mathcal{R}_{s}
$$

where $\mathcal{D}_{s}$ is a diagonal scaling operator obtained as follows,

$$
\mathcal{D}_{s}=\operatorname{blockdiagonal}\left(\boldsymbol{D}_{s, 0}, \ldots, \boldsymbol{D}_{s, P_{u}}\right)
$$

where $\boldsymbol{D}_{s, i}$ are obtained by using deterministic diagonal scaling matrices $\mathbf{D}_{s, i}^{\alpha}$ as,

$$
\boldsymbol{D}_{s, i}=\operatorname{blockdiagonal}\left(\mathbf{D}_{s, i}^{x}, \mathbf{D}_{s, i}^{y}, \mathbf{D}_{s, i}^{z}\right)
$$

where $i=\left\{0,1, \ldots, P_{u}\right\}$ and $\alpha$ represent $x, y$ and $z$.

From Eq. (4.33), it can be noted that the extended wirebasket-based BDDC/NNC preconditioner consists of two operators. First, the local-fine operator $\left(\mathcal{R}_{s}^{F^{\mathrm{T}}}\left[\mathcal{S}_{F F}^{s}\right]^{-1} \boldsymbol{\mathcal { R }}_{s}^{F}\right)$, which consists of solving one local fine problem $\left[\mathcal{S}_{F F}^{s}\right]^{-1}$ on each of the subdomains for each PCGM iteration, concerning interface-faces nodes. Second, the global coarse operator $\boldsymbol{\mathcal { R }}_{0}^{\mathrm{T}}\left[\mathcal{F}_{W W}\right]^{-1} \boldsymbol{\mathcal { R }}_{0}$, which consists of solving a global coarse problem $\left[\mathcal{F}_{W W}\right]^{-1}$ for each PCGM iteration, concerning the wirebasket nodes. This coarse operator provides an efficient mechanism for global error propagation during each application of preconditioner, therefore, accelerates the convergence of the PCGM solver [26, 139, 200].

In this following sections, we utilize the extended wirebasket-based coarse grid presented in this section to solve both scalar and vector-valued stochastic PDEs in three-dimensions. For an exposition of the methodology, we first consider steady-state stochastic diffusion equation in three-dimensions to model the scalar-valued stochastic solution process. This is followed by the equations of linear elasticity in three-dimensions to model the vector-valued stochastic displacement field. 


\subsection{Three-Dimensional Poisson Problem}

Consider a three-dimensional steady-state flow through random media with a spatially varying non-Gaussian diffusion coefficient. The flow is modeled by a three-dimensional stochastic diffusion equation. This leads to a Poisson problem defined by a linear elliptic stochastic PDE on a spatial domain $\mathscr{D}$ with a Dirichlet boundary condition as defined below:

$$
\begin{aligned}
-\nabla \cdot\left(c_{d}(\boldsymbol{x}, \theta) \nabla \mathcal{U}(\boldsymbol{x}, \theta)\right) & =F(\boldsymbol{x}), & & \mathscr{D} \times \Omega, \\
\mathcal{U}(\boldsymbol{x}, \theta) & =0, & & \delta \mathscr{D} \times \Omega,
\end{aligned}
$$

where $\nabla$ is the differential operator with respect to the spatial variables $\boldsymbol{x}=\{x, y, z\}, F(\boldsymbol{x})$ is the deterministic forcing term, $\mathscr{U}$ is the solution process, $\theta$ is an element in the sample space $\Omega$ defined by the complete probability space $(\Omega, \mathscr{E}, \mathscr{P})[181]$.

The diffusion coefficient $c_{d}$ is modeled as a lognormal stochastic process, $l(\boldsymbol{x}, \theta)=\exp (g(\boldsymbol{x}, \theta))$ with the underlying Gaussian process $g(\boldsymbol{x}, \theta)$ having a mean $\mu$, variance $\sigma^{2}$ and the exponential covariance function $C$ defined as [1,24],

$$
C\left(x_{1}, y_{1}, z_{1} ; x_{2}, y_{2}, z_{2}\right)=\sigma^{2} e^{-\left|x_{2}-x_{1}\right| / b_{x}-\left|y_{2}-y_{1}\right| / b_{y}-\left|z_{2}-z_{1}\right| / b_{z}},
$$

where $b_{x}, b_{y}$ and $b_{z}$ are the correlation lengths along $x, y$ and $z$ directions respectively.

For simplicity, we consider deterministic source term $F(\boldsymbol{x})=1$. The correlation lengths $b_{x}=$ $b_{y}=b_{z}=1$ and standard deviation $\sigma=0.3$ are used to characterize the underlying Gaussian process. Numerical experiments are performed for the three-dimensional computational domain shown in Fig. 4.5. A typical unstructured finite element mesh with 31598 nodes and 182681 linear (four node) tetrahedral elements partitioned into 320 subdomains is displayed in Fig. 4.5.

Similar to the two-dimensional cases, the primary focus is given to investigate the performance of the solvers to handle the high-dimensional stochastic systems. Therefore, various 


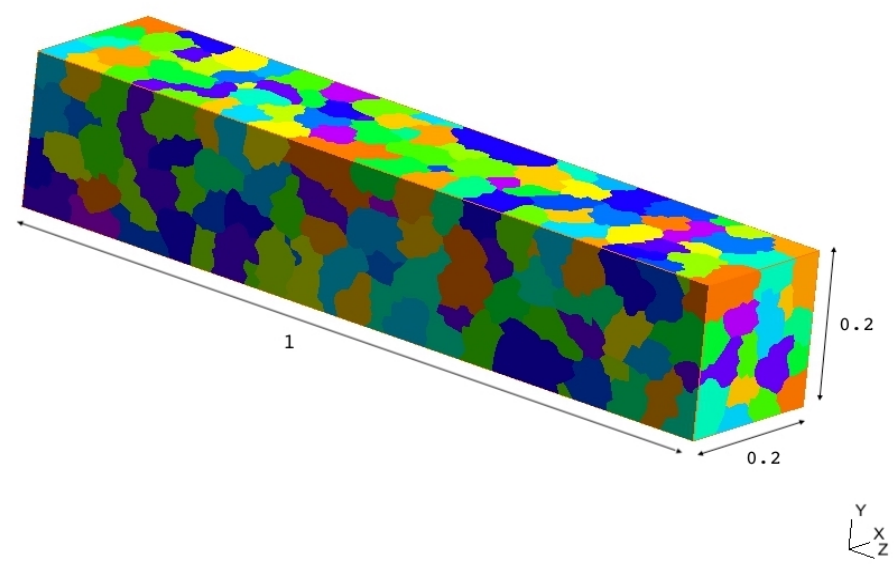

Figure 4.5: A typical three-dimensional finite element mesh partitioned into 320 subdomains.

experiments are performed with increasing number of random variables. In the subsequent sections, we will first discuss the experimental framework for the numerical simulations in Sec. 4.3.1. This is followed by the discussion on the characteristics of the solution process in Sec. 4.3.2. Next, in Sec. 4.3.3, we compare the scalability plots for the extended wirebasketbased coarse grid with the vertex-based coarse grid. Finally, in Sec. 4.3.4, we present the numerical and parallel scalability plots for the wirebasket-based BDDC/NNC solvers.

\subsubsection{Numerical Experimental Framework}

The PCGM Algorithm 1 presented in Sec. 3.2.1 is utilized to implement NCC/BDDC solver with the extended wirebasket-based preconditioner presented in Sec. 4.2. The solver is implemented by using Fortran programming language. Parallel processing is managed by using Message Passing Interface (MPI) communication routines [33]. Data structures and routines from PETSc [31] are used for subdomain-level (local) sparse matrix-vector storage and algebraic operations such as local sparse matrix-vector multiplications and local system solve. The finite element mesh is generated using GMSH [27, 202] and partitioned using METIS graph partitioner $[28,203]$. Stochastic system matrix and vector assembly is performed by employing 
element-level (deterministic) assembly routines from the FEniCS/dolfin [32]. The procedures from UQ Toolkit [29] are utilized to evaluate KLE and PCE basis functions and the corresponding multidimensional moments. For post processing and visualization, ParaView [34, 204] and Matlab [35] are employed. The simulations are performed on Canada's national HPC clusters managed by Compute Canada [205]. The nodes employed have either Intel Skylake cores running at 2.4 GHz from Niagara supercomputer [212] or Intel E5-2683 processors, running at 2.1 GHz from Cedar and Graham HPC systems [205].

\subsubsection{Characteristics of the Solution Process}

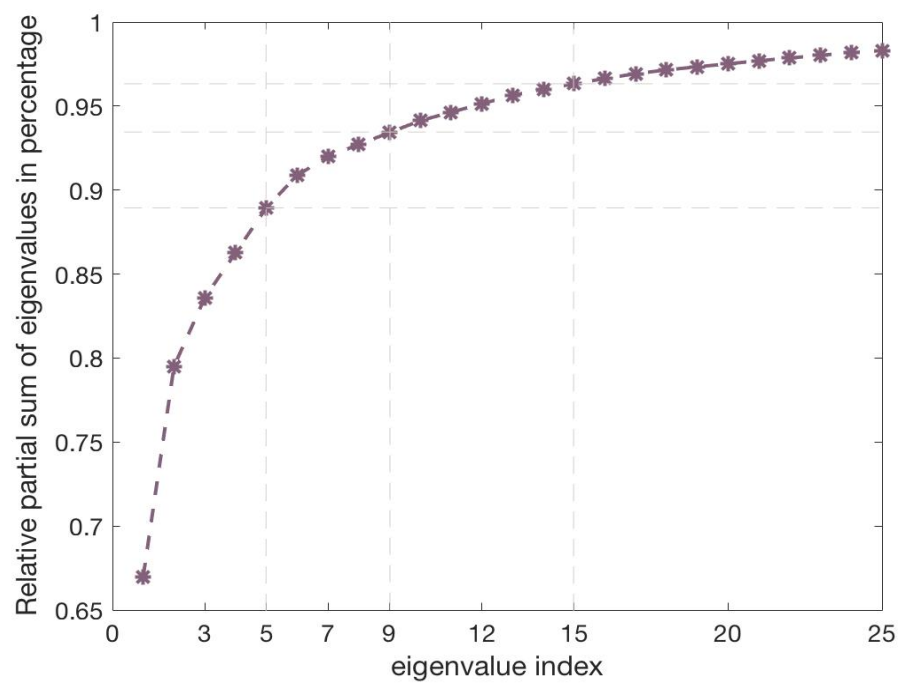

Figure 4.6: Relative partial sum of eigenvalues with respect to eigenvalue-index.

As a test case, consider five random variables $(L=5)$ and the second order expansion $\left(p_{\mathrm{A}}=2\right)$ for the PCE of the input stochastic process. Using $5 \mathrm{RVs}$ we can capture about $89 \%$ percent of the relative energy contribution for the given stochastic process. The relative energy contribution or the decay rate of the eigenvalues can be used as a guideline to determine the number of random variables required in the KLE to approximate the input stochastic process. For instance, using 9 RVs we can capture about $94 \%$ percent of the relative energy contribution and using 15 RVs we can capture about $97 \%$ of the relative energy contribution. See Fig. 4.6 
for the relative partial sum of eigenvalues for the respective eigenvalue index [1,24].

The PCE of the solution process with the five random variables $(L=5)$ and the third order expansion $P_{u}=3$ is used. For these parameters, we need 21 PCE terms for the characterization of the input stochastic process and 56 PCE terms for the representation of the output stochastic process [1]. The resulting coupled system of equations is solved for the PCE coefficients of the solution process $\mathscr{U}$ using in-house parallel BDDC/NNC solvers with wirebasket-based coarse grid discussed earlier in Sec. 4.2.

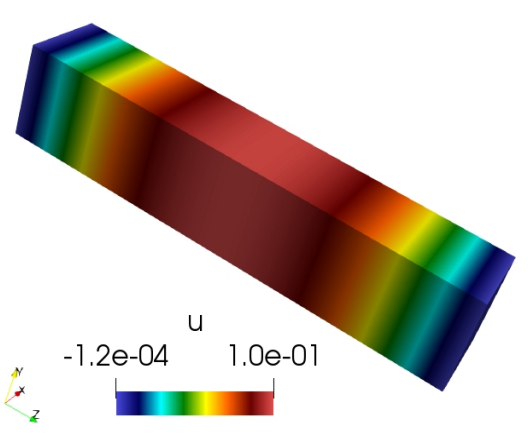

(a) mean $\mathcal{U}$

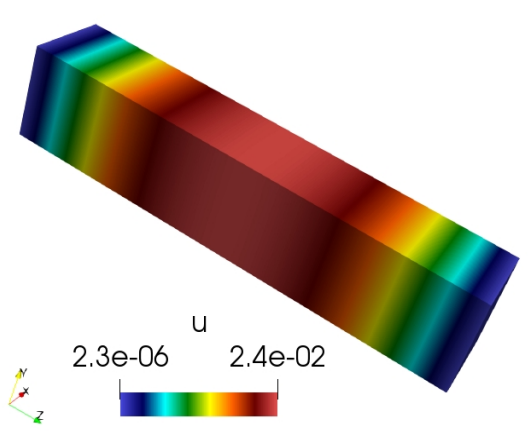

(b) standard deviation $\mathcal{U}$

Figure 4.7: Mean and standard deviation of the solution process $\mathcal{U}$.

The mean and standard deviation of the solution field $\mathcal{U}$ has the same trend, but, of course, their magnitudes are different. See Fig. 4.7 (a) and (b) respectively showing mean and standard deviation (SD) of the solution process. The maximum coefficient of variation in the centre of the domain is about $25 \%$. To get the further insights into the stochastic aspects of the solution process, the first few PCE coefficients are plotted in Figs. 4.8 to 4.10. One can see that, the PC coefficients exhibit oscillatory behavior and the magnitude of the chaos coefficients $u_{j}$ decreases with increasing PCE index $j$, i.e., the contribution of individual coefficients to the solution process decreases. Among these PCE coefficients, the first order coefficients contain Gaussian contributions and the higher order coefficients contain the non-Gaussian effects. As the order of expansion increases, the solution converges in terms of mean and standard deviation as shown in Fig. 4.7. 


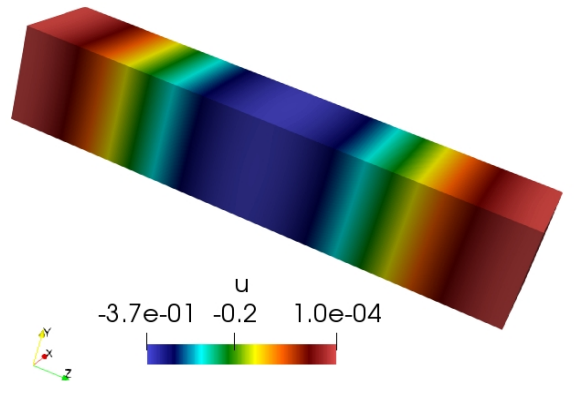

(a) PCE coefficient $u_{1}$

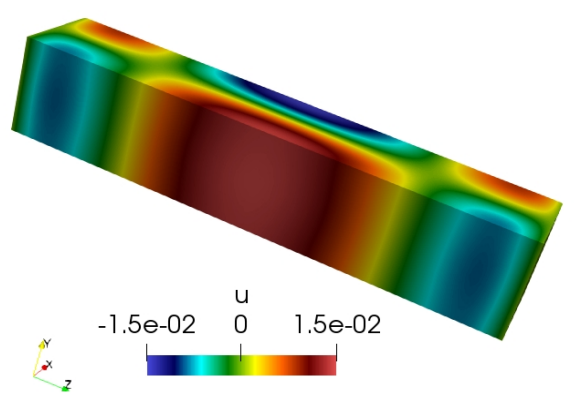

(c) PCE coefficient $u_{3}$

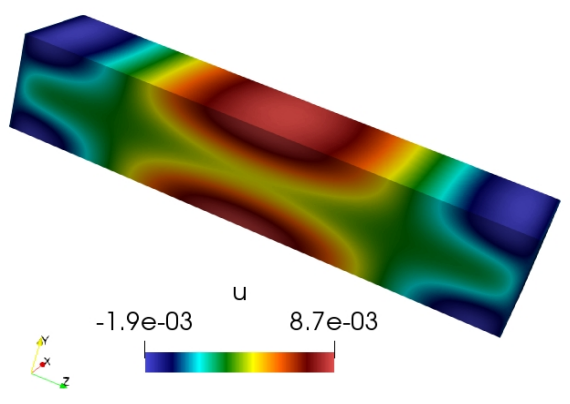

(e) PCE coefficient $u_{5}$

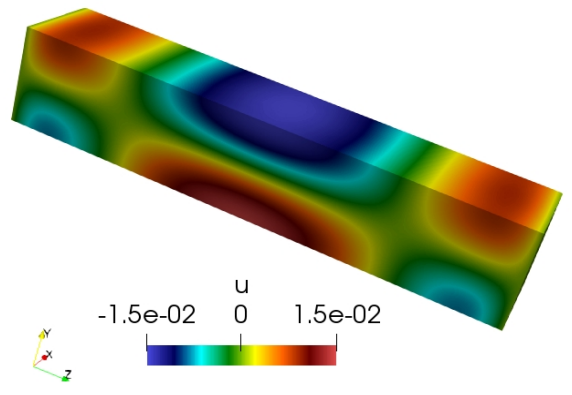

(b) PCE coefficient $u_{2}$

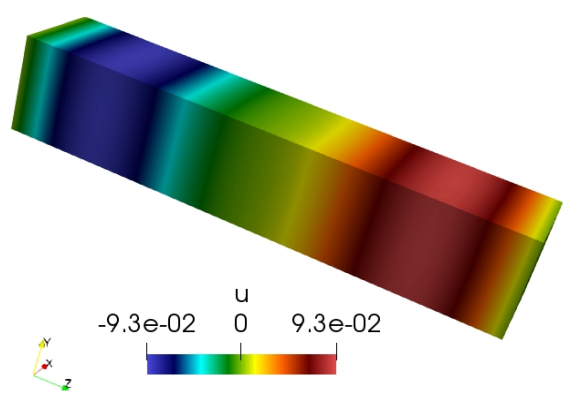

(d) PCE coefficient $u_{4}$

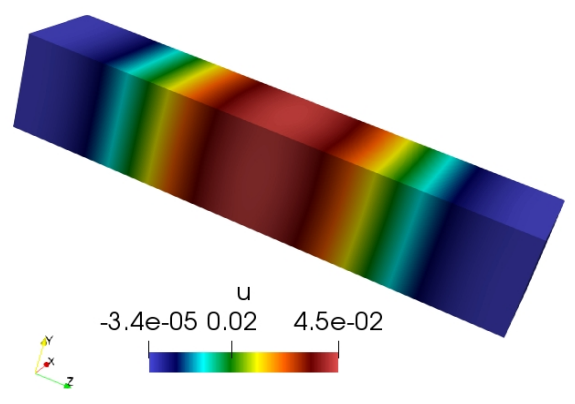

(f) PCE coefficient $u_{6}$

Figure 4.8: Selected PCE coefficients of the solution process $\mathscr{U}$. 


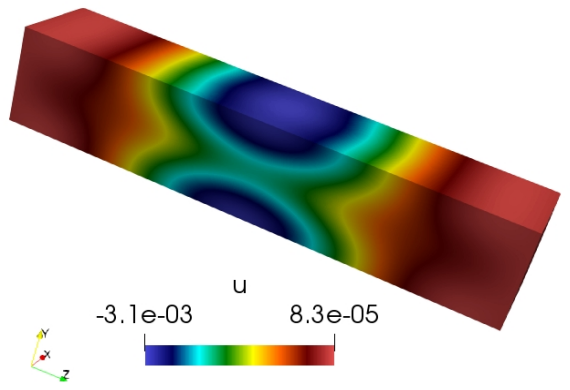

(a) PCE coefficient $u_{10}$

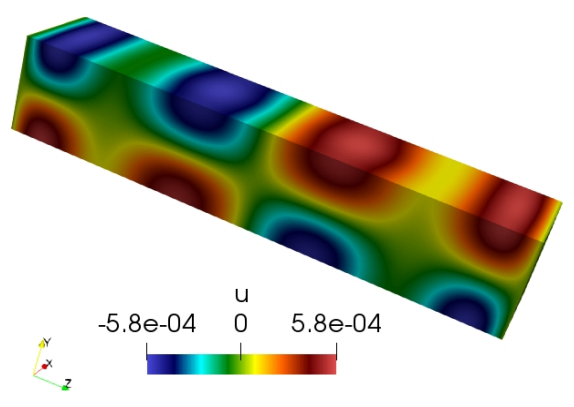

(c) PCE coefficient $u_{13}$

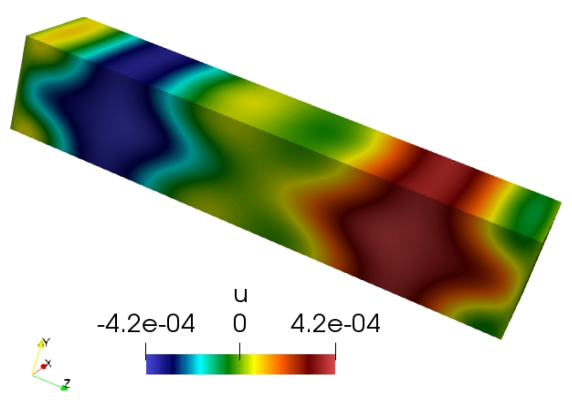

(e) PCE coefficient $u_{19}$

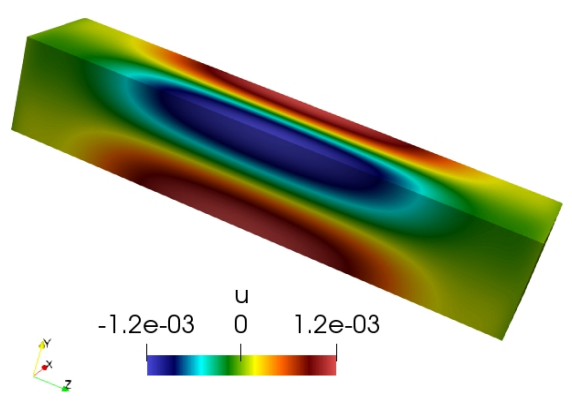

(b) PCE coefficient $u_{12}$

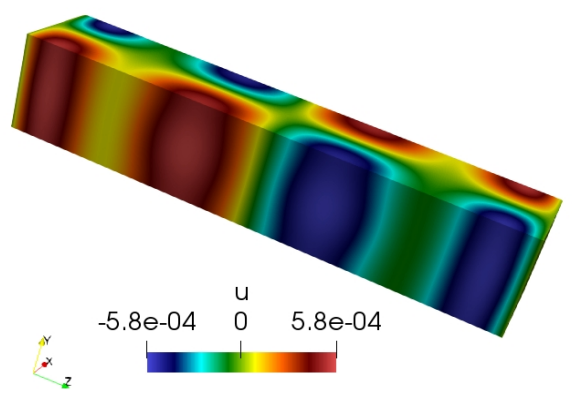

(d) PCE coefficient $u_{16}$

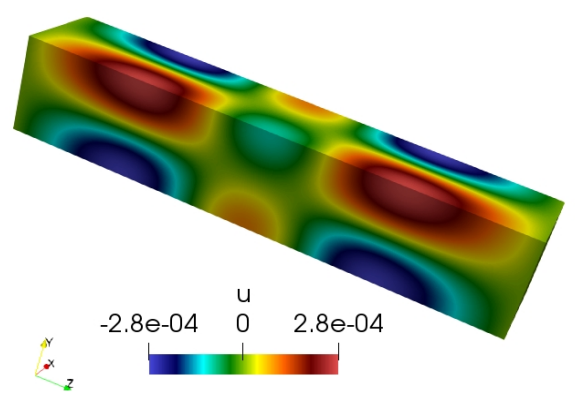

(f) PCE coefficient $u_{27}$

Figure 4.9: Selected PCE coefficients of the solution process $\mathscr{U}$. 


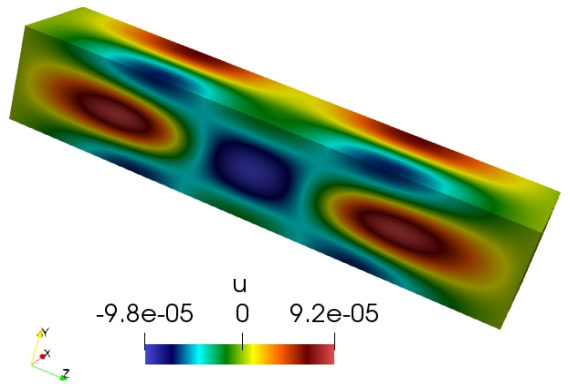

(a) PCE coefficient $u_{32}$

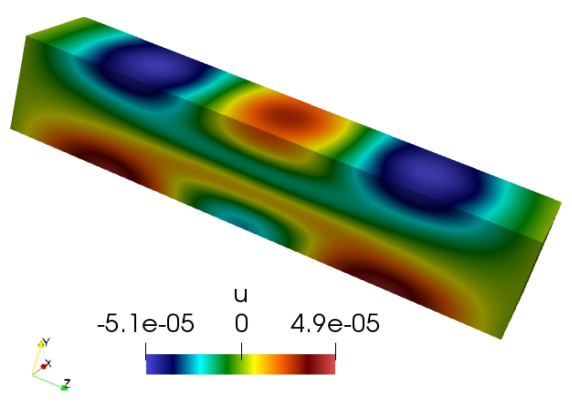

(c) PCE coefficient $u_{45}$

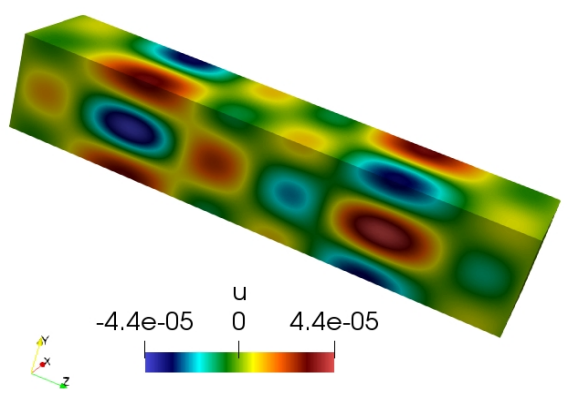

(e) PCE coefficient $u_{50}$

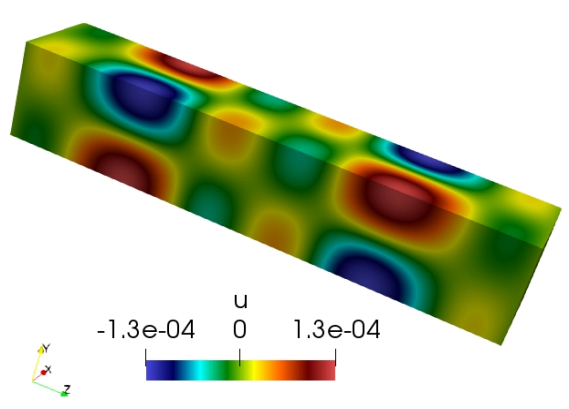

(b) PCE coefficient $u_{41}$

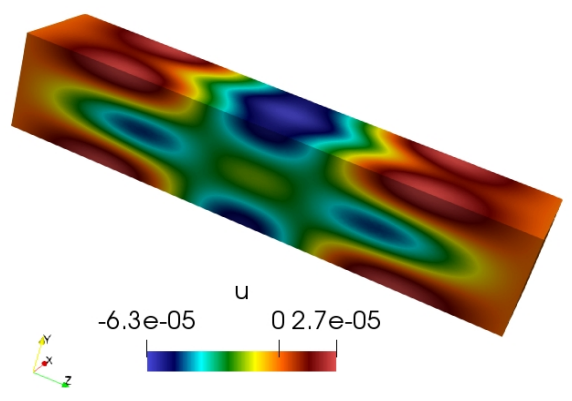

(d) PCE coefficient $u_{48}$

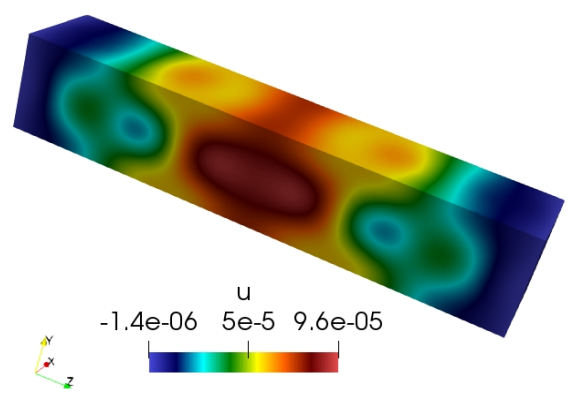

(f) PCE coefficient $u_{53}$

Figure 4.10: Selected PCE coefficients of the solution process $\mathcal{U}$. 


\subsubsection{Comparison of Extended Wirebasket with Vertex Coarse Grid}

The performance of extended wirebasket-based coarse grid preconditioner devised earlier is compared to that of the extended vertex-based coarse grid. In the current investigation, the primary focus is given to the performance comparison concerning numerical scalabilities of the solvers, i.e., the number of PCGM iterations required for the convergence with the given tolerance:

$$
\frac{\left\|\mathcal{U}_{\Gamma_{i+1}}-\mathcal{U}_{\Gamma_{i}}\right\|_{2}}{\left\|\mathcal{U}_{\Gamma_{i}}\right\|_{2}} \leq \text { tol }=10^{-5}
$$

with the subscript indicating the PCGM iteration number and $\|\cdot\|_{2}$ represents the $L_{2}$ norm. Additionally, other stopping criteria as detailed in [213] can be employed in this context.

The numerical scaling is essential to understanding the convergence behavior of the solver and it is considered crucial for the utility of the solver for the large-scale applications. The ideal numerical scaling would be the constant number of PCGM iterations with subdomains and/or problem size. Note that, the finite element mesh with the fixed resolution having 31598 nodes and 182681 (linear tetrahedral) elements is used for all simulations in this section. The maximum size of the linear system tackled in this section is about $\approx 7$ million.

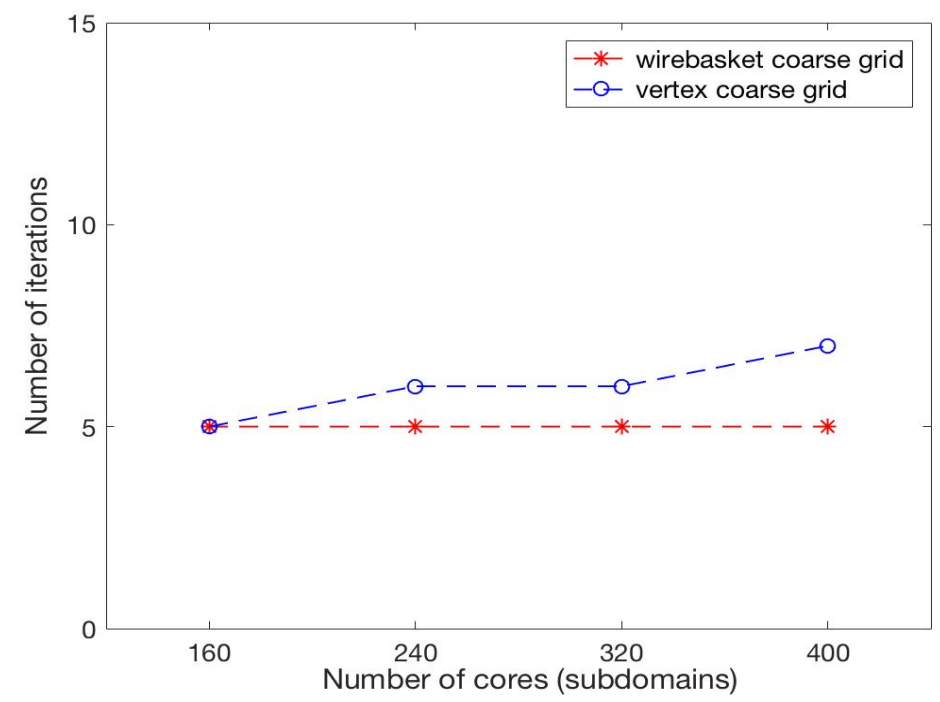

Figure 4.11: Iteration count versus number of subdomains for the fixed mesh resolution with fixed number of PCE terms. 
For a fixed mesh resolution with the fixed number of PCE terms $\left(P_{u}=56\right)$, if we increase the number of subdomains (accordingly number of cores), the number of PCGM iteration increases faster with the vertex-based coarse grid compared to that of the wirebasket-based coarse grid. Fig. 4.11 indicates that the BDDC/NNC solver with wirebasket-based coarse grid showed better performance in terms of number of PCGM iterations with respect to the number of subdomains compared to the vertex-based coarse grid.

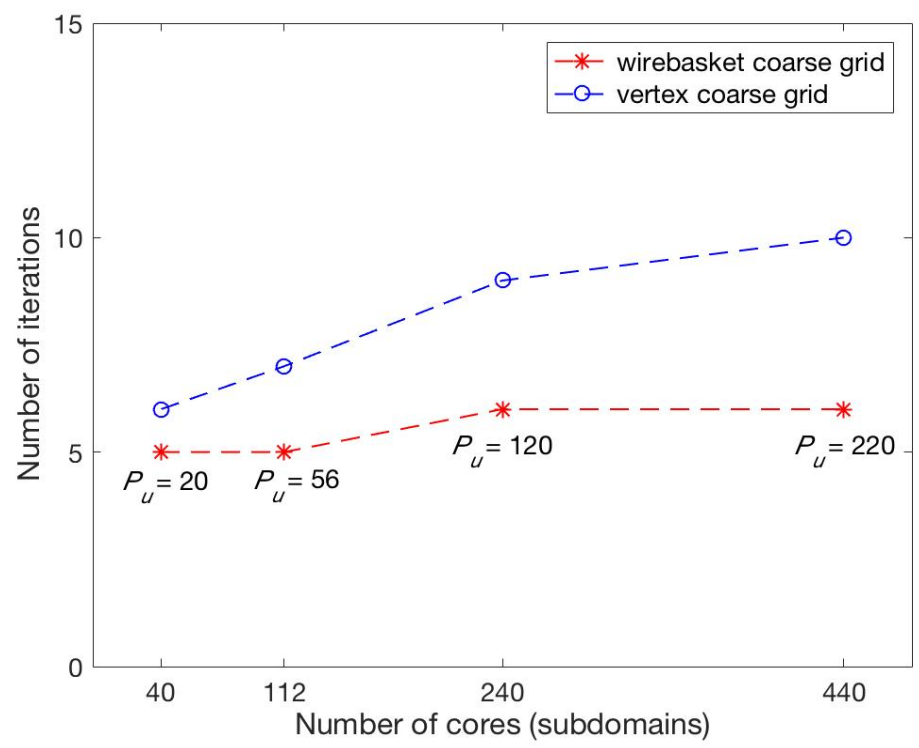

Figure 4.12: Iteration count versus number of subdomains for fixed problem size per subdomain with increasing number of PCEs (fixed mesh resolution).

For a fixed mesh resolution, the global problem size increases with the increasing number of PCE terms (i.e., $P_{u}=20,56,120$ and 220, corresponding to $L=3,5,6$ and 9, respectively). The ensuing larger linear system is then solved using more cores by keeping the problem size per core approximately the same $(\approx 15000)$. The PCGM iteration count for the BDDC/NNC with wirebasket-based coarse grid solver versus the number of subdomains (with the fixed problem size per subdomain) remains almost constant as shown in Fig. 4.12. On the other hand, for the same case, the number of iterations increases with the vertex-based coarse grid. Therefore, the BDDC/NNC solver with the wirebasket-based coarse grid scales better with respect to the number of PCE terms compared to the vertex-based coarse grid. For the same case as in 


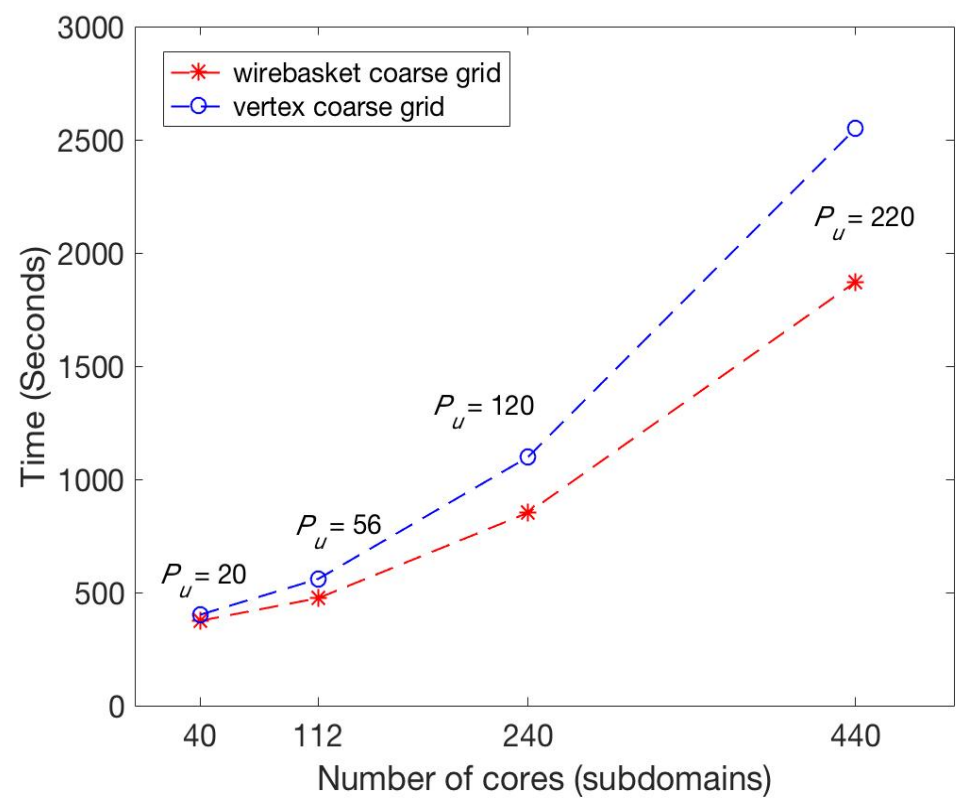

Figure 4.13: Execution time versus number of subdomains for fixed problem size per subdomain with increasing number of PCEs (fixed mesh resolution).

Fig. 4.12, the total execution time for the BDDC/NNC solver with wirebasket-based coarse grid and vertex-based coarse grid against the number of subdomains (with the fixed problem size per subdomain) are compared in Fig. 4.13. Total execution time for the BDDC/NNC solver with the vertex-based coarse grid increases faster compared to that of the wirebasket-based coarse grid. This is primarily due to more PCGM iteration required with the vortex-based coarse grid.

For a fixed spatial mesh resolution and the fixed number of subdomains $\left(n_{s}=384\right)$, an increase in the number of PCE terms by increasing the number of random variables (while maintaining the third-order expansion), results in almost constant PCGM iteration counts with the wirebasket-based based BDDC/NNC solver, as shown in Fig. 4.14. Conversely, for the same case, the number of PCGM iterations increases with the vertex-based coarse grid. Therefore, the BDDC/NNC solver with the extended wirebasket-based coarse grid is superior to vertexbased coarse grid concerning numerical scaling with respect to the number of random variables and the number of subdomains. 


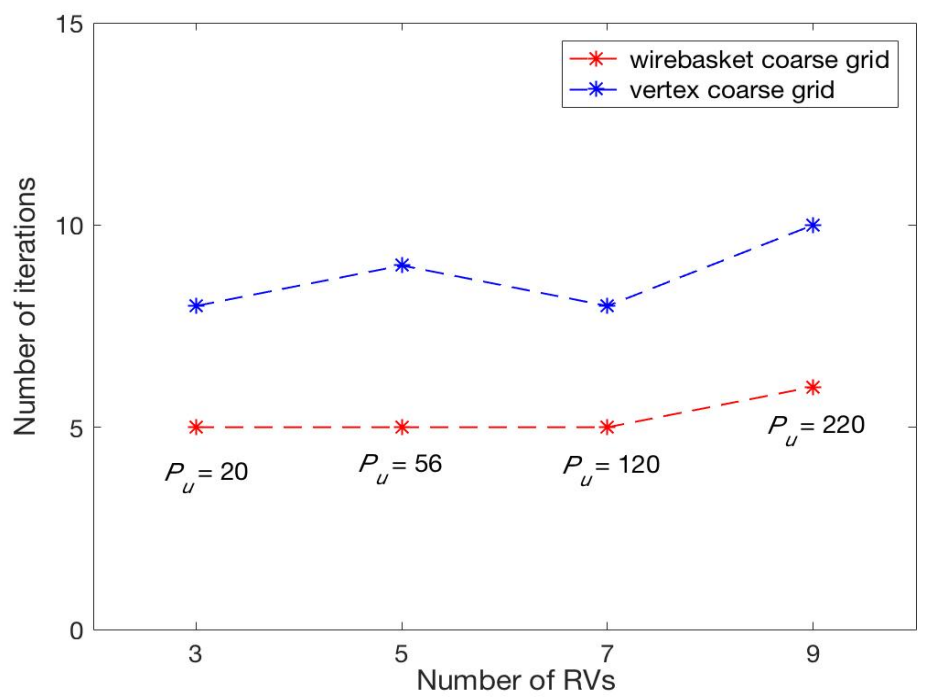

Figure 4.14: Iteration count versus number PCE terms for the fixed mesh resolution with fixed number of subdomains.

\subsubsection{Scalabilities Studies for Stochastic Simulations}

The main purpose of this study is to investigate the performance of the proposed BDDC/NNC solver with the wirebasket-based coarse grid for the problems with high dimensions in stochastic space. Therefore, for most of the simulations in this section, the finite element mesh with the fixed number of nodes 59741 and elements 350113 is used. For some cases, the mesh size is increased up to 159193 nodes and 947046 elements. To investigate the high-dimensional stochastic spaces, the performance plots for various cases by selecting the number of random variables ranging between 3 to 12 are presented. These cases are selected based on the relative contribution of the KLE eigenvalues (see Fig. 4.6).

The orders of expansion $p_{\mathrm{A}}=2$ and $p_{u}=3$ are used for the input and output PCE representations, respectively. Note that the orders of expansion, $p_{\mathrm{A}}$ and $p_{u}$ are kept constant for most of the experiments. For some cases $p_{u}$ is varied between 2 to 5 . With these KLE and PCE inputs, the number of PCE terms required to represent the solution process varies from 20 to 455 [1]. The maximum size of the system of linear equations simulated in this case is about 75 million, i.e., using finite element mesh with approximately 160 thousand node points and 455 PCE terms (12-RVs). 
In the subsequent sections, first, we study the numerical scalability of the solver concerning the number of PCGM iterations required for the convergence with the tolerance $t o l=10^{-5}$. Next, we examine the (strong and weak) parallel scalabilities in terms of time against the number of subdomains. Finally, we present the numerical and parallel scalability plots with respect to the stochastic parameters such as the number of random variables and the order of expansion.

\section{Numerical Scalability}

For a fixed problem size (59741 nodes and 350113 elements) with fixed number of PCE terms $\left(P_{u}=120\right.$ for $7 \mathrm{RVs}$ ), if we increase the number of subdomains, the PCGM iteration count almost remains constant. Fig. 4.15 indicates that the wirebasket-based BDDC/NNC-PCGM solver is numerically scalable with respect to the number of subdomains.

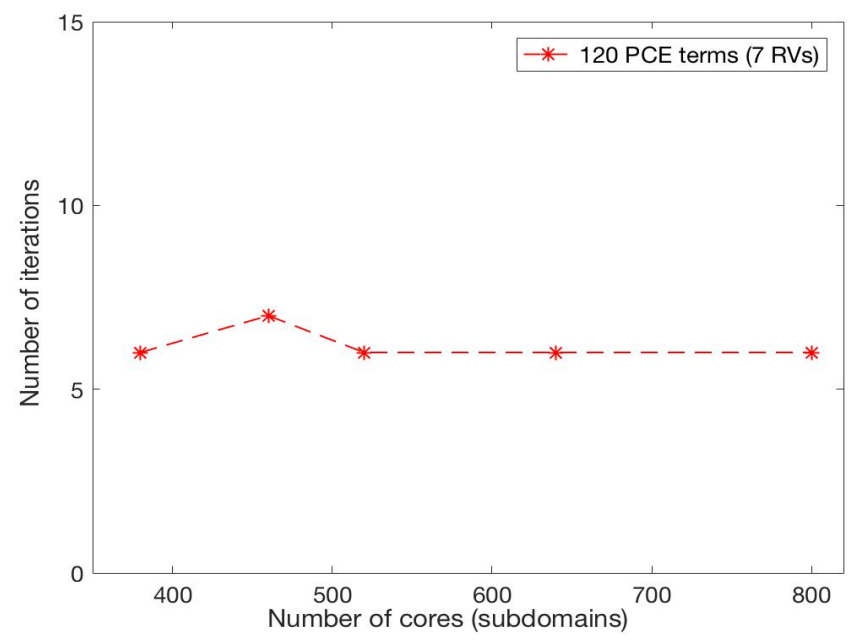

Figure 4.15: Iteration count versus number of subdomains for the fixed mesh resolution with fixed number of PCE terms.

Next, for the fixed number of PCE terms $\left(P_{u}=56\right.$ for $\left.5 \mathrm{RVs}\right)$, the global problem size is increased by increasing mesh resolution while keeping a fixed problem size per subdomain $(\approx 10500)$. As the overall problem size increases through the addition of new subdomains along with the coarse problem size, the PCGM iteration count slightly increases with increase in number of subdomains. Fig. 4.16 indicates that the BDDC/NNC-PCGM solver showed reasonable numerical scalability with respect to mesh resolution and fixed problem size per subdomain. 


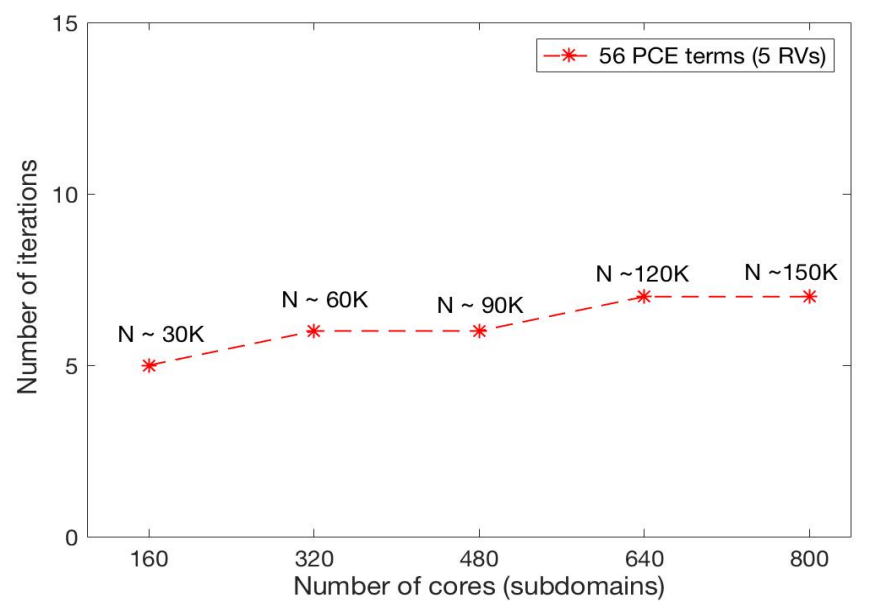

Figure 4.16: Iteration count versus number of subdomains for the fixed problem size per core with increasing mesh resolution (fixed number of PCE terms).

\section{Parallel Scalabilities}

The strong scalability of the BDDC/NNC-PCGM solver is plotted in Fig. 4.17. For a fixed spatial mesh resolution with fixed number of PCE terms $\left(P_{u}=120\right.$ for $\left.7 \mathrm{RVs}\right)$, an increase in the number of cores reduces the execution time as expected. Fig. 4.17 indicates excellent strong scaling of the BDDC/NNC solver with the wirebasket-based coarse grid. Similar behavior is observed in the cases with three and five random variables (although not shown here).

The weak scaling of the wirebasket-based BDDC/NNC-PCGM solver is plotted in Fig. 4.18. For the fixed number of PCE terms ( $P_{u}=56$ for $\left.5 \mathrm{RVs}\right)$, the global problem size is increased by increasing mesh resolution while keeping a fixed problem size per subdomain $(\approx 10500)$. The growth in the total execution time implies that the probabilistic BDDC/NNC solver shows poor weak scaling. Similar behavior is also observed in the cases of probabilistic BDDC/NNC solver for the two-dimensional stochastic diffusion equations discussed in Chapter 2. The suboptimal weak scaling of the solver is mainly because of the increased parallel overhead with the number of cores caused by the MPI collective communication [19,24]. Also, the size of the global coarse problem increases with increasing number of subdomains; this is more so in the case of the wirebasket-based coarse grid, demanding more computational efforts. 


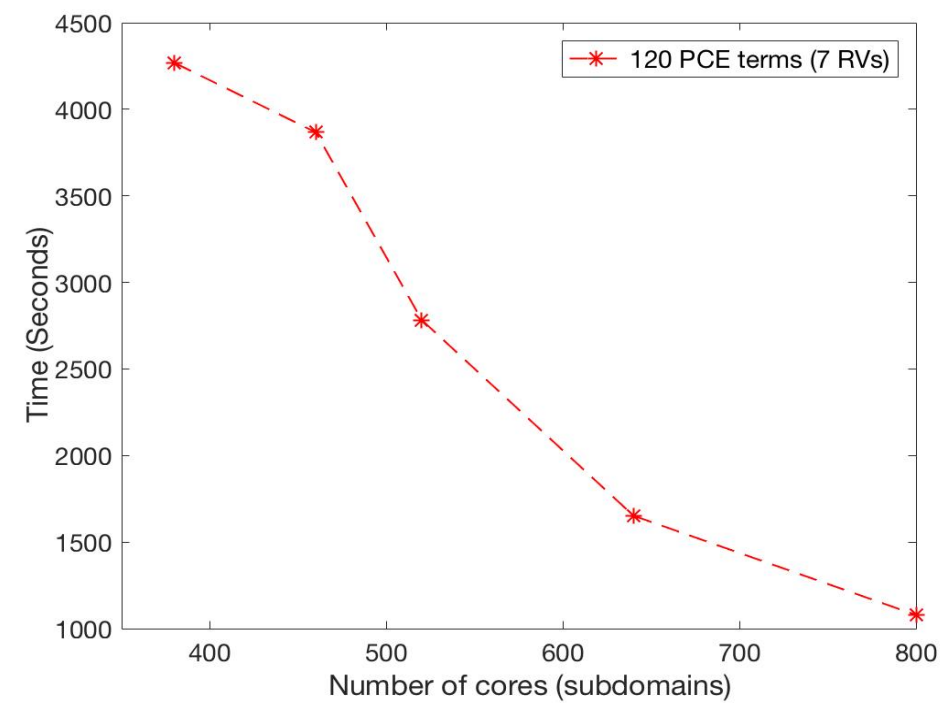

Figure 4.17: Execution time versus number of subdomains with the fixed mesh resolution and the number of PCE terms.

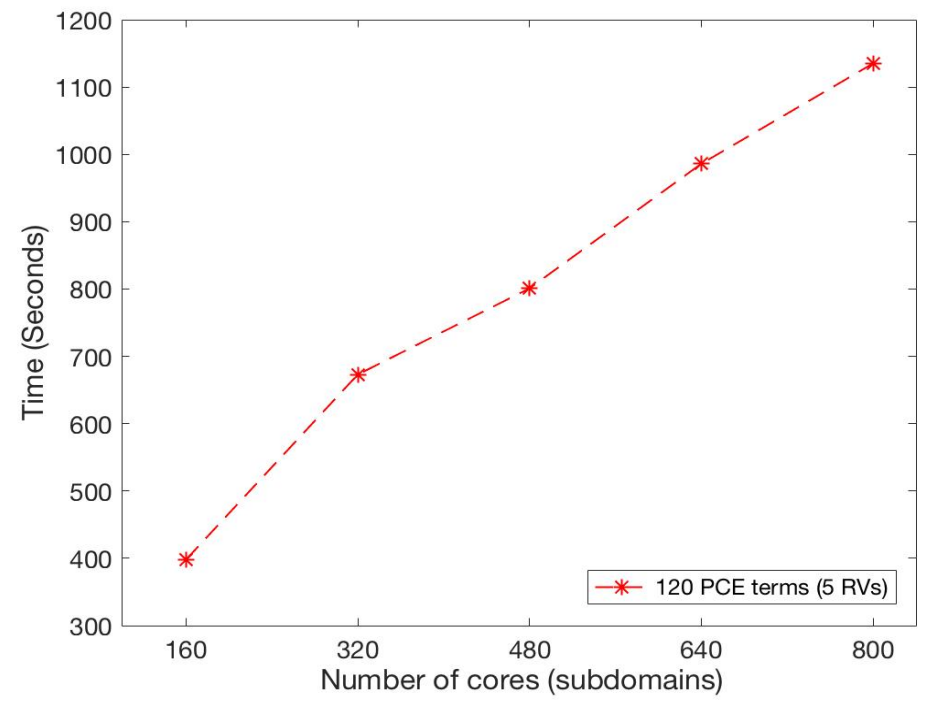

Figure 4.18: Execution time versus number of subdomains for the fixed problem size per core with increasing mesh resolution (fixed number of PCE terms). 


\section{Scalability with respect to Stochastic Parameters}

To investigate the effects of stochastic aspects of the problem on the scalability of the solver, the numerical and parallel scaling with respect to the number PCE terms and the order of expansions are presented in this section. The four points in Figs. 4.19 to 4.21 corresponding to the number of PCE terms 20, 56, 120 and 220 are related to the number of random variables 3, 5, 7 and 9, respectively. The finite element mesh resolution corresponds to 59741 nodes and 350113 elements.

For a fixed mesh resolution and fixed number of subdomains $\left(n_{s}=640\right)$, an increase in the number of PCE terms by increasing the number of random variables (while maintaining the third-order expansion) results in almost constant PCGM iteration counts as shown in Fig. 4.19. This result demonstrates that the wirebasket-based BDDC/NNC-PCGM solver is numerically scalable with respect to the number of random variables. Although the total problem size increases with the number of PCE terms, one can solve the larger system with almost the same number of PCGM iterations.

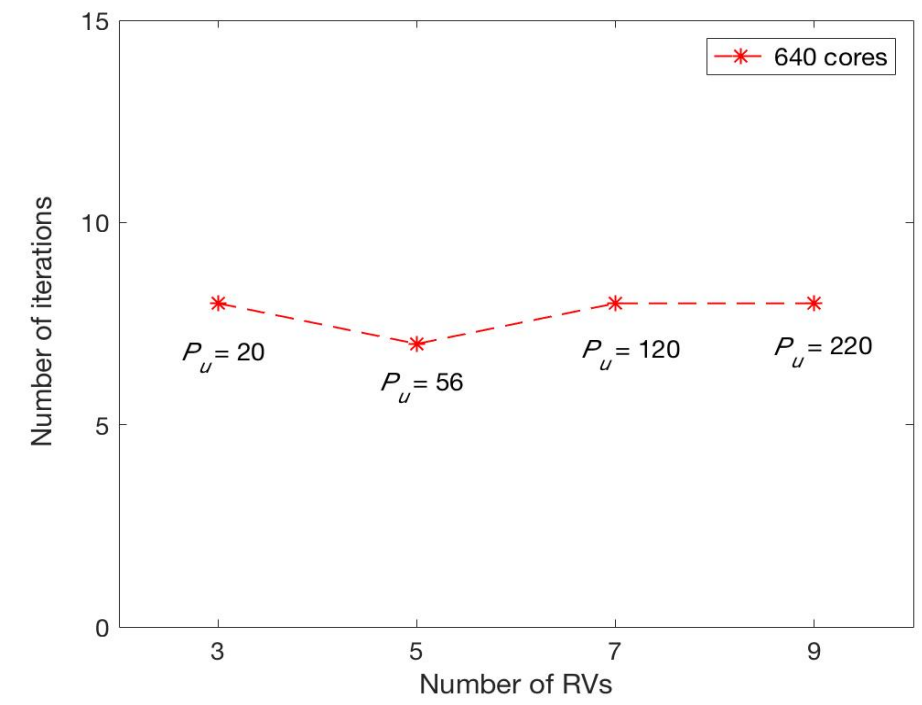

Figure 4.19: Iteration count versus number PCE terms for the fixed mesh resolution with fixed number of subdomains.

For a fixed mesh resolution with the increasing number of PCEs, the global problem size increases. The ensuing larger linear system is then solved using more cores by keeping the prob- 


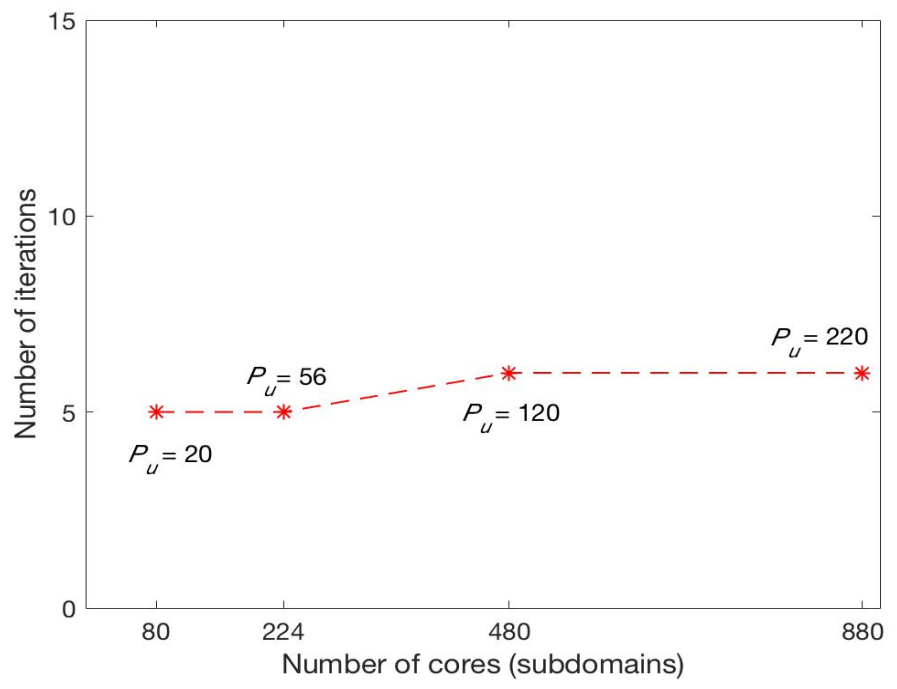

Figure 4.20: Iteration count versus number of subdomains for the fixed problem size per core with increasing number of PCE terms (fixed mesh resolution).

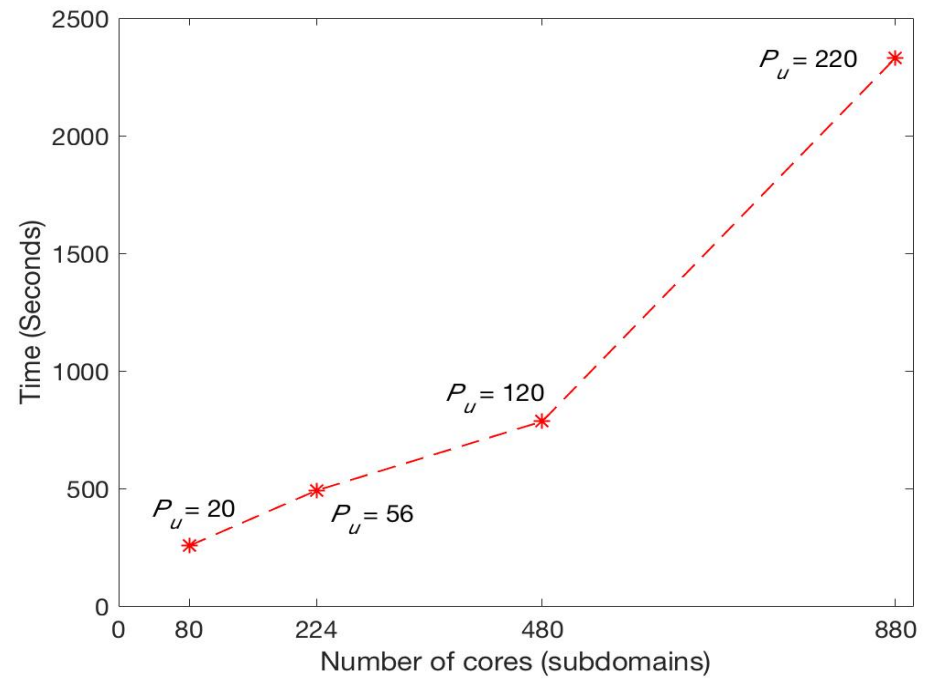

Figure 4.21: Execution time versus number of subdomains for fixed problem size per subdomain with increasing number of PCEs (fixed mesh resolution). 


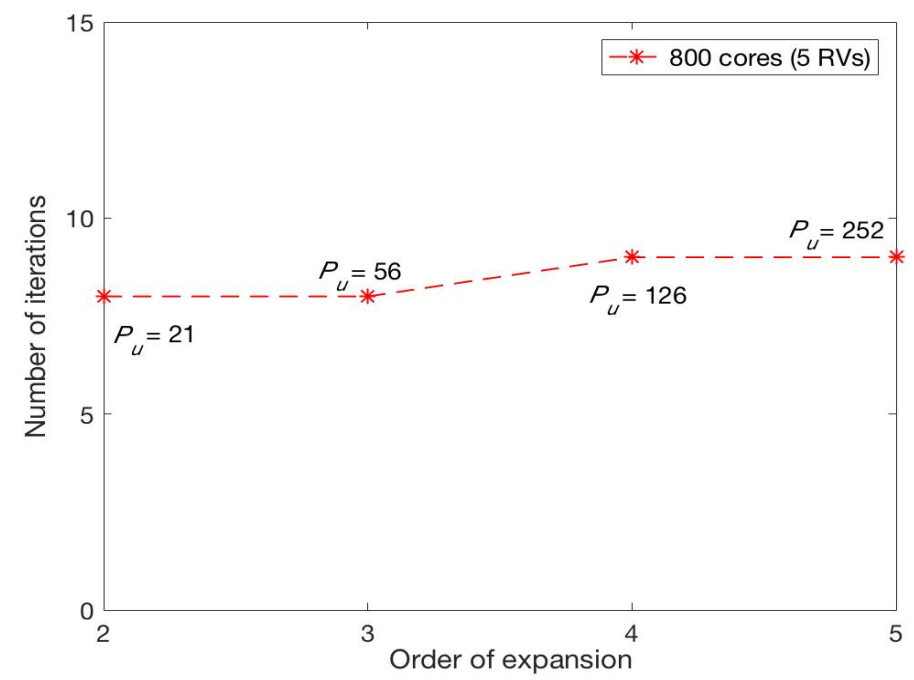

Figure 4.22: Iteration count versus order of expansion for the fixed mesh resolution with fixed number of subdomains (fixed number of RVs).

lem size per core approximately the same $(\approx 15000)$. The iteration count for the wirebasketbased DDC/NNC-PCGM solver versus the number of subdomains (with the fixed problem size per subdomain) remains almost constant as shown in Fig. 4.20. Hence the wirebasket-based BDDC/NNC-PCGM solver is numerically scalable with respect to the number of PCE terms.

The total execution time for the solver against the number of subdomains, with fixed problem size per subdomain for third order expansion and 3, 5, 7 and 9 RVs) is illustrated in Fig. 4.21. The execution time increases with respect to the PCE terms and the number of subdomains although the problem size per core is nearly the same. This is primarily due to increased MPI collective communication overhead. Additionally, as pointed out earlier in Chapter 3, the increasing number of PCE terms leads to an extended system of coupled equations and the new system inherits a more complex coupling structure. Therefore, the new system demands more computational effort.

For a fixed mesh resolution and fixed number of subdomains $\left(n_{s}=800\right)$, an increase in the number of PCE terms by increasing the order of expansion $p_{u}$ (while maintaining the fixed number of random variables $L=5$ ) results in almost constant PCGM iteration counts as shown in Fig. 4.22. This result suggests that the BDDC/NNC-PCGM solver is numerically scalable 
with respect to the order of expansion. Note that, the coupling structures resulting from increasing number of PCE terms by increasing the number of random variables are more complex and computationally demanding than those resulting from increasing order of expansion. For instance, see Fig. 2.3 and 2.4 showing the effects of increasing number of RVs and the order of expansion on to the number of non-zero elements and coupling structures of the resulting system matrices.

\subsection{Three-Dimensional Linear Elasticity Problem}

Application of deterministic finite element method to the equations of linear elasticity results in an extremely large, coupled system of linear equations for high resolution meshes. This linear system is often much more computationally expensive than those resulting from finite element discretization of scalar-valued elliptic PDEs, such as diffusion equation [128, 139]. Therefore solution of such system demands extensive computational efforts [128, 139, 208]. Moreover, extending the problem in stochastic space by using polynomial chaos expansion based intrusive SSFEM adds an additional coupling in the resulting system of equations and further complicates the system [24]. This coupling among the polynomial chaos coefficients of the vector-valued solution process results in complicated block structure of the stochastic system matrix. Therefore, this can further influence the condition number of the global Schur complement system resulting from non-overlapping domain decomposition method. To tackle such system, a simple vertex-based coarse grid is inefficient [24], as we need a much stronger mechanism such as a wirebasket coarse grid to communicate information globally [24,208]. In this section, we employ the two-level BDDC/NNC preconditioner using extended wirebasketbased coarse grid discussed in Sec. 4.2, to simulate the stochastic system of coupled equations in linear elasticity.

To simplify the application of DD-based intrusive SSFEM to a coupled stochastic PDE system, we have presented a detailed formulation for the extended Schur complement system for 
coupled stochastic PDE system in Sec. 4.1. The efforts are made to explain the two-levels of couplings arising in the setting of DD-based intrusive SSFEM due to PC expansion of vectorvalued solution process. In Sec. 4.4.1, an experimental framework for the numerical simulations of a clamped beam is discussed. This is followed by the discussion on the characteristics of the stochastic solution process in Sec. 4.4.2. The Sec. 4.4.3 is dedicated to comparing the numerical scalability of BDDC/NNC solver with the extended wirebasket-based coarse grid against the vertex-based coarse grid. Finally in Sec. 4.4.4, the numerical and parallel scalability results of wirebasket-based BDDC/NNC solver for a clamped beam deformed due to self-weight are presented.

In this section, we exploit extended wirebasket-based BDDC/NNC preconditioners to solve the Schur complement system for a coupled stochastic PDE system exemplified through linear elasticity problems. For an exposition of the methodology, we consider the equations of linear elasticity to model the vector-valued stochastic displacement field $\mathcal{U}(\boldsymbol{x}, \theta)$ of a three-dimension body defined over the volume $\mathcal{D}(x, y, z)$. The resulting equations can be written as $[26,139$, 208],

$$
\begin{aligned}
-\nabla \cdot \sigma(\mathcal{U}(\boldsymbol{x}, \theta)) & =F(\boldsymbol{x}) \quad \text { in } \quad \mathcal{D}, \\
\sigma(\mathcal{U}(\boldsymbol{x}, \theta)) \cdot \hat{\mathbf{n}} & =b_{T} \quad \text { on } \quad \Gamma_{1}=\delta \mathcal{D} \backslash \Gamma_{0}, \\
\mathcal{U}(\boldsymbol{x}, \theta) & =0 \quad \text { on } \quad \Gamma_{0},
\end{aligned}
$$

where $\sigma$ is the stress tensor, $F$ is the body force vector per unit volume, $\hat{\mathbf{n}}$ is outward unit normal on boundary $\Gamma$ and $b_{T}$ is the traction on boundary $\Gamma_{1}$. For homogeneous and isotropic linear elasticity the stress tensor $\sigma$ can be written as [32,139]

$$
\sigma(\mathcal{U}(\boldsymbol{x}, \theta))=\lambda(\nabla \cdot \mathcal{U}(\boldsymbol{x}, \theta)) I+2 \mu \epsilon
$$

where $I$ is the identity matrix, the $\mu$ and $\lambda$ are Lamé constants and $\epsilon$ is the symmetric strain 
tensor denote as

$$
\epsilon=\frac{1}{2}\left(\nabla \mathcal{U}(\boldsymbol{x}, \theta)+(\mathcal{U}(\boldsymbol{x}, \theta))^{\mathrm{T}}\right)
$$

To construct the variation form we take inner product of Eq. (4.40) with test function $\mathcal{V}$ and integrate over the domain $\mathcal{D}$,

$$
\int_{\mathcal{D}}(-\nabla \cdot \sigma(\mathcal{U})) \cdot \mathcal{V} d x=\int_{\mathcal{D}} F \cdot \mathcal{V} d x
$$

Integrating by parts, Eq. (4.45) becomes (note $\boldsymbol{x}$ and $\theta$ of $\mathcal{U}$ are dropped),

$$
\int_{\mathcal{D}} \sigma(\mathcal{U}): \nabla \mathcal{V} d x=\int_{\mathcal{D}} F \cdot \mathcal{V} d x+\int_{\Gamma_{1}} b_{T} \cdot \mathcal{V} d s
$$

where the operator : is the inner product between tensors. $b_{t}$ is prescribed on a part $\Gamma_{1}$ of the boundary. Therefore, the integral on the remaining part of the boundary $\Gamma_{0}$ vanishes due to a Dirichlet boundary condition. The Eq. (4.46) can be re-written more concisely using bilinear and linear forms as,

$$
a(\mathcal{U}, \mathcal{V})=\mathcal{L}(\mathcal{V})
$$

Lamé constants arising in the stress-strain relationship for the material can be written as the functions of Poisson ratio $\nu$ and Young's modulus $E$ as follows [32,139],

$$
\lambda=\frac{E \nu}{(1+\nu)(1-2 \nu)}, \quad \mu=\frac{E}{2(1+\nu)} .
$$

For simplicity, we consider Poisson's ratio as a spatially invariant parameter and model Young's modulus as the lognormal stochastic process representing spatially varying uncertainty,

$$
E(\boldsymbol{x}, \theta)=E_{0}(\boldsymbol{x}) \exp (g(\boldsymbol{x}, \theta))
$$


where $E_{0}(\boldsymbol{x})$ represents the mean and $g(\boldsymbol{x}, \theta)$ denotes the underlying Gaussian process with the variance $\sigma^{2}$ and the exponential covariance function $C$ defined in Eq. (4.50) [1,24].

$$
C\left(x_{1}, y_{1}, z_{1} ; x_{2}, y_{2}, z_{2}\right)=\sigma^{2} e^{-\left|x_{2}-x_{1}\right| / b_{x}-\left|y_{2}-y_{1}\right| / b_{y}-\left|z_{2}-z_{1}\right| / b_{z}}
$$

where $b_{x}, b_{y}$ and $b_{z}$ are the correlation lengths along $x, y$ and $z$ directions respectively. While the assumption of the spatial invariance on the Poisson's ratio simplifies the analysis, the spatial variability of both Poisson's ratio and Young's modulus can be handled concurrently as the proposed framework is general [1,214,215].

\subsubsection{Numerical Experimental Framework}

The PCGM Algorithm 1 presented in Chapter 3, is implemented by the author for the extended wirebasket-based BDDC/NNC solver presented in Sec. 4.2, using Fortran programming language. Parallel processing is managed by using MPI communication routines [33]. Data structures and routines from PETSc [31] are used for subdomain-level (local) sparse matrix-vector storage and algebraic operations such as local sparse matrix-vector multiplication and local system solve. An unstructured finite element mesh is generated using GMSH [27] and partitioned using METIS graph partitioner [28]. Stochastic system matrix and vector assemblies are performed by employing element-level (deterministic) assembly routines from the FEniCS/dolfin [32]. The procedures from UQ Toolkit [29] are utilized to evaluate KLE and PCE basis functions and the corresponding multidimensional moments. For post processing and visualization, ParaView [34, 204] and Matlab [35] are employed.

The simulations are performed on Canada's national HPC clusters managed by Compute Canada [205]. The nodes employed have either Intel Skylake cores running at $2.4 \mathrm{GHz}$ from Niagara supercomputer [212] or Intel E5-2683 processors, running at $2.1 \mathrm{GHz}$ from Cedar and Graham HPC systems [205]. Similar to the earlier cases, the primary focus is given to investigate the performance of the solver to handle the high-dimensional coupled stochastic 
systems. In all the simulations we use (four node) linear tetrahedral elements. Such low order linear finite elements are shown to be sufficient for elliptic problem with compressible elastic materials [216,217]. In the subsequent sections, we will first discuss the characteristics of the solution process followed by the results for numerical and parallel scalabilities of the proposed solver by varying the number of random variables.

\subsubsection{Characteristics of the Solution Process}

As a test case, consider a clamped beam of length $L_{b}$ with a square cross section of width $W$, deformed under self-weight in three dimensions. A typical finite element mesh for such beam with 31598 nodes and 182681 linear tetrahedral elements, partitioned into 320 subdomains is shown in Fig. 4.23.

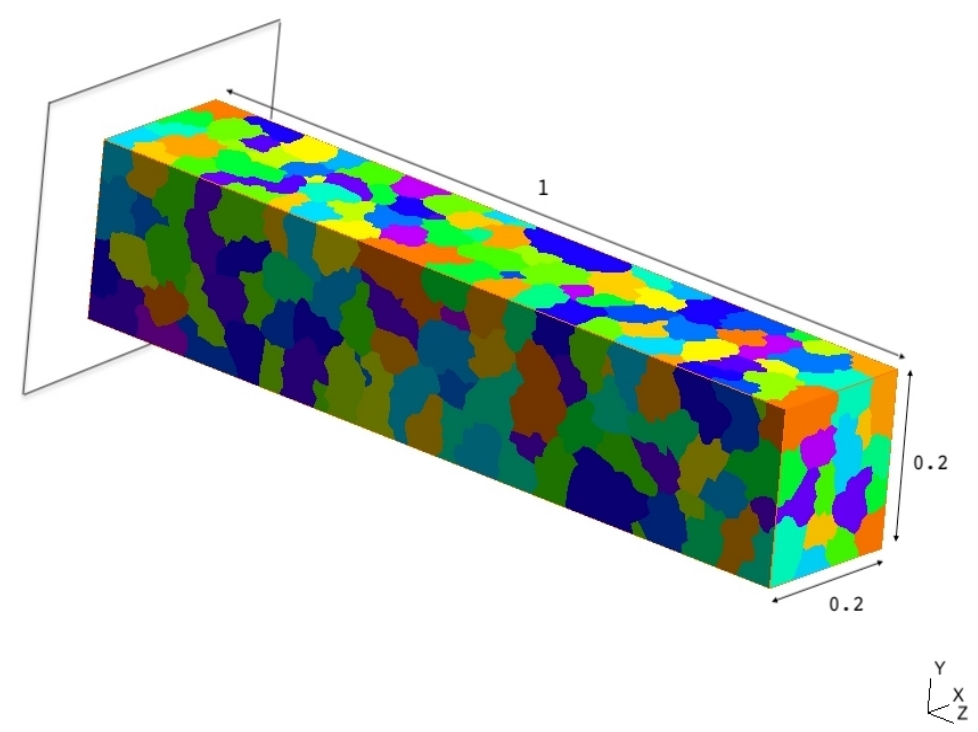

Figure 4.23: A typical three-dimensional finite element mesh for a clamped beam partitioned into 320 subdomains.

For the numerical experiments we have used the nondimensionalized and scaled system parameters suggested in FEniCS tutorial [32]. The parameters are made dimensionless by using beam length and $\overline{\mathcal{U}}=\mathcal{U} / \mathcal{U}_{m}$. The scaling is achieved by choosing $\mathcal{U}_{m}$ equal to the maximum 
deflection of a clamped beam (refer to FEniCS tutorial [32] for further details). The advantage of using scaled problem is to reduce the need for setting the physical parameters and also, the obtained dimensionless numbers can be used to understand the competition of parameters and physical effects. Furthermore, the dimensionless parameters simplify the computational model and make it ideal for numerical experiments. The following values are used for numerical simulations (as suggested in FEniCS tutorial [32]); Lamé constants are fixed to $\mu=1$ and $\lambda=1.25$. The density of the beam is set to $\rho=1$ and the acceleration due to gravity is set to $g=0.4\left(W / L_{b}\right)^{2}$. The beam is deformed under its weight with $F=(0,-\rho g, 0)$, where $F$ is the body force per unit volume. At the clamped end of the beam, i.e., on $\Gamma_{0}, \mathcal{U}=(0,0,0)$. The rest of the boundary $\Gamma_{1}$ is traction free, i.e., $b_{T}=0$.

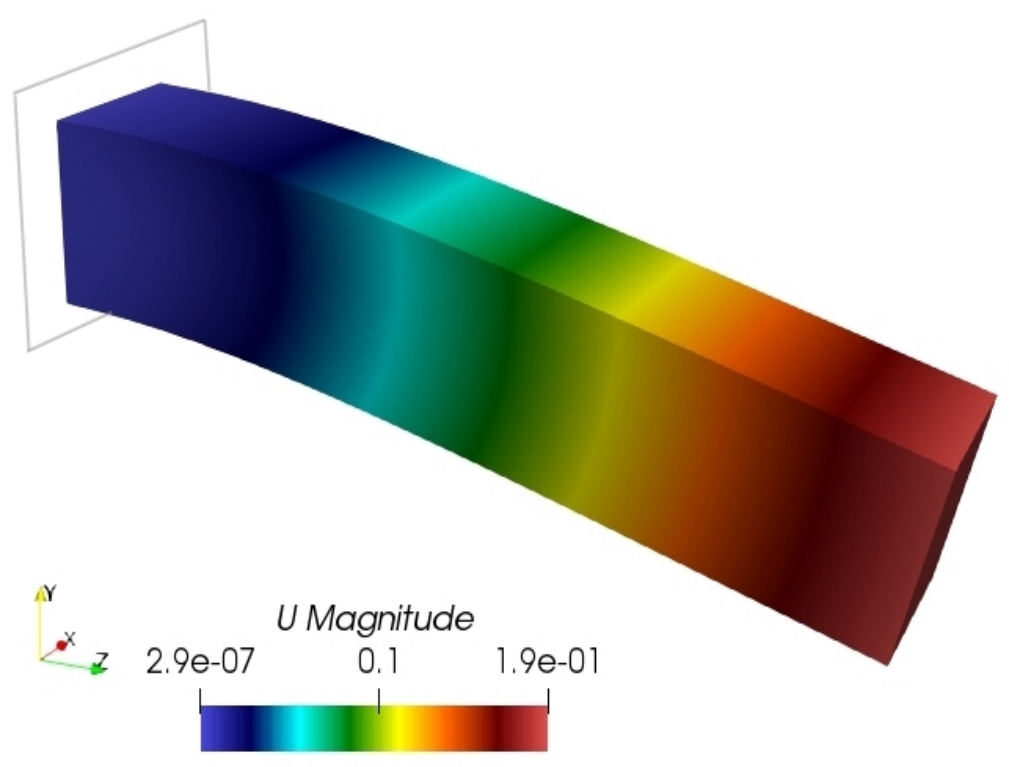

Figure 4.24: Mean magnitude of the beam deflection subjected to self-weight.

For simplicity, we consider Poisson's ratio as spatially invariant with $\nu=0.2778$ and model Young's modulus $E$ as a non-Gaussian stochastic process representing spatially varying uncertainty as shown in Eq. (4.49). The mean value for dimensionless Young's modulus $E_{0}=2.556$ is calculated from Eq. (4.48) using $\mu=1$ and $\lambda=1.25$. Young's modulus is characterized as a lognormal stochastic process with underlying Gaussian process having $\mu=0, \sigma=0.3$ and 
exponential covariance kernel with the correlation lengths $b_{x}=b_{y}=b_{z}=1$. The PCE of the input stochastic process is represented using three random variables $(L=3)$ and the second order expansion $\left(p_{a}=2\right)$. The PCE of the solution process with $L=3$ and $p_{u}=3$ is used. For the selected $L$ and $p_{\alpha}$, we need 10 PCE terms for the input and 20 PCE terms for the output [24].

The mean value of the magnitude of the deflected beam is shown in Fig. 4.24. As expected, the maximum deflection occurs at the free end of the beam. The maximum coefficient of variation in the magnitude of the displacement field is about $26 \%$ which emphasizes the effect of input uncertainty. The mean and standard deviation (SD) of the magnitude of $\mathcal{U}$ and its spatial components $\mathcal{U}_{x}, \mathcal{U}_{y}$ and $\mathcal{U}_{z}$ are shown in Fig. 4.25. The magnitude of the mean and SD have the same trend but the components of the displacement vector exhibit more complicated features. The mean of the magnitude of deflection at the mid-span and the free end of the beam approximately matches with the analytical solution obtained at the mean values of the system parameters.

To get further insights into the stochastic aspects of the solution process, the first few PCE coefficients showing the magnitude and the respective displacement components of the solution process are plotted in Figs. 4.26 to 4.29. Similar to the earlier observations, the contribution of the magnitude of chaos coefficients $u_{j}$ (and the respective displacement components) to the solution process decreases with increasing PCE index $j$. For example, the magnitude of PCE coefficient for $j=1$ is in the order of $10^{-2}$ as shown in Fig. 4.26 (a). However, for instance, the magnitudes of PCE coefficiet for $j=6, j=10$ and $j=19$ are in the order of $10^{-4}, 10^{-5}$ and $10^{-6}$ as shown in Fig. 4.27 (b), Fig. 4.28 (a) and Fig. 4.29 (b), respectively. Similar effects are observed in the respective displacement components as shown in Figs. 4.26 to 4.29. Among these coefficients, the first order coefficients contain Gaussian contributions, for example, as shown in Fig. 4.26. The higher order coefficients, as shown in Figs. 4.27 to 4.29, contain the non-Gaussian effects. As the number of PCE terms increases, the solution converges in terms of the mean and standard deviation as shown in Fig. 4.25. 


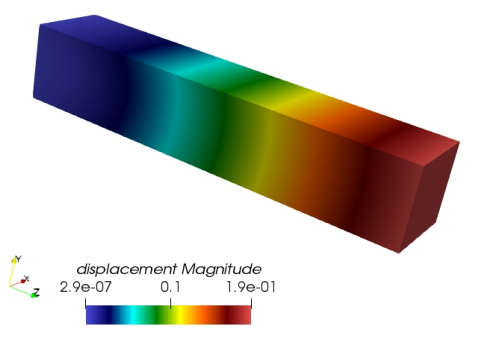

(a) Mean: magnitude

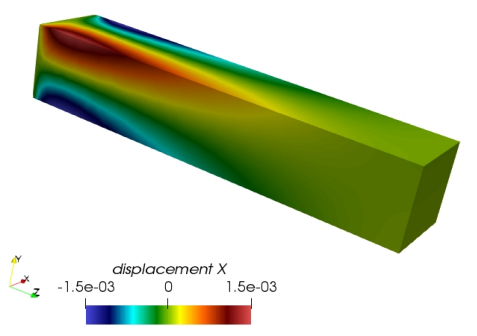

(c) Mean: $x$

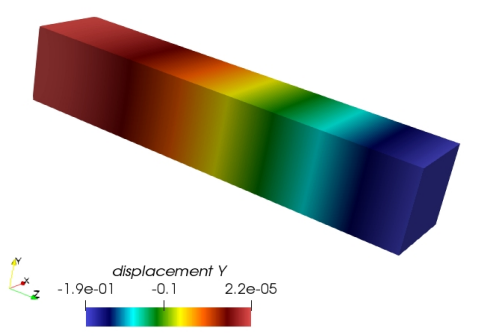

(e) Mean: $y$

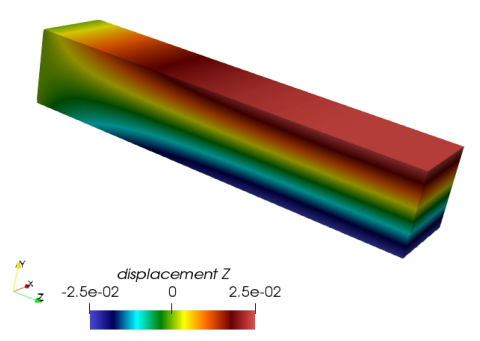

(g) Mean: $z$

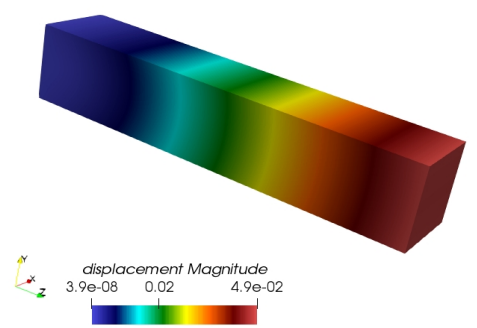

(b) SD: magnitude

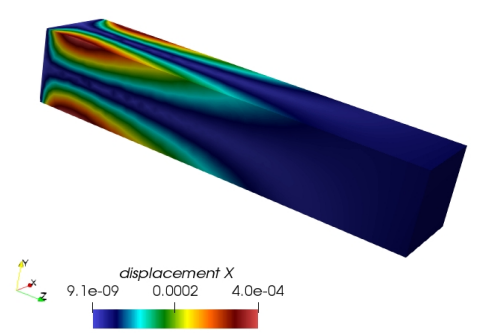

(d) SD: $x$

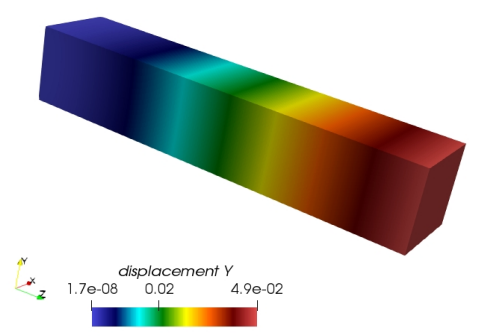

(f) SD: $y$

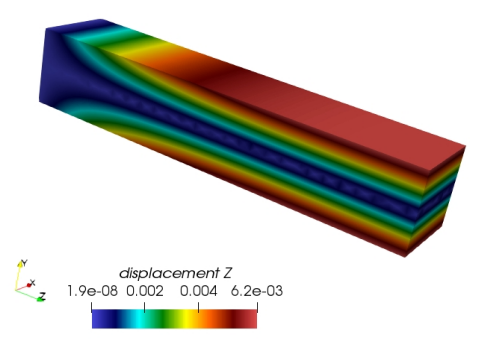

(h) SD: $z$

Figure 4.25: Magnitude and $x, y$ and $z$ components of the mean and standard deviation of the solution process $\mathcal{U}$. 


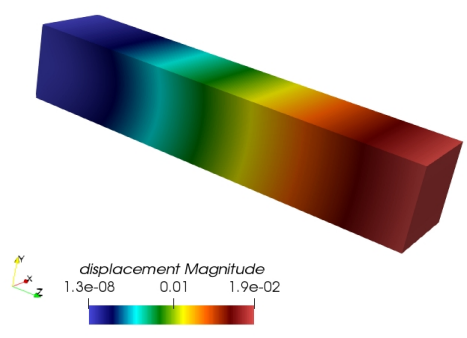

(a) $u_{1}$ : magnitude

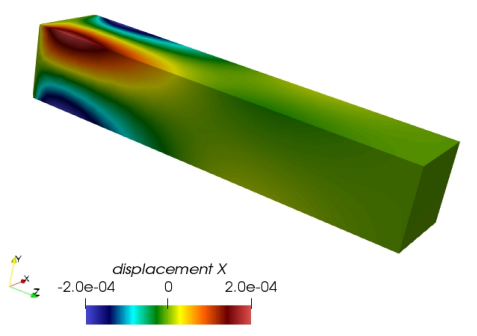

(c) $u_{1}: x$

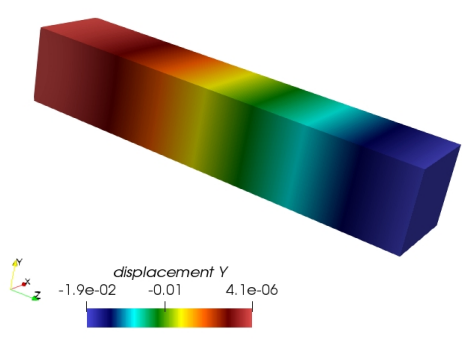

(e) $u_{1}: y$

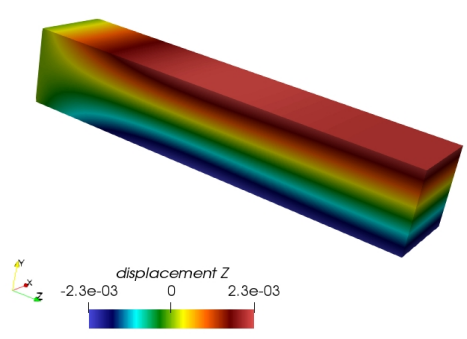

(g) $u_{1}: z$

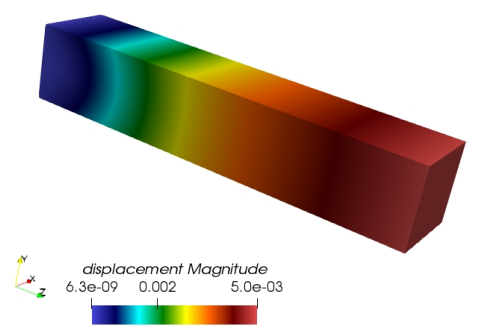

(b) $u_{2}$ : magnitude

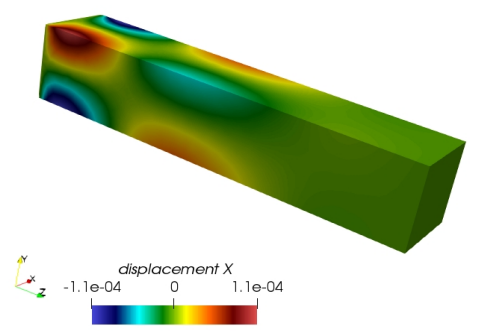

(d) $u_{2}: x$

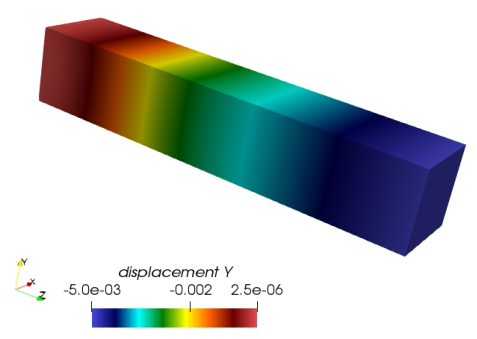

(f) $u_{2}: y$

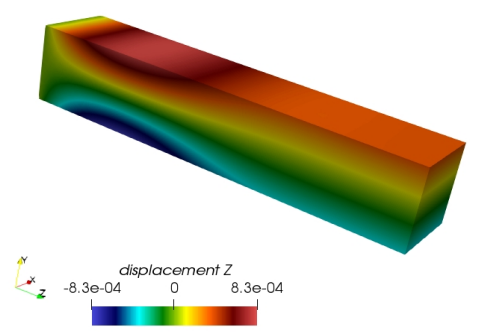

(h) $u_{2}: z$

Figure 4.26: Magnitude and $x, y$ and $z$ components of the selected PCE coefficients of the solution process $\mathcal{U}$. 


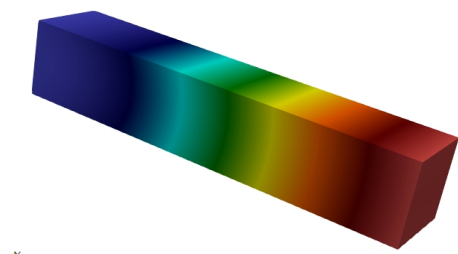

$*_{3}^{*} \begin{array}{cccc}\text { displacement Magnitude } \\ 2.1 \mathrm{e}-09 & 0.002 & 0.004 & 0.1 \mathrm{e}-03\end{array}$

(a) $u_{4}$ : magnitude
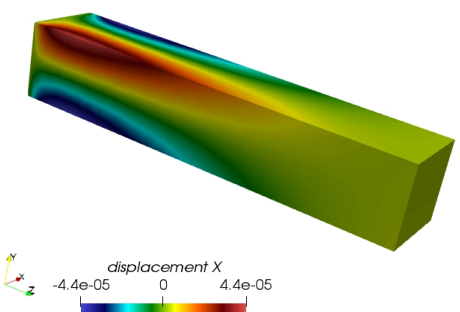

(c) $u_{4}: x$

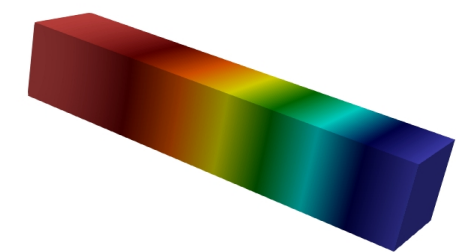

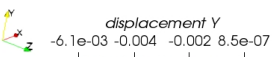

(e) $u_{4}: y$

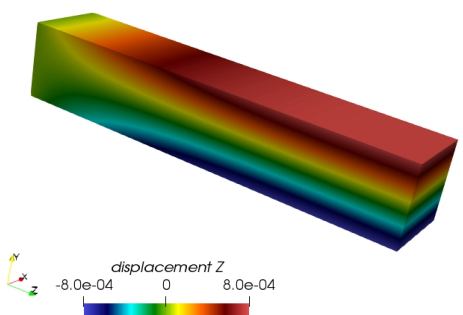

(g) $u_{4}: z$

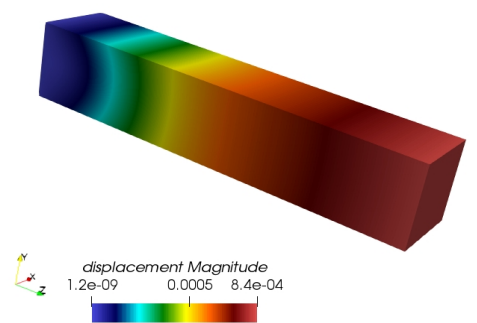

(b) $u_{6}$ : magnitude

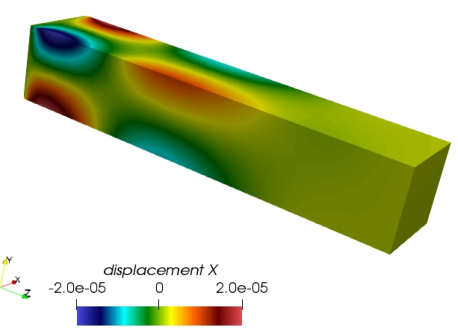

(d) $u_{6}: x$

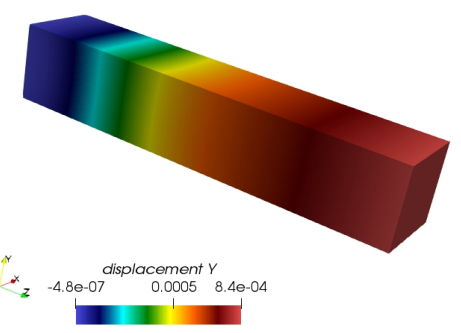

(f) $u_{6}: y$

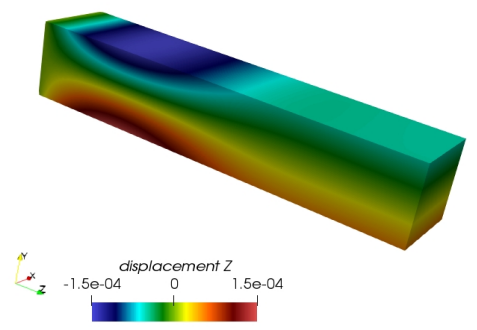

(h) $u_{6}: z$

Figure 4.27: Magnitude and $x, y$ and $z$ components of the selected PCE coefficients of the solution process $\mathcal{U}$. 


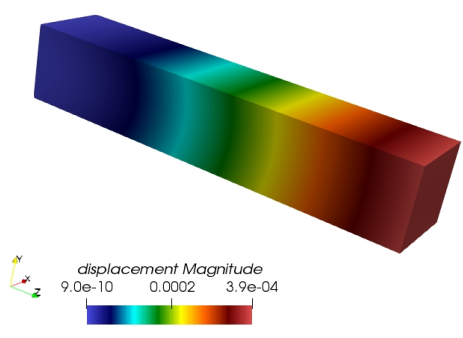

(a) $u_{10}$ : magnitude

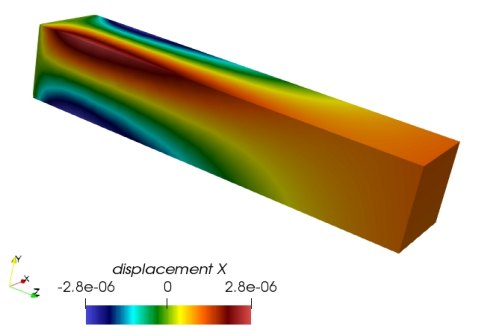

(c) $u_{10}: x$

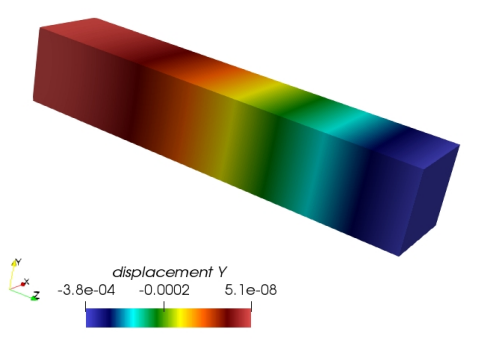

(e) $u_{10}: y$

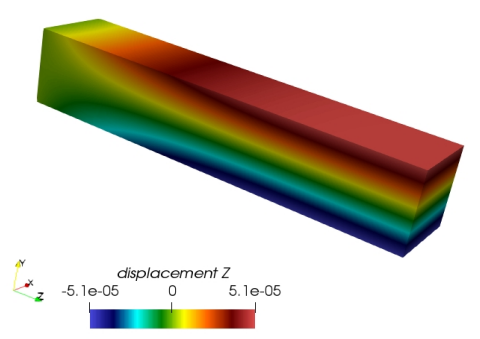

(g) $u_{10}: z$

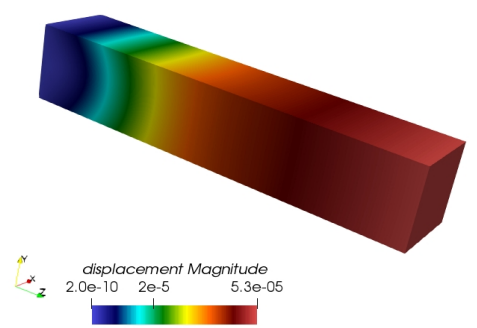

(b) $u_{12}$ : magnitude

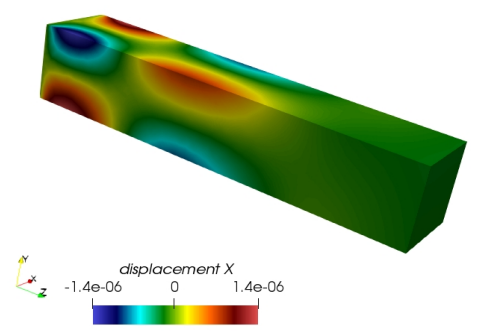

(d) $u_{12}: x$

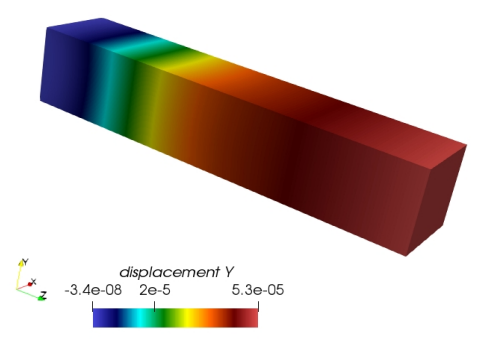

(f) $u_{12}: y$

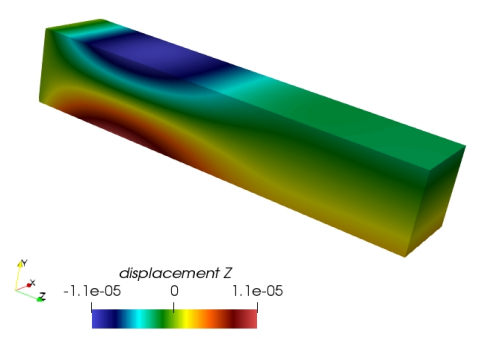

(h) $u_{12}: z$

Figure 4.28: Magnitude and $x, y$ and $z$ components of the selected PCE coefficients of the solution process $\mathcal{U}$. 


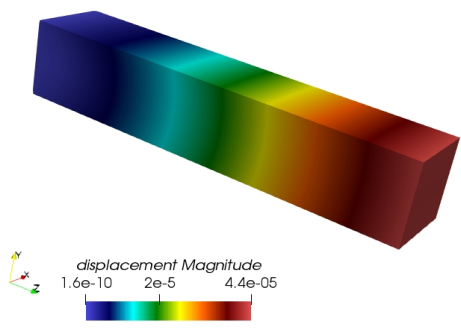

(a) $u_{15}$ : magnitude

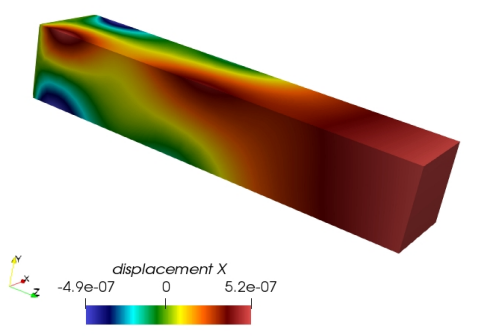

(c) $u_{15}: x$

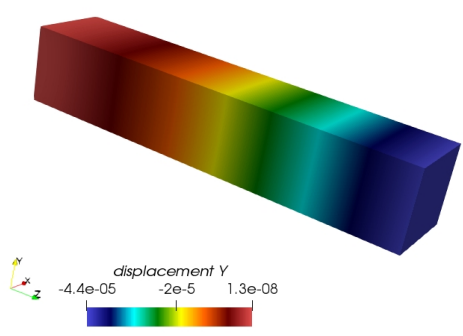

(e) $u_{15}: y$

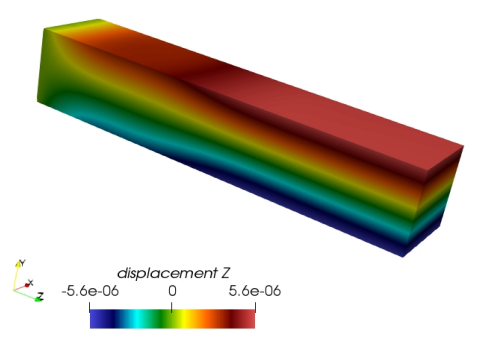

(g) $u_{15}: z$

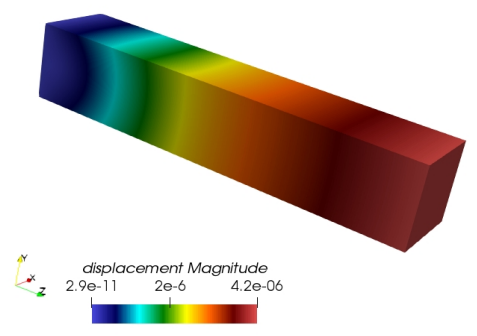

(b) $u_{19}$ : magnitude

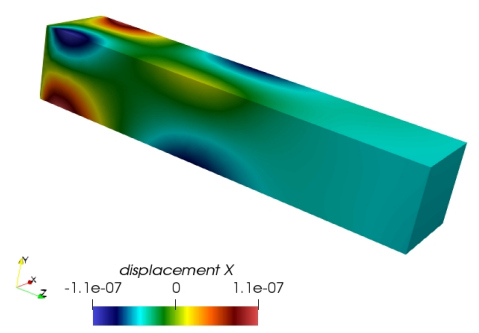

(d) $u_{19}: x$

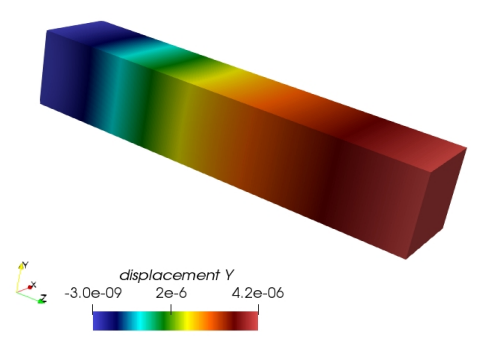

(f) $u_{19}: y$

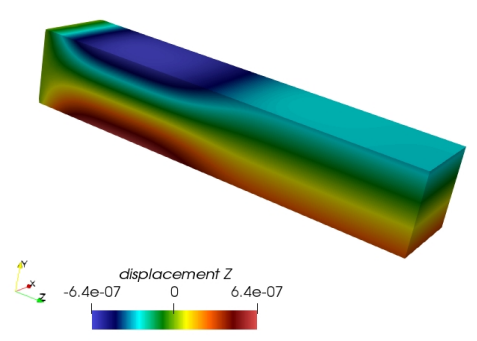

(h) $u_{19}: z$

Figure 4.29: Magnitude and $x, y$ and $z$ components of the selected PCE coefficients of the solution process $\mathcal{U}$. 


\subsubsection{Comparison of Extended Wirebasket with Vertex Coarse Grid}

The performance of the extended wirebasket-based coarse grid preconditioner is compared to the extended vertex-based coarse grid to tackle the stochastic PDE system in linear elasticity. The primary focus is given to the performance comparison concerning numerical scalabilities of the solvers, i.e., the number of PCGM iterations required for the convergence with the tolerance:

$$
\frac{\left\|\mathcal{U}_{\Gamma_{i+1}}-\mathcal{U}_{\Gamma_{i}}\right\|_{2}}{\left\|\mathcal{U}_{\Gamma_{i}}\right\|_{2}} \leq \text { tol }=10^{-5}
$$

with the subscript indicating the PCGM iteration number.

For a fixed mesh resolution, i.e., 31598 nodes and 182681 (four node) linear tetrahedral elements, with a fixed number of PCE terms $\left(P_{u}=56\right.$ for $\left.5 \mathrm{RVs}\right)$, if we increase the number of subdomains the PCGM iteration count grows quickly with the vertex-based coarse grid. The growth of the iteration count with the wirebasket-based coarse grid is smaller compared to that of the vertex-based coarse grid. Fig. 4.30 suggests that the BDDC/NNC solver with wirebasketbased coarse grid showed superior performance in terms of number of PCGM iterations with respect to the number of subdomains compared to the vertex-based coarse grid.

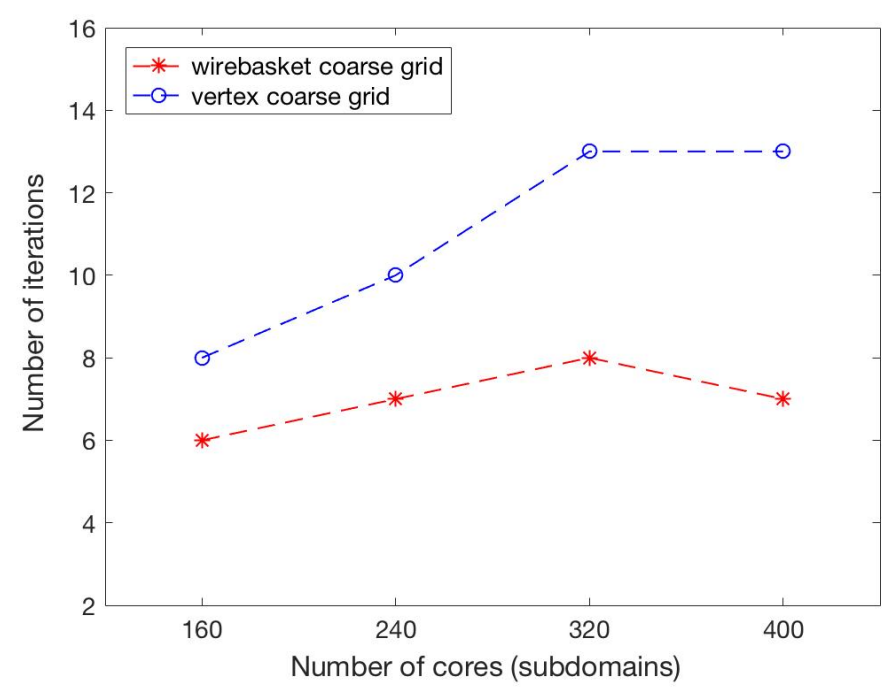

Figure 4.30: Iteration count versus number of subdomains for the fixed mesh resolution with fixed number of PCE terms. 
For a fixed mesh resolution (20753 nodes and 119179 linear tetrahedral element), the global problem size grows with increasing number of PCEs by changing the number of RVs $L=$ $2,3,5$ and 7 . The ensuing larger linear system is then solved using more cores by keeping the total problem size per core approximately the same $(\approx 22500)$. The iteration count for the wirebasket-based BDDC/NNC-PCGM solver versus the number of subdomains (with the fixed problem size per subdomain) increases slightly as shown in Fig. 4.31. On the other hand, for the same case, the number of iterations grows quickly with the vertex-based coarse grid. Therefore, the BDDC/NNC solver with the wirebasket-based coarse grid is superior to the vertex-based coarse grid concerning the numerical scalability with respect to the number of PCE terms.

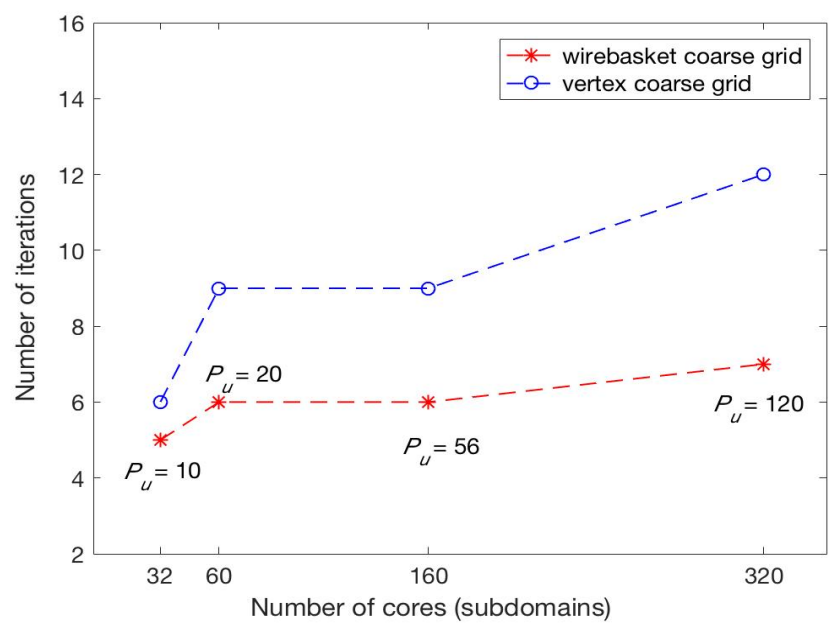

Figure 4.31: Iteration count versus number of subdomains for fixed problem size per subdomain with increasing number of PCEs (fixed mesh resolution).

For the same case as in Fig. 4.31, the total execution time for the BDDC/NNC solver with wirebasket-based coarse grid and vertex-based coarse grid versus the number of subdomains (with the fixed problem size per subdomain) are compared in Fig. 4.32. Total execution time for the BDDC/NNC solver with the vertex-based coarse grid increases faster compared to that of wirebasket-based coarse grid. This is primarily due to more PCGM iteration required with the vertex-based coarse grid.

For a fixed spatial resolution (31598 nodes and 182681 linear tetrahedral element) and fixed number of subdomains $\left(n_{s}=400\right)$, an increase in the number of PCE terms by increasing the 


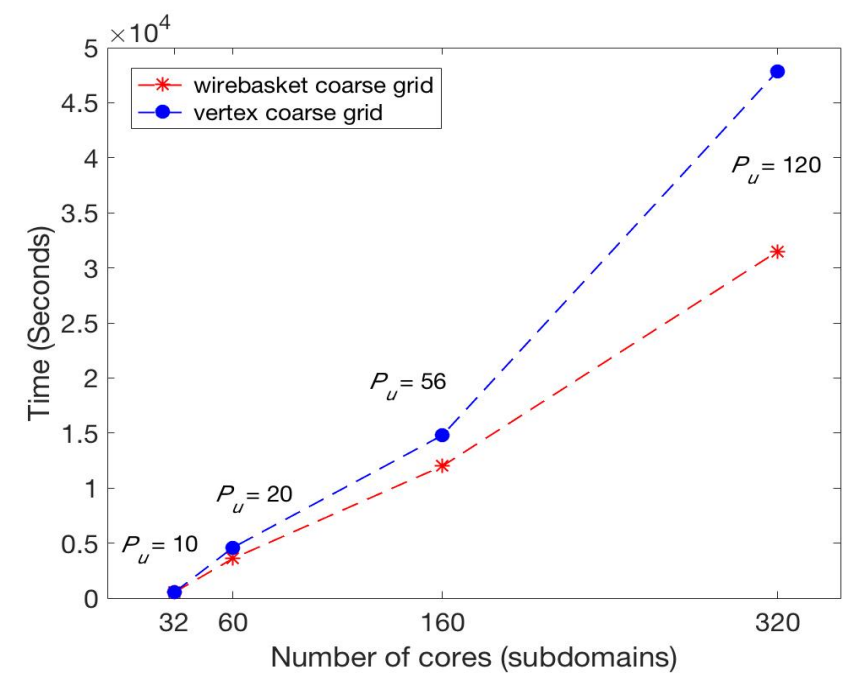

Figure 4.32: Execution time versus number of subdomains for fixed problem size per subdomain with increasing number of PCEs (fixed mesh resolution).

number of random variables (while maintaining the third-order expansion), results in a small increase in the PCGM iteration counts for the case with the wirebasket-based coarse grid, as shown in Fig. 4.33. Conversely, for the same case, the number of iterations increases faster with the vertex-based coarse grid. Therefore, the BDDC/NNC solver with the extended wirebasketbased coarse grid is superior to vertex-based coarse grid concerning number of PCGM iterations with respect to the number of PCE terms and number of subdomains.

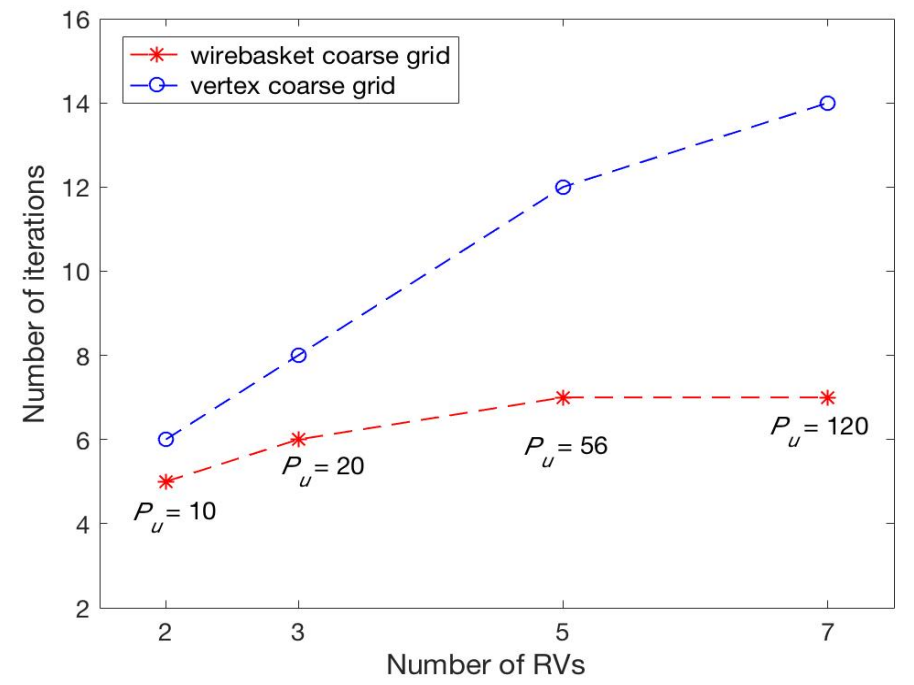

Figure 4.33: Iteration count versus number of PCE terms for the fixed mesh resolution with fixed number of subdomains. 


\subsubsection{Scalabilities Studies for Stochastic Simulations}

The central purpose of this study is to investigate the performance of the proposed BDDC/NNC solver with the wirebasket-based coarse grid for the problems with high dimensions in stochastic space. Therefore, for most of the simulations, the finite element mesh with the fixed number of nodes 48563 and elements 283886 is used. For some cases, the mesh size is increased up to 89657 nodes and 523450 elements. To investigate the high-dimensional stochastic spaces, the performance plots for various cases by selecting the number of random variables ranging between 2 to 9 are presented. These cases are selected based on the relative contribution of the KLE eigenvalues (see Fig. 4.6).

The orders of expansion $p_{\mathrm{A}}=2$ and $p_{u}=3$ are used for the input and output PCE representations, respectively. Note that the orders of expansion, $p_{\mathrm{A}}$ and $p_{u}$ are kept constant for most of the experiments. For some cases $p_{u}$ is varied between 2 to 4 . With these KLE and PCE inputs, the number of PCE terms required to represent the solution process varies from 20 to 220 [1]. The maximum size of the coupled system of linear equations simulated in this case is about 60 million, i.e., using finite element mesh with approximately 90 thousand spatial node points and 220 PCE terms (9-RVs).

In the subsequent sections, first, we study the numerical scalability of the solver concerning the number of outer PCGM iterations required for the convergence with the tolerance tol $=$ $10^{-5}$. Next, we examine the (strong and weak) parallel scalabilities in terms of time against the number of subdomains. Finally, we present the numerical and parallel scalability plots with respect to the stochastic parameters such as the number of random variables and the order of expansion.

\section{Numerical Scalability}

For a fixed problem size (48563 nodes and 283886 elements) with fixed number of PCE terms $\left(P_{u}=56\right)$, if we increase the number of subdomains, the PCGM iteration count increases slightly. Fig. 4.34 indicates that the BDDC/NNC-PCGM solver with the wirebasket-based 
coarse grid is numerically scalable with respect to the number of subdomains.

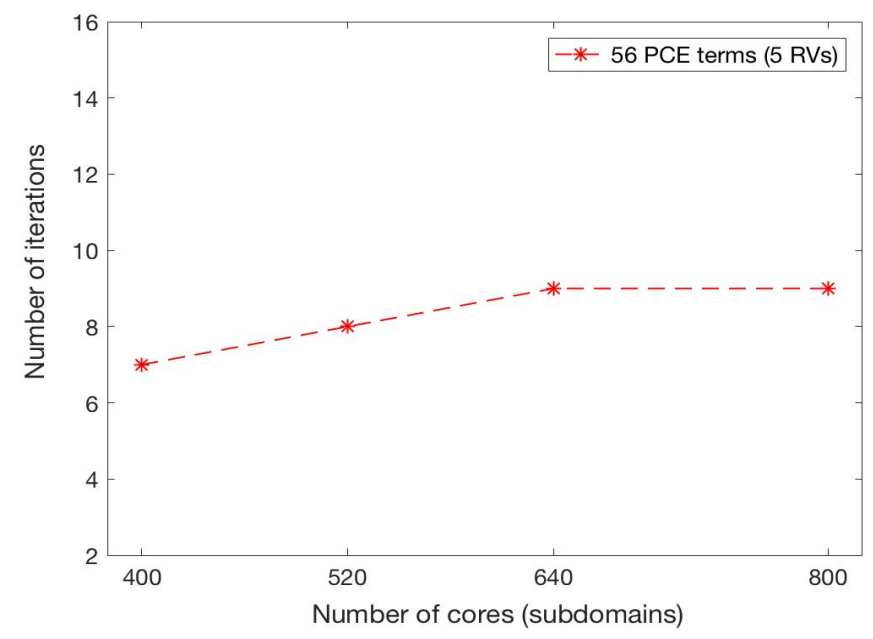

Figure 4.34: Iteration count versus number of subdomains for the fixed mesh resolution with fixed number of PCE terms.

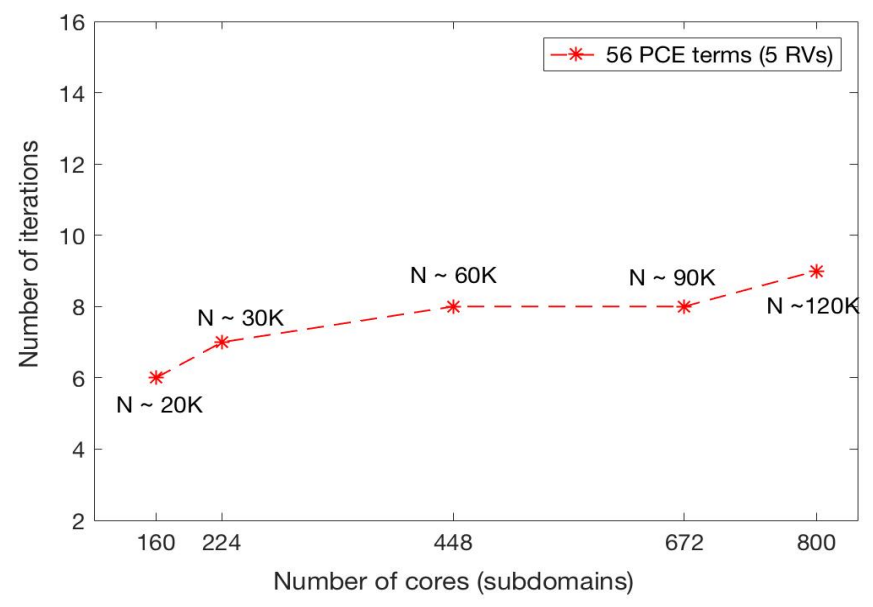

Figure 4.35: Iteration count versus number of subdomains for the fixed problem size per core with increasing mesh resolution (fixed number of PCE terms).

Next, for the fixed number of PCE terms $\left(P_{u}=56\right)$, the global problem size is increased by increasing mesh resolution while keeping a fixed problem size per subdomain $(\approx 22500)$. As shown in Fig. 4.35, the PCGM iteration count increases slowly. Fig. 4.35 indicates that the wirebasket-based BDDC/NNC solver show better numerical performance concerning mesh resolution and fixed problem size per subdomains. 


\section{Parallel Scalabilities}

The strong scalability of the BDDC/NNC-PCGM solver is plotted in Fig. 4.36. For fixed spatial mesh resolution (48563 nodes and 283886 elements) with the fixed number of PCE terms ( $P_{u}$ $=56$ ), an increase in the number of cores reduces the execution time as expected. Fig. 4.36 indicates excellent strong scaling of the BDDC/NNC solver with the vertex based coarse grid.

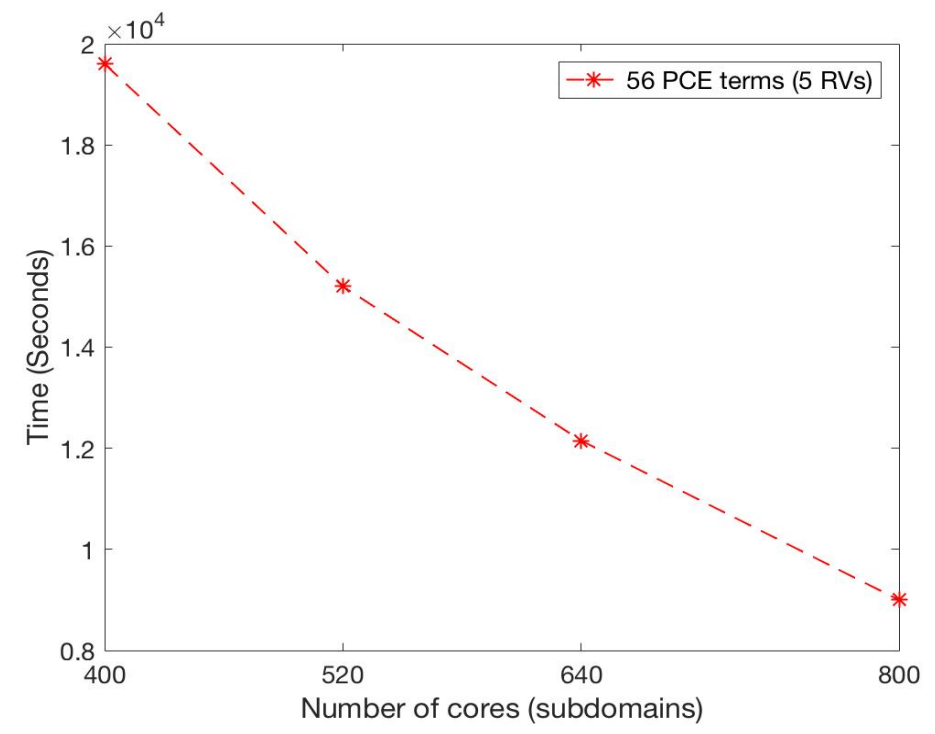

Figure 4.36: Execution time versus number of subdomains with the fixed mesh resolution and the number of PCE terms.

The weak scalability of the BDDC/NNC-PCGM solver is plotted in Fig. 4.37. For the fixed number of PCE terms $\left(P_{u}=56\right)$, the global problem size is increased by increasing mesh resolution while keeping a fixed problem size per subdomain $(\approx 22500)$. The growth in the total execution time implies that the probabilistic BDDC/NNC shows suboptimal weak scalability. This is mainly because of the increased parallel overhead with the number of cores caused by the MPI collective communication [19,24]. Also, in the current case, the size of the global coarse problem grows quickly with the increasing number of subdomains due to vector-valued solution process. Consequently, the resulting coupled system of equations is computationally challenging. Therefore, it demands significantly more computational efforts compared to that of scalar-valued stochastic PDEs. 


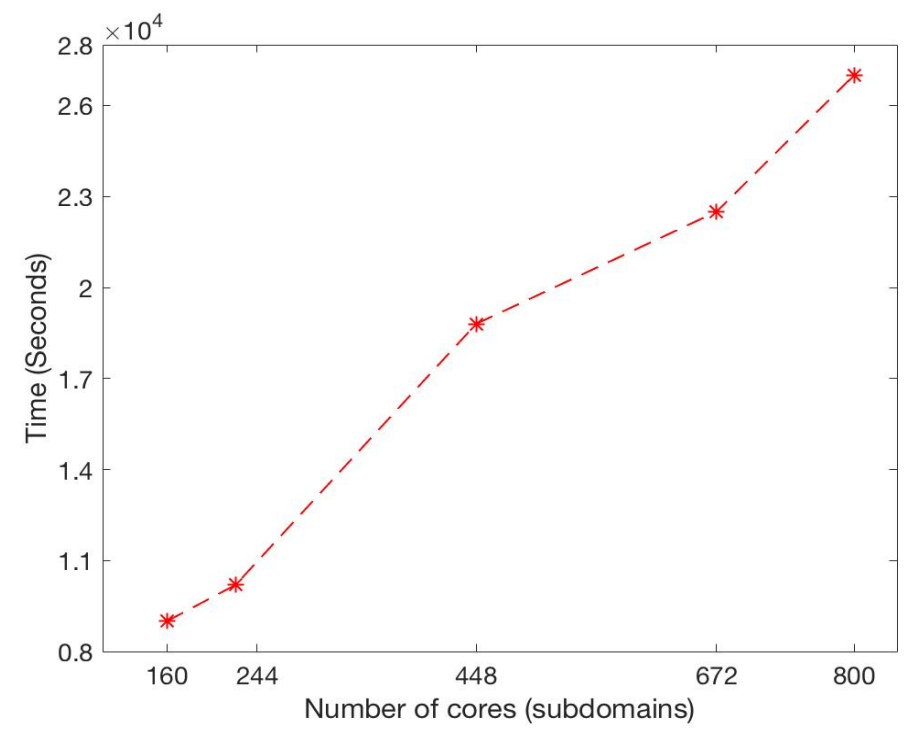

Figure 4.37: Execution time versus number of subdomains for the fixed problem size per core with increasing mesh resolution (fixed number of PCE terms).

\section{Scalability with respect to Stochastic Parameters}

To examine the effects of stochastic aspects on the scalabilities of the solvers, the numerical and parallel scalabilities with respect to stochastic parameters such as the number PCE terms and the order of expansions are presented in this section. The four points in Fig. 4.38 and 4.39 corresponding to the number of PCE terms 20,56, 120 and 220 are related to the number of random variables 3, 5, 7 and 9 respectively. The finite element mesh (spatial) resolution corresponds to 48563 nodes and 283886 elements.

For a fixed spatial resolution and fixed number of subdomains $\left(n_{s}=800\right)$, an increase in the number of PCE terms by increasing the number of random variables (while maintaining the third-order expansion) results in a slight variation in the PCGM iteration counts, as shown in Fig. 4.38. This result indicates that the wirebasket-based BDDC/NNC-PCGM solver showed good numerical scaling with respect to the number of random variables. Although the total problem size increases with the number of PCE terms, one can solve the larger system with about the same number of PCGM iterations.

With the increasing number of PCEs, the global problem size increases for a fixed mesh reso- 


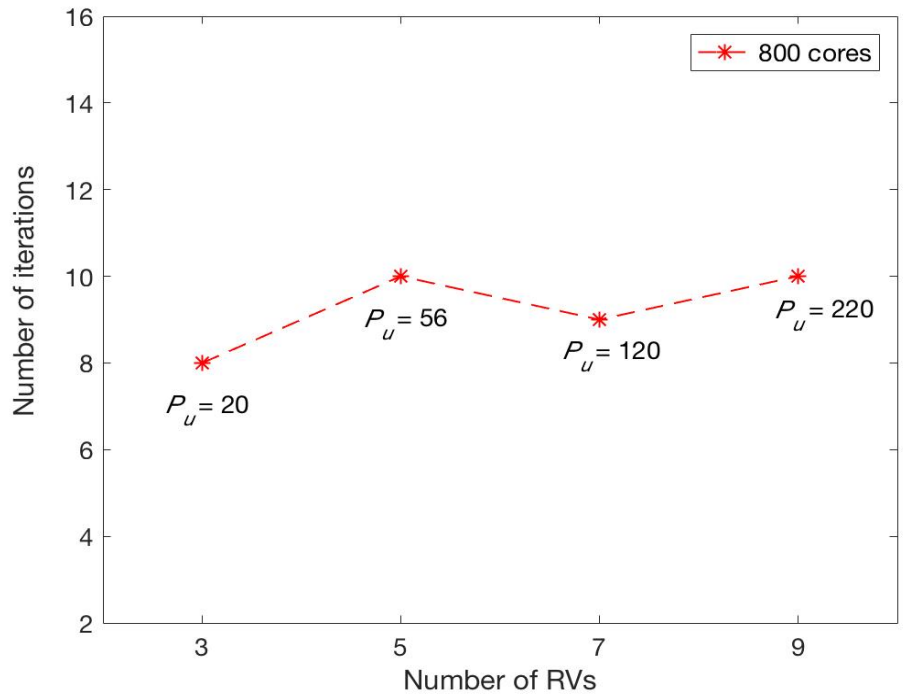

Figure 4.38: Iteration count versus number of PCE terms for the fixed mesh resolution with fixed number of subdomains.

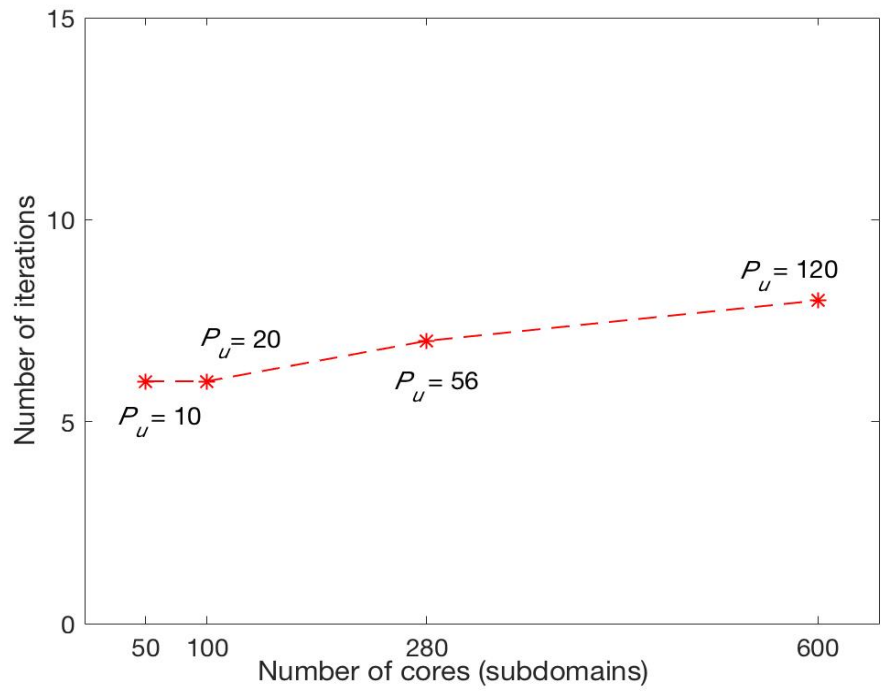

Figure 4.39: Iteration count versus number of subdomains for the fixed problem size per core with increasing number of PCE terms (fixed mesh resolution). 


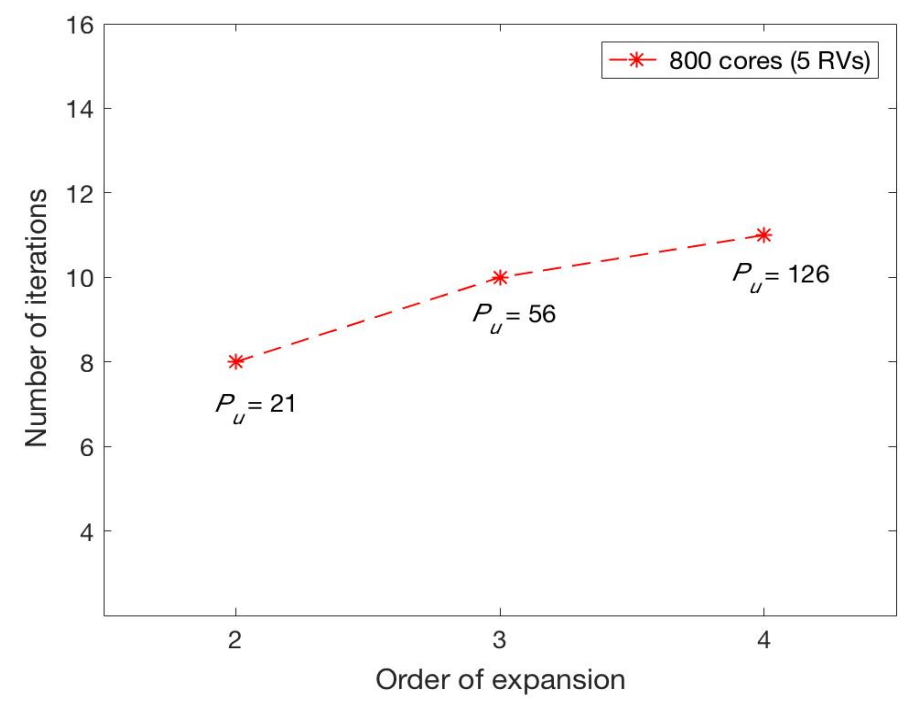

Figure 4.40: Iteration count versus order of expansion for the fixed mesh resolution with fixed number of subdomains (fixed number of RVs).

lution. The ensuing larger linear system is then solved using more cores by keeping the problem size per core approximately the same $(\approx 18000)$. The iteration count for the BDDC/NNCPCGM solver versus the number of subdomains (with the fixed problem size per subdomain) remains constant as shown in Fig. 4.39. Hence the wirebasket-based BDDC/NNC-PCGM solver showed acceptable numerical scaling with respect to the number of PCEs.

For a fixed mesh resolution and fixed number of subdomains $\left(n_{s}=800\right)$, an increase in the number of PCE terms by increasing the order of expansion $p_{u}$ (while maintaining the fixed number of random variables $L=5$ ) results in moderate increase in the PCGM iteration counts as shown in Fig. 4.40. This result indicates that the wirebasket-based BDDC/NNC-PCGM solver showed satisfactory numerical scaling with respect to the order of expansion.

In summary, building on the formulations of wirebasket-based coarse grid [25, 26, 209, 210] for deterministic PDEs, this work devised the formulation of the extended wirebasketbased coarse grid for the stochastic PDEs. The BDDC/NNC-PCGM solver with the extended wirebasket-based coarse grid is employed to tackle three-dimensional (scalar and vector-valued) stochastic PDEs with high-dimensional stochastic spaces. The novel domain decomposition preconditioner based on the extended wirebasket-based coarse grid is shown to outperform 
vertex-based coarse grid for stochastic PDEs in three dimensions. The stochastic system matrix assembly procedure designed to utilize existing deterministic FEM packages reduces the implementational burden of DD-based intrusive SSFEM. Various sparse data structures, routines and iterative solver with efficient preconditioners are utilized to tackle the subdomain-level systems. These implementational advances reduce the execution time and memory consumption; hence offer the ability to solve extreme-scale stochastic systems using large-scale HPC clusters. Numerous simulations are performed to thoroughly investigate the performance of the extended wirebasket-based BDDC/NNC solver to tackle large-scale high-dimensional stochastic PDEs. The scalability plots provided for numerical and parallel scaling with respect to the number of subdomains and the number of PCE terms are essential in understanding convergence behavior and utility of the solver for uncertainty quantification of large-scale practical applications.

Although extended wirebasket based coarse grid showed better performance for the elliptic stochastic PDEs considered here, the size of the extended coarse problem arising in this setting grows rapidly with (a) the mesh resolution, (b) the number of PCE terms and (c) the number of subdomains. Therefore, the solution of a coarse problem becomes computationally challenging concerning memory and floating point operations. This can be alleviated, up to a certain extent, by extending the recently proposed algorithms in the deterministic setting which utilizes only average constraints over selected edges [216,217]. In this setting, only edge averages over few selected edges are used, therefore, no vertex constraints are required. The numerical experiments performed on deterministic PDEs concludes that the implementation of the primal edge average constraints using a transformation of basis leads to a scalable solver [216,217]. Extending this algorithm in the stochastic setting could substantially reduce the size of the coarse problem compared to the wirebasket grid and therefore can improve the performance of the DD-based solvers for stochastic PDEs. 


\section{Chapter 5}

\section{Summary of Research Findings and Future Directions}

This chapter summarizes the major findings, provides directions for future extensions and concludes the thesis. The chapter is organized in the following manner: First, the summary of research findings is presented in Sec. 5.1. Next, in Sec. 5.2, limitations of the current study are discussed. This is followed by the possible direct extensions and future research directions in Sec. 5.3. Finally, in Sec. 5.4, we conclude the thesis.

\subsection{Summary of Research Findings}

In this thesis, scalable parallel DD-based solvers for SSFEM are devised for uncertainty quantification of large-scale discretized elliptic stochastic PDEs with a large number of random variables. With the motive to alleviate the curse of dimensionality in SSFEM, the primary focus of the investigation is given to the algorithmic developments and efficient HPC implementation of the solvers for intrusive SSFEM. The framework is based on (a) the non-overlapping domain decomposition in the spatial domain discretized using finite element methods and (b) a functional expansion using polynomial chaos is utilized in the stochastic domain. A threelevel nested parallel preconditioned conjugate gradient methods, employing an efficient pre- 
conditioner at each level, are devised to solve extended Schur complement system. The nested iterative solvers employed for the solution of two-dimensional stochastic PDEs using intrusive SSFEM are equipped with two-level preconditioner accompanied by the vertex-based coarse grid. For the cases of three-dimensional stochastic PDEs, the nested PCGM solver with the two-level preconditioner having a wirebasket-based coarse grid is developed.

The DD algorithms for intrusive SSFEM are implemented using (a) MPI for parallel communications, (b) FEniCS for FEM discretization and subdomain-level deterministic system assembly and (c) PETSc for subdomain-level stochastic system assembly and linear algebraic calculations. The numerical simulations are performed on a two-dimensional and three-dimensional stochastic PDEs with non-Gaussian random system parameters. The performance results for two-dimensional stochastic diffusion equation demonstrate that the probabilistic versions of BDDC/NNC and FETI-DP solvers with the vertex-based coarse grid are numerically scalable concerning the number of subdomains and the number of random variables. The performance plots for three-dimensional stochastic PDEs (diffusion equation and equations of linear elasticity) demonstrate that the probabilistic version of BDDC/NNC solver equipped with the wirebasket-based coarse grid showed better numerical scaling concerning the number of subdomains and the number of random variables. Furthermore, the advances made in both algorithmic and HPC implementational aspects enable the DDM based intrusive SSFEM approach to outperform the commonly used sparse grid based non-intrusive SSFEM approach for high-dimensional stochastic PDEs with non-Gaussian random variables. The implementational challenges of the intrusive SSFEM due to the necessity of intrusive adjustments in the governing PDE, are addressed using FEniCS based deterministic assembly routines. The use of FEniCS simplifies the applications of the domain decomposition based intrusive SSFEM to tackle a variety of PDEs. The further details on research findings are summarized as follows:

(1) The probabilistic BDDC/NNC and FETI-DP solvers formulated in [17, 18] are extended in both algorithmic and implementation aspects to solve large-scale discretized stochastic PDEs with a large number of random variables. The three-level sparse iterative solvers devised by em- 
ploying an efficient an efficient preconditioner at each level are implemented using objects and routines from MPI, PETSc, FEniCS, and UQTk [19]. The algorithmic and implementational advancements allow the solvers to scale up to 25 random variables and provide the capabilities to tackle the intrusive SSFEM system with 216 million unknowns using 1680 computing cores. Therefore, the new solvers alleviate the curse of dimensionality in SSFEM and justify the applicability of the intrusive approach to the large-scale engineering applications.

(2) The probabilistic BDDC/NNC and FETI-DP solvers using vertex based coarse grid developed in $[17,18]$ does not scale for three-dimensional stochastic PDEs [24]. Therefore, the extended wirebasket-based coarse grid is developed to overcome scalability issues of the vertexbased coarse grid in the cases of three dimensional stochastic PDEs. The new coarse grid provides an efficient mechanism for global error propagation and improves the condition number of the preconditioned Schur-complement system. Therefore, this fact enhances the scalability of the probabilistic BDDC/NNC solvers to tackle three-dimensional stochastic PDEs.

(3) In the context of DD-based intrusive SSFEM solvers for the cases involving a large number of random variables, an effective procedure for subdomain level stochastic system matrix assembly is developed. The new local assembly routine optimizes the memory management via the recursive construction of the subdomain level sparse blocks of the stochastic finite element matrices using the subdomain level blocks of deterministic finite element matrices. Using this stochastic assembly procedure, the DD-based intrusive SSFEM solvers are coupled with the FEniCS, a general purpose deterministic finite element package. The coupling with FEniCS enables the DD-based parallel solver to tackle a variety of PDEs. Therefore, it lessens the burdens involved in the code development process for the intrusive $\operatorname{SSFEM~[9,106,108].~Furthermore,~}$ the FEniCS assembled deterministic blocks are also utilized to preallocate memory for PETSc stochastic blocks matrices. This helps to further optimize memory allocation and improve the solver capabilities to handle large-scale applications.

(4) Scalable and efficient implementation of the proposed solvers concerning stochastic dimensions is accomplished by consolidating the following features. First, the solvers utilize 
various sparse data-structures and optimized routines from PETSc for commonly occurring subdomain level algebraic operations such as local matrix-vector product and local system solves in multi-level PCGM implementations. Second, the moments of multidimensional polynomials which require thousands of multidimensional inner products, are obtained using routines developed by adapting procedures from UQ Toolkit involving strictly one-dimensional moments. Third, the computations of large sets of multidimensional PCE/KLE basis functions and the calculation of input PCE/KLE coefficients are automated to scale for large number of random variables, using the routines developed by adapting functions from UQ Toolkit.

(5) The performance comparison of intrusive SSFEM against non-intrusive SSFEM is presented for non-Gaussian random system parameters with increasing number of random variables. This was lacking in the numerous assessments performed in the past $[101,113,118]$, which dealt with the uniform or Gaussian random inputs. A comprehensive error analysis of the individual PCE coefficients of the solution process is conducted to obtain further insights into the convergence behavior of both intrusive and non-intrusive approaches. Furthermore, using the DD-based solver for intrusive SSFEM, efficiently implemented using MPI and PETSc libraries, is shown to outperform the commonly used sparse grid based non-intrusive SSFEM for high-dimensional stochastic PDEs. This fact, emphasizes the significance of the scalable parallel algorithms and their efficient HPC implementation for uncertainty propagation in large-scale engineering systems.

(6) This thesis elaborates on the HPC implementational aspects of the solvers and their scalability studies on large-scale HPC clusters with thousands of cores. This was lacking in the previous articles related to these methods $[17,18]$. The algorithmic details and code snippets are presented for completeness to assist computational scientists in implementing these methods for their applications [19]. As a result, these developments will pave the way for the new researchers in this field to apply these solvers to various other engineering applications. The detailed performance analysis and scalability studies are performed on the large-scale computing clusters with up to 4000 computing cores. To the best knowledge of the author, these algo- 
rithms have not been applied for the cases of a considerable number of random variables and never been tested on a large-scale computing cluster at the level which is accomplished in this work.

From an application perspective, the DDM solvers for intrusive SSFEM, coupled with the FEniCS general purpose FEM package, can be leveraged for uncertainty quantification involving a variety of stochastic PDEs. As a demonstration, in this thesis, the solvers are employed to tackle the commonly used diffusion equation and linear elasticity problem in three dimensions. Therefore, this thesis demonstrates the applicability of the solvers to the scalar and vectorvalued stochastic PDEs. Also, the capability of the solvers to model non-Gaussian material properties exploiting UQTk makes them more useful for practical applications. Furthermore, the scalable DDM solvers for SSFEM, are combined with an in-house Bayesian estimation package for large-scale data assimilation problems [21, 178]. The DDM solvers are used as the forecast (prediction) step to propagate the PCE coefficients of the state efficiently [21, 178]. When sensor data is available, the PCE coefficients are updated using a parallel update (analysis) step [21, 178] of the polynomial chaos Kalman filter [20,21, 178].

\subsection{Limitations of the Study}

In this thesis, the scalable parallel DD-based solvers for intrusive SSFEM are demonstrated to tackle large-scale discretized stochastic PDEs in two and three dimensions with a large number of random variables. Although these solvers exhibit excellent numerical and parallel scaling, there are some limitations need to be addressed to further enhance the applicability of these solvers. The list of challenges is outlined as follows:

(1) The poor weak scaling of the DD-based SSFEM solver, i.e., the growth in the total execution time concerning mesh resolution for a fixed problem size per subdomain and a fixed number of PCE terms. This suboptimal weak scaling of the solver, observed in both two and three-dimensional cases, is primarily because of the increased parallel overhead with the num- 
ber of subdomains due to MPI collective communication in the current implementation [19, 24]. Furthermore, the size of the global coarse problem grows with the increasing number of subdomains, therefore, further affects the execution time. These challenges can limit the maximum size of the intrusive SSFEM system that can be solved using this solver in the current implementational setting.

(2) The poor parallel scaling is observed concerning the number of PCE terms, (i.e., an increase in the execution time with respect to the PCE terms for a fixed problem size per subdomain and fixed mesh resolution). This can be attributed to (a) MPI collective communication used in the current implementation, leading to increased parallel overhead with the increase in the number of cores, and (b) the increased number of PCE terms lead to a more extensive system of coupled equations, and the new system inherits a more complex coupling structure. The initial investigation suggests that the new coupling structure influences the condition number of the system; therefore demands more computational effort. This can also limit the maximum size of the intrusive SSFEM system that can be solved using this solver.

(3) The size of the extended coarse problem arising in the application of two-level DD methods to the three-dimensional stochastic PDEs grows rapidly with (a) the mesh resolution, (b) the number of PCE terms and (c) the number of subdomains. This is more so in the case of the extended wirebasket-based coarse grid due to the inclusion of interface-edges. Therefore, the solution of a coarse problem becomes computationally challenging concerning memory requirement and execution time. In the current implementation, the probabilistic coarse problem arising in the setting of two-level preconditioner is solved using the one-level lumped preconditioner. This can limit the application of DD-based solver to extreme-scale stochastic PDEs using a very large number of subdomains due to the poor convergence of the one-level preconditioner. 


\subsection{Future Research Directions}

To overcome the above-listed limitations of this research and to further improve the performance of the DD-based solvers for intrusive SSFEM presented in this thesis, the author envisions following research directions.

\subsubsection{Direct Extensions of the Study}

(1) The poor weak scaling with number of subdomains and poor parallel performance with the number of PCE terms are observed because, the parallel overhead increases with the number of subdomains. This is mainly due to the parallel implementation using MPI collective communication $[19,24]$. This can be alleviated in the future implementation of these solvers by using MPI point-to-point communication routines, such as MPI_send and MPI_receive, whenever applicable [33]. Furthermore, the communicator-aware and interlevel-overlapped messagepassing implementation can be employed using MPI sub-communicators as suggested in [207]. These changes show promising results for DD-based solvers in deterministic setting [201,207]. Such developments can be utilized in the future implementation of these DD-based solvers in the stochastic setting.

(2) The increased number of PCE terms leads to a more extensive system of coupled equations, and the new system inherits a more complex coupling structure, therefore, demands more computational effort. In the current implementation, the local systems are solved using PETScPCGM with PETSc built-in block-Jacobi preconditioner. This is effective but computationally expensive for an extremely large system of linear equations. In the future implementations of the solvers, one can use more sophisticated preconditioners tailored to exploit stochastic coupling; such as Kronecker product preconditioner [154] or hierarchical Schur complement preconditioner [155]. This can further expedite the convergence of the subdomain level interior problems and reduce the total execution time. 
(3) In the context of two-level DD-based solvers for three-dimensional stochastic PDEs, the growing size of the global coarse problem with the increasing number of PCE terms and number of subdomains can be handled by doing following enhancements:

- An algebraic multigrid preconditioner for the coarse problem can be formulated to replace the one-level lumped preconditioner used in the current implementation.

- In deterministic settings, the increased size of the coarse problem is managed using efficient scalable iterative solvers [26,218,219]. A similar approach can be exploited in the stochastic settings.

- A reduced-order probabilistic coarse problem can be formulated using the stochastic reduced basis methods $[84,173,220]$. These advances can further improve the computational efficiency of the solver.

\subsubsection{Broader Issues to be Covered in Future Work}

(1) For a fixed mesh with the fixed number of subdomains, as the number of random variables increases, the computational burden on each processor increases. Consequently, this fact increases the total execution time for probabilistic domain decomposition solvers for SSFEM. This can be alleviated, to a certain extent, by exploiting two-level concurrency either by using (a) MPI/OpenMP parallelization or by using (b) CPU/GPU parallelization. In the first case, the MPI can be used for inter-processor communications as utilized in the current implementation and the OpenMP can be used to exploit intra-processor concurrency to accelerate the subdomain level computation using multithreading [221,222]. In the second case, rapidly emerging GPU can be exploited. A case study on GPU-based methods for UQ in computational mechanics performed in [222] show significant gains in computational efficiency. The DD-based solver for SSFEM can be adapted to utilize hybrid CPU/GPU clusters to exploit data and task parallelization. Here the data parallelization can be achieved by using MPI on the CPU and the subdomain-level computations can be accelerated using the GPU on that computing 
node [174,222]. Therefore, exploiting GPU could considerable enhance in the computational performance of the DD-based solvers.

(2) An increasing coarse problem size and the subdomain-level system size can limit the total size of the system that can be solved using the DD-based solver devised in this thesis. To overcome this bottleneck, the solver can be adapted to employ more advanced PETSc builtin preconditioners [31]. For instance, the DD-based one-level lumped preconditioner used for coarse problem and subdomain level problems can be replaced by PETSc-based multigrid preconditioner [31]. Likewise, the matrix-free and approximate inverse methods available in the PETSc can also be exploited to further enhance the computational efficiency of the DD-based solvers for stochastic PDEs.

(3) Similar to that of deterministic PDEs, a theoretical analysis of condition number bound for the probabilistic DD-based preconditioners for stochastic PDEs can be performed. The framework for the theoretical analysis can be formulated as [24],

$$
k\left(M^{-1} S\right) \leq f\left(h, H, L, P_{\mathrm{A}}, P_{u}\right)
$$

where $h$ is the finite element mesh size, $H$ is the subdomain size, $L$ is the number of random variables, $P_{\mathrm{A}}$ is the number of PCE terms for the input and $P_{u}$ is the number of PCE terms for the output. In the deterministic setting, only $h$ and $H$ are considered; however, for the stochastic setting, we also need to consider $L, P_{\mathrm{A}}$ and $P_{u}$. This is because $L, P_{\mathrm{A}}$ and $P_{u}$ influence the size and coupling structure of the global and subdomain-level local system matrices and their effects are different in terms of resulting coupling structures (recall Fig. 2.6 and Fig. 2.7). Therefore, these parameters could influence the condition number of the stochastic system matrices arising in the context of intrusive SSFEM. Furthermore, the local KLE basis functions can be employed to reduce the total number of PCE terms. This can lead to a reduced Galerkin problem [175,223]. Also, an optimally efficient stopping criterion to terminate PCGM iteration can be exploited as suggested in [172] to reduce unnecessary computations. 


\subsection{Conclusions}

In this thesis, scalable DD-based parallel iterative solvers are developed and efficiently implemented to solve large-scale discretized stochastic PDEs with a large number of random variables. The three-level nested PCGM solvers, employing an efficient preconditioner at each level are devised to solve the extended Schur complement system using only subdomain-level local system matrix blocks. The local or the global Schur complement matrices were never explicitly assembled. These solvers for SSFEM with intrusive PCE are implemented using objects and routines from scientific computing libraries on high-performance computing clusters. The solvers employed for the solution of two-dimensional stochastic PDEs are equipped with twolevel scalable domain decomposition preconditioner accompanied by the vertex-based coarse grid. For the cases of three-dimensional stochastic PDEs, the nested PCGM solver with the two-level preconditioner having a wirebasket-based coarse grid is developed. These solvers are employed for the numerical simulations of scalar and vector-valued stochastic PDEs with a large number of random variables to alleviate the curse of dimensionality issue in SSFEM.

The performance results for two-dimensional stochastic PDEs demonstrate that the probabilistic versions of BDDC/NNC and FETI-DP solvers with the vertex-based coarse grid are numerically scalable concerning the number of subdomains and the number of random variables. The performance plots for three-dimensional stochastic PDEs demonstrate that the probabilistic version of BDDC/NNC solver equipped with the wirebasket-based coarse grid showed better numerical scaling concerning the number of subdomains and the number of random variables. Such scalabilities are essential to increase the capabilities of the solvers for uncertainty quantification in extreme-scale computational environments.

The parallel implementations of the algorithms developed in this thesis, achieve an excellent strong scaling behavior and sub-optimal scalability in the weak sense. Further investigation is warranted for detailed performance analysis and fine-tuning of the parallel implementation of the probabilistic DDM solvers in both two and three dimensions. The memory usage of the solvers for uncertainty propagation with large stochastic dimensions, highlights the necessity of 
efficient memory management using sparse objects and routines. The total floating-point operations required in the cases of large number of random variables reached the scale of petaflop ( $10^{15}$ floating-point operations). These results emphasize the necessity of large number of cores for large-scale SSFEM systems. A further increase in the number of random variables could demand a computing cluster capable of $10^{18}$ floating-point operations, pointing to emerging exascale computing $[165,166]$. Furthermore, the scalable DD-based solvers for intrusive SSFEM are shown to outperform the non-intrusive SSFEM approach with sparse grid quadrature, thereby substantiating the usefulness of the intrusive SSFEM based scalable solvers. To conclude, the results presented here highlight that the intrusive SSFEM approach combined with probabilistic DDM solvers and high-performance computing is promising tool for extreme-scale uncertainty quantification. 


\section{Appendix A}

\section{Spectral Representation of Stochastic}

\section{Process}

\section{A.1 Karhunen-Loève Expansion}

Consider $\alpha(\boldsymbol{x}, \boldsymbol{\xi}(\theta))$ to be a real-valued stochastic process, a function of the position vector $\boldsymbol{x}$ defined over physical domain $\mathscr{D}$ and the set of random variables $\boldsymbol{\xi}$ which are a function of random event $\theta$ defined by complete probability space $(\Omega, \mathscr{E}, \mathscr{P})$. The KL expansion of an arbitrary non-Gaussian and non-stationary stochastic process using $L$ random variables can be written as $[1,2]$,

$$
\alpha(\boldsymbol{x}, \boldsymbol{\xi}(\theta))=\bar{\alpha}(\boldsymbol{x})+\sum_{n=1}^{L} \sqrt{\lambda_{n}} f_{n}(\boldsymbol{x}) \xi_{n}(\theta),
$$

where $\bar{\alpha}(\boldsymbol{x})$ is the expected value of the random process, $\left\{\xi_{n}\right\}$ is a set of uncorrelated (not necessarily independent) random variables, $\left\{\lambda_{n}\right\}$ and $\left\{f_{n}\right\}$ are the eigenvalues and eigenfunctions of the covariance function $C_{\alpha \alpha}(\boldsymbol{x}, \boldsymbol{y})$ obtained by solving the following integral equation [1]

$$
\int_{\mathscr{D}} C_{\alpha \alpha}(\boldsymbol{x}, \boldsymbol{y}) f_{n}(\boldsymbol{y}) d \boldsymbol{y}=\lambda_{n} f_{n}(\boldsymbol{x}) .
$$

For example, consider an exponential covariance function of a stochastic process defined 
over a square domain $\mathscr{D}(x, y)$ over the interval $\left[\begin{array}{ll}-a & a\end{array}\right] \times\left[\begin{array}{ll}-a & a\end{array}\right][1]$

$$
C\left(x_{1}, y_{1} ; x_{2}, y_{2}\right)=\sigma^{2} e^{-\left|x_{2}-x_{1}\right| / b_{x}-\left|y_{2}-y_{1}\right| / b_{y}},
$$

using $b_{x}=b_{y}=b=1$, the correlation lengths along $x$ and $y$ directions respectively and $\sigma^{2}$ denotes the variance of the stochastic process.

Solving the integral equation given in Eq. (A.2) for the covariance kernel in Eq. (A.3), the eigenvalues and eigenfunctions are obtained as [1],

$$
\begin{gathered}
\lambda_{n}=\lambda_{i}^{x} \otimes \lambda_{i}^{y}, \\
f_{n}(x, y)=g_{i}(x) \otimes h_{i}(y) .
\end{gathered}
$$

where $\otimes$ denotes the tensor product (for example, see Table A.1), and

$$
\lambda_{i}^{x}=\lambda_{i}^{y}=\sigma \frac{2 b}{1+b^{2} \omega_{i}^{2}},
$$

and

$$
g_{i}(\boldsymbol{z})=h_{i}(\boldsymbol{z})= \begin{cases}\frac{\cos \left(\omega_{i} z\right)}{\sqrt{a+\frac{\sin \left(2 \omega_{i} a\right)}{2 \omega_{i}}}}, & \text { for } i \text { odd } \\ \frac{\sin \left(\omega_{i} z\right)}{\sqrt{a-\frac{\sin \left(2 \omega_{i} a\right)}{2 \omega_{i}}}}, & \text { for } i \text { even. }\end{cases}
$$

Here $\omega_{i}$ 's are the solution of the following transcendental equations [1,182],

$$
\begin{aligned}
& \frac{1}{b}-\omega_{i} \tan \left(\omega_{i} a\right)=0, \text { for } i \text { odd } \\
& \omega_{i}+\frac{1}{b} \tan \left(\omega_{i} a\right)=0, \text { for } i \text { even. }
\end{aligned}
$$

For simplicity, we refer $\left\{\lambda_{i}^{x}, \lambda_{i}^{y}\right\}$ and $\left\{g_{i}(x), h_{i}(y)\right\}$ are one-dimensional eigenvalues and eigenvectors. For example, tensor product notation in Eq. (A.4) for $\lambda_{i}^{x}=\left\{\lambda_{1}^{x}, \lambda_{2}^{x}\right\}$ and $\lambda_{i}^{y}=\left\{\lambda_{1}^{y}, \lambda_{2}^{y}\right\}$ leads to 


\begin{tabular}{|c|c|}
\hline two-dimensional eigenvalues & one-dimensional eigenvalues \\
\hline$\lambda_{1}$ & $\lambda_{1}^{x} \times \lambda_{1}^{y}$ \\
\hline$\lambda_{2}$ & $\lambda_{1}^{x} \times \lambda_{2}^{y}$ \\
\hline$\lambda_{3}$ & $\lambda_{2}^{x} \times \lambda_{1}^{y}$ \\
\hline$\lambda_{4}$ & $\lambda_{2}^{x} \times \lambda_{2}^{y}$ \\
\hline
\end{tabular}

Table A.1: Tensor product of one-dimensional eigenvalues.

Solving for one-dimensional $\omega_{i}$ and $\left\{\lambda_{i}^{x}, \lambda_{i}^{y}\right\}$ (which are always positive [224]) from Eq. (A.8) and Eq. (A.6) respectively for $b=1$ and $a=0.5$, i.e., using unit square domain, we get

\begin{tabular}{|l|c|c|c|c|c|c|c|}
\hline index $i$ & 1 & 2 & 3 & 4 & 5 & 6 & 7 \\
\hline$\omega_{i}$ & 1.306 & 3.673 & 6.585 & 12.723 & 15.834 & 18.955 & 22.082 \\
\hline$\lambda_{i}^{x}$ & 0.7388 & 0.1380 & 0.0451 & 0.0213 & 0.0123 & 0.0079 & 0.0056 \\
\hline
\end{tabular}

Table A.2: One-dimensional omegas $\omega_{i}$ and eigenvalues $\lambda_{i}^{x}, i=1,2, \ldots, 7$.

The results from eigenvalue analysis shown in Table A.2 are obtained by sorting eigenvalues $\left\{\lambda_{i}\right\}_{i=1}^{7}$ in descending order (i.e., largest to smallest eigenvalues). The first few eigenvalues account for most of the contribution to the variance and the contribution of higher indexed eigenvalues decreases quickly as shown in Fig. A.1. The two-dimensional eigenvalues are obtained by taking tensor product of (sorted) one-dimensional eigenvalues as shown in Table A.1. After taking the tensor product the two-dimensional eigenvalues are sorted in descending order are shown in Table A.3. This leads us to the new one-dimensional index in $x$ and $y$ dimensions, which we call sortIndex ${ }_{i}$ and sortIndex $_{j}$ as shown in Table A.3.

\begin{tabular}{|l|c|c|c|c|c|c|c|}
\hline index $n$ & 1 & 2 & 3 & 4 & 5 & 6 & 7 \\
\hline sortIndex $_{i}$ & $\lambda_{1}^{x}$ & $\lambda_{1}^{x}$ & $\lambda_{2}^{x}$ & $\lambda_{1}^{x}$ & $\lambda_{3}^{x}$ & $\lambda_{2}^{x}$ & $\lambda_{1}^{x}$ \\
\hline sortIndex $_{j}$ & $\lambda_{1}^{y}$ & $\lambda_{2}^{y}$ & $\lambda_{1}^{y}$ & $\lambda_{3}^{y}$ & $\lambda_{1}^{y}$ & $\lambda_{2}^{y}$ & $\lambda_{4}^{y}$ \\
\hline$\lambda_{n}$ & 0.5458 & 0.1020 & 0.1020 & 0.0333 & 0.0333 & 0.0190 & 0.0158 \\
\hline
\end{tabular}

Table A.3: Two-dimensional eigenvalues $\lambda_{n}, n=1,2, \ldots, 7$ and sortInde $_{i}=\{1,1,2,1,3,2,1\}$ and sortIndex $_{j}=\{1,2,1,3,1,2,4\}$.

From Fig. A.1 and A.2, it can be observed that the eigenvalue contribution decreases more rapidly in two-dimensional cases compared to one-dimensional cases. For example, to get the relative partial sum of eigenvalues $\left(\frac{\sum_{i}^{k} \lambda_{i}}{\sum_{i}^{n} \lambda_{i}}, n>>k\right)$ of $95 \%$, we need 20 eigenvalues in the twodimensional case (Eq. (A.4)) as oppose to only 4 modes in the one-dimensional case (Eq. (A.6)). 
Therefore, the number of random variables required to characterize the underlying stochastic process can increase with the physical dimension of the problem $[1,2,10]$.

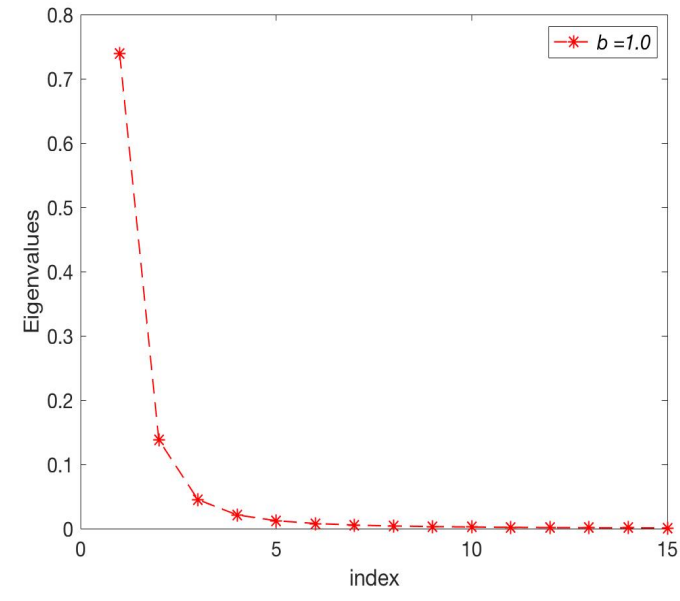

(a) one-dimensional eigenvalues (Eq. (A.6))

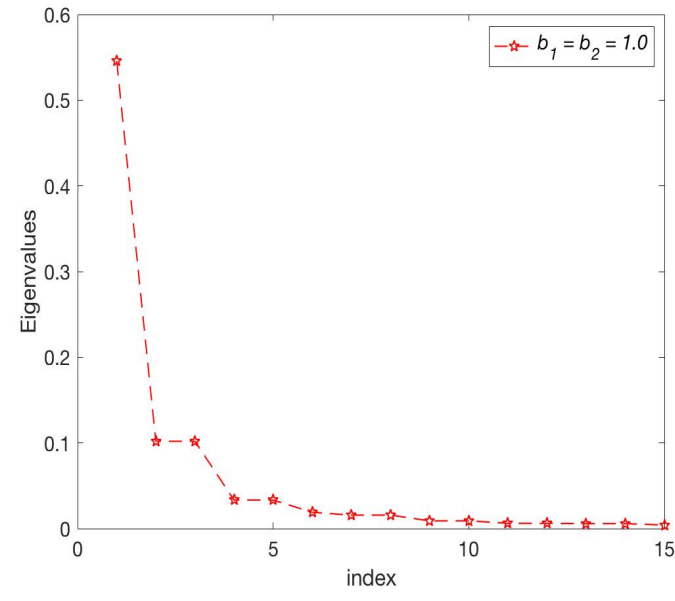

(b) two-dimensional eigenvalues (Eq. (A.4))

Figure A.1: Eigenvalues for $b=1.0$ and $a=0.5$

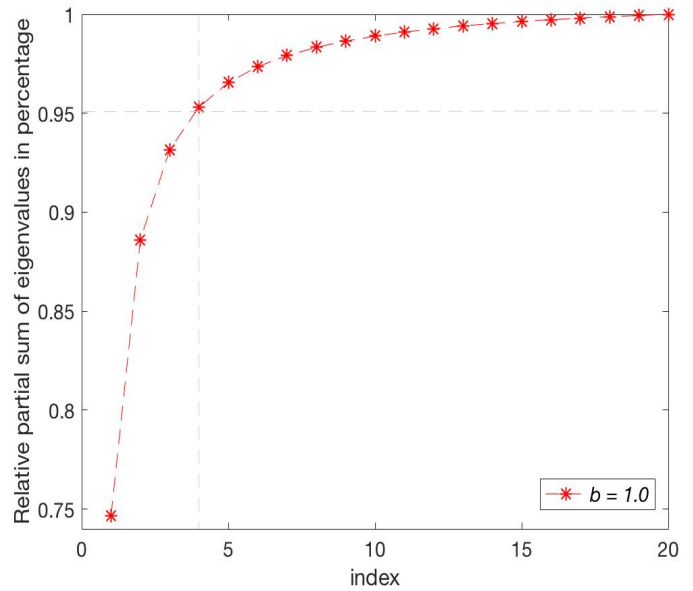

(a) one-dimensional case

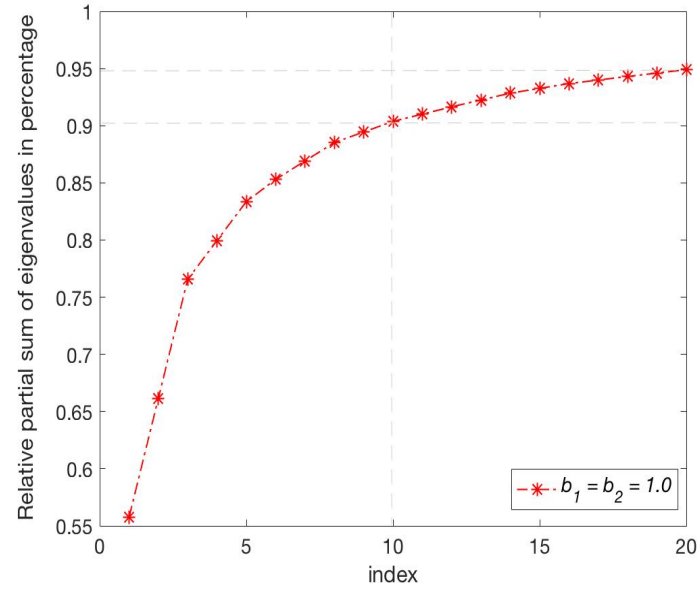

(b) two-dimensional case

Figure A.2: Relative partial sum of eigenvalues for $b=1.0$ and $a=0.5$ 


\section{A.2 Polynomial Chaos Expansion}

Consider a random process $\alpha(\boldsymbol{x}, \boldsymbol{\xi}(\theta))$, as function of position vector $\boldsymbol{x}$ and set of random variables $\boldsymbol{\xi}$ which are function of a random event $\theta$. Using polynomial chaos expansion (PCE) the stochastic process can be written as [182] (for notational convenience $\theta$ is dropped from henceforth),

$$
\begin{aligned}
\alpha(\boldsymbol{x}, \boldsymbol{\xi}) & =\widehat{\alpha}_{0} \Phi_{0}+\sum_{i_{1}=1}^{\infty} \widehat{\alpha}_{i_{1}} \Phi_{1}\left(\xi_{i_{1}}\right) \\
& +\sum_{i_{1}=1}^{\infty} \sum_{i_{2}=1}^{i_{1}} \widehat{\alpha}_{i_{1} i_{2}} \Phi_{2}\left(\xi_{i_{1}}, \xi_{i_{2}}\right) \\
& +\sum_{i_{1}=1}^{\infty} \sum_{i_{2}=1}^{i_{1}} \sum_{i_{3}=1}^{i_{2}} \widehat{\alpha}_{i_{1} i_{2} i_{3}} \Phi_{3}\left(\xi_{i_{1}}, \xi_{i_{2}}, \xi_{i_{3}}\right)+\ldots
\end{aligned}
$$

where the $\Phi_{n}\left(\xi_{i_{1}}, \ldots, \xi_{i_{n}}\right)$ are the multidimensional polynomial basis or polynomial chaoses [182] of order $n$ in terms of $n$-dimensional random variables $\left(\xi_{i_{1}}, \ldots, \xi_{i_{n}}\right)$. In Eq. (A.9), $\{\widehat{\alpha}\}$ are the deterministic PC coefficients which are function of $\boldsymbol{x}$.

For numerical implementation, a concise and truncated PC expansion is used. For instance, using $P_{u}$ terms, the PCE of $\alpha(\boldsymbol{x}, \boldsymbol{\xi})$ can be written as [182],

$$
\alpha(\boldsymbol{x}, \boldsymbol{\xi}) \approx \sum_{j=0}^{P_{u}} \alpha_{j}(\boldsymbol{x}) \Psi_{j}(\boldsymbol{\xi}) .
$$

There is an one-to-one relationship between the $\left\{\Phi_{i}\right\}$ and $\left\{\Psi_{j}\right\}$ and also $\left\{\widehat{\alpha}_{i}\right\}$ and $\left\{\alpha_{j}\right\}$ in Eq. (A.9) and Eq. (A.10). Note that, in this study, $\boldsymbol{\xi}$ is a Gaussian random vector and $\left\{\Psi_{j}\right\}$ are the Hermite polynomials. However, alternative representations using different types of random variables and polynomials are also available using generalized PCE [225]. 
The multidimensional polynomial chaoses up to the second order are given as [182]

$$
\begin{aligned}
\Psi_{0}(\xi) & =1, \\
\Psi_{1}\left(\xi_{i_{1}}\right) & =\xi_{i_{1}}, \\
\Psi_{2}\left(\xi_{i_{1}}, \xi_{i_{2}}\right) & =\xi_{i_{1}} \xi_{i_{2}}-\delta_{i_{1} i_{2}},
\end{aligned}
$$

where $\delta_{i_{1} i_{2}}$ denotes the Kronecker delta function defined as

$$
\delta_{i_{1} i_{2}}=\left\{\begin{array}{ccc}
1 & \text { if } & i_{1}=i_{2} \\
0 & \text { if } & i_{1} \neq i_{2}
\end{array} .\right.
$$

Note that, $\left\{\Psi_{i}\right\}$ are orthogonal in the statistical sense, i.e., their inner product $\left\langle\Psi_{i_{1}} \Psi_{i_{2}}\right\rangle$ is zero for $i_{1} \neq i_{2}$ (refer to Eq. (2.11) for details). For example, the second order PCE of $\alpha(\boldsymbol{x}, \boldsymbol{\xi})$ with three random variables $\left\{\xi_{1}, \xi_{2}, \xi_{3}\right\}$ is expanded as [1],

$$
\begin{aligned}
\alpha(\boldsymbol{x}, \boldsymbol{\xi})= & \alpha_{0} \Psi_{0}+\alpha_{1} \Psi_{1}+\alpha_{2} \Psi_{2}+\alpha_{3} \Psi_{3}+\alpha_{4} \Psi_{4}+\alpha_{5} \Psi_{5} \\
& +\alpha_{6} \Psi_{6}+\alpha_{7} \Psi_{7}+\alpha_{8} \Psi_{8}+\alpha_{9} \Psi_{9} \\
= & \alpha_{0}+\alpha_{1} \xi_{1}+\alpha_{2} \xi_{2}+\alpha_{3} \xi_{3}+\alpha_{4}\left(\xi_{1}^{2}-1\right)+\alpha_{5} \xi_{1} \xi_{2} \\
& +\alpha_{6}\left(\xi_{2}^{2}-1\right)+\alpha_{7} \xi_{1} \xi_{3}+\alpha_{8}\left(\xi_{3}^{2}-1\right)+\alpha_{9} \xi_{2} \xi_{3} .
\end{aligned}
$$

The explicit expressions for the polynomials used in Eq. (A.13) are shown in Table A.4. The number of terms $P_{u}$ required in a PCE, with order $p$ and dimension $L$ can be obtained as [182],

$$
P_{u}=\frac{(L+p) !}{L ! p !}-1
$$

For the numerical implementation of PCE, one can generalized the evaluation of multidimensional polynomials $\left\{\Psi_{i}\right\}$ using one-dimensional polynomials $\left\{\psi_{i}\right\}$ and multi-index $m_{i}^{j}$ defined in [88]. This approach is quite useful in the automation of PCE basis function evaluation 


\begin{tabular}{|c|c|c|c|}
\hline$j^{\text {th }}$ PC term & Order of the expansion & $\Psi_{j}$ & $\left\langle\Psi_{j}^{2}\right\rangle$ \\
\hline 0 & 0 & 1 & 1 \\
\hline 1 & 1 & $\xi_{1}$ & 1 \\
2 & & $\xi_{1}$ & 1 \\
3 & 2 & $\xi_{3}$ & 1 \\
\hline 4 & & $\xi_{1}^{2}-1$ & 2 \\
5 & & $\xi_{1} \xi_{2}$ & 1 \\
6 & & $\xi_{2}^{2}-1$ & 2 \\
7 & & $\xi_{1} \xi_{3}$ & 1 \\
8 & & $\xi_{3}^{2}-1$ & 2 \\
9 & & $\xi_{2} \xi_{3}$ & 1 \\
\hline
\end{tabular}

Table A.4: Polynomial chaoses and their variances for a second-order and three-dimensional PCE [1]

and calculation of their moments for high-dimensional PC expansions used in this thesis. For demonstration, consider evaluations of the $L$-dimensional polynomial chaoses and their moments using one-dimensional polynomials. The form of the one-dimensional Hermite polynomials are given below.

$$
\begin{aligned}
& \psi_{0}=1, \\
& \psi_{1}=\xi \\
& \psi_{2}=\xi^{2}-1 \\
& \psi_{n}=\xi \psi_{n-1}-(n-1) \psi_{n-2 .} .
\end{aligned}
$$

The $L$-dimensional polynomial chaoses can be obtained from [9]:

$$
\Psi_{j}\left(\xi_{1}, \xi_{2}, \ldots, \xi_{L}\right)=\prod_{i=1}^{L} \psi_{m_{i}^{j}}\left(\xi_{i}\right)
$$

where $m_{i}^{j}$ denotes multi-index. The code adapted from UQTk [29] is employed in this thesis to get the multi-index (refer to [9] for further details on multi-index definition and construction). A snippet of the Matlab code used for the evaluation of multidimensional Hermite polynomials in Eq. (A.17) is given in Listing A.1. Similar procedure can be employed to evaluate the moments 
of multidimensional polynomials $\left\{\Psi_{i}\right\}$ using moments of one-dimensional polynomials and multi-index [9]. The moment of multidimensional polynomials $\left\{\Psi_{i}\right\}$ of order $p$ and dimension $L$ can be obtained using one-dimensional polynomials $\left\{\psi_{i}\right\}$ and multi-index $m_{i}^{j}$ as:

$$
\left\langle\prod_{n=1}^{p} \Psi_{j_{n}}\right\rangle_{L}=\prod_{i=1}^{L}\left\langle\prod_{n=1}^{p} \psi_{m_{i}^{j n}}\right\rangle_{1}
$$

In this thesis, the code adapted from UQTk [29] is employed to evaluate moments of multidimensional polynomials. The Matlab code snippet to evaluate the moments of $L$-dimensional Hermite polynomials using moments of one-dimensional polynomials is given in Listing B.6 in Appendix B. Direct evaluation of moments of multidimensional polynomials by solving multidimensional integral is computationally expensive, especially for the high-dimensional cases considered in this thesis.

Listing A.1: L-dimensional polynomials using 1-dimensional polynomials

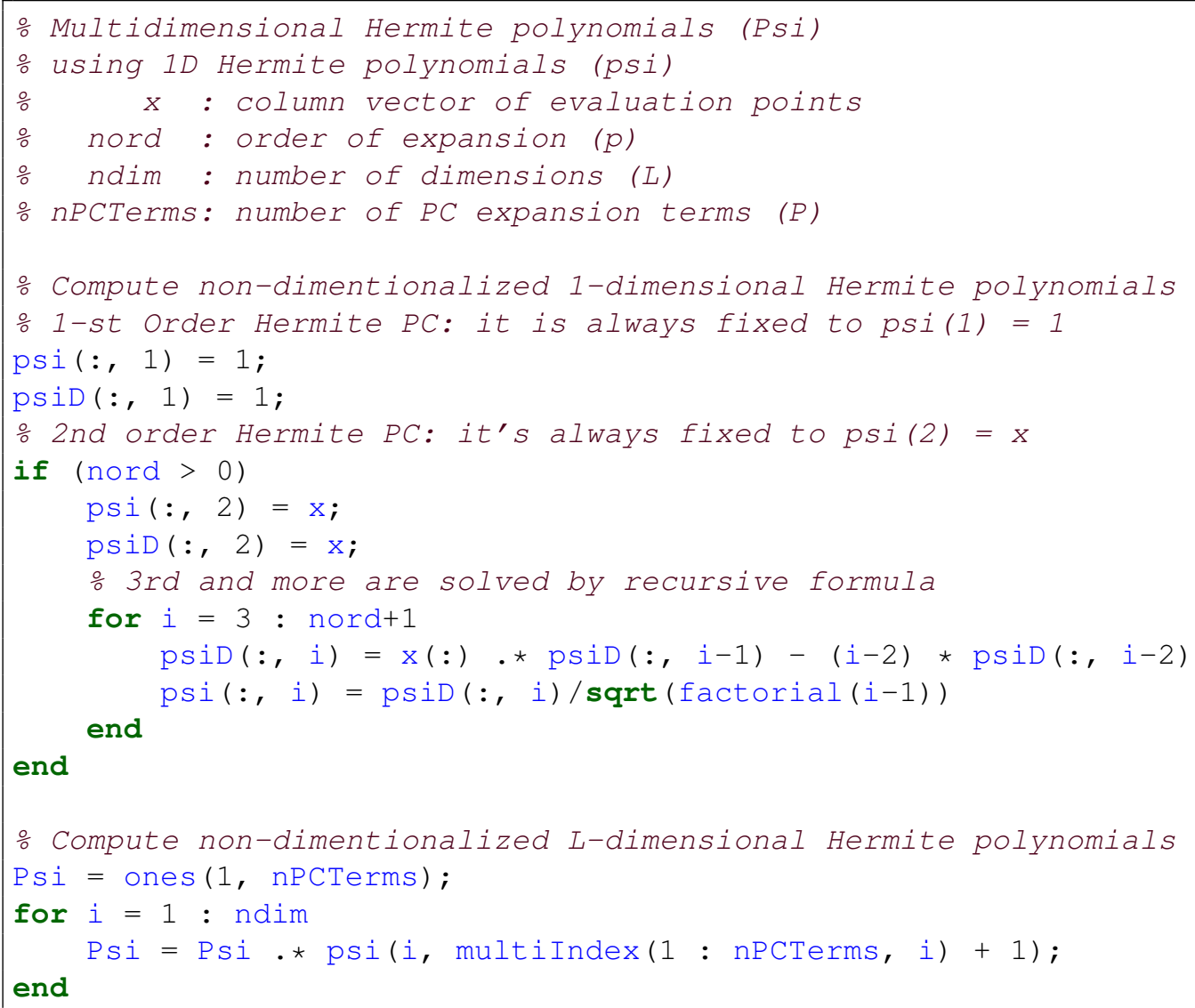




\section{A.3 Spectral Representation of Lognormal Stochastic}

\section{Process using PCE}

The PCE of a lognormal stochastic process $l(\boldsymbol{x}, \theta)$, obtained by exponential of a Gaussian process $g(\boldsymbol{x}, \theta)$, with a covariance function $C_{\alpha \alpha}$ and variance $\sigma^{2}$ defined over a given domain, for instance, as shown in Eq. (A.3),

$$
l(\boldsymbol{x}, \theta)=\exp [g(\boldsymbol{x}, \theta)]
$$

The underlying Gaussian process $g(\boldsymbol{x}, \theta)$ is characterized by using a truncated KLE with $L$ random variables as follows,

$$
g(\boldsymbol{x}, \theta)=g_{0}(\boldsymbol{x})+\sum_{j=1}^{L} g_{j}(\boldsymbol{x}) \xi_{j}(\theta)
$$

where $g_{0}(\boldsymbol{x})$ is the mean and $g_{j}(\boldsymbol{x})=\sqrt{\lambda_{j}} f_{j}(\boldsymbol{x})$ with $\left\{\lambda_{j}\right\}$ and $\left\{f_{j}\right\}$ denoting eigenvalues and eigenvectors respectively as defined in Appendix A.1. The lognormal stochastic process in Eq. (A.19) can be rewritten using Eq. (A.20) as

$$
l(\boldsymbol{x}, \theta)=\exp \left[g_{0}(\boldsymbol{x})+\sum_{j=1}^{L} g_{j}(\boldsymbol{x}) \xi_{j}(\theta)\right]
$$

The lognormal process $l(\boldsymbol{x}, \theta)$ can be expanded using PCE as follows,

$$
l(\boldsymbol{x}, \theta)=\sum_{i=0}^{P_{\alpha}} l_{i}(\boldsymbol{x}) \Psi_{i}(\boldsymbol{\xi})
$$

where $P_{\alpha}$ is number of PCE terms obtained by using Eq. (A.15) and $\left\{l_{i}(\boldsymbol{x})\right\}_{i=0}^{P_{\alpha}}$ are the PCE coefficients of the lognormal process $l(\boldsymbol{x}, \theta)$. 
Performing Galerkin projection, $l_{i}(\boldsymbol{x})$ can be obtained as [1,2]

$$
l_{i}(\boldsymbol{x})=\frac{\left\langle l(\boldsymbol{x}, \theta) \Psi_{i}(\boldsymbol{\xi})\right\rangle}{\left\langle\Psi_{i}^{2}(\boldsymbol{\xi})\right\rangle}
$$

The denominator in Eq. (A.23) can be evaluated analytically beforehand, for instance see Table A.4. The numerator in Eq. (A.23) can be expressed as an integral $[2,183]$

$$
\left\langle l(\boldsymbol{x}, \theta) \Psi_{i}(\boldsymbol{\xi})\right\rangle=\int_{-\infty}^{+\infty} \exp \left[g_{0}(\boldsymbol{x})+\sum_{j=1}^{L} g_{j}(\boldsymbol{x}) \xi_{j}\right] \Psi_{i}(\boldsymbol{\xi}) \exp \left[-\frac{1}{2} \sum_{j=1}^{L} \xi_{j}^{2}\right] d \boldsymbol{\xi}
$$

Eq. (A.24) can be simplified to [24]

$$
\left\langle l(\boldsymbol{x}, \theta) \Psi_{i}(\boldsymbol{\xi})\right\rangle=\exp \left[g_{0}(\boldsymbol{x})+\frac{1}{2} \sum_{j=1}^{L} g_{j}^{2}(\boldsymbol{x})\right]\left\langle\Psi_{j}(\boldsymbol{\eta})\right\rangle
$$

where $\boldsymbol{\eta}_{j}=\boldsymbol{\xi}_{j}-g_{j}(\boldsymbol{x})$. Eq. (A.25) can be rewritten in more concise form as

$$
\left\langle l(\boldsymbol{x}, \theta), \Psi_{i}(\boldsymbol{\xi})\right\rangle=l_{0}(\boldsymbol{x})\left\langle\Psi_{j}(\boldsymbol{\eta})\right\rangle
$$

where $l_{0}(\boldsymbol{x})$ represents the mean of the lognormal process $l(\boldsymbol{x}, \theta)$

$$
l_{0}(\boldsymbol{x})=\exp \left[g_{0}(\boldsymbol{x})+\frac{1}{2} \sum_{j=1}^{L} g_{j}^{2}(\boldsymbol{x})\right]
$$

As the number of KLE terms, $L$ tends to $\infty$, the mean of $l(\boldsymbol{x}, \theta)$ converges to [2]

$$
l_{0}(\boldsymbol{x})=\exp \left[g_{0}(\boldsymbol{x})+\frac{1}{2} \sigma^{2}\right] .
$$

Using Eq. (A.23) and Eq. (A.25), the PCE of the lognormal stochastic process $l(\boldsymbol{x}, \theta)$ can be written as

$$
l(\boldsymbol{x}, \theta)=l_{0}(\boldsymbol{x}) \sum_{i=0}^{P_{\alpha}} \frac{\left\langle\Psi_{i}(\boldsymbol{\eta})\right\rangle}{\left\langle\Psi_{i}^{2}(\boldsymbol{\xi})\right\rangle} \Psi_{i}(\boldsymbol{\xi}) .
$$




\begin{tabular}{|c|c|c|}
\hline$\Psi_{i}(\boldsymbol{\xi})$ & $\left\langle\Psi_{i}^{2}(\boldsymbol{\xi})\right\rangle$ & $\left\langle\Psi_{j}(\boldsymbol{\eta})\right\rangle$ \\
\hline$\xi_{1}$ & 1 & $g_{1}(\boldsymbol{x})$ \\
$\xi_{1}$ & 1 & $g_{2}(\boldsymbol{x})$ \\
$\xi_{3}$ & 1 & $g_{i}(\boldsymbol{x})$ \\
\hline$\xi_{1}^{2}-1$ & 2 & $g_{1}^{2}(\boldsymbol{x})$ \\
$\xi_{1} \xi_{2}$ & 1 & $g_{1}(\boldsymbol{x}) g_{2}(\boldsymbol{x})$ \\
$\xi_{2}^{2}-1$ & 2 & $g_{2}^{2}(\boldsymbol{x})$ \\
$\xi_{1} \xi_{3}$ & 1 & $g_{1}(\boldsymbol{x}) g_{3}(\boldsymbol{x})$ \\
$\xi_{3}^{2}-1$ & 2 & $g_{3}^{2}(\boldsymbol{x})$ \\
$\xi_{2} \xi_{3}$ & 1 & $g_{2}(\boldsymbol{x}) g_{3}(\boldsymbol{x})$ \\
\hline
\end{tabular}

Table A.5: The expectation and variance of second-order and three-dimensional PC basis [2].

The $\left\langle\Psi_{j}(\boldsymbol{\eta})\right\rangle$ represents the expectation of the PC basis around the coefficients $g_{i}(\boldsymbol{x})$. These expectations can be evaluated analytically. For instance, see Table A.5 showing variance $\left\langle\Psi_{i}^{2}(\boldsymbol{\xi})\right\rangle$ and expectations $\left\langle\Psi_{i}(\boldsymbol{\eta})\right\rangle$ for the second-order and three-dimensional PC basis functions. For further simplification, using $L=3$ and the second order ( $p=2$ ) expansion leading to the number of PCE terms $P_{\alpha}=9$, Eq. (A.29) can be expand as (note $\boldsymbol{x}$ of $g_{i}$ is dropped for notational convenience),

$$
\begin{aligned}
l(\boldsymbol{x}, \theta) & =l_{0}\left(1+\xi_{1} g_{1}+\xi_{2} g_{2}+\xi_{3} g_{3}+\left(\xi_{1}^{2}-1\right) \frac{g_{1}^{2}}{2}\right. \\
& \left.+\left(\xi_{1} \xi_{2}\right) g_{1} g_{2}+\left(\xi_{2}^{2}-1\right) \frac{g_{2}^{2}}{2}+\left(\xi_{1} \xi_{3}\right) g_{1} g_{3}+\left(\xi_{3}^{2}-1\right) \frac{g_{3}^{2}}{2}+\left(\xi_{2} \xi_{3}\right) g_{2} g_{3}\right)
\end{aligned}
$$

For illustration, consider a simplest case where $g(\boldsymbol{x}, \theta)$ is characterized by using a Gaussian random variable $\xi$ with the mean $\mu_{g}$ and variance $\sigma_{g}^{2}$. The lognormal random variable $l(\boldsymbol{x}, \theta)$ can be obtained using procedure outlined above [2],

$$
\begin{aligned}
l(\boldsymbol{x}, \theta) & =\mu_{l} \sum_{i=0}^{L} \frac{\sigma_{g}^{i}}{i !} \Psi_{i}(\xi) \\
& =\mu_{l}\left(1+\frac{\sigma_{g}}{1 !} \xi+\frac{\sigma_{g}^{2}}{2 !}\left(\xi^{2}-1\right)+\ldots\right)
\end{aligned}
$$

where $\mu_{l}=\exp \left[\mu_{g}+\frac{1}{2} \sigma_{g}^{2}\right]$ is the mean of the lognormal random variable. 


\section{Appendix B}

\section{Spectral Stochastic Finite Element Method Formulation, Algorithms and Codes}

The finite element discretization of stochastic PDE given in Eq. (2.14) leads to the following stochastic system of linear equation $[1,32,73]$ :

$$
\mathbf{A}(\theta) \mathbf{u}(\theta)=\mathbf{f}
$$

The above system can be solved for the mean or any sample of the stochastic parameter $c_{d}(\boldsymbol{x}, \theta)$ by using any deterministic FEM solver. For demonstration we employed FEniCS general purpose deterministic FEM packages [32]. The corresponding FEniCS based python code snippet is shown in Lisitng B.1.

Listing B.1: FEniCS based python code snippet for deterministic FEM

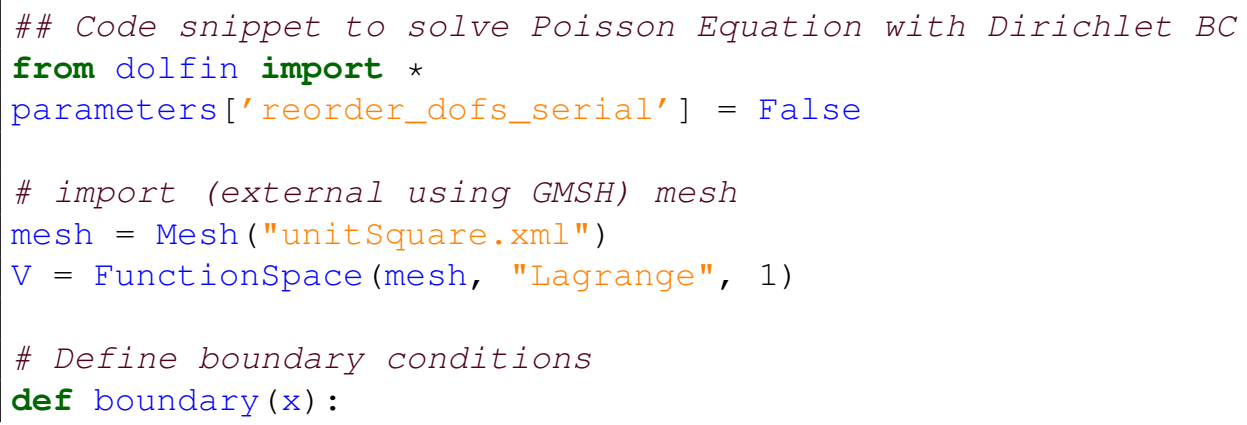




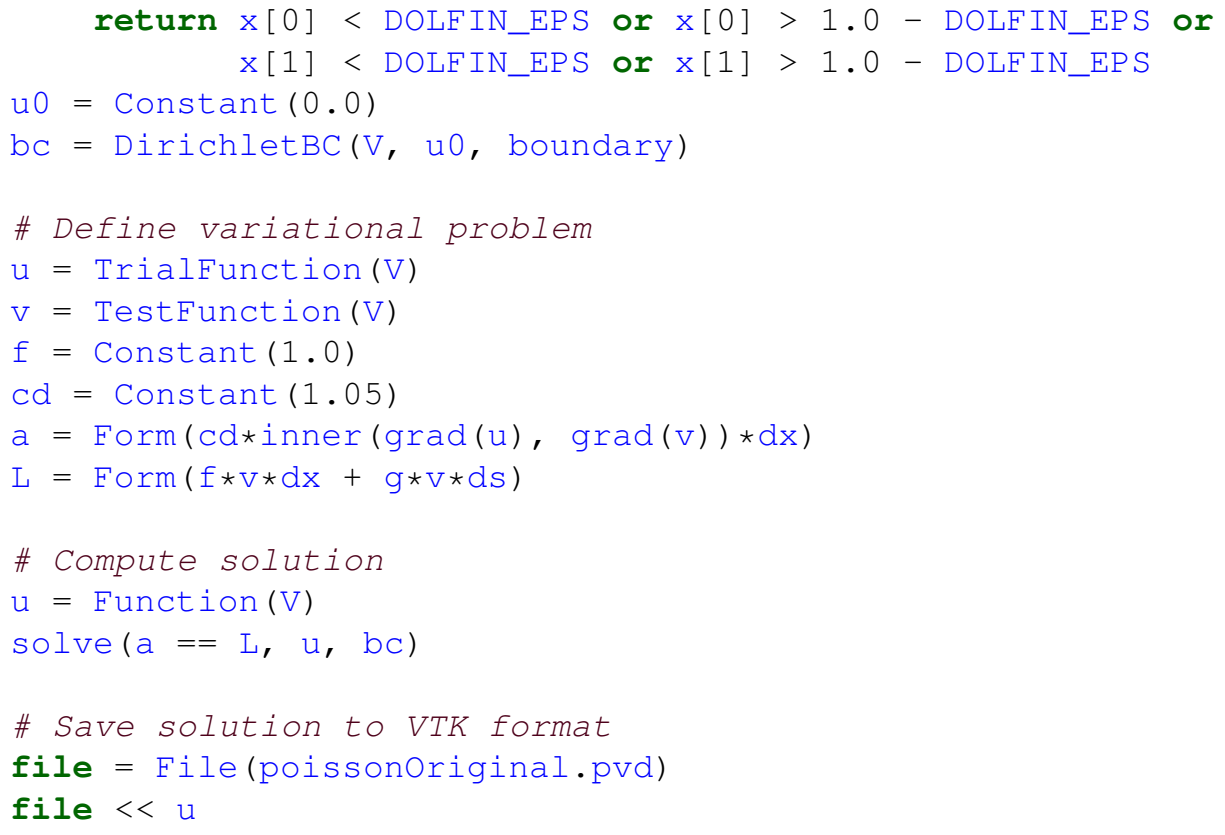

\section{B.1 Spectral Stochastic Finite Element Methods}

The stochastic system matrix $\mathbf{A}(\theta)$ and the stochastic solution process $\mathbf{u}(\theta)$ in Eq. (B.1) are approximated using the polynomial chaos expansions as [1],

$$
\mathbf{A}(\theta) \approx \sum_{i=0}^{P_{\mathrm{A}}} \hat{\mathbf{A}}_{i} \Psi_{i}(\boldsymbol{\xi}), \quad \mathbf{u}(\theta) \approx \sum_{j=0}^{P_{u}} \hat{\mathbf{u}}_{j} \Psi_{j}(\boldsymbol{\xi})
$$

where $\hat{\mathbf{A}}_{i}$ 's are the PCE coefficients of the random system matrix, $\hat{\mathbf{u}}_{j}$ 's are the PCE coefficients of the solution process and $\Psi_{j}$ 's are the multidimensional polynomials obtained as a function of $L$ random variables $\boldsymbol{\xi}=\left\{\xi_{1}, \xi_{2}, \ldots, \xi_{L}\right\} . P_{\mathrm{A}}$ and $P_{u}$ are the numbers of PCE terms required to express the stochastic system matrix and the solution process, respectively [1].

The PCE coefficients ( $\hat{\mathbf{A}}_{i}$ 's) are computed using a lognormal diffusion coefficient obtained from a underlying Gaussian process expanded using $\operatorname{KLE}$ [1, 10, 90] (refer to the procedure outlined in Appendix A.1). Therefore, in the SSFEM approaches, the primary goal is to estimate the PCE coefficients $\left(\hat{\mathbf{u}}_{j}\right)$ of the solution process $\mathbf{u}(\theta)$. 


\section{B.2 Intrusive SSFEM}

In the intrusive SSFEM, the PCE of the input and output stochastic processes presented in Eq. (B.2) are directly substituted into the finite element discretization of stochastic PDE given in Eq. (B.1) leading to,

$$
\sum_{i=0}^{P_{\mathrm{A}}} \hat{\mathbf{A}}_{i} \Psi_{i}(\boldsymbol{\xi}) \sum_{j=0}^{P_{u}} \hat{\mathbf{u}}_{j} \Psi_{j}(\boldsymbol{\xi}) \approx \mathbf{f}
$$

Applying the procedure detailed in Sec. 2.2.1, Eq. (B.3) can be written as

$$
\left[\sum_{j=0}^{P_{u}} \sum_{i=0}^{P_{\mathrm{A}}} C_{i j k} \hat{\mathbf{A}}_{i}\right]\left\{\hat{\mathbf{u}}_{k}\right\}=\left\{f_{k}\right\}, \quad k=0, \ldots, P_{u} .
$$

where $C_{i j k}=\left\langle\Psi_{i}(\boldsymbol{\xi}) \Psi_{j}(\boldsymbol{\xi}) \Psi_{k}(\boldsymbol{\xi})\right\rangle$ and $f_{k}=\left\langle\mathbf{f}, \Psi_{k}(\boldsymbol{\xi})\right\rangle$.

The stochastic system matrix in Eq. (B.4) can be assembled using the procedure outline in Algorithm 12. This procedure employs deterministic, element-level, FEniCS-based assemble routines. It is designed to reduce the number of call to the deterministic assembly routine.

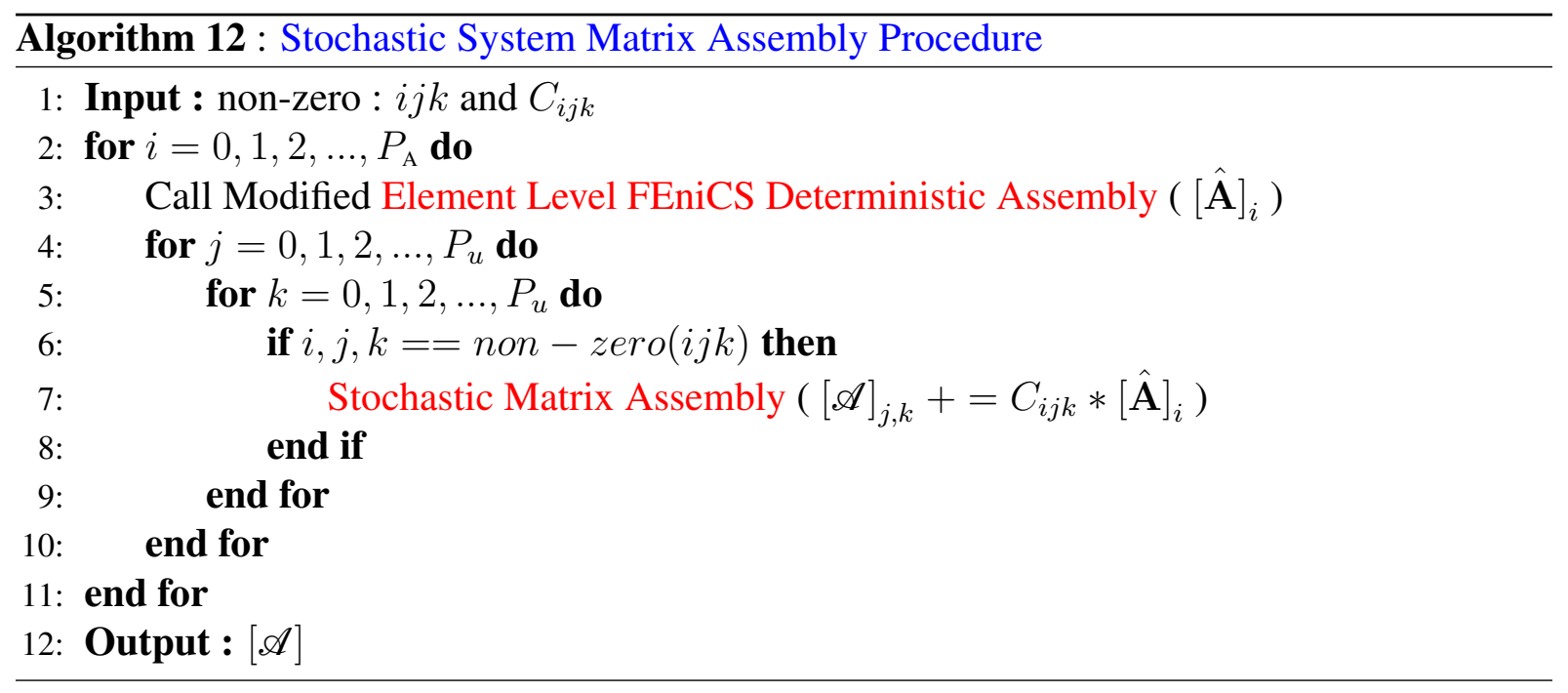

The code snippet to perform intrusive SSFEM system matrix assembly using FEniCS-based assembly routine is presented in Listing B.2. The procedure to call to assemble_local, an element-level FEniCS assembly routines [32] is outlined in Listing B.3. For each of the input PCE term, the procedure outlined in the Listing B.3 is invoked once. The FEniCS-based proce- 
dure to define stochastic variational form for steady state diffusion equation defined in Eq. (2.17) is outlined in Listing B.4. The diffusion coefficient $c_{d}$ is characterized as a lognormal stochastic process. The diffusion coefficient $c_{d}$ is defined as a FEniCS-based, expression-class [32], as outlined in Listing B.5.

The parameters such as $\omega, \lambda$, sort-index and multi-index required to perform the SSFEMsystem matrix assembly procedure, are need to be computed beforehand. The procedure outlined earlier in Appendix A.1 and Appendix A.2 can be used to calculate $\omega_{i}, \lambda_{i}$, and sort-index. The multi-index calculation is performed using functions from UQ ToolKit [29]. The procedure to calculate moments of the multidimensional polynomials $C_{i j k}$ and non-zero $i, j$ and $k$ indices, required for the Listing B.2 is outlined in the Listing B.6. This procedure is developed by adapting functions from UQ ToolKit [29].

Listing B.2: FEniCS based intrusive SSFEM assembly code snippet

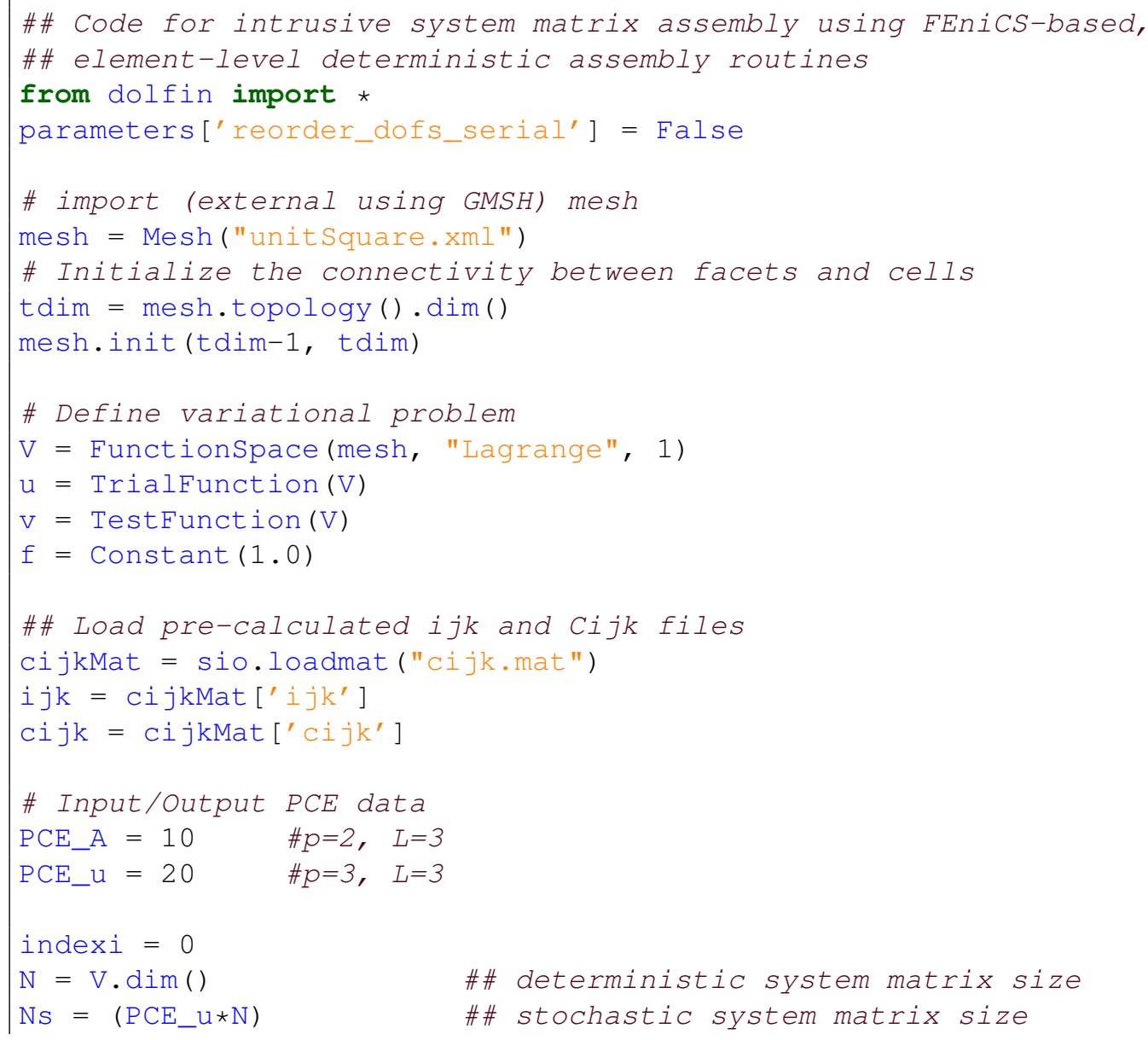




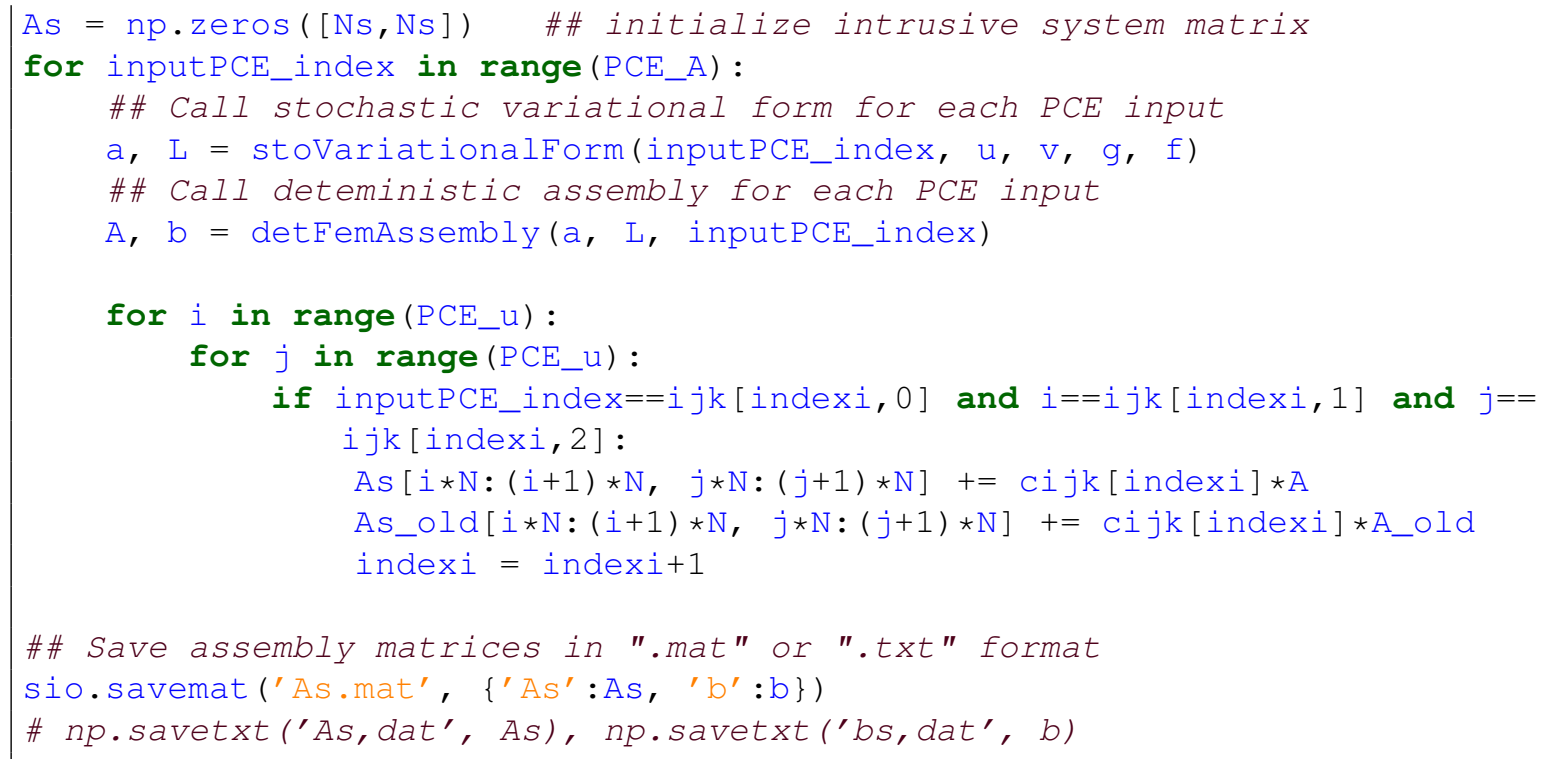

\section{Listing B.3: Python code snippet for FEniCS based (element-level) FEM assembly}

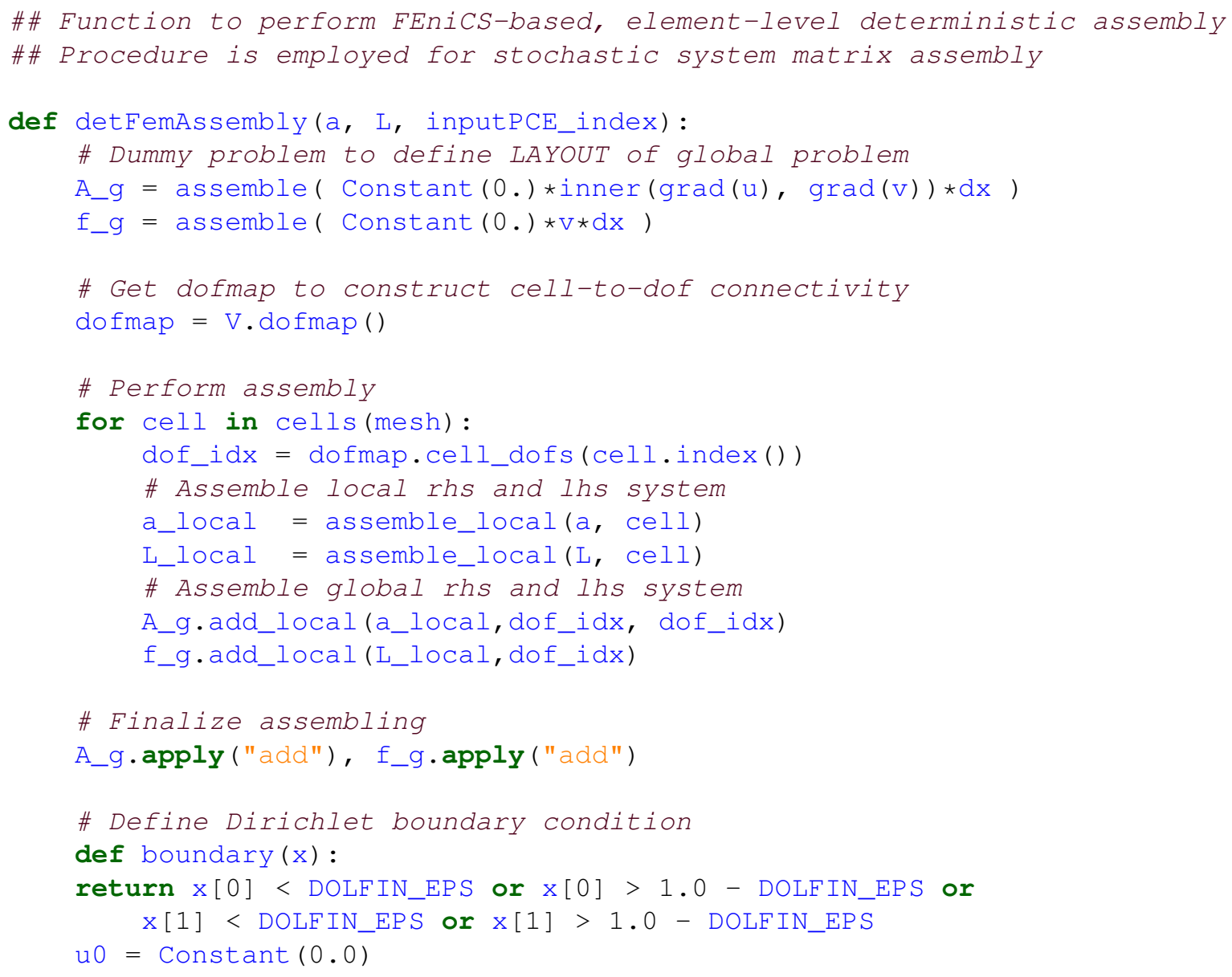




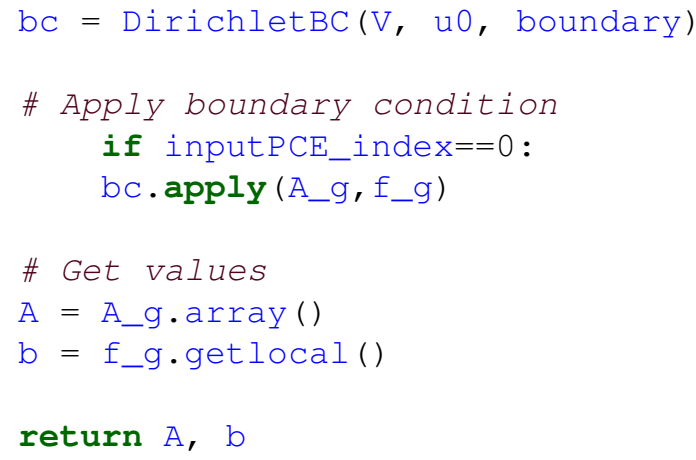

\section{Listing B.4: Python code snippet for FEniCS based stochastic variational form of Poisson equation}

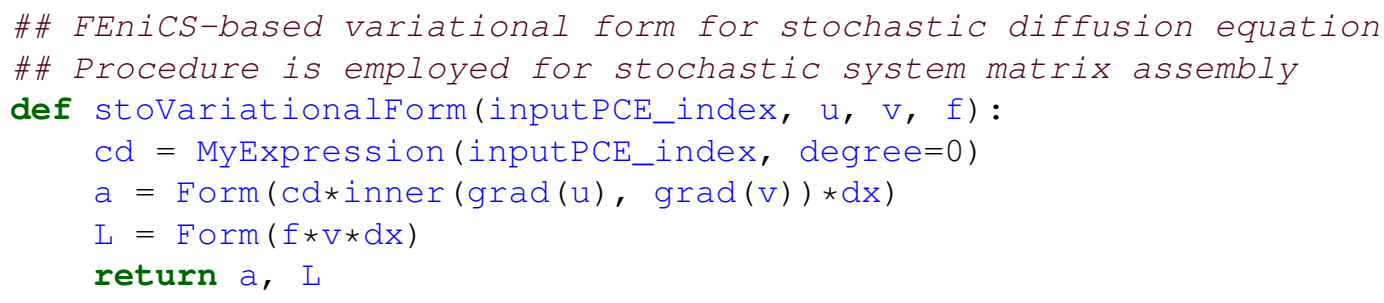

\section{Listing B.5: Python code snippet for PC expansion of lognormal stochastic process}

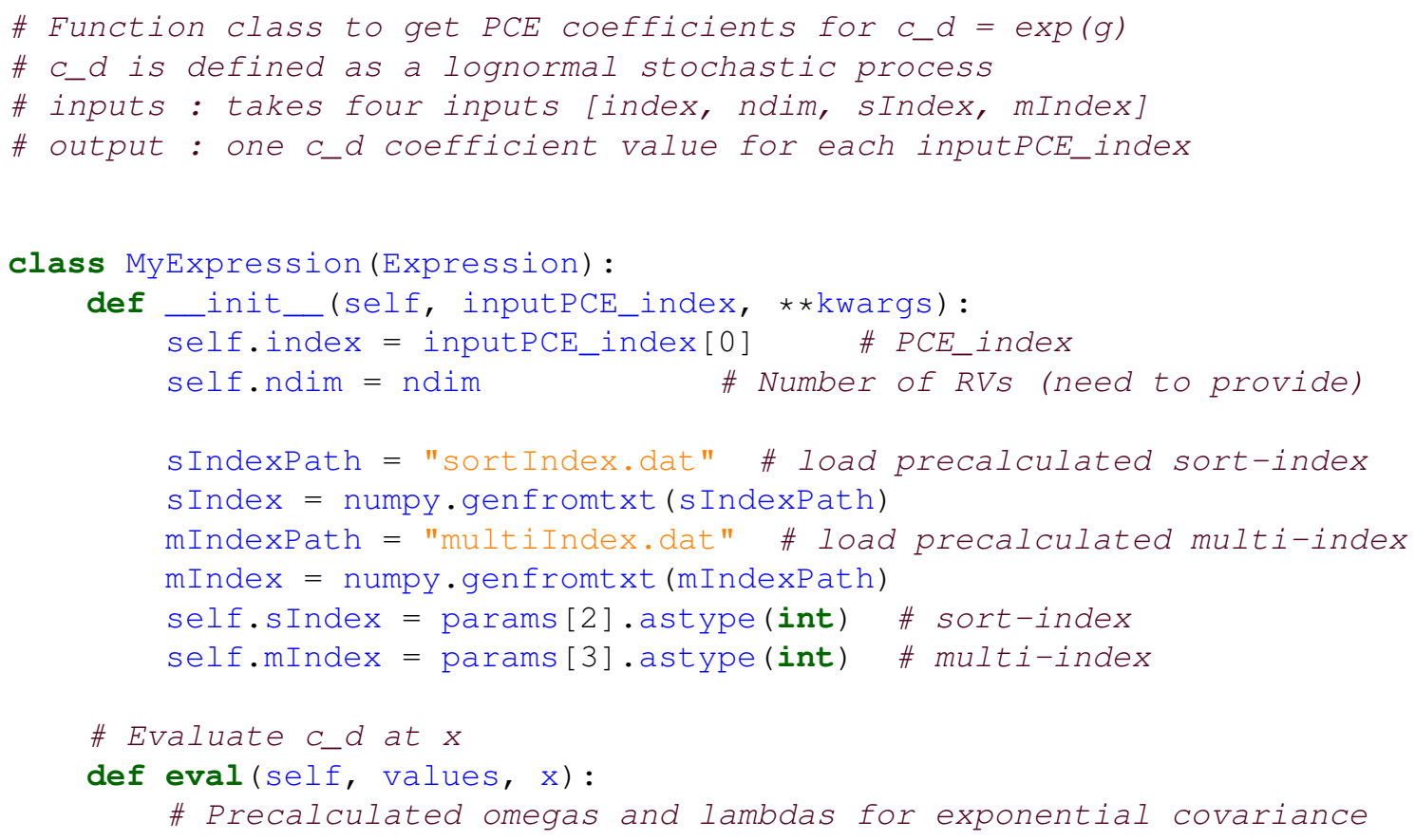




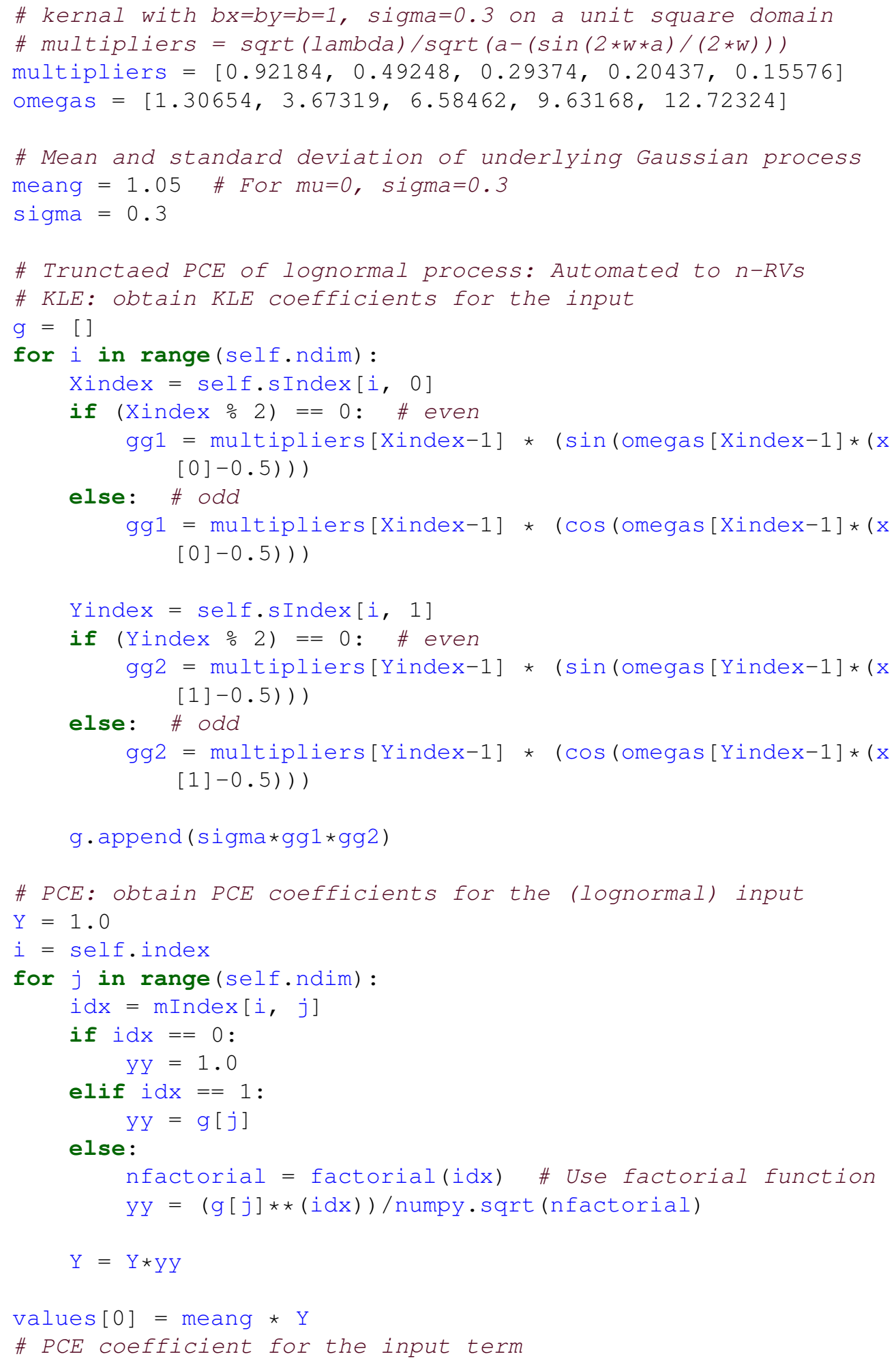




\section{Listing B.6: Matlab code to compute moments of ND-polynomials using 1D-polynomials}

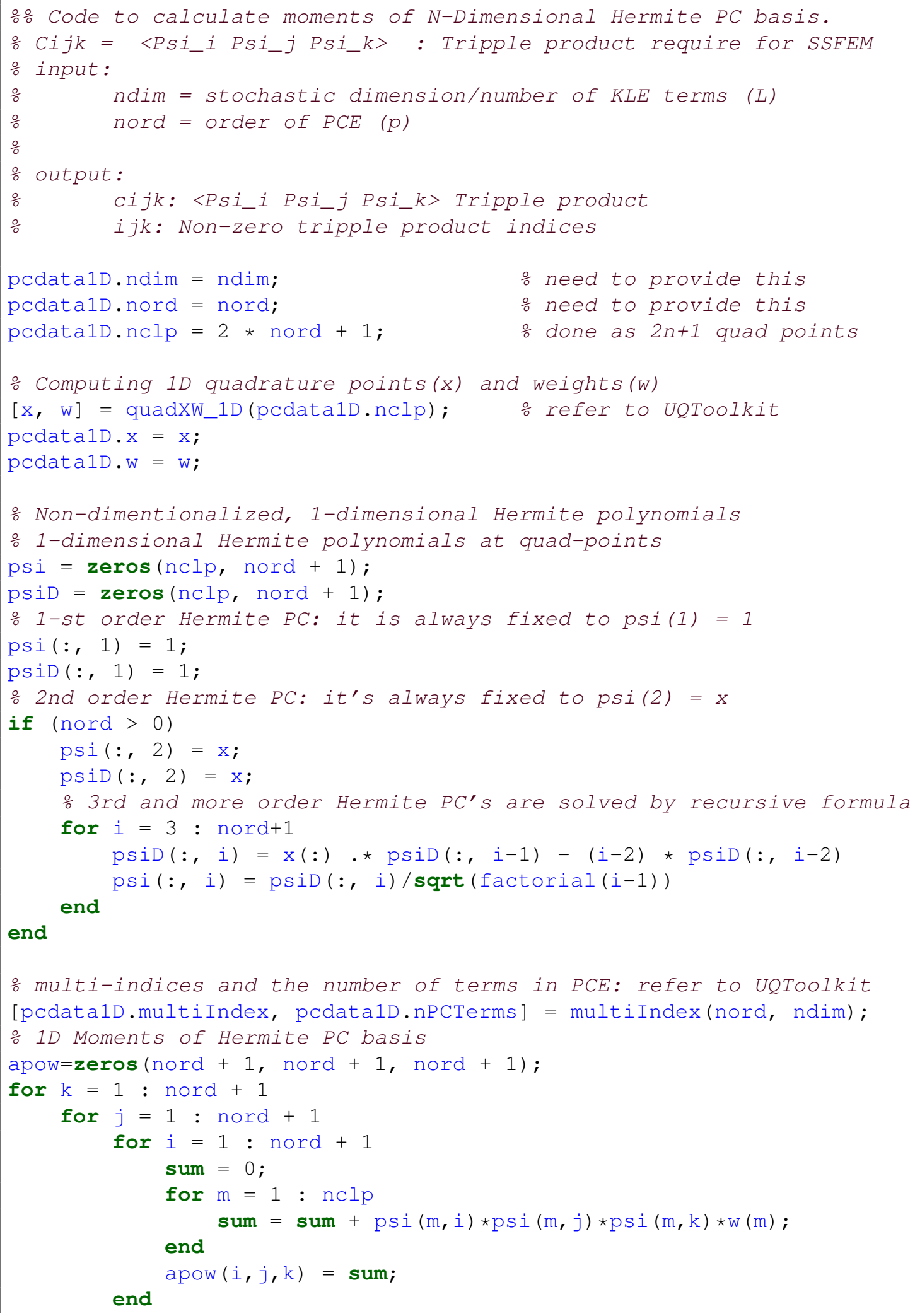




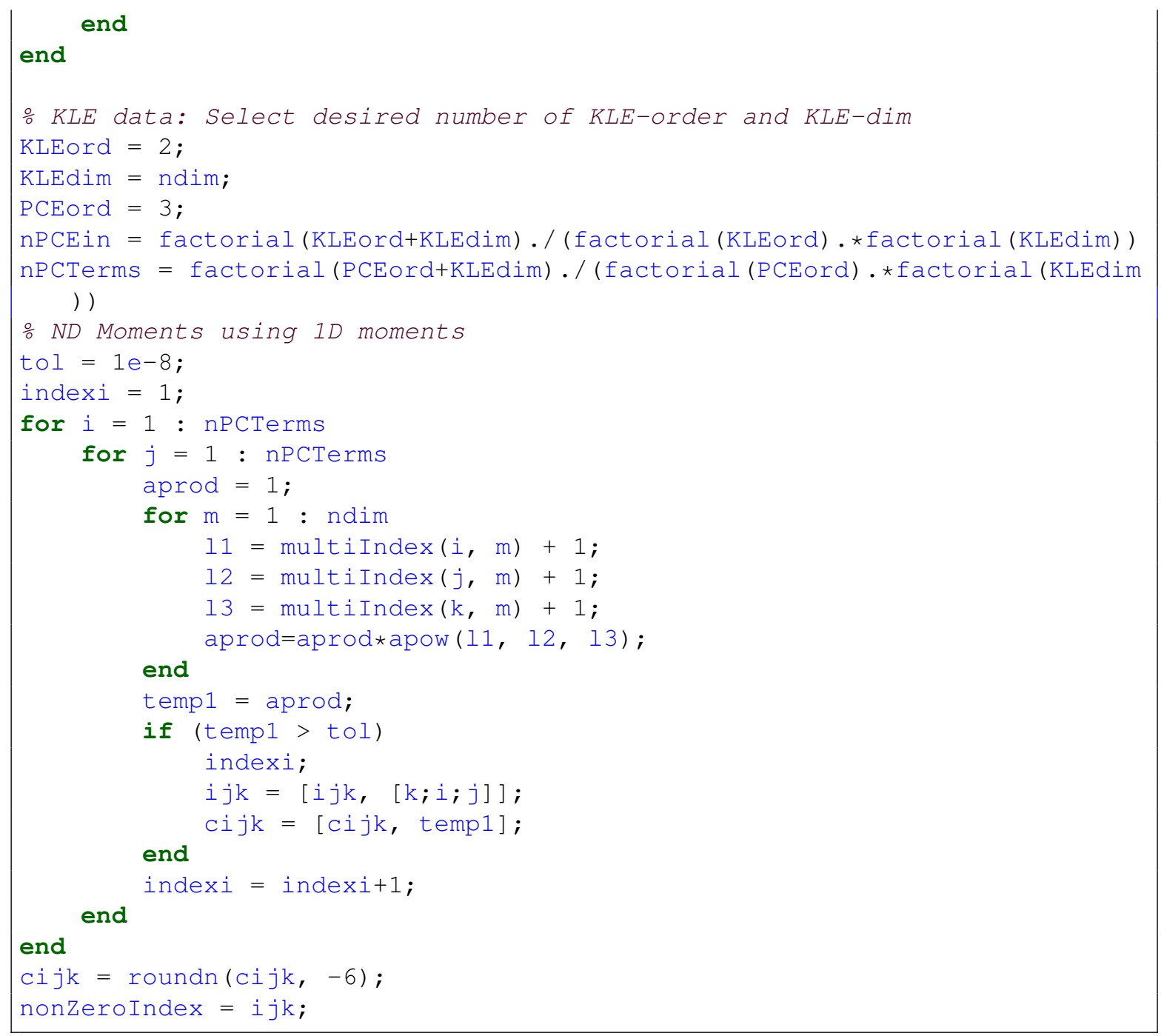

The numerical simulations are performed using FEniCS package for the stochastic PDE defined by Eq. (2.14), using the following numerical parameters $b=1, \sigma=0.3, L=3, p_{u}=3$ and $f=1$. A unit square domain, discretized using 600 nodes and 1200 elements is used. The mean and standard deviation of the solution field are plotted in Fig. B.1. A few selected PCE coefficients are plotted in Fig. B.2. 

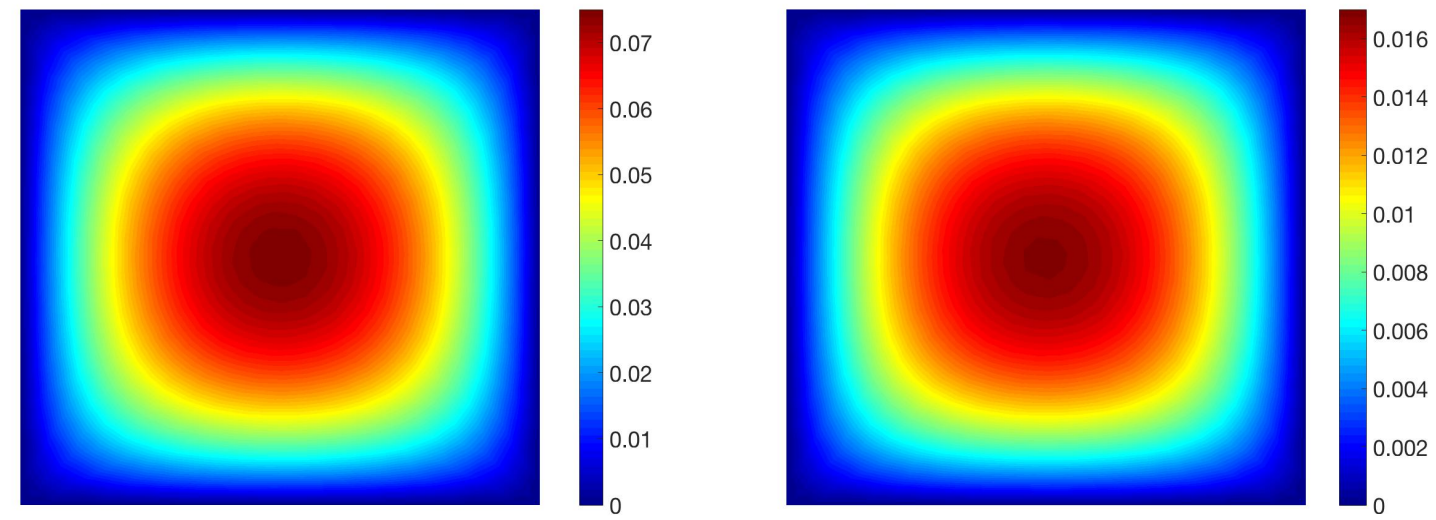

Figure B.1: Mean and standard deviation of the solution field.

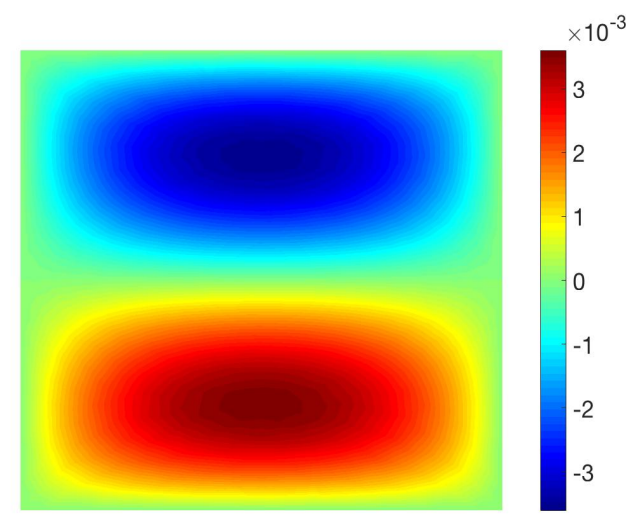

(a) $\hat{\mathbf{u}}_{4}$

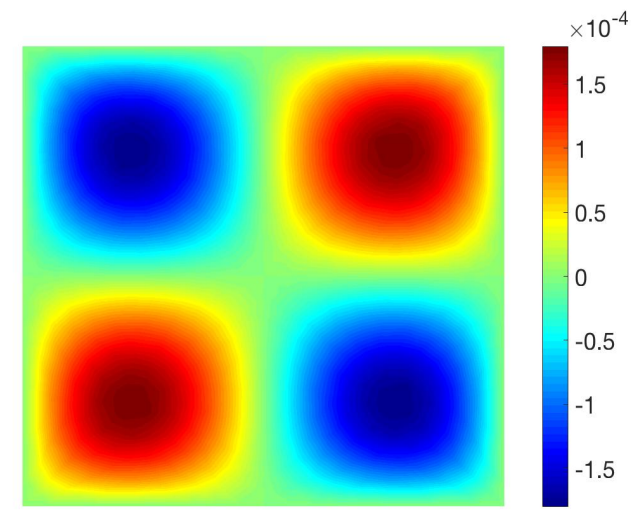

(c) $\hat{\mathbf{u}}_{9}$

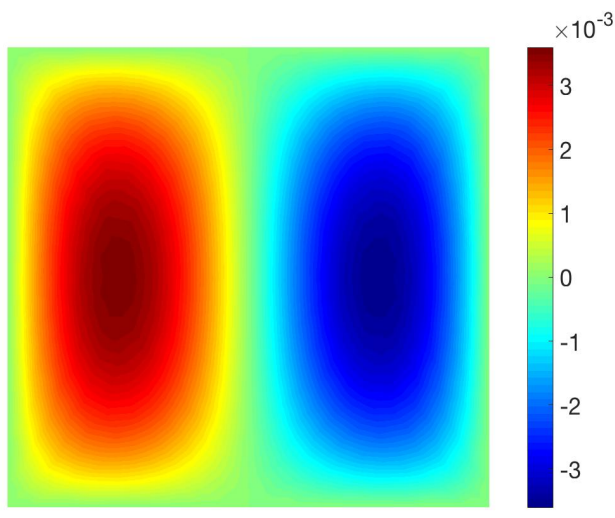

(b) $\hat{\mathbf{u}}_{5}$

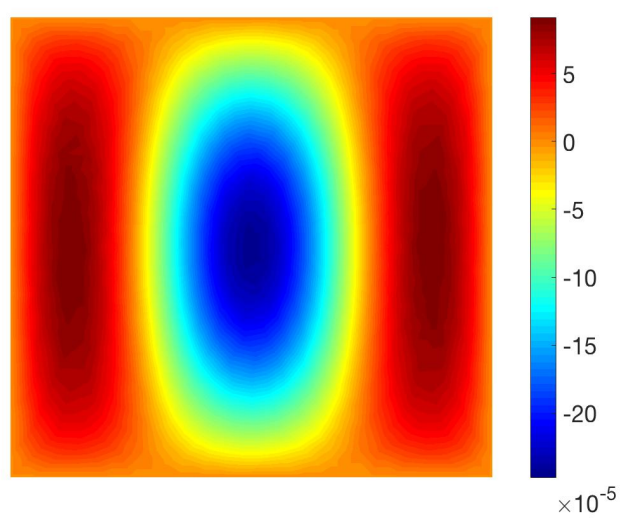

(d) $\hat{\mathbf{u}}_{10}$

Figure B.2: Selected PCE coefficients of the solution field. 


\section{B.3 Non-Intrusive SSFEM}

Performing Galerkin projection onto the PC expansion of solution process given in Eq. (2.10) and then exploiting orthogonality properties of the basis functions, the PCE coefficients of the solution process can be evaluated as follows [1,9, 115];

$$
\hat{\mathbf{u}}_{k}=\frac{\left\langle\mathbf{u}(\theta) \Psi_{k}(\boldsymbol{\xi})\right\rangle}{\left\langle\Psi_{j}(\boldsymbol{\xi}) \Psi_{k}(\boldsymbol{\xi})\right\rangle}=\frac{1}{\left\langle\Psi_{k}(\boldsymbol{\xi})^{2}\right\rangle} \int_{\Omega} \mathbf{u}(\theta) \Psi_{k}(\boldsymbol{\xi}) \mathrm{p}(\boldsymbol{\xi}) d \boldsymbol{\xi}
$$

where $\left\langle\Psi_{j}(\boldsymbol{\xi}) \Psi_{k}(\boldsymbol{\xi})\right\rangle$ is non-zero only for $j=k$ and it can be obtained analytically beforehand, for instance see Table A.4. Therefore, the major computational efforts lies in the evaluation of the multidimensional integral in the numerator of Eq. (B.5) [9, 62, 79, 108, 111].

The efficient evaluation of the multidimensional integral in Eq. (B.5) is key part in the implementation of of this NISP approach especially for the high dimensional stochastic cases. Consider the FE discretization of a stochastic PDE given in Eq. (2.20). Using $\left\{\boldsymbol{\xi}_{1}, \boldsymbol{\xi}_{2}, \ldots, \boldsymbol{\xi}_{n_{s}}\right\}$ (where $\xi_{i}$ is a set of $L$ random variables), the following deterministic system is solved at $n_{s}$ sample points using an existing deterministic solver as a black-box.

$$
\mathbf{A}(\theta) \mathbf{u}(\theta)=\mathbf{f}
$$

Computed $\mathbf{u}(\theta)$ at each sample points are used to calculate the PCE coefficients of the solution process using Smolyak sparse grid quadrature [9,29, 62, 79, 111, 162].

The sparse grid nodal set for $d=2$ and $l=3$ can be written as [12]

$$
\begin{aligned}
\Theta_{l=3}^{(d=2)} & =\bigcup_{\left|l^{\prime}\right| \leq l+d-1}\left(\Theta_{l_{1}}^{(1)} \times \Theta_{l_{2}}^{(1)}\right) \\
& =\left(\Theta_{1}^{(1)} \times \Theta_{1}^{(1)}\right) \quad\left(l_{1}=1, l_{2}=1\right) \\
& \cup\left(\Theta_{1}^{(1)} \times \Theta_{2}^{(1)}\right) \cup\left(\Theta_{2}^{(1)} \times \Theta_{1}^{(1)}\right) \\
& \cup\left(\Theta_{1}^{(1)} \times \Theta_{3}^{(1)}\right) \cup\left(\Theta_{2}^{(1)} \times \Theta_{2}^{(1)}\right) \cup\left(\Theta_{3}^{(1)} \times \Theta_{1}^{(1)}\right),
\end{aligned}
$$


where $l^{\prime}=\left(l_{1} \ldots l_{d}\right)$ with $\left|l^{\prime}\right|=\sum_{i=1}^{d} l_{i}$ (a specific example to obtain the sparse grid nodal set involving $\Theta_{1}^{(1)} \times \Theta_{2}^{(1)}$ is given below). For the implementation of the multidimensional sparse grid the growth rule in one-dimensional quadrature must be defined. In the current implementation, we have used the Gauss-Hermite quadrature rule [12,162]. For further simplification, consider the Gauss-Hermite quadrature rule in one dimension with the nodes and weight specified in Table B.1, for different level of quadrature.

\begin{tabular}{|l|c|c|}
\hline level $l$ & nodes $\Theta_{l}^{(1)}$ & weights $\mathscr{W}_{l}^{(1)}$ \\
\hline$l=1$ & $\{0\}$ & $\{1\}$ \\
$l=2$ & $\{-1,1\}$ & $\{0.5,0.5\}$ \\
$l=3$ & $\{-1.7321,0,1.7321\}$ & $\{0.167,0.667,0.167\}$ \\
\hline
\end{tabular}

Table B.1: Nodes and weights for Gauss-Hermite quadrature in one dimension and third level.

Using Eq. (B.7), Eq. (2.39) and Eq. (2.40), the nodes and weight for $l=3$ and $d=2$ are obtained as shown in Table B.2.

\begin{tabular}{|l|c|}
\hline nodes $\Theta_{2}^{(2)}$ & weights $\mathscr{W}_{2}^{(2)}$ \\
$\{-1.732,0\}$ & 0.167 \\
$\{-1.0,-1.0\}$ & 0.25 \\
$\{-1.0,0\}$ & -0.5 \\
$\{-1.0,1.0\}$ & 0.25 \\
$\{0,-1.732\}$ & 0.167 \\
$\{0,-1.0\}$ & -0.5 \\
$\{0,0\}$ & 1.333 \\
$\{0,1.0\}$ & -0.5 \\
$\{0,1.732\}$ & 0.167 \\
$\{1.0,-1.0\}$ & 0.25 \\
$\{1.0,0\}$ & -0.5 \\
$\{1.0,1.0\}$ & 0.25 \\
$\{1.732,0\}$ & 0.167 \\
\hline
\end{tabular}

Table B.2: Nodes and weights for Gauss-Hermite quadrature in two dimension and third level. 
The relations in Eq. (B.7) are used to get the set of nodes in Table B.2. For instance, the nodes for $\left(\Theta_{1}^{(1)} \times \Theta_{2}^{(1)}\right)$ are obtained by taking tensor produt of $\Theta_{1}^{(1)}=\{0\}$ and $\Theta_{2}^{(1)}=\{-1,1\}$, resulting into the following set of nodes $=[\{0,-1\},\{0,1\}]$ which correspond to the $6^{r d}$ and the $8^{\text {th }}$ row in the first column of Table B.2. The difference relation given in Eq. (2.40) is used to get the corresponding weights [12] (refer to the example given in Sec. 2.2.2 for more details about the weights of the sparse grid quadrature).
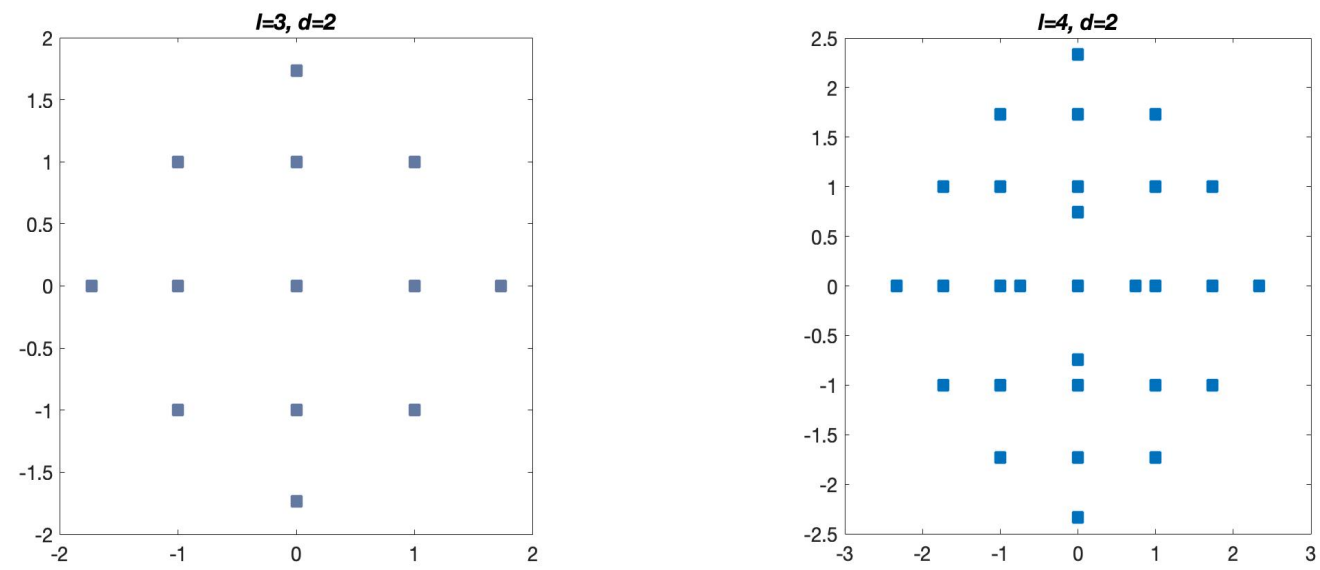

Figure B.3: Two-dimensional sparse grid with $l=3$ and $l=4$.
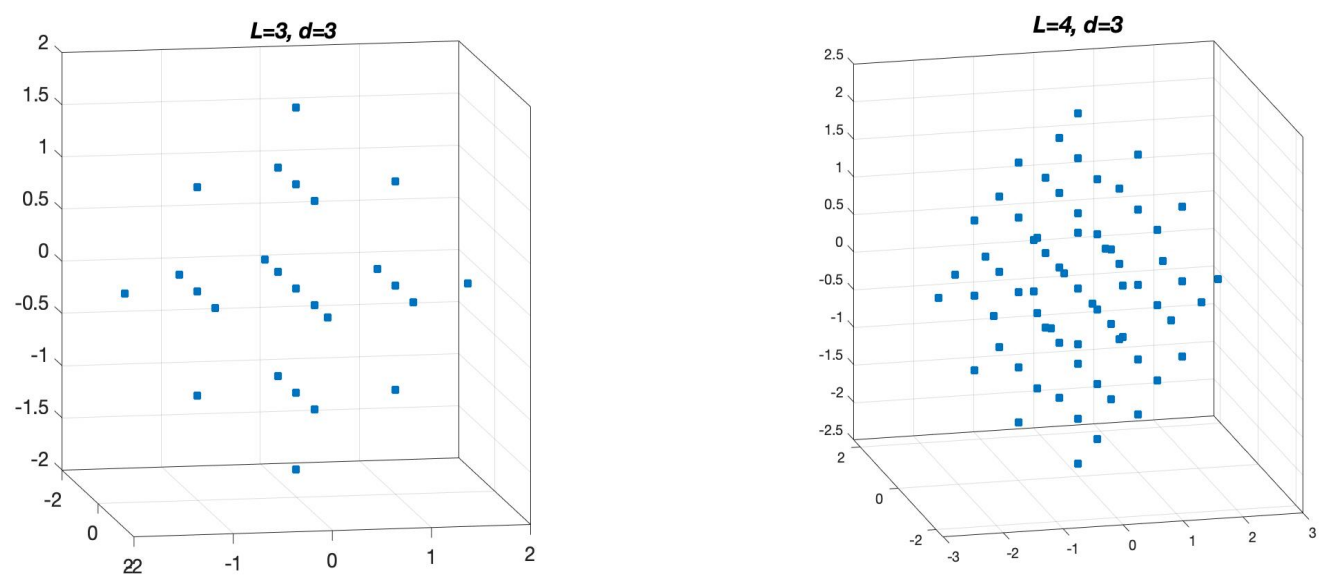

Figure B.4: Three-dimensional sparse grid with $l=3$ and $l=4$. 
The sparse grid in two and three dimensions for $l=2$ and $l=3$ are shown in Fig. B.3 and Fig. B.4, respectively. Using Smolyak sparse grid quadrature with $l=3$ and $d=2$, deterministic FEM sample evaluations are performed using FEniCS package for the stochastic PDE defined by Eq. (2.14). The following numerical parameters $b=1, \sigma=0.3, L=3$, $p_{u}=3$ and $f=1$ are used. The procedure outlined in Algorithm 13 is employed to obtained PCE coefficients of the solution process. A unit square domain, discretized using 600 nodes and 1200 elements is used. The mean and standard deviation of the solution field are plotted in Fig. B.5. A few selected PCE coefficients are plotted in Fig. B.6.

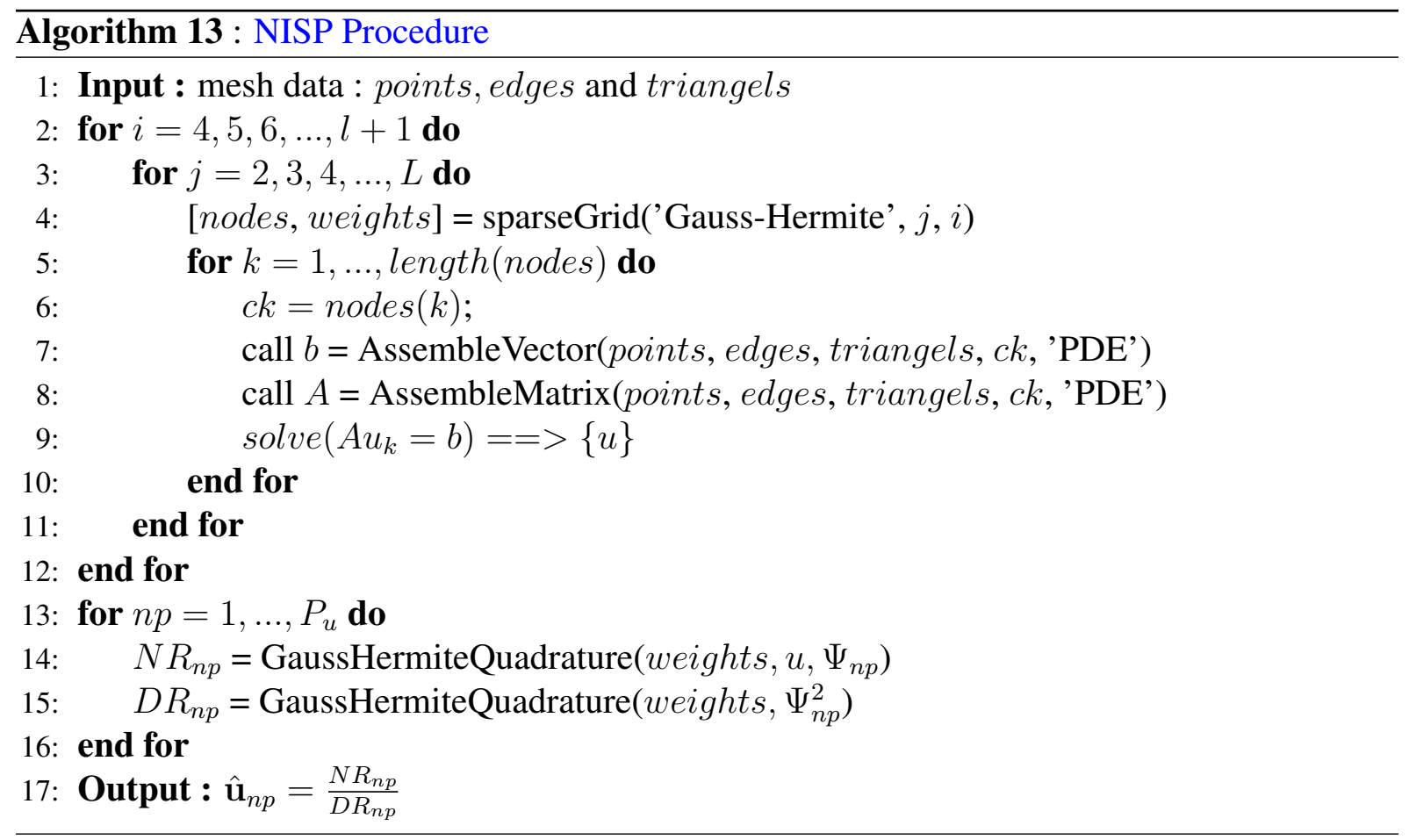



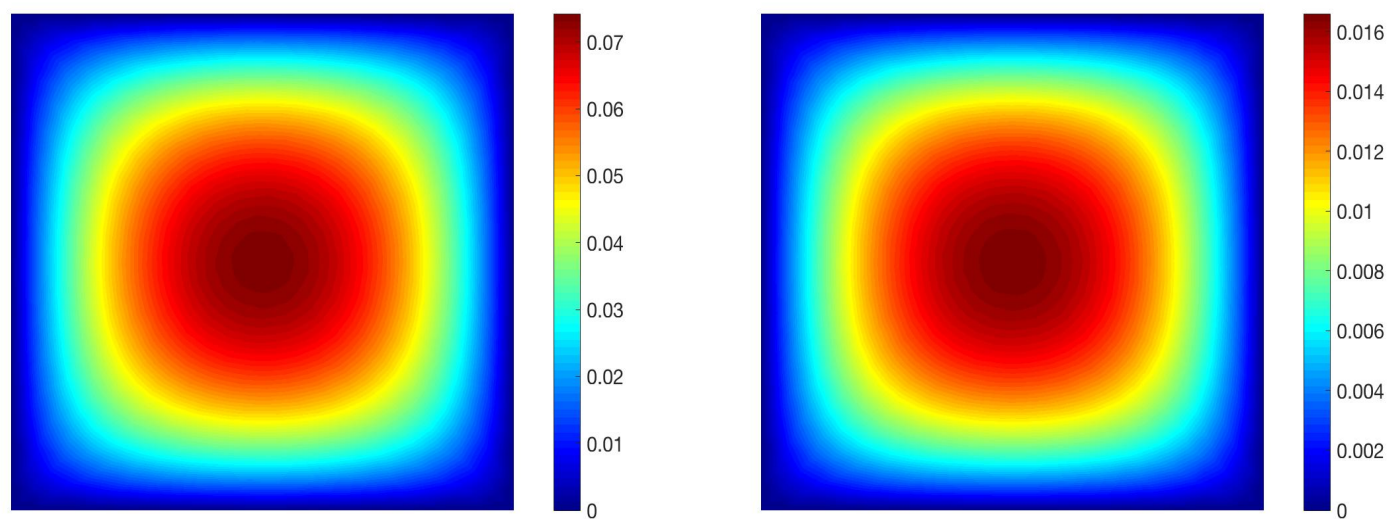

Figure B.5: Mean and standard deviation of the solution field.

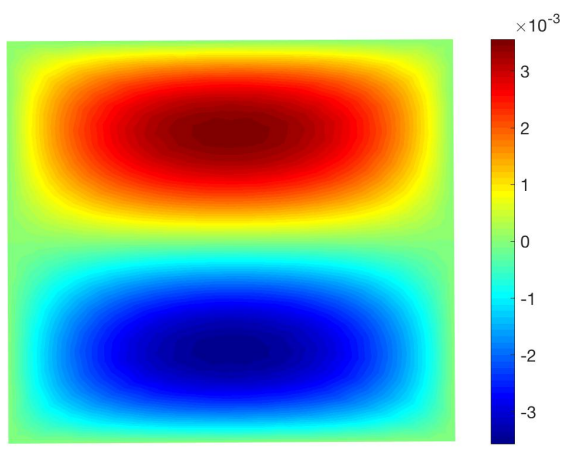

(a) $\hat{\mathbf{u}}_{4}$

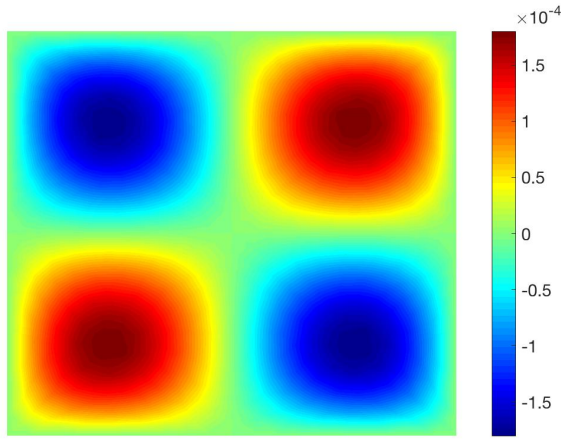

(c) $\hat{\mathbf{u}}_{9}$

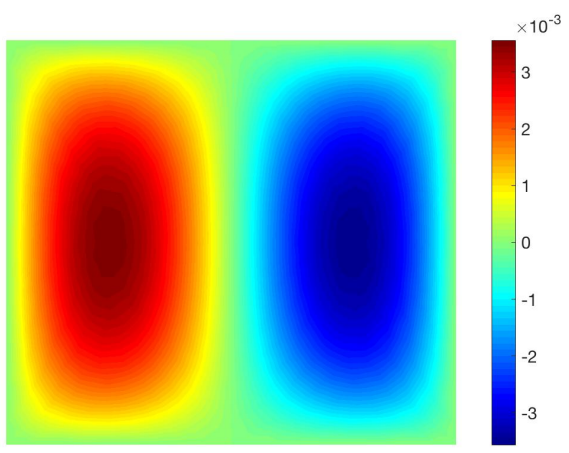

(b) $\hat{\mathbf{u}}_{5}$

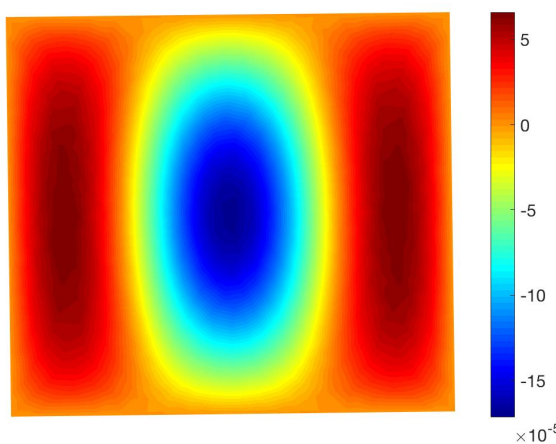

(d) $\hat{\mathbf{u}}_{10}$

Figure B.6: Selected PCE coefficients of the solution field. 


\section{Appendix C}

\section{Numerical Validation of Parallel Domain}

\section{Decomposition Code}

In this section, the numerical validation of the domain decomposition (DD) solver based parallel code developed in this thesis is presented. The output of MPI, FEniCS and PETSc based parallel codes as implemented in Chapter 3 for steady-state stochastic diffusion equations is compared against Monte Carlo simulations (MCS). Also, the parallel code is validated with the SSFEM code developed in [178], which is validated using method of manufactured solution (see Appendix-D.3 in [178] for more details).

A unit square domain, discretized using 1200 elements and 600 nodes is used for simulations. The numerical simulations are performed by using the DD-based parallel NNC/BDDC solver for the stochastic diffusion equations defined by Eq. (2.14). The diffusion coefficient is modeled as a lognormal stochastic process characterized by an underlying Gaussian stochastic process with $\mu=0, \sigma=0.3$ and exponential covariance kernel given in Eq. (A.3) with $b=1$. The PCE for the input stochastic process with two random variables $L=2$ and second order PCE $p_{\mathrm{A}}=2$ is used and the PCE for the solution process with $L=2$ and $p_{u}=3$. The constant source term $f=1$ is used. Similarly 1000 MC simulations are performed using FEniCS deterministic FEM package (running serially without domain decomposition) for the same mesh using same 
numerical parameters. Latin hypercube sampling (LHS) [226] is used for MCS for efficient sampling.

The mean and standard deviation of the solution field obtained by using MCS with 1000 LHS samples and DD-based parallel code for intrusive SSFEM with 12 subdomains are plotted in Fig. C.2a, C.2b, C.2c and C.2d, respectively. The results are identical in both the cases with the maximum error in the standard deviation is in the order of $10^{-5}$. Similarly, we also compare the probability density function using MCS and PCE at the nodes with coordinates $x=0.503, y=0.501$ (near the center of the domain) in Fig. C.1a and $x=0.811, y=0.126$ (towards the upper right corner of the domain) in Fig. C.1b. The probability density functions obtained by MCS and PCE show very good qualitative agreement.

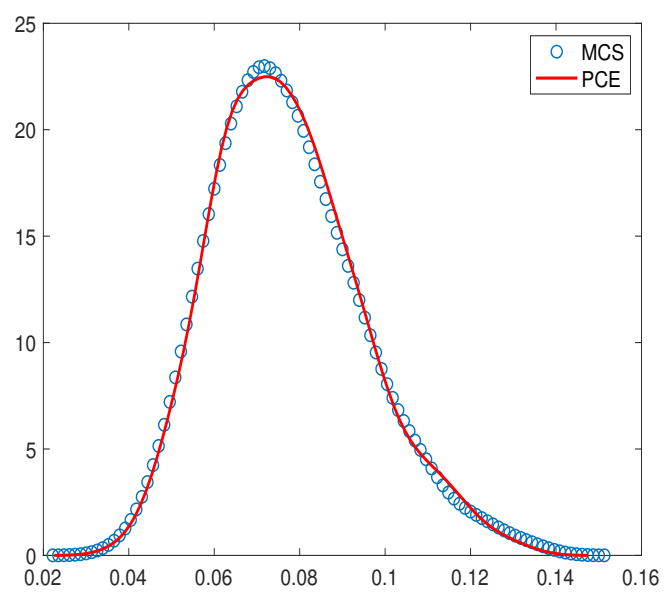

(a) At node with $x=0.503, y=0.501$

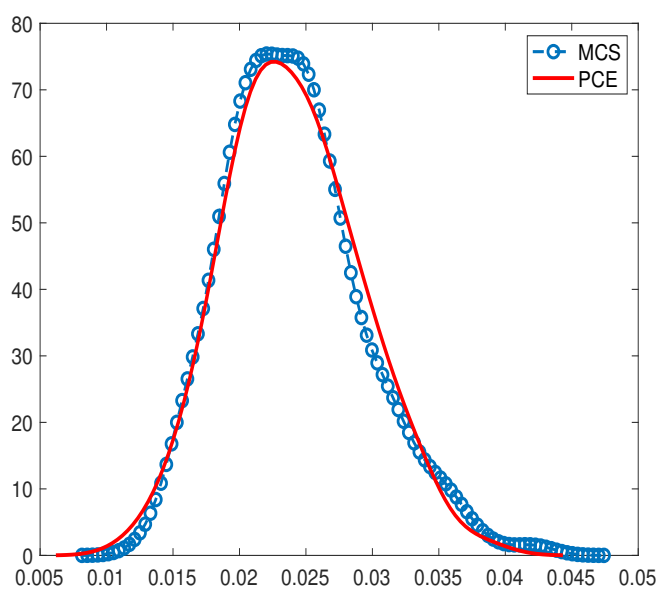

(b) At node with $x=0.811, y=0.126$

Figure C.1: Validation of parallel NNC/BDDC-PCGM solver against MCS: the probability density function of the solution process at two nodal points.

Note that for some applications only qualitative comparisons (also referred to as viewgraph norm) are not sufficient and other measures of comparisons would be required as pointed out by Oberkampf et al. [47,227]. Also, note that these results involve a single experiment of MCS. Evidently, such comparison depends on a particular MCS experiment. However, a large number of MC samples is included in this experiment and hence the results from other MCS 
experiments show similar trend (but not included for brevity). Furthermore, the convergence behavior of both MCS and PCE will generally be different at the middle and at the tail of the distribution. Depending on the number of expansion terms used and the inherent limitations of PCE (i.e., mean squares convergence property) and the number of samples used in the MCS, may lead to inaccurate results in estimating the tail of the distribution dictating extreme (rare) events. However, as the intended applications of these algorithms, for instance, will be in the state and parameter estimations of the system (using PCKF) and the global sensitivity analysis, such limitations will have a less significant impact.

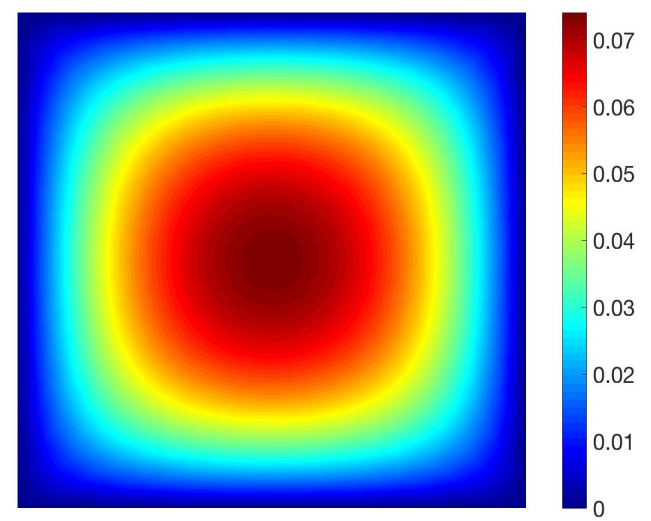

(a) Mean using PCE

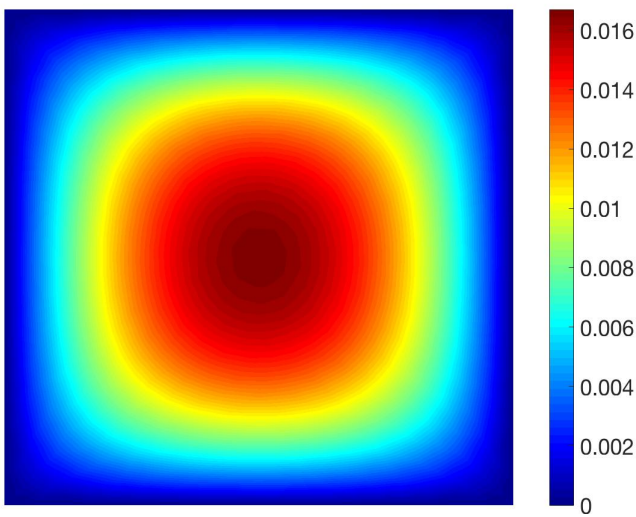

(c) Standard deviation using PCE

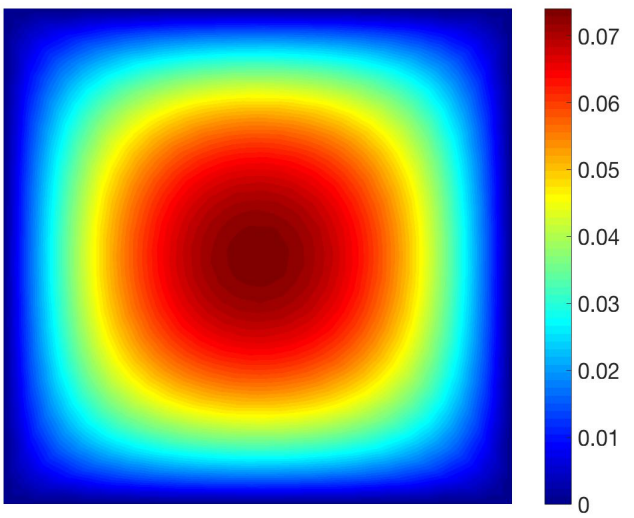

(b) Mean using MCS

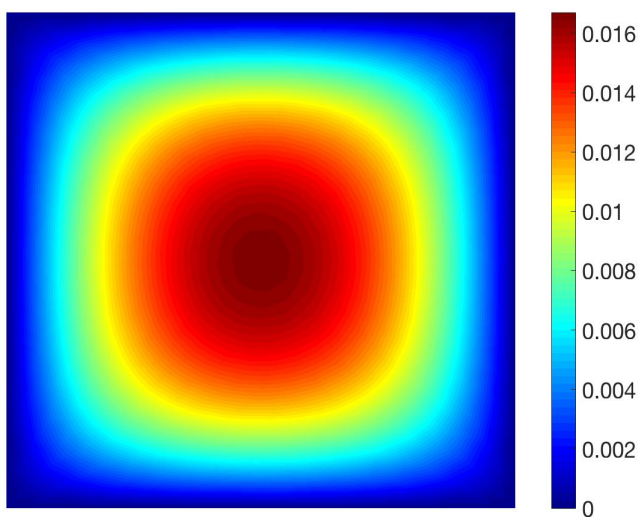

(d) Standard deviation using MCS

Figure C.2: Validation of parallel NNC/BDDC-PCGM solver against MCS: the mean and standard deviation of the solution process (over the entire domain) using parallel solvers and MCS, respectively. 


\section{Appendix D}

\section{Mesh Decomposition in Two and Three}

\section{Dimensions}

In this appendix, we present the implementational details and code snippets of mesh decomposition for the non-overlapping domain decomposition methods. Although, in this thesis, main attention is given to devise scalable parallel domain decomposition based solvers for stochastic PDEs, these solvers and the associated preconditioner rely on mesh decomposition. Therefore, extracting information about nodes, for instance, interfaces, vertex and wirebasket nodes, is the primary requirement. Mesh decomposition, as outlined in Fig. 1.2, is the part of preprocessing step of the domain decomposition based SSFEM solvers package developed, in part, in this thesis. The high-level implementational framework of mesh decomposition is presented in Fig. D.1.

Consider a unit square domain defined using a geometry file compatible with GMSH version2.8.5 [27] as shown in listing D.1. Using GMSH with METIS graph partitioner [28] the mesh is partitioned into, for example, four subdomains as shown in Fig. D.2. A typical mesh file with the information about elements and its partitions is shown in listing D.3. The information about the partitions extracted from the mesh file, such as number of partitions an element belongs to and the partition identities, are used to gather local and global interface nodes. 


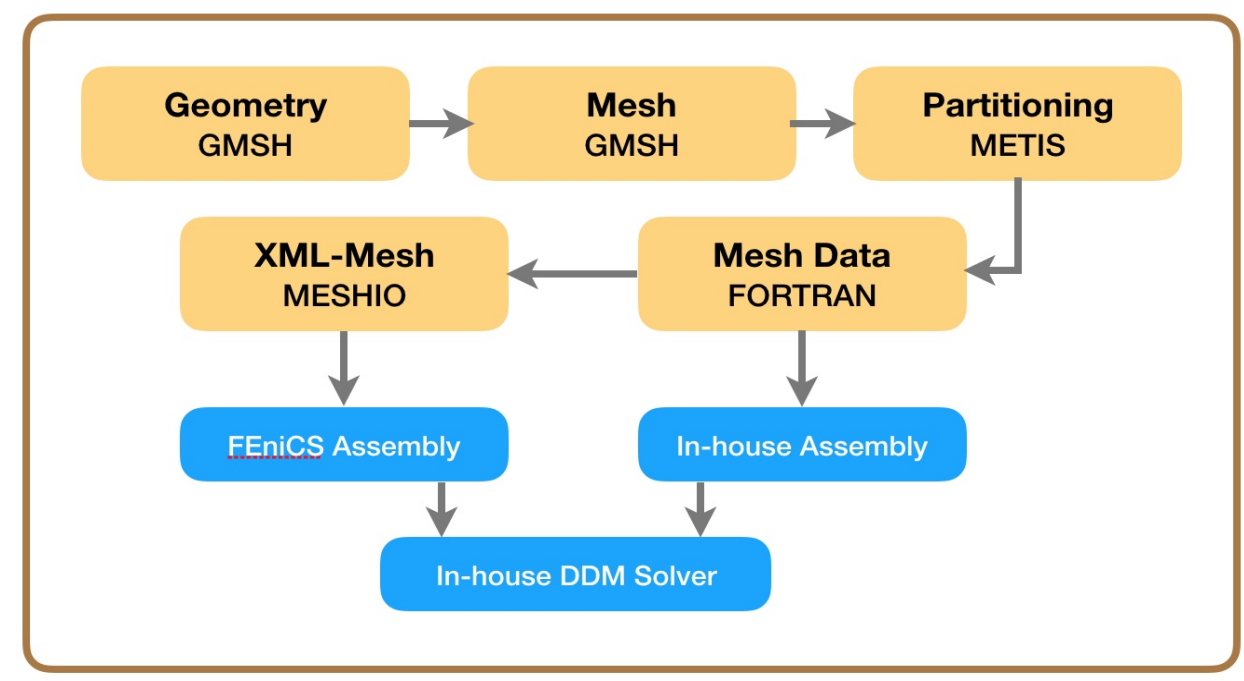

Figure D.1: Implementational framework for mesh partitioning for non-overlapping domain decomposition methods.

Listing D.1: Two-dimensional geometry .geo file.

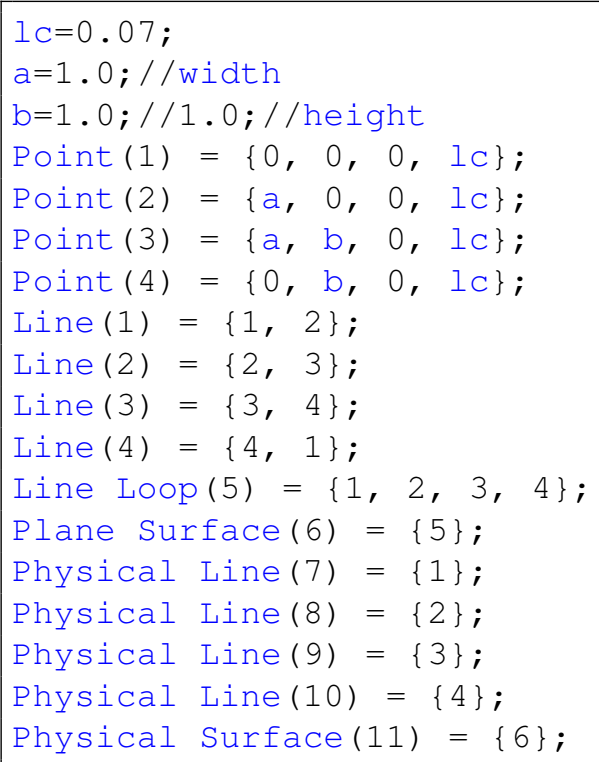

Listing D.2: Three-dimensional geometry .geo file.

$l_{C}=0.1 ;$

$a=0.2 ; / /$ width

$\mathrm{b}=0.2 ; / /$ height

$\mathrm{c}=1.0 ; / /$ length 


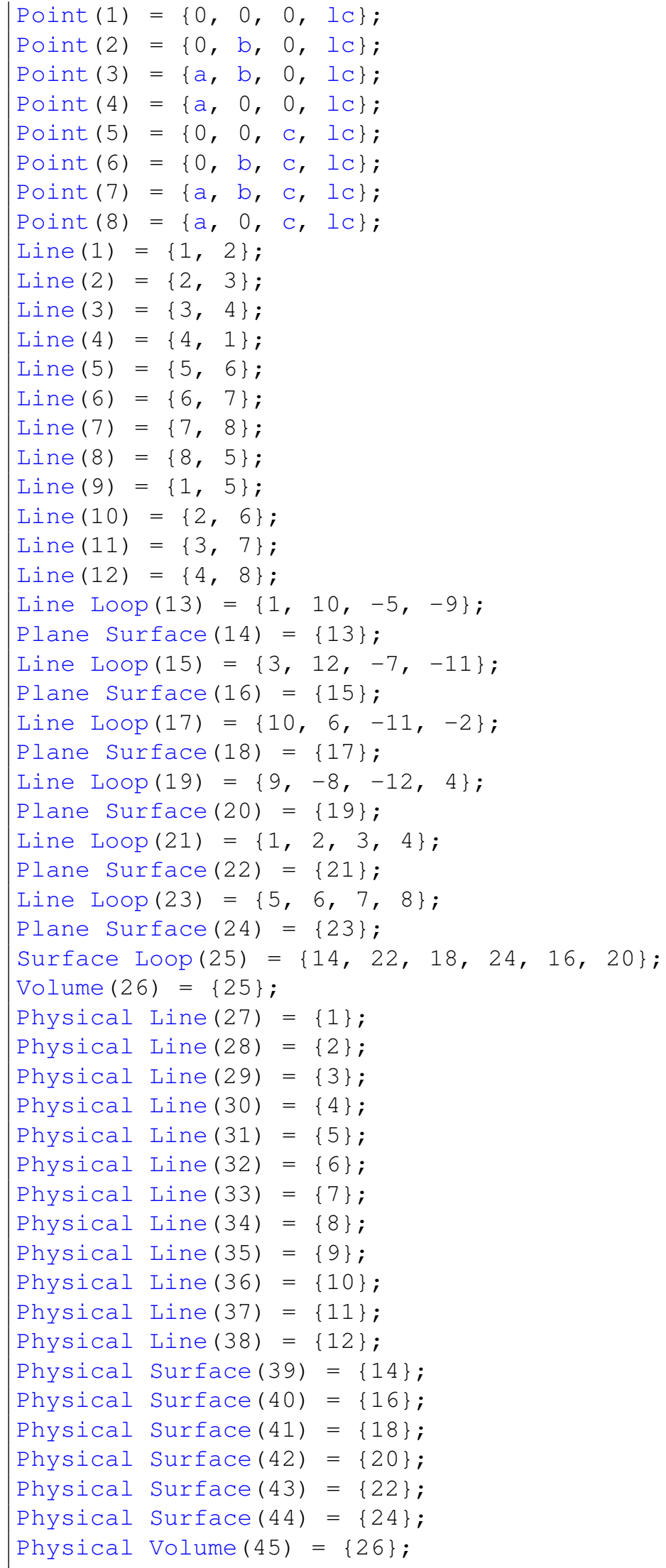


Listing D.3: A typical two-dimensional mesh .msh file.
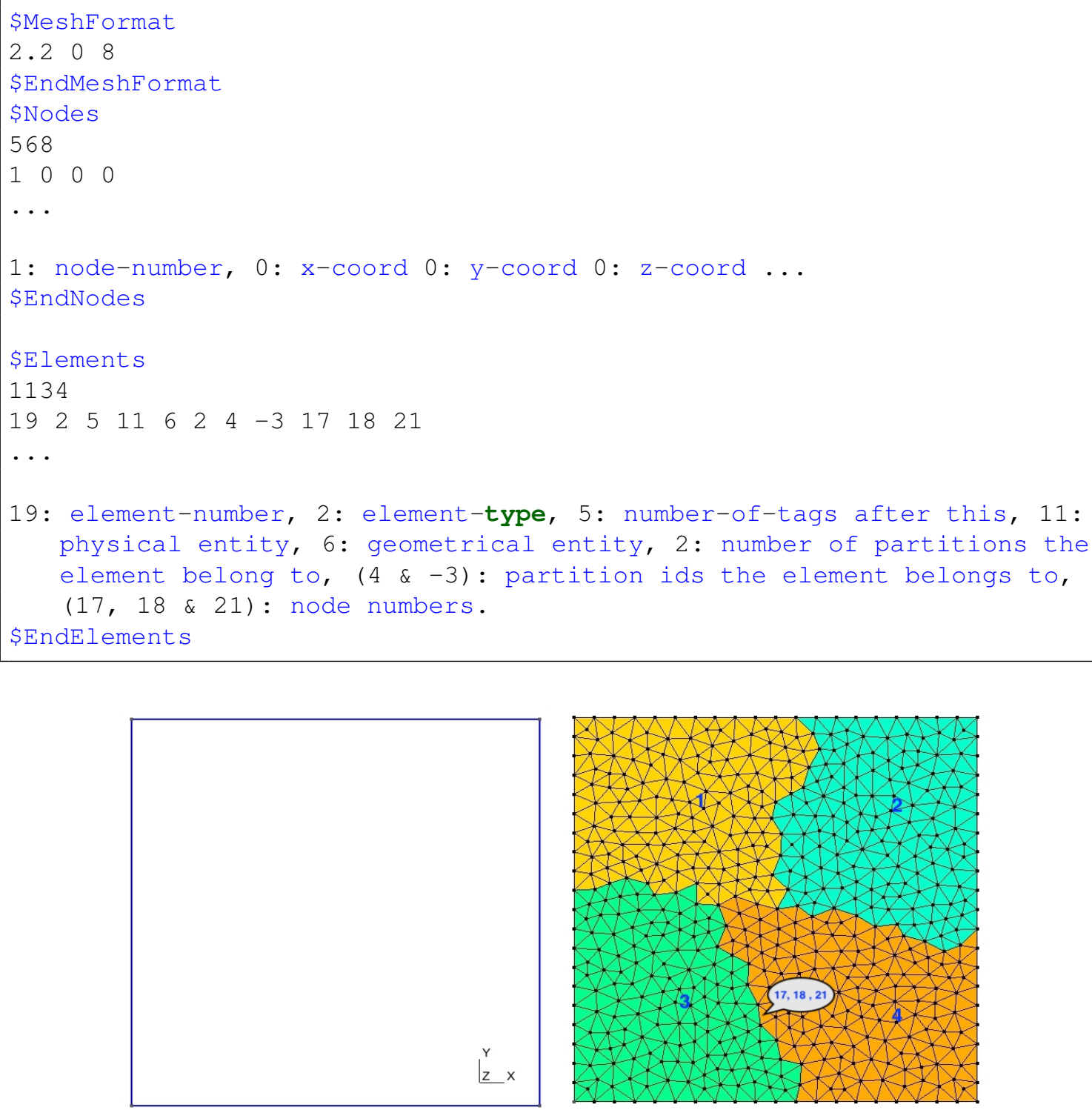

Figure D.2: A typical geometry and mesh for square domain partitioned into four subdomains highlighting the nodes and elements.

For example, element number 19 with nodes 17, 18, 21 as highlighted in Fig. D.2, belongs to the partition with id 4 and shared wit the partition with id 3. It is two-dimensional element on physical surface with tag 11 and geometrical surface with tag 6 (see geometry file listed in D.1). Similarly, the geometry file for a three-dimensional computational domain, for instance, a rectangular beam used in this thesis, is given in the listing D.2. The decomposed two-dimensional and three-dimensional mesh highlighting each subdomains are shown in Fig. D.3. 


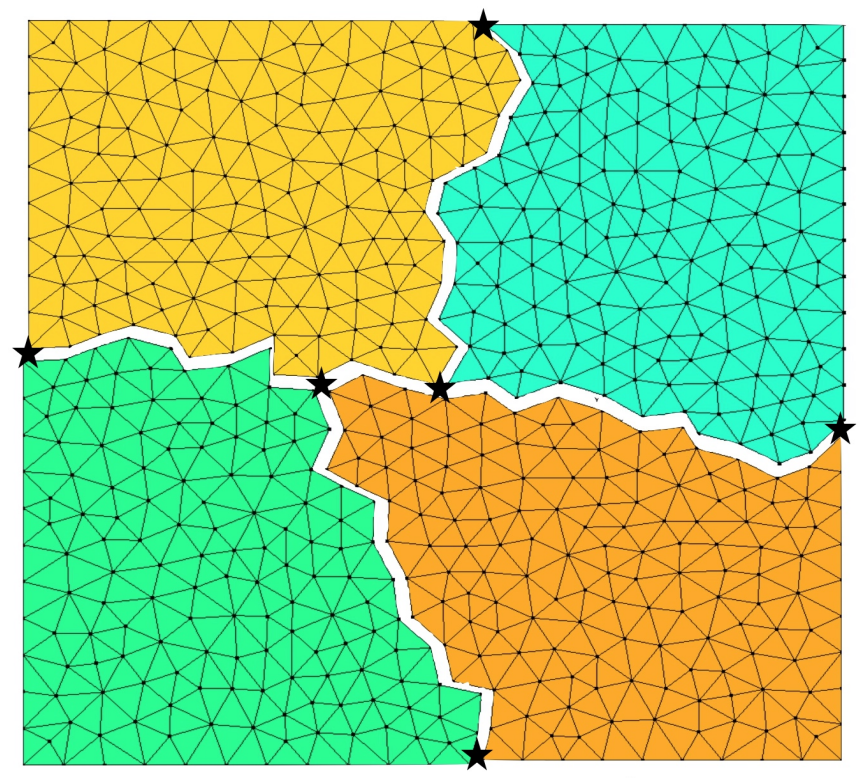

Figure D.3: A typical mesh for square domain partitioned into four subdomains highlighting corner nodes as $(\star)$.

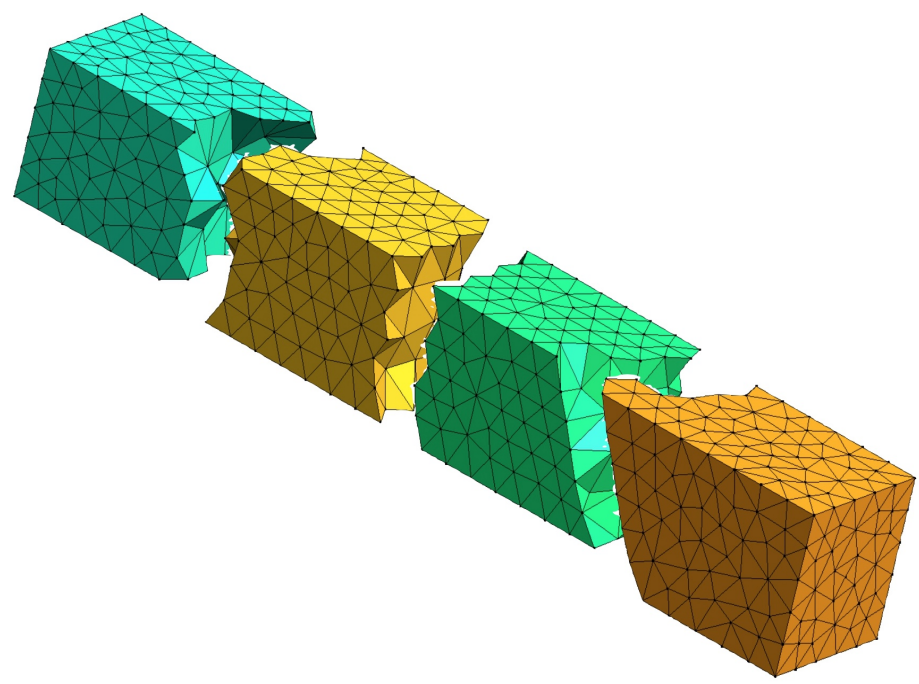

Figure D.4: A typical mesh for rectangular beam partitioned into four subdomains. 
From the partitioned mesh file, such as listed in D.3, we extract the following information at the global-level (for the whole mesh) and local-level (for each subdomain): points, edges, triangles, boundary nodes(bnodes), corner nodes(cnodes), remaining nodes(rhodes) in two and three-dimensions and tetrahedrons in three-dimensions. For the decomposition, we use number of partitions the element belong to and partition ids the element belongs from the GMSH file. The mesh decomposition process is broadly categorized into four steps as follows.

- Global decomposition, i.e., extract data files for the whole mesh: Code snippet listed in D.4 can be used for global decomposition. In the Part 1 of this listing we read the GMSH-mesh file. Next, we extract (global) nodes, points, edges and triangles in the Part 2. This is followed by the procedure to extract interface (boundary) nodes information in the Part 3. Finally, in the Part 4 of the listing the extracted data files are exported.

- Local decomposition, i.e., extract data files for each subdomains: Code snippet listed in D.5 can be used for local decomposition. Here, we first import the mesh file and the global mesh data files (extracted using the listed in D.4) in the Part 1 of the listing. In the Part 2 of the listing the global interface nodes for each partition are separate based on the partition information from the mesh file. This is followed by the procedure to separate subdomain-level (local) nodes, points, edges and triangles in the Part 3. Finally, extracted subdomain-level data files are saved.

- Corner and remaining nodes, i.e., extract vertices and remaining nodes data at global and subdomain-level: Code snippet listed in D.6 can be utilized. In the Part 1 of this listing we import the mesh data extracted in global and local mesh decomposition procedure used listed in D.4 and D.5, respectively. Next, in the Part 2 and 3 of the listing the (local) interface nodes, points and elements are separated into corner nodes (vertex) and remaining nodes, respectively (see Chapter 3 for definition of the corner and remaining nodes). Finally, in the Part 4 of the listing, we extract the global corner and remaining nodes and export the data files. 
- Rearranging and renumbering, i.e., rearranging and renumbering of nodes, points, elements and triangle for DDM compatibility: Code listed in D.7 can be employed for this purpose. In the Part 1 of this listing, we import the required mesh data. Next, in the Part 2 of the listing the re-arrangements of the boundary nodes, nodes and points using corner and remaining nodes are performed. For example, for a given subdomain the local boundary nodes are re-arranged as the local remaining nodes on top, followed by the local corner nodes. In the Part 3 of this listing, the re-numbering of re-rearranged nodes, boundary nodes, points, edges and triangles is performed.

For simplicity, the Matlab scripts developed for the decomposition of two-dimensional mesh are listed below. However, for high-resolution meshes used, for instance, mesh with 121896 nodes and 243794 elements partitioned into about 4000 of subdomains, (see Chapter 3), the Fortran scripts are employed for computational efficiency.

Using these listings, the decomposed mesh data files at global and subdomain-level are generated. For instance, points.dat, edges.dat, triangles.dat and other data files generated in the mesh decomposition process are listed in Fig. D.5. The data files for each subdomains can be directly utilized with an in-house stochastic FEM assembly package for DD-based SSFEM solver. However, to use FEniCS-based stochastic assembly, we need to create FEniCS compatible mesh file. Although, FEniCS is compatible with many mesh formats, in this thesis, we have used $X M L$ format [177].

Python code snippet to convert mesh data from Fig. D.5 to FEniCS-xml data for each subdomain is listed in D.8. In the Part 1 of this listing the MPI modules are imported and initialized. In the Part 2 and 3 of this listing the points and elements files for each subdomain are converted to FEniCS compatible $X M L$-format, respectively. Finally in the Part 4 the $X M L$-format mesh file for each subdomain is written. A typical FEniCS compatible $X M L$ mesh file for a subdomain is listed in D.9. 


\section{Listing D.4: Matlab code snippet for global decomposition.}

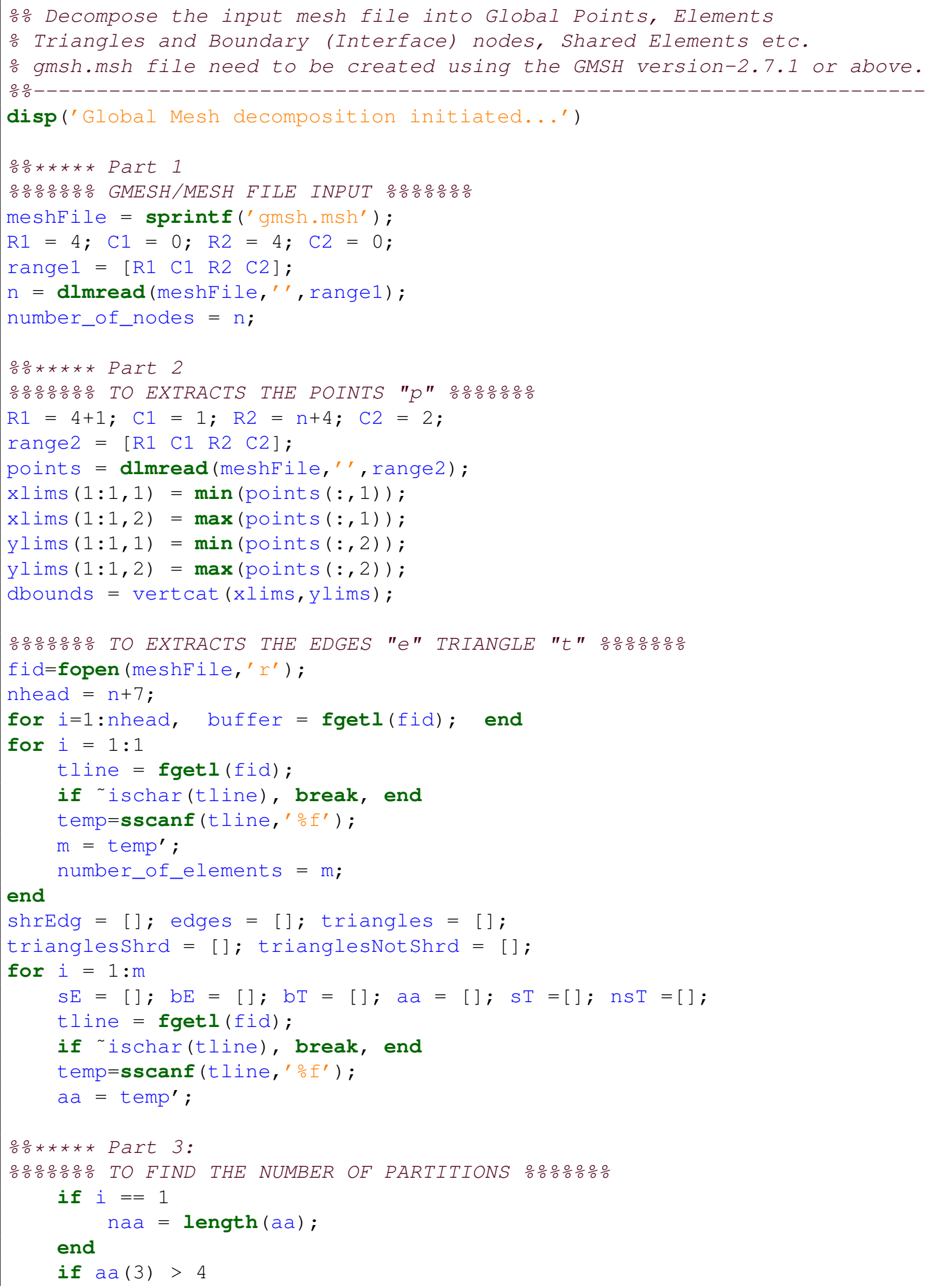




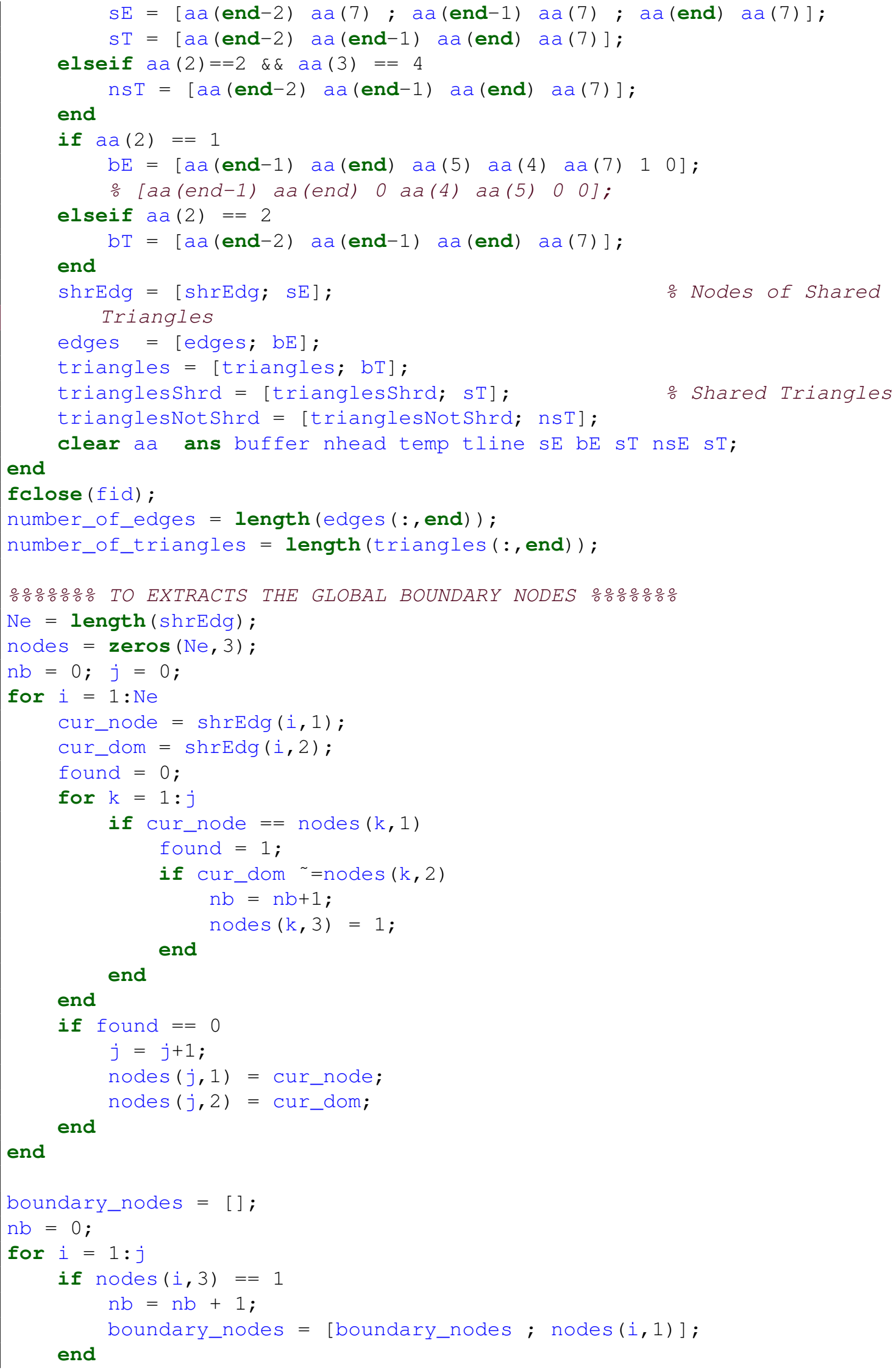




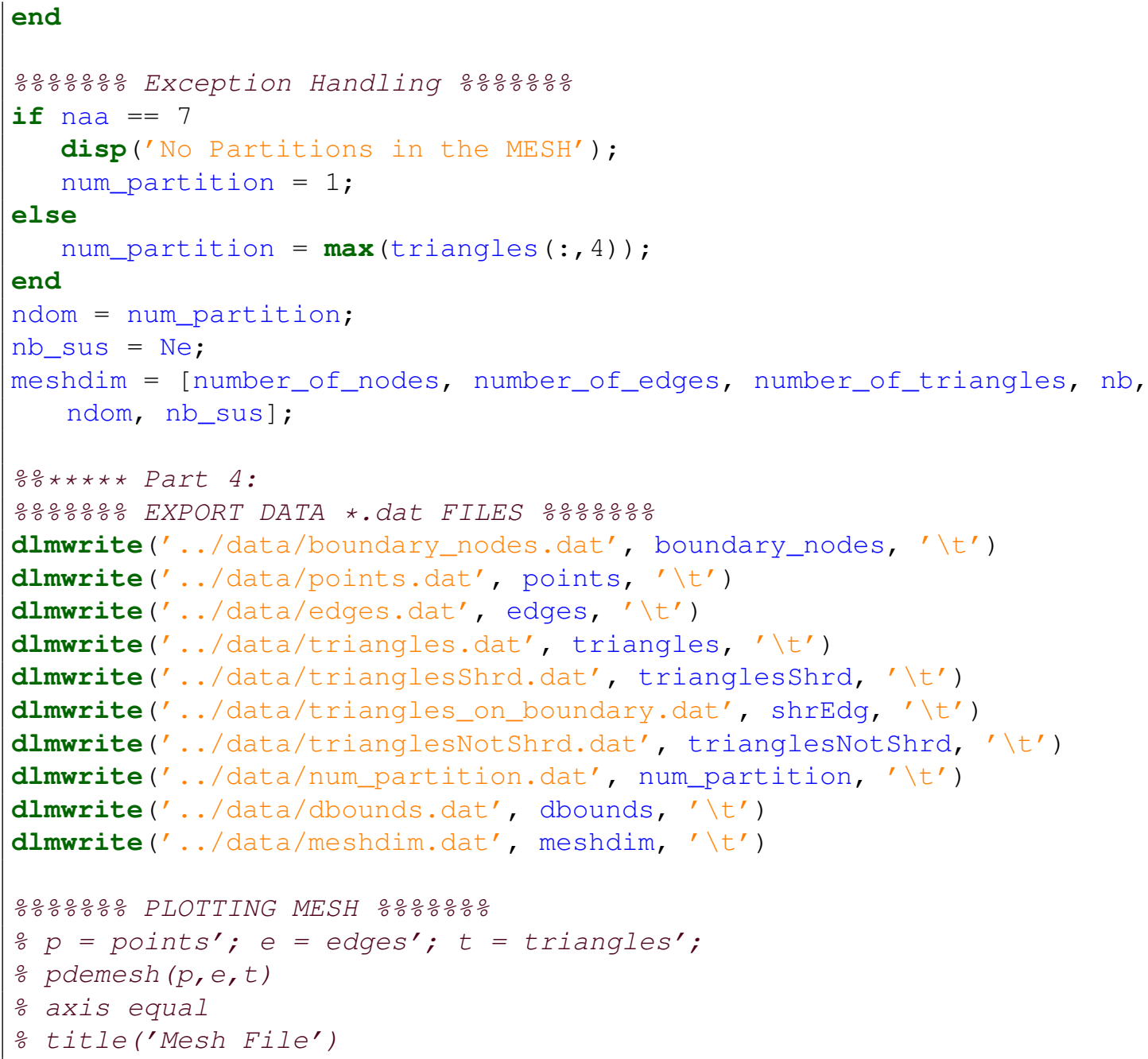

\section{Listing D.5: Matlab code snippet for local decomposition}

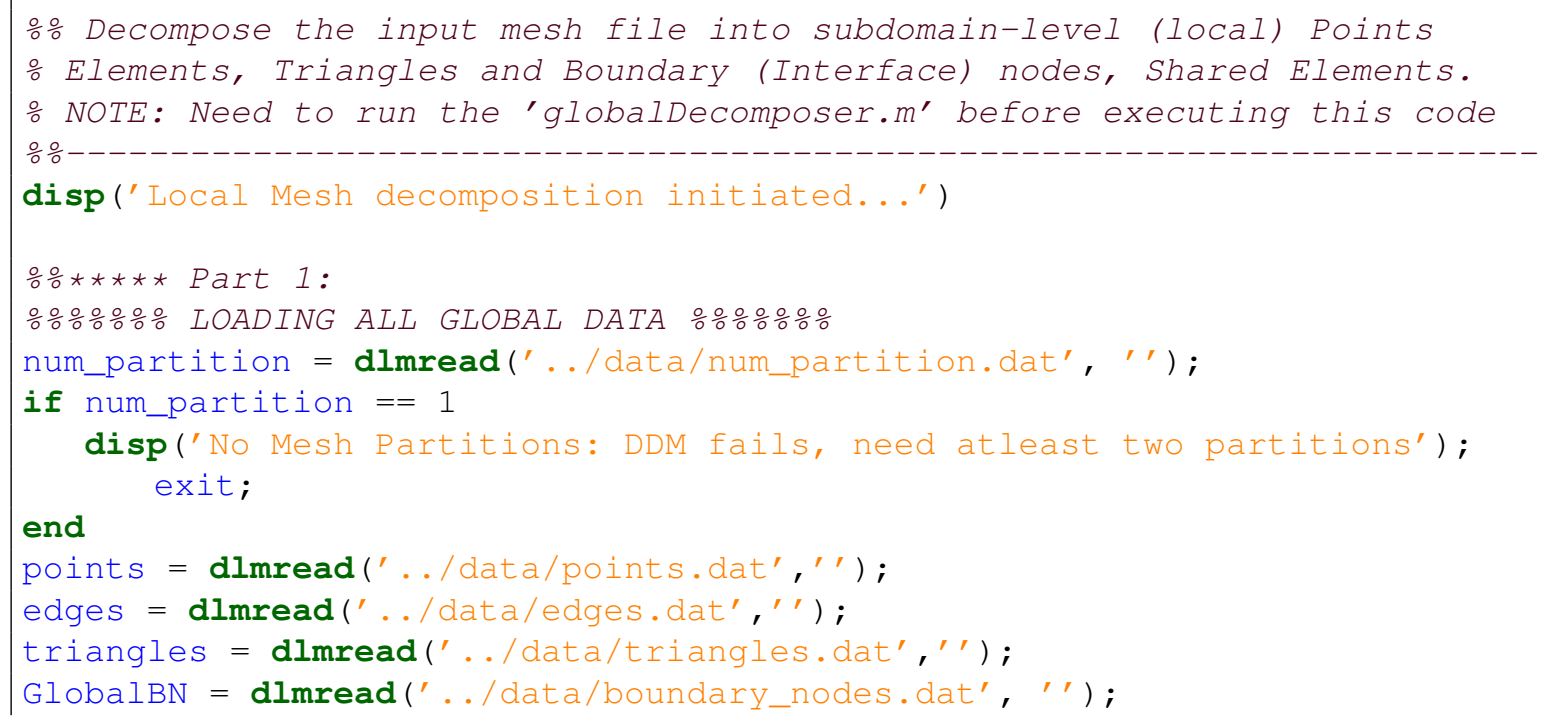




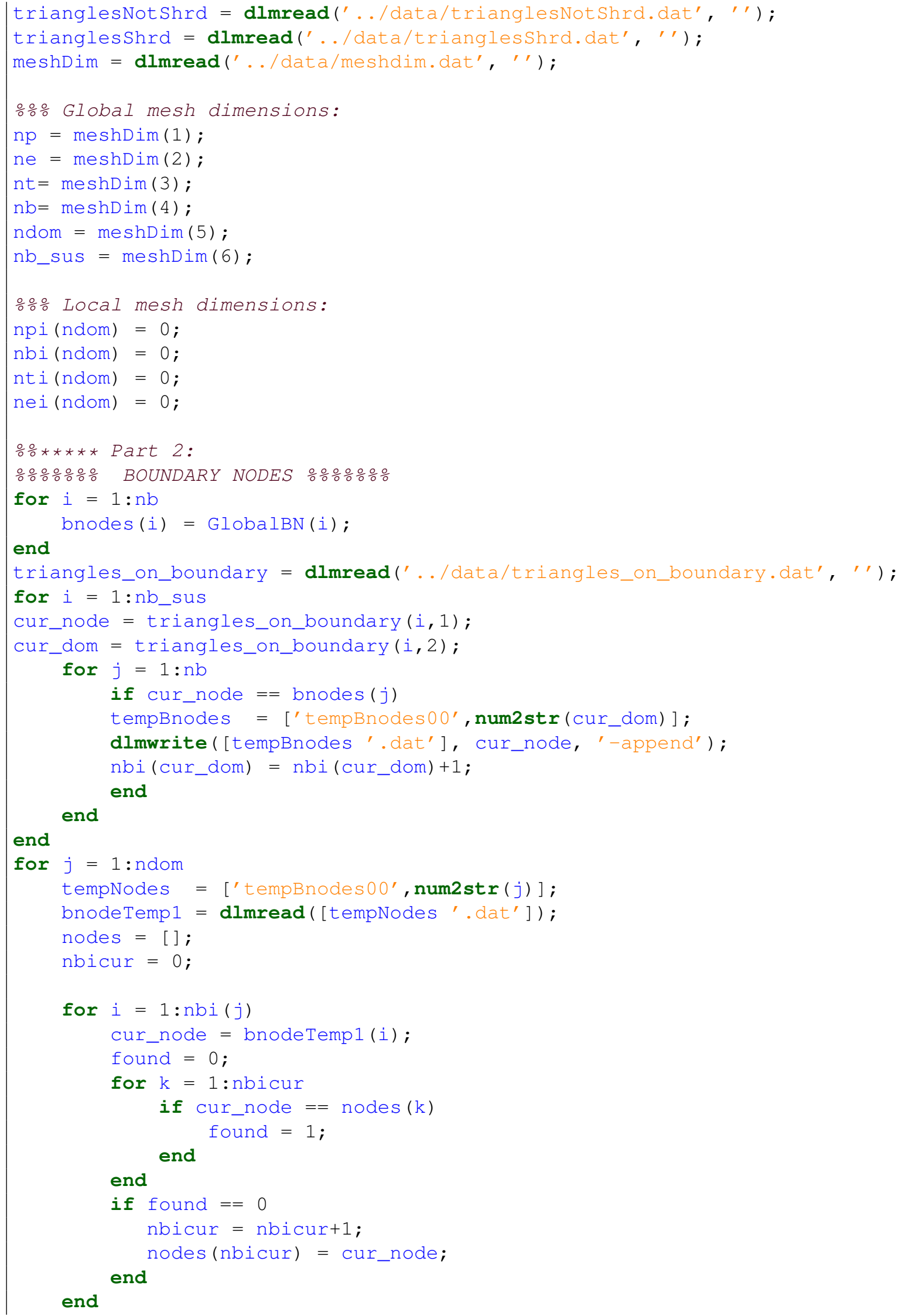




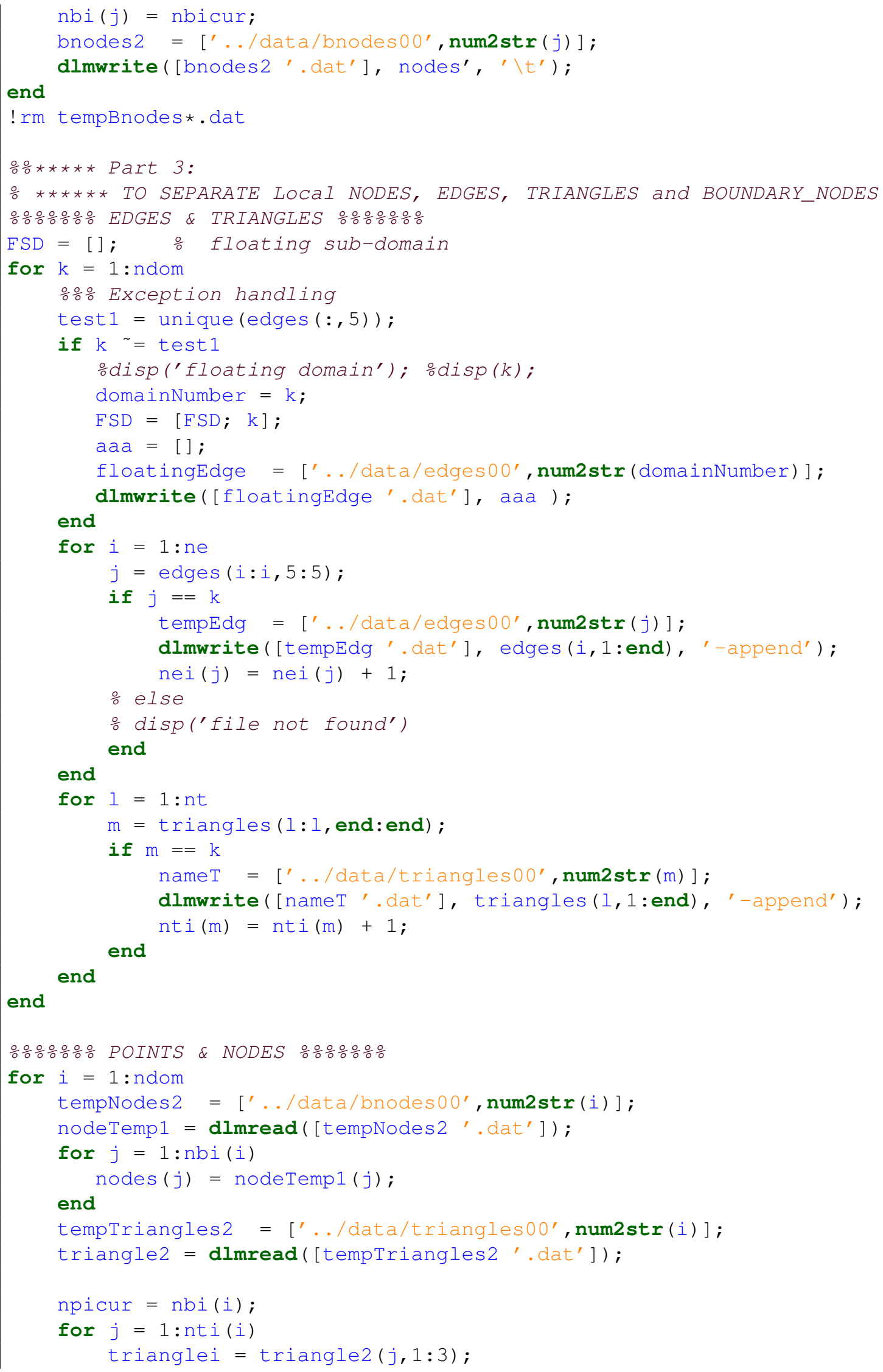




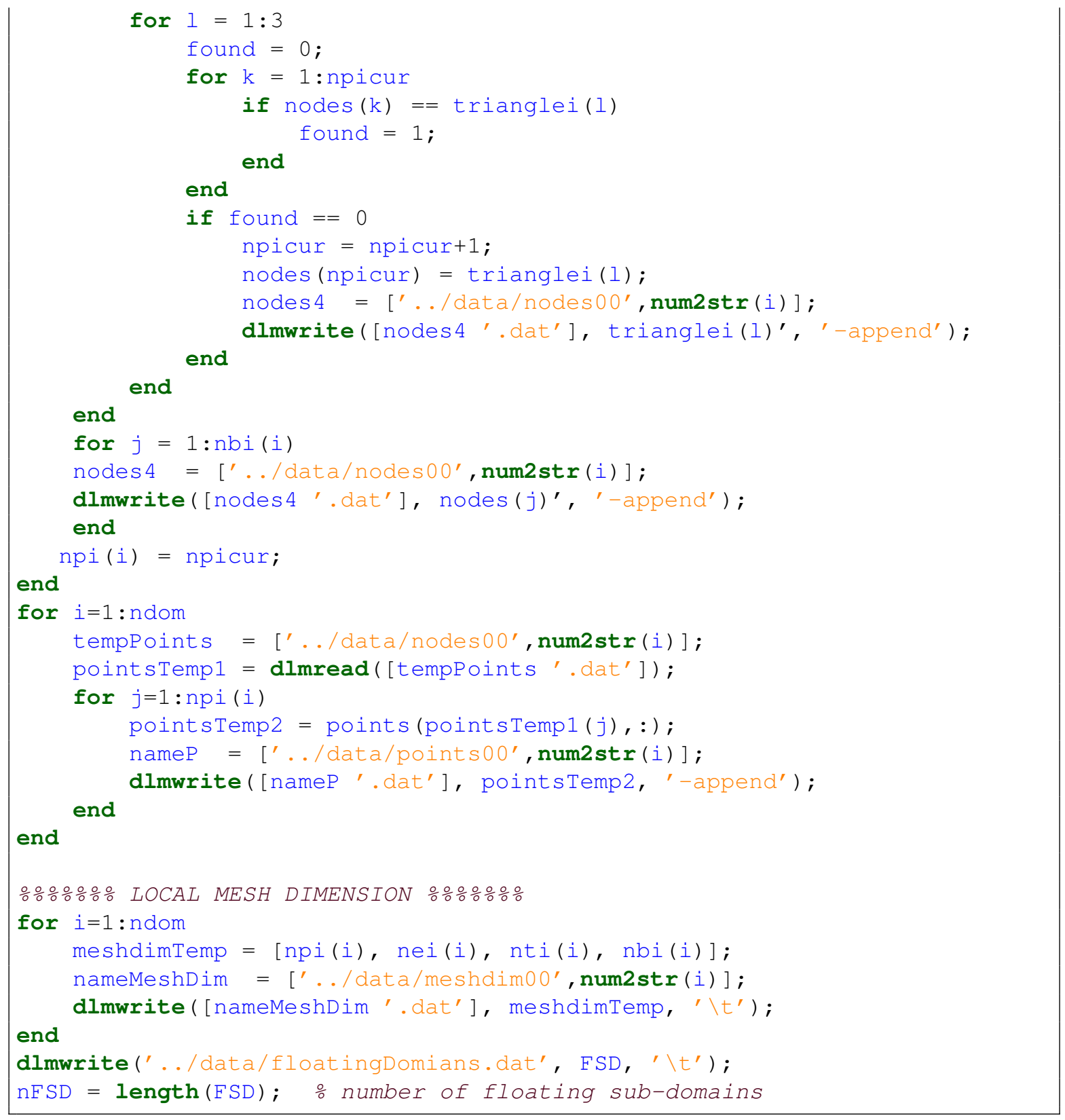

\section{Listing D.6: Matlab code snippet to extract corner and remaining nodes.}

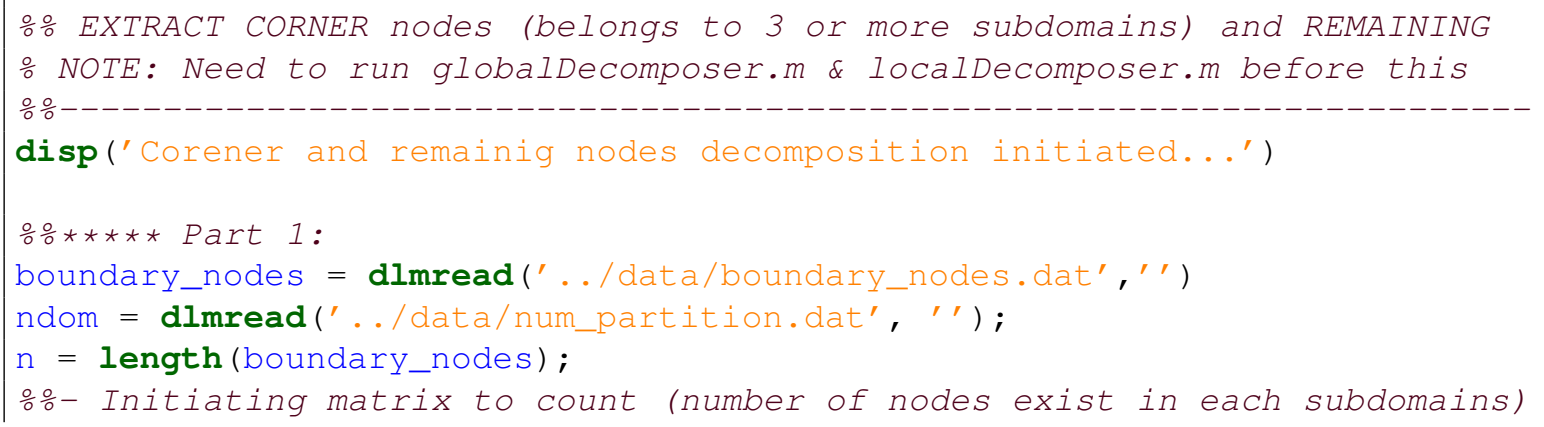




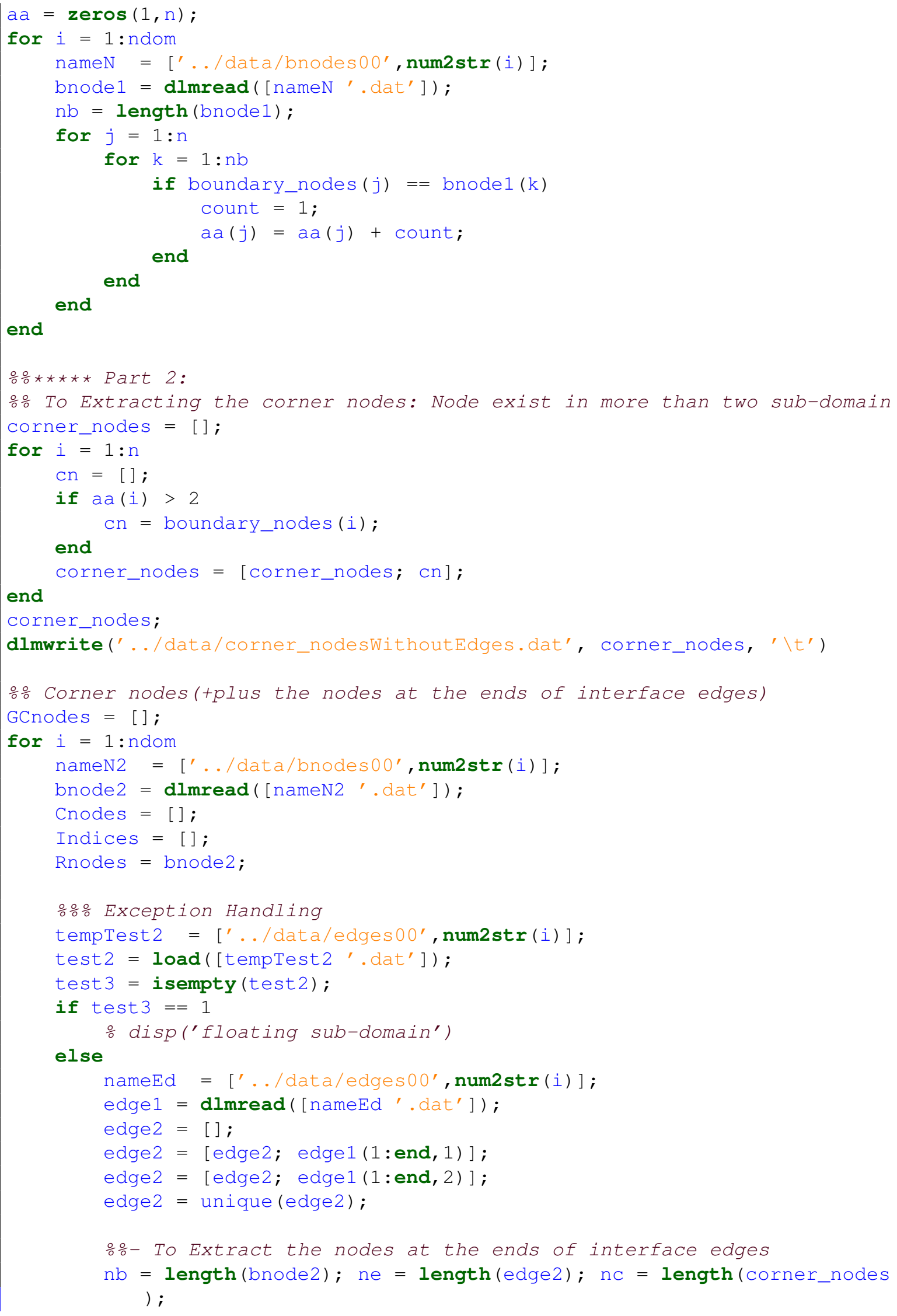




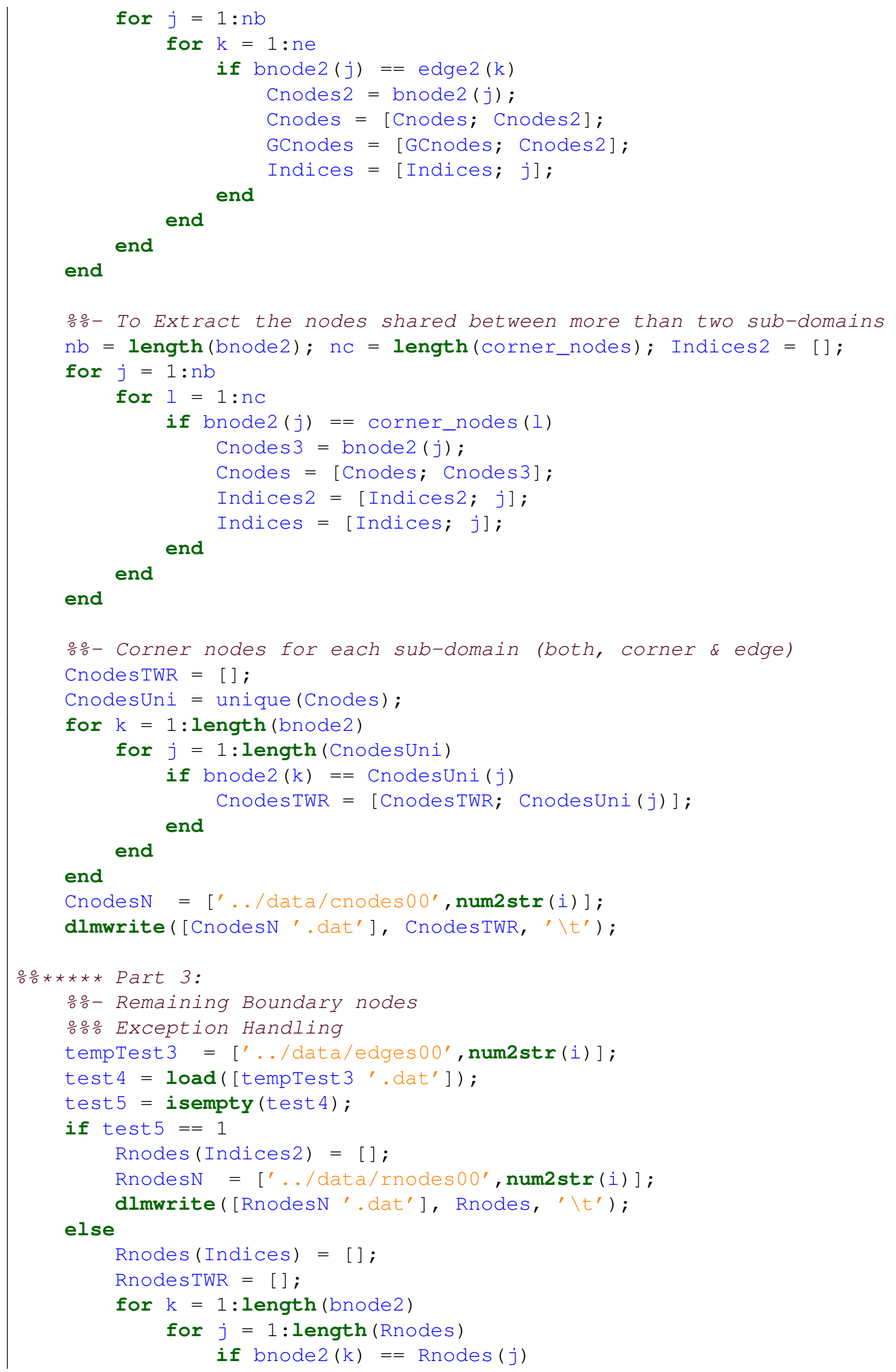




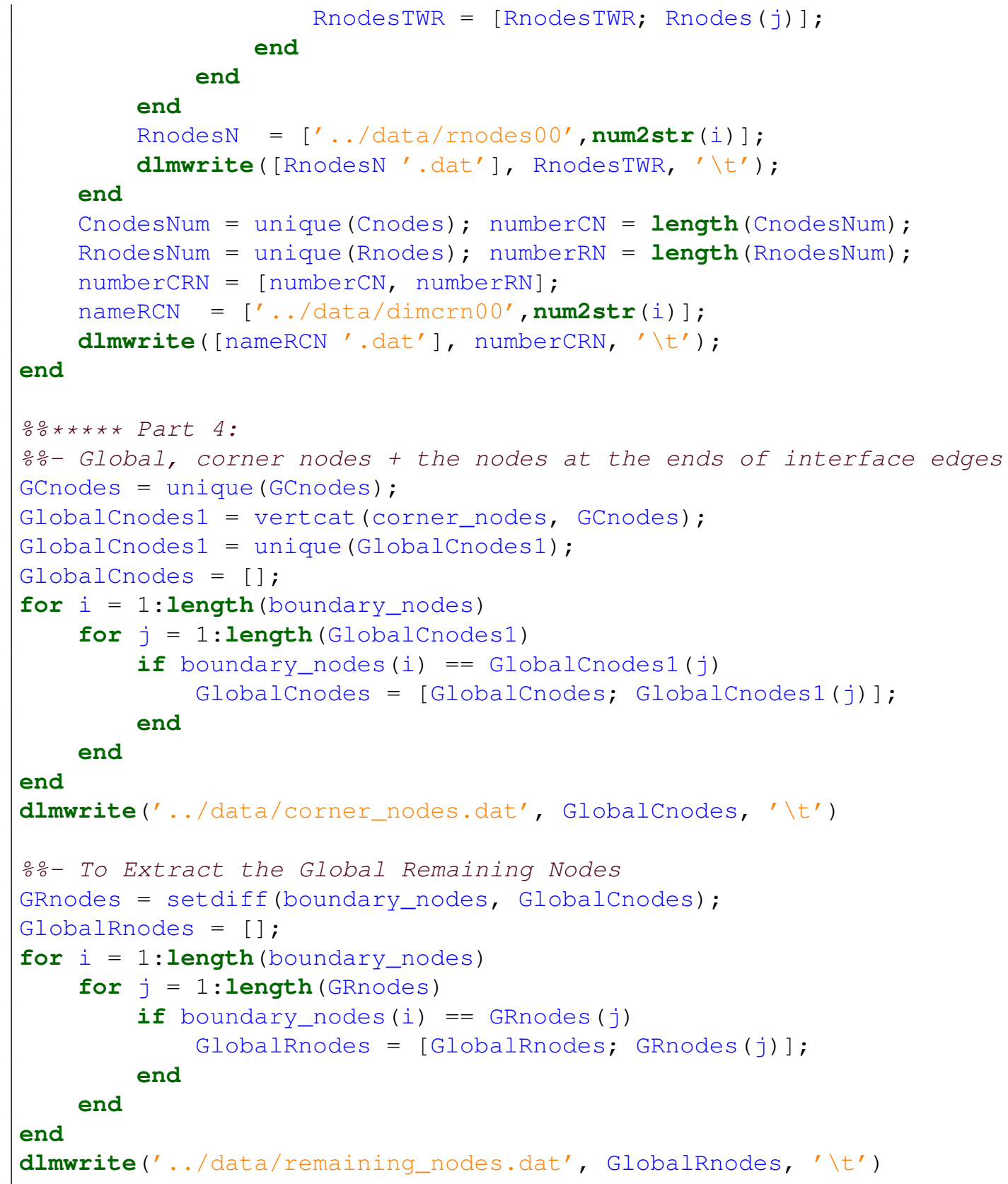

\section{Listing D.7: Matlab code snippet for node rearranging and renumbering.}

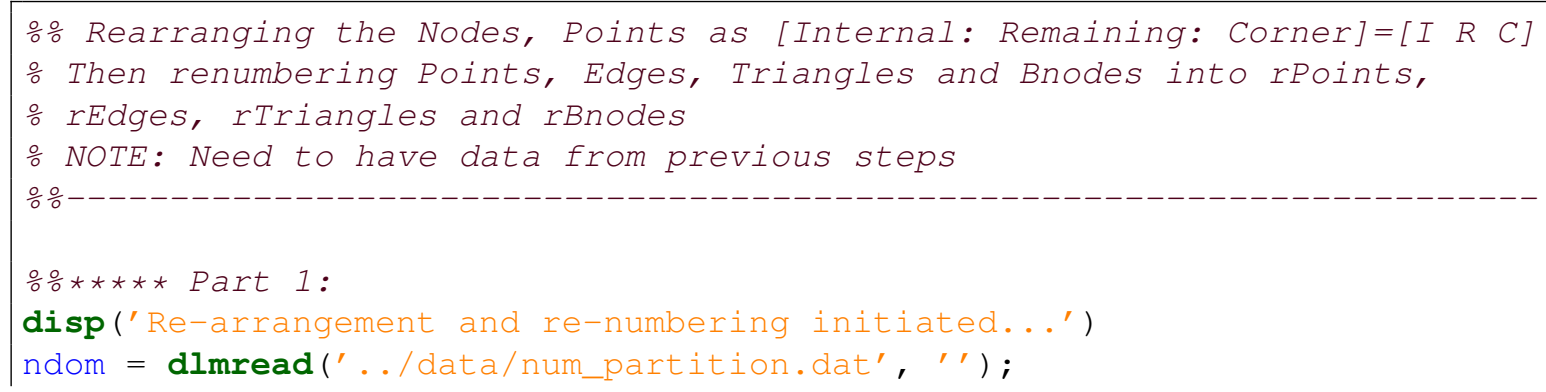




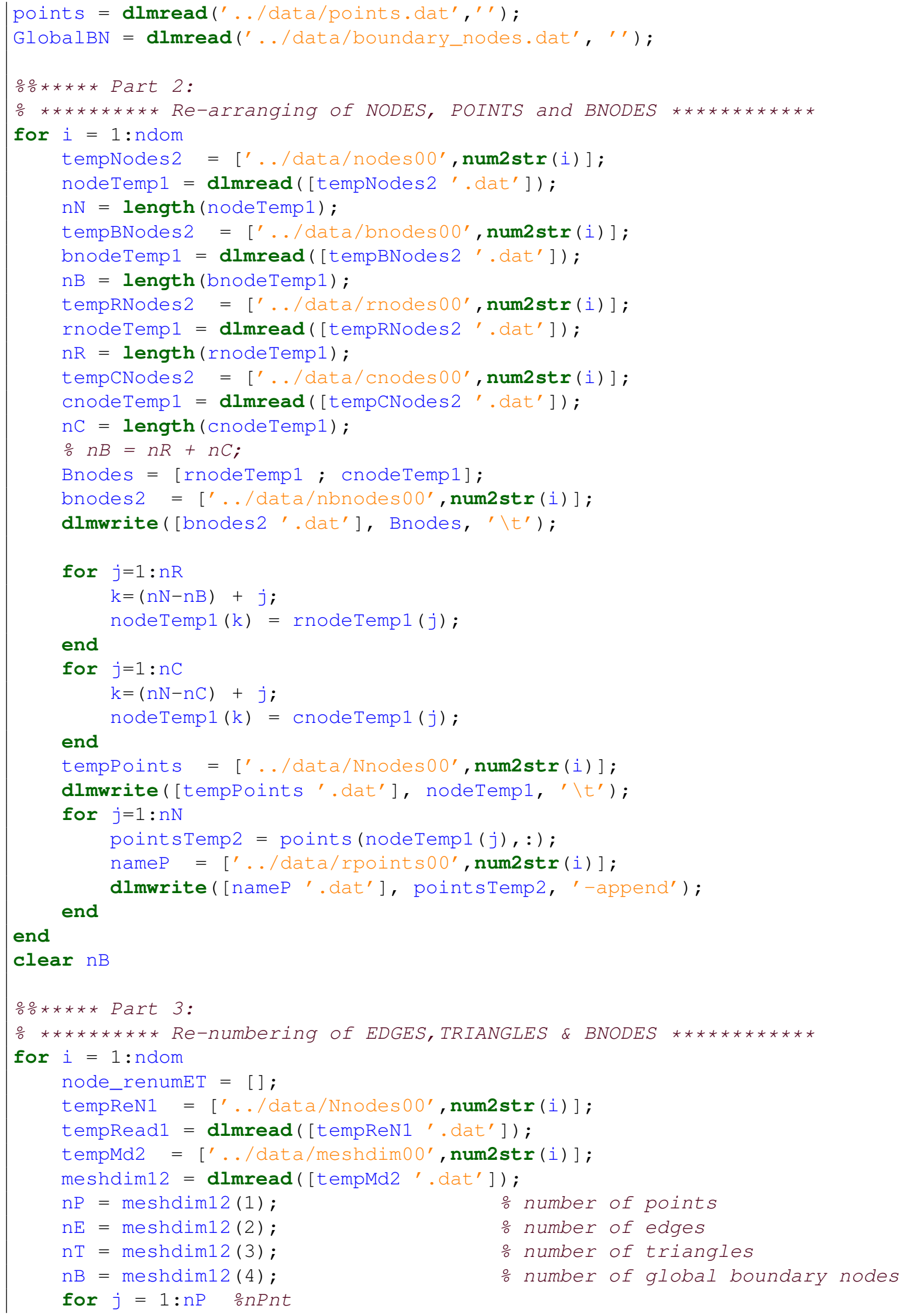




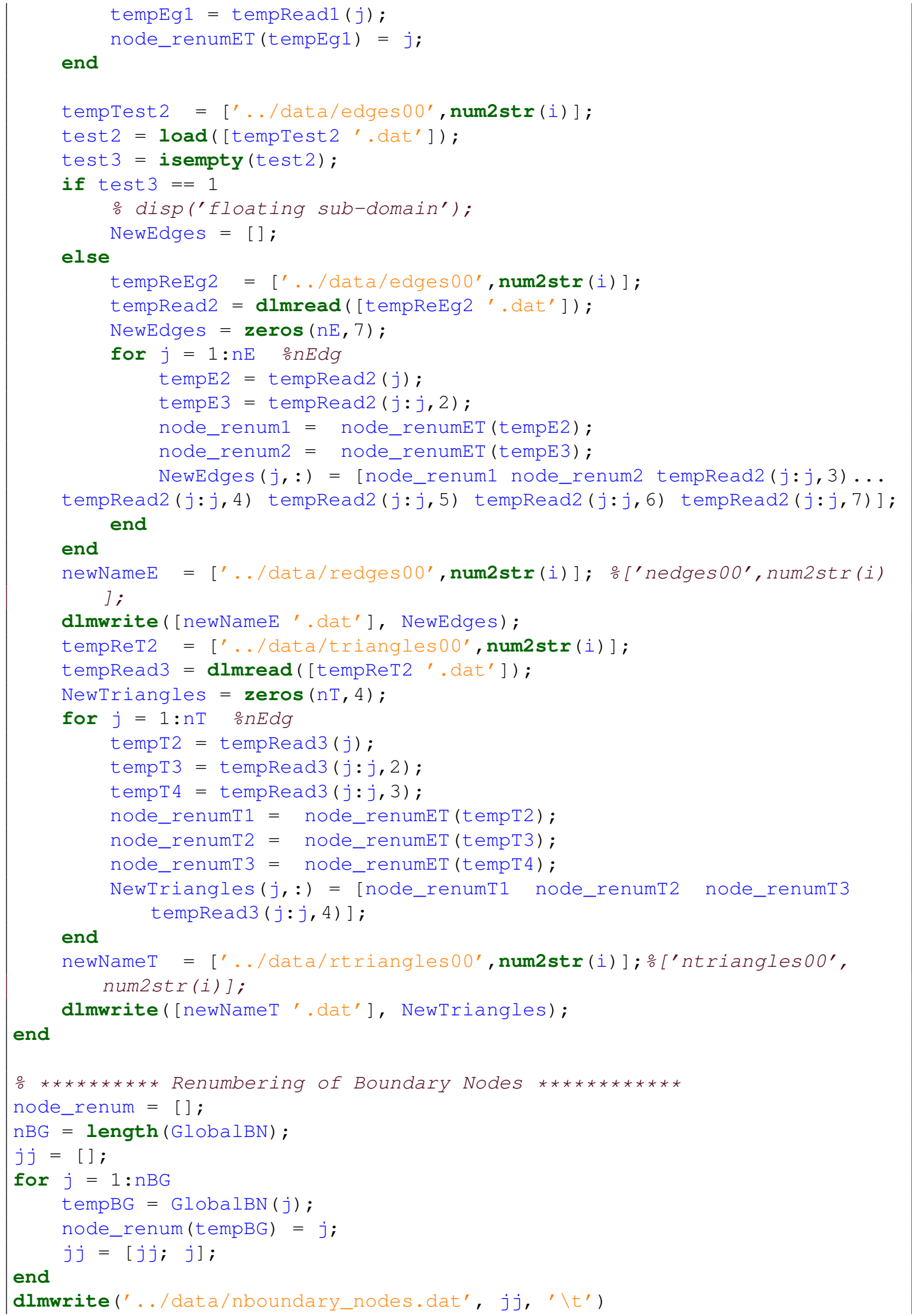




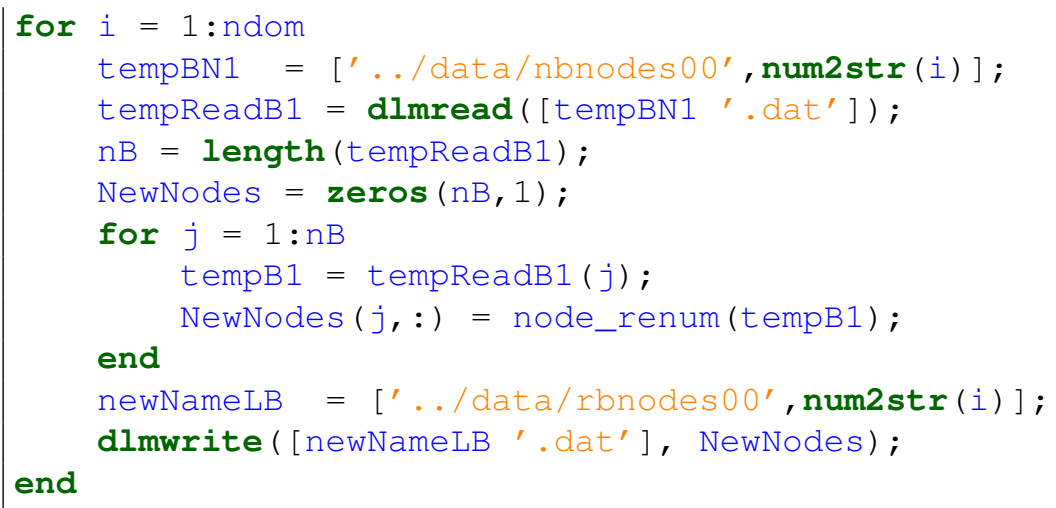

nodes0001.dat

nodes0002.dat

nodes 0003 .dat

nodes0004.dat

num_partition.dat

points.dat

points0001.dat

points0002.dat

points0003.dat

points0004.dat

remaining_nodes.dat

edges.dat
edges $0001 . d a t$
edges $0002 . d a t$
edges $0003 . d a t$
edges $0004 . d a t$
nbgc.dat
nbgr.dat
nbnodes $0001 . d a t$
nbnodes $0002 . d a t$
nbnodes $0003 . d a t$
nbnodes $0004 . d a t$

bnodes 0001. dat
bnodes $0002 . d a t$
bnodes $0003 . d a t$
bnodes $0004 . d a t$
boundary_nodes.dat
cnodes $0001 . d a t$
cnodes $0002 . d a t$
cnodes $0003 . d a t$
cnodes0004.dat
corner_nodes.dat

bnodes0001.dat rnodes0001.dat rnodes0002.dat rnodes 0003 .dat rnodes0004.dat triangles_on_boundary.dat triangles.dat triangles0001.dat triangles0002.dat triangles0003.dat triangles0004.dat

Figure D.5: A typical mesh data files extracted from the mesh file.

Listing D.8: Python code snippet to convert mesh data to FEniCS-xml data.

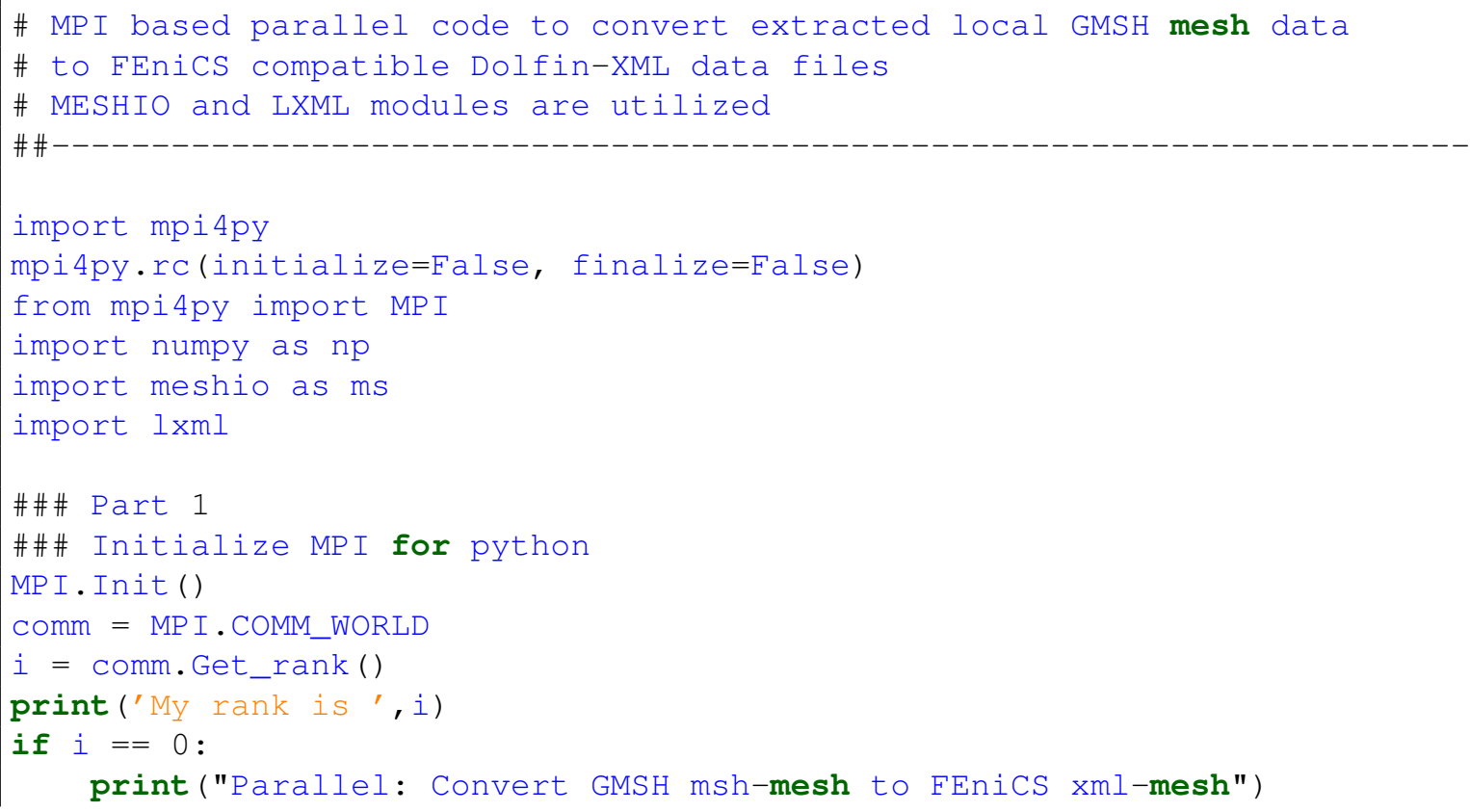




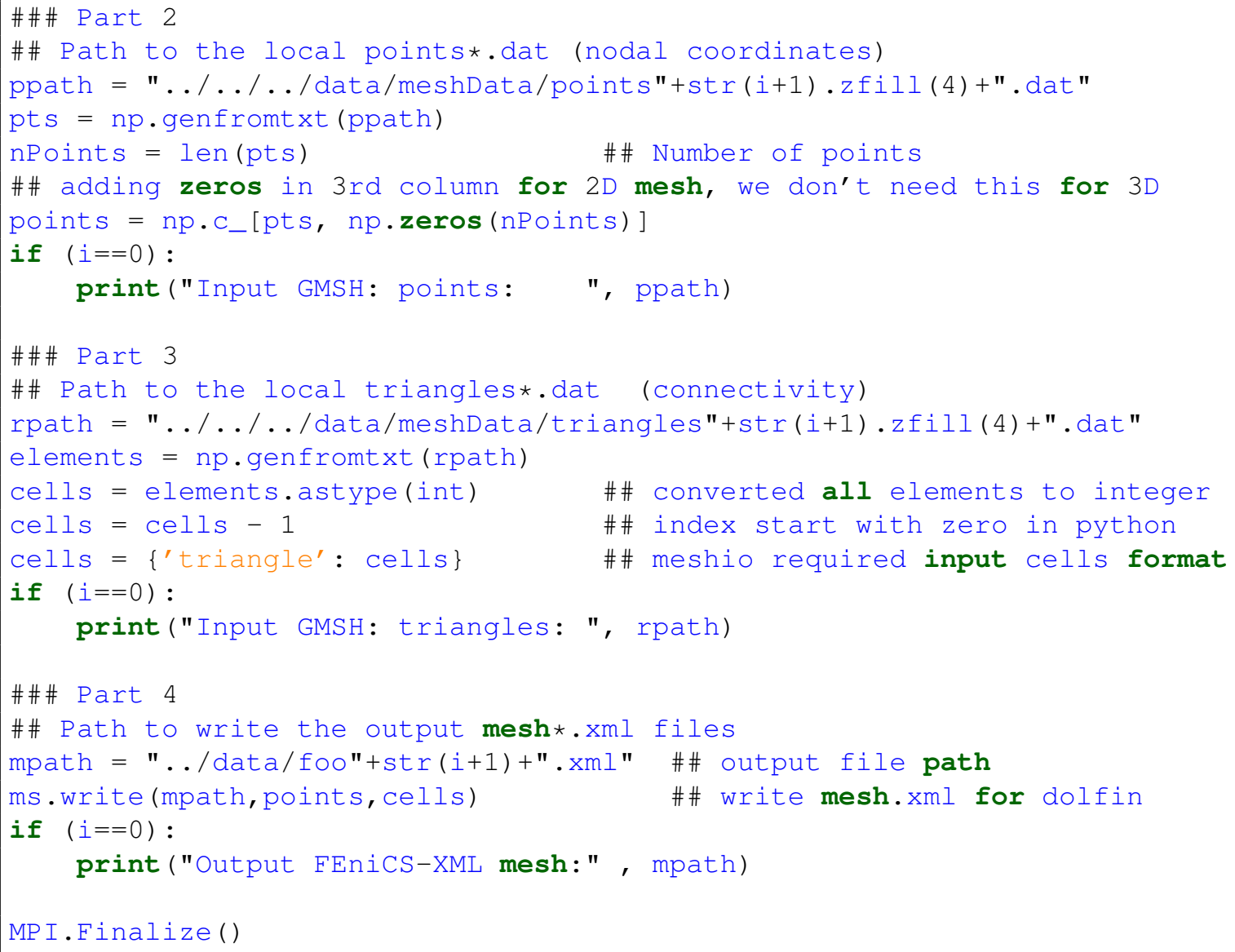

Listing D.9: FEniCS compatible XML-mesh file for a typical subdomain.

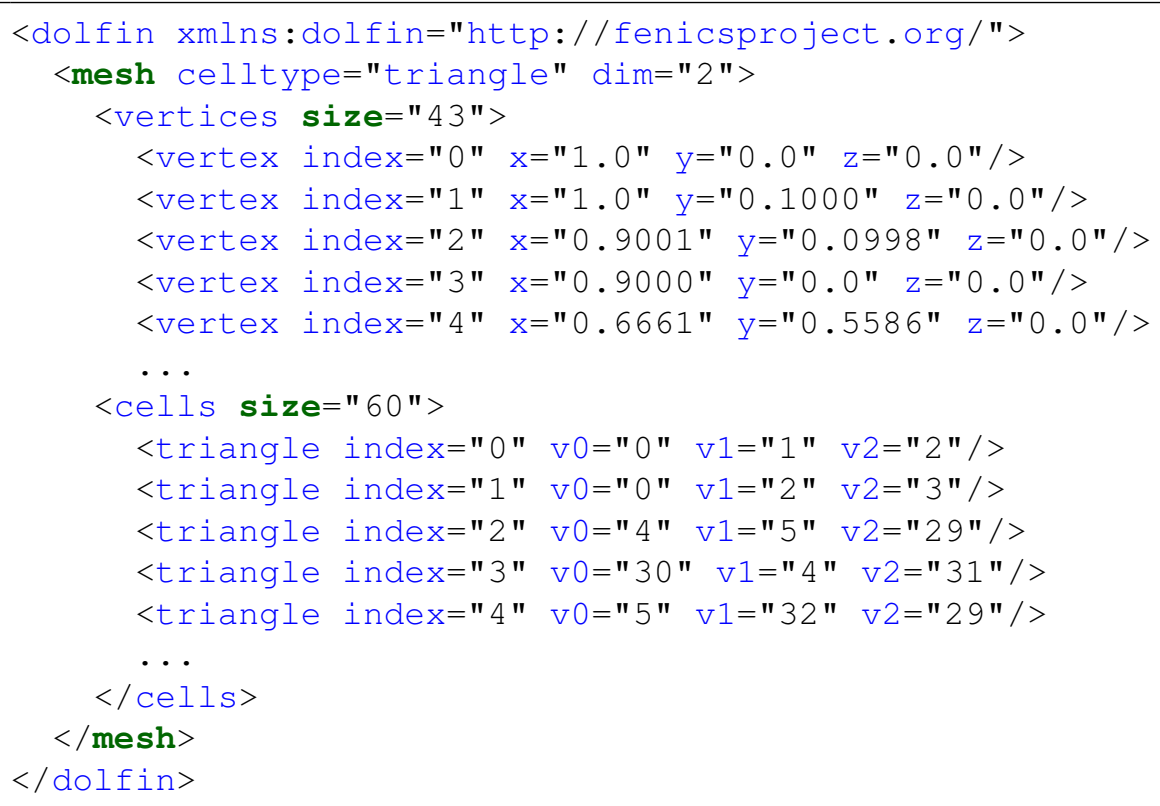




\section{Bibliography}

[1] R. Ghanem, P. Spanos, Stochastic finite elements: a spectral approach, Springer-Verlag, New York, 1991.

[2] R. Ghanem, Ingredients for a general purpose stochastic finite elements implementation, Computer Methods in Applied Mechanics and Engineering 168 (1) (1999) 19-34.

[3] U. B. Mehta, D. R. Eklund, V. J. Romero, J. A. Pearce, N. S. Keim, Simulation credibility: Advances in verification, validation, and uncertainty quantification, NASA Tech rep. URL https://ntrs.nasa.gov/archive/nasa/casi.ntrs.nasa.gov/ $20160013550 . p d f$

[4] Assessing the reliability of complex models: mathematical and statistical foundations of verification, validation, and uncertainty quantification, Tech. rep., National Research Council USA, National Academies Press (2012).

URL https://www.nap.edu/catalog/13395

[5] Supercomputer simulations have become essential to making discoveries and advancing science, Tech. rep., Lawrence Livermore National Laboratory (2005).

URL https://str.llnl.gov/str/JanFeb05/pdfs/01_05.1.pdf

[6] Simulation-based engineering science: Revolutionizing engineering science through simulation, Tech. rep., National Science Foundation, Arlington, VA (2006).

URL http://www.sci.utah.edu/publications/Ode2006a/TO_SBES_ Debrief_050306.pdf

[7] Computing 2010: from black holes to biology, Tech. rep., Nature, Vol-402 (1999).

URL http://dx.doi.org/10.1038/35011561

[8] Modeling and simulation at the exascale for energy and the environment, Tech. rep., U. S. Department of Energy Technical Report (2007).

URL http://science.energy.gov/ascr/news-and-resources/ / media/ascr/pdf/program-documents/docs/Townhall.pdf

[9] O. Le Maître, O. M. Knio, Spectral methods for uncertainty quantification: with applications to computational fluid dynamics, Springer Science \& Business Media, 2010.

[10] R. Ghanem, R. Kruger, Numerical solution of spectral stochastic finite element systems, Computer Methods in Applied Mechanics and Engineering 129 (3) (1996) 289-303. 
[11] N. Wiener, The homogeneous chaos, American Journal of Mathematics 60 (4) (1938) 897-936.

[12] R. C. Smith, Uncertainty quantification: theory, implementation, and applications, Vol. 12, SIAM, 2013.

[13] O. Knio, O. Le Maître, Uncertainty propagation in CFD using polynomial chaos decomposition, Fluid Dynamics Research 38 (9) (2006) 616-640.

[14] M. Reagan, H. Najm, B. Debusschere, O. Le Maître, O. Knio, R. Ghanem, Spectral stochastic uncertainty quantification in chemical systems, Combustion Theory and Modelling 8 (3) (2004) 607-632.

[15] C. L. Pettit, P. S. Beran, Polynomial chaos expansion applied to airfoil limit cycle oscillations, in: Proceedings of the 45th AIAA/ASME/ASCE/AHS/ASC Structures, Structural Dynamics and Materials Conference, Vol. 3, 2004, pp. 1975-1985.

[16] H. Elman, O. Ernst, D. O’Leary, M. Stewart, Efficient iterative algorithms for the stochastic finite element method with application to acoustic scattering, Computer Methods in Applied Mechanics and Engineering 194 (9-11) (2005) 1037-1055.

[17] W. Subber, A. Sarkar, A domain decomposition method of stochastic PDEs: An iterative solution techniques using a two-level scalable preconditioner, Journal of Computational Physics 257 (2014) 298-317.

[18] W. Subber, A. Sarkar, Dual-primal domain decomposition method for uncertainty quantification, Computer Methods in Applied Mechanics and Engineering 266 (2013) 112 124.

[19] A. Desai, M. Khalil, C. Pettit, D. Poirel, A. Sarkar, Scalable domain decomposition solvers for stochastic PDEs in high performance computing, Computer Methods in Applied Mechanics and Engineering 335 (2017) 194-222.

[20] G. Saad, R. Ghanem, Characterization of reservoir simulation models using a polynomial chaos-based ensemble Kalman filter, Water Resources Research 45 (4).

[21] M. Khalil, A. Sarkar, Data assimilation for large-scale computational models, in: Proceedings of the 54th AIAA/ASME/ASCE/AHS/ASC Struc tures, Structural Dynamics and Materials Conference, 2013.

[22] B. Sudret, Global sensitivity analysis using polynomial chaos expansions, Reliability Engineering \& System Safety 93 (7) (2008) 964-979.

[23] A. Sarkar, N. Benabbou, R. Ghanem, Domain decomposition of stochastic PDEs: theoretical formulations, International Journal for Numerical Methods in Engineering 77 (5) (2009) 689-701.

[24] W. Subber, Domain decomposition methods for uncertainty quantification, Ph.D. thesis, Carleton University Ottawa (2012). 
[25] J. H. Bramble, J. E. Pasciak, A. H. Schatz, The construction of preconditioners for elliptic problems by substructuring. iv, Mathematics of Computation 53 (187) (1989) 1-24.

[26] B. F. Smith, A domain decomposition algorithm for elliptic problems in three dimensions, Numerische Mathematik 60 (1) (1991) 219-234.

[27] GMSH- a three-dimensional finite element mesh generator (2017).

URL http://gmsh.info/doc/texinfo/gmsh.html

[28] METIS- serial graph partitioning (2017).

URL http://glaros.dtc.umn.edu/gkhome/views/metis

[29] B. Debusschere, K. Sargsyan, C. Safta, UQTk version 2.1 user manual, Tech. rep., Sandia National Laboratory (SNL) (2013).

URL http: //wWw.sandia.gov/UQToolkit/

[30] Meshio: Python package to convert between various unstructured mesh formats (2017). URL https://pypi.org/project/meshio/

[31] S. Balay, S. Abhyankar, M. Adams, J. Brown, P. Brune, K. Buschelman, L. Dalcin, V. Eijkhout, W. Gropp, D. Kaushik, M. Knepley, D. May, L. McInnes, K. Rupp, P. Sanan, B. Smith, PETSc users manual 3.7, Tech. rep., Argonne National Laboratory (2017). URL http://www.mcs.anl.gov/petsc

[32] A. Logg, A. Mardal, G. Wells, Automated solution of differential equations by the finite element method: The FEniCS book, Vol. 84, Springer Science \& Business Media, 2012. URL http: / / fenicsproject.org

[33] W. Gropp, E. Lusk, A. Skjellum, Using MPI: portable parallel programming with the message-passing interface, Vol. 1, MIT press, 1999.

[34] ParaView- multi-platform data analysis and visualization application (2017).

URL https: //www.paraview.org

[35] The Mathworks Inc: Matlab user's guide (2015).

URL https://www. mathworks.com

[36] Scientific impacts and opportunities for computing, Tech. rep., Workshop Report by U.S. Department of Energy and Advanced Scientific Computing Research (2008).

URL http://computing.ornl.gov/workshops/peta08/

[37] Supercomputer simulates nuclear explosion down to the molecular level, Tech. rep., National Nuclear Security Administration (2012).

URL http://eng.spb-venchur.ru/news/16292.htm

[38] NASA experts share importance of supercomputing, Tech. rep., National Aeronautics and Space Administration (2015).

URL http://www.nasa.gov/high-tech-computing/index.html 
[39] R. L. Iman, J. C. Helton, An investigation of uncertainty and sensitivity analysis techniques for computer models, Risk Analysis 8 (1) (1988) 71-90.

[40] M. D. McKay, R. J. Beckman, W. J. Conover, A comparison of three methods for selecting values of input variables in the analysis of output from a computer code, Technometrics 42 (1) (2000) 55-61.

[41] B. M. Ayyub, Elicitation of expert opinions for uncertainty and risks, CRC press, 2001.

[42] W. L. Oberkampf, S. M. DeLand, B. M. Rutherford, K. V. Diegert, K. F. Alvin, Error and uncertainty in modeling and simulation, Reliability Engineering \& System Safety 75 (3) (2002) 333-357.

[43] H. G. Matthies, Quantifying uncertainty: modern computational representation of probability and applications, in: Extreme man-made and natural hazards in dynamics of structures, Springer, 2007, pp. 105-135.

[44] R. Ghanem, Uncertainty quantification in computational and prediction science, International Journal for Numerical Methods in Engineering 80 (6-7) (2009) 671-672.

[45] W. L. Oberkampf, T. G. Trucano, C. Hirsch, Verification, validation, and predictive capability in computational engineering and physics, Applied Mechanics Reviews 57 (5) (2004) 345-384.

[46] R. G. Sargent, Verification and validation of simulation models, in: Proceedings of the 37th Conference on Winter Simulation, 2005, pp. 130-143.

[47] W. L. Oberkampf, C. J. Roy, Verification and validation in scientific computing, Cambridge University Press, 2010.

[48] B. M. Ayyub, G. J. Klir, Uncertainty modeling and analysis in engineering and the sciences, CRC Press, 2006.

[49] C. J. Roy, W. L. Oberkampf, A comprehensive framework for verification, validation, and uncertainty quantification in scientific computing, Computer Methods in Applied Mechanics and Engineering 200 (25) (2011) 2131-2144.

[50] J. Sacks, W. J. Welch, T. J. Mitchell, H. P. Wynn, Design and analysis of computer experiments, Statistical Science (1989) 409-423.

[51] R. Jain, The art of computer systems performance analysis: techniques for experimental design, measurement, simulation, and modeling, John Wiley \& Sons, 1990.

[52] W. L. Oberkampf, S. M. DeLand, B. M. Rutherford, K. V. Diegert, K. F. Alvin, Estimation of total uncertainty in modeling and simulation, Sandia Report SAND2000-0824, Albuquerque, NM.

[53] G. Iaccarino, Quantification of uncertainty in flow simulations using probabilistic methods, Tech. rep., DTIC Document (2009). 
[54] AIAA guide for the verification and validation of computational fluid dynamics simulations (G-077-1998) (1998).

[55] W. E. Walker, P. Harremoës, J. Rotmans, J. P. van der Sluijs, M. B. van Asselt, P. Janssen, M. P. Krayer von Krauss, Defining uncertainty: a conceptual basis for uncertainty management in model-based decision support, Integrated Assessment 4 (1) (2003) 5-17.

[56] D. Lucor, D. Xiu, C. Su, G. Karniadakis, Predictability and uncertainty in CFD, International Journal for Numerical Methods in Fluids 43 (5) (2003) 483-505.

[57] J. Faragher, Probabilistic methods for the quantification of uncertainty and error in computational fluid dynamic simulations, Tech. rep., DTIC Document (2004).

[58] A. Der Kiureghian, O. Ditlevsen, Aleatory or epistemic? does it matter?, Structural Safety 31 (2) (2009) 105-112.

[59] M. P. Pettersson, G. Iaccarino, J. Nordstrom, Polynomial chaos methods for hyperbolic partial differential equations, Springer Math Eng. doi 10 (1007) (2015) 978-3.

[60] P. S. Beran, C. L. Pettit, D. R. Millman, Uncertainty quantification of limit-cycle oscillations, Journal of Computational Physics 217 (1) (2006) 217-247.

[61] I. Babuska, R. Tempone, G. E. Zouraris, Galerkin finite element approximations of stochastic elliptic partial differential equations, SIAM Journal on Numerical Analysis 42 (2) (2004) 800-825.

[62] B. Ganapathysubramanian, N. Zabaras, Sparse grid collocation schemes for stochastic natural convection problems, Journal of Computational Physics 225 (1) (2007) 652-685.

[63] A. Nouy, Recent developments in spectral stochastic methods for the numerical solution of stochastic partial differential equations, Archives of Computational Methods in Engineering 16 (3) (2009) 251-285.

[64] D. Xiu, Numerical methods for stochastic computations: a spectral method approach, Princeton University Press, 2010.

[65] N. Metropolis, S. Ulam, The Monte Carlo method, Journal of the American Statistical Association 44 (247) (1949) 335-341.

[66] S. M. Stigler, The history of statistics: The measurement of uncertainty before 1900, Harvard University Press, 1986.

[67] M. Papadrakakis, V. Papadopoulos, Robust and efficient methods for stochastic finite element analysis using Monte Carlo simulation, Computer Methods in Applied Mechanics and Engineering 134 (3) (1996) 325-340.

[68] M. Kleiber, T. D. Hien, The stochastic finite element method: basic perturbation technique and computer implementation, Wiley, 1992. 
[69] R. Ghosh, S. Chakraborty, B. Bhattacharyya, Stochastic sensitivity analysis of structures using first-order perturbation, Meccanica 36 (3) (2001) 291-296.

[70] I. Babuška, P. Chatzipantelidis, On solving elliptic stochastic partial differential equations, Computer Methods in Applied Mechanics and Engineering 191 (37) (2002) 4093 4122 .

[71] D. Xiu, G. Karniadakis, The Wiener-Askey polynomial chaos for stochastic differential equations, SIAM Journal on Scientific Computing 24 (2) (2002) 619-644.

[72] G. Stefanou, The stochastic finite element method: past, present and future, Computer Methods in Applied Mechanics and Engineering 198 (9) (2009) 1031-1051.

[73] P. G. Ciarlet, The finite element method for elliptic problems, SIAM, 2002.

[74] W. K. Liu, T. Belytschko, A. Mani, Random field finite elements, International Journal for Numerical Methods in Engineering 23 (10) (1986) 1831-1845.

[75] A. Der Kiureghian, J. Ke, The stochastic finite element method in structural reliability, Probabilistic Engineering Mechanics 3 (2) (1988) 83-91.

[76] I. Babuška, R. Tempone, G. E. Zouraris, Solving elliptic boundary value problems with uncertain coefficients by the finite element method: the stochastic formulation, Computer Methods in Applied Mechanics and Engineering 194 (12) (2005) 1251-1294.

[77] K. Binder, Introduction: theory and "technical" aspects of Monte Carlo simulations, in: Monte Carlo Methods in Statistical Physics, Springer, 1986, pp. 1-45.

[78] D. Xiu, J. S. Hesthaven, High-order collocation methods for differential equations with random inputs, SIAM Journal on Scientific Computing 27 (3) (2005) 1118-1139.

[79] S. Hosder, R. W. Walters, R. Perez, A non-intrusive polynomial chaos method for uncertainty propagation in CFD simulations, AIAA Paper 891 (2006) 2006.

[80] I. Babuška, F. Nobile, R. Tempone, A stochastic collocation method for elliptic partial differential equations with random input data, SIAM Journal on Numerical Analysis 45 (3) (2007) 1005-1034.

[81] G. Loeven, J. Witteveen, H. Bijl, Probabilistic collocation: an efficient non-intrusive approach for arbitrarily distributed parametric uncertainties, in: Proceedings of the 45th AIAA Aerospace Sciences Meeting and Exhibit, AIAA paper, Vol. 317, 2007.

[82] K. A. Cliffe, M. B. Giles, R. Scheichl, A. L. Teckentrup, Multilevel Monte Carlo methods and applications to elliptic PDEs with random coefficients, Computing and Visualization in Science 14 (1) (2011) 3-15.

[83] D. Xiu, G. Karniadakis, Modeling uncertainty in steady state diffusion problems via generalized polynomial chaos, Computer Methods in Applied Mechanics and Engineering 191 (43) (2002) 4927-4948. 
[84] P. B. Nair, A. J. Keane, Stochastic reduced basis methods, AIAA journal 40 (8) (2002) 1653-1664.

[85] O. Le Mattre, O. Knio, H. Najm, R. Ghanem, Uncertainty propagation using WienerHaar expansions, Journal of Computational Physics 197 (1) (2004) 28-57.

[86] H. G. Matthies, A. Keese, Galerkin methods for linear and nonlinear elliptic stochastic partial differential equations, Computer Methods in Applied Mechanics and Engineering 194 (12) (2005) 1295-1331.

[87] D. P. Landau, K. Binder, A guide to Monte Carlo simulations in statistical physics, Cambridge University Press, 2014.

[88] O. Knio, H. Najm, R. Ghanem, et al., A stochastic projection method for fluid flow: I. basic formulation, Journal of Computational Physics 173 (2) (2001) 481-511.

[89] X. Wan, D. Xiu, G. Karniadakis, Stochastic solutions for the two-dimensional advectiondiffusion equation, SIAM Journal on Scientific computing 26 (2) (2004) 578-590.

[90] M. Pellissetti, R. Ghanem, Iterative solution of systems of linear equations arising in the context of stochastic finite elements, Advances in Engineering Software 31 (8) (2000) 607-616.

[91] M. Schevenels, G. Lombaert, G. Degrande, Application of the stochastic finite element method for Gaussian and non-Gaussian systems, in: ISMA2004 International Conference on Noise and Vibration Engineering, 2004, pp. 3299-3314.

[92] P. Dostert, Y. Efendiev, T. Y. Hou, Multiscale finite element methods for stochastic porous media flow equations and application to uncertainty quantification, Computer Methods in Applied Mechanics and Engineering 197 (43) (2008) 3445-3455.

[93] O. Le Maître, M. Reagan, H. Najm, R. Ghanem, O. Knio, A stochastic projection method for fluid flow: II. random process, Journal of Computational Physics 181 (1) (2002) 9-44.

[94] J. A. Witteveen, Efficient and robust uncertainty quantification for computational fluid dynamics and fluid-structure interaction, TU Delft, Delft University of Technology, 2009.

[95] P. J. Roache, Quantification of uncertainty in computational fluid dynamics, Annual Review of Fluid Mechanics 29 (1) (1997) 123-160.

[96] H. N. Najm, Uncertainty quantification and polynomial chaos techniques in computational fluid dynamics, Annual Review of Fluid Mechanics 41 (2009) 35-52.

[97] G. J. Loeven, Efficient uncertainty quantification in computational fluid dynamics, TU Delft, Delft University of Technology, 2010.

[98] D. Xiu, D. Lucor, C. Su, G. Karniadakis, Stochastic modeling of flow-structure interactions using generalized polynomial chaos, Journal of Fluids Engineering 124 (1) (2002) $51-59$. 
[99] J. A. Witteveen, S. Sarkar, H. Bijl, Modeling physical uncertainties in dynamic stall induced fluid-structure interaction of turbine blades using arbitrary polynomial chaos, Computers \& structures 85 (11) (2007) 866-878.

[100] J. A. Witteveen, H. Bijl, Higher period stochastic bifurcation of nonlinear airfoil fluidstructure interaction, Mathematical Problems in Engineering 2009.

[101] A. Desai, S. Sarkar, Analysis of a nonlinear aeroelastic system with parametric uncertainties using polynomial chaos expansion, Mathematical Problems in Engineering 2010.

[102] C. L. Pettit, Uncertainty quantification in aeroelasticity: recent results and research challenges, Journal of Aircraft 41 (5) (2004) 1217-1229.

[103] S. Sarkar, J. Witteveen, G. J. Loeven, H. Bijl, Effect of uncertainty on the bifurcation behavior of pitching airfoil stall flutter, Journal of Fluids and Structures 25 (2) (2009) 304-320.

[104] A. Desai, S. Sarkar, Uncertainty quantification and bifurcation behavior of an aeroelastic system, in: ASME 2010 3rd Joint US-European Fluids Engineering Summer Meeting, American Society of Mechanical Engineers, 2010, pp. 1177-1187.

[105] A. Desai, J. A. S. Witteveen, S. Sarkar, Uncertainty quantification of a nonlinear aeroelastic system using polynomial chaos expansion with constant phase interpolation, Journal of Vibration and Acoustics 135 (5) (2013) 051034.

[106] M. Eldred, J. Burkardt, Comparison of non-intrusive polynomial chaos and stochastic collocation methods for uncertainty quantification, AIAA paper 976 (2009) 1-20.

[107] B. J. Debusschere, H. N. Najm, P. P. Pébay, O. M. Knio, R. G. Ghanem, O. P. Le Maître, Numerical challenges in the use of polynomial chaos representations for stochastic processes, SIAM Journal on Scientific Computing 26 (2) (2004) 698-719.

[108] M. Reagana, H. Najm, R. Ghanem, O. Knio, Uncertainty quantification in reacting-flow simulations through non-intrusive spectral projection, Combustion and Flame 132 (3) (2003) 545-555.

[109] D. Wei, Z. Cui, J. Chen, Uncertainty quantification using polynomial chaos expansion with points of monomial cubature rules, Computers \& Structures 86 (23) (2008) 21022108.

[110] D. Xiu, Efficient collocational approach for parametric uncertainty analysis, Commun. Comput. Phys 2 (2) (2007) 293-309.

[111] F. Nobile, R. Tempone, C. G. Webster, A sparse grid stochastic collocation method for partial differential equations with random input data, SIAM Journal on Numerical Analysis 46 (5) (2008) 2309-2345.

[112] L. Mathelin, M. Y. Hussaini, T. A. Zang, Stochastic approaches to uncertainty quantification in CFD simulations, Numerical Algorithms 38 (1-3) (2005) 209-236. 
[113] H. C. Elman, C. W. Miller, E. T. Phipps, R. S. Tuminaro, Assessment of collocation and Galerkin approaches to linear diffusion equations with random data, International Journal for Uncertainty Quantification 1 (2011) 1.

[114] X. Ma, N. Zabaras, An adaptive hierarchical sparse grid collocation algorithm for the solution of stochastic differential equations, Journal of Computational Physics 228 (8) (2009) 3084-3113.

[115] D. Xiu, Fast numerical methods for stochastic computations: a review, Communications in Computational Physics 5 (2-4) (2009) 242-272.

[116] J. Bäck, F. Nobile, L. Tamellini, R. Tempone, Stochastic spectral Galerkin and collocation methods for PDEs with random coefficients: a numerical comparison, in: Spectral and High Order Methods for Partial Differential Equations, Springer, 2011, pp. 43-62.

[117] S. K. Sachdeva, P. B. Nair, A. J. Keane, Comparative study of projection schemes for stochastic finite element analysis, Computer Methods in Applied Mechanics and Engineering 195 (19) (2006) 2371-2392.

[118] R. Tipireddy, E. Phipps, R. Ghanem, A comparison of solution methods for stochastic partial differential equations, CSRI Summer Proceedings 201079.

[119] L. Giraldi, A. Litvinenko, D. Liu, H. G. Matthies, A. Nouy, To be or not to be intrusive? the solution of parametric and stochastic equations-the "plain vanilla" Galerkin case, SIAM Journal on Scientific Computing 36 (6) (2014) A2720-A2744.

[120] M. Eiermann, O. G. Ernst, E. Ullmann, Computational aspects of the stochastic finite element method, Computing and Visualization in Science 10 (1) (2007) 3-15.

[121] D. E. Keyes, W. D. Gropp, A comparison of domain decomposition techniques for elliptic partial differential equations and their parallel implementation, SIAM Journal on Scientific and Statistical Computing 8 (2) (1987) s166-s202.

[122] G. Yagawa, N. Soneda, S. Yoshimura, A large scale finite element analysis using domain decomposition method on a parallel computer, Computers \& Structures 38 (5-6) (1991) $615-625$.

[123] J. Mandel, Balancing domain decomposition, Communications in Numerical Methods in Engineering 9 (3) (1993) 233-241.

[124] T. F. Chan, T. P. Mathew, Domain decomposition algorithms, Acta numerica 3 (1994) 61-143.

[125] A. Quarteroni, A. Valli, Domain decomposition methods for partial differential equations, Oxford University Press, 1999.

[126] E. Faccioli, F. Maggio, A. Quarteroni, A. Taghan, Spectral-domain decomposition methods for the solution of acoustic and elastic wave equations, Geophysics 61 (4) (1996) 1160-1174. 
[127] C. Jin, X. Cai, C. Li, Parallel domain decomposition methods for stochastic elliptic equations, SIAM Journal on Scientific Computing 29 (5) (2007) 2096-2114.

[128] A. Toselli, O. Widlund, Domain decomposition methods: algorithms and theory, Vol. 34, Springer, 2005.

[129] W. D. Gropp, Parallel computing and domain decomposition, in: Fifth International Symposium on Domain Decomposition Methods for Partial Differential Equations, Philadelphia, PA, 1992.

[130] J. Kruis, Domain decomposition methods for distributed computing, Saxe-Coburg Publications, 2007.

[131] C. Farhat, M. Lesoinne, K. Pierson, A scalable dual-primal domain decomposition method, Numerical Linear Algebra with Applications 7 (7-8) (2000) 687-714.

[132] Y. Fragakis, M. Papadrakakis, The mosaic of high performance domain decomposition methods for structural mechanics: Formulation, interrelation and numerical efficiency of primal and dual methods, Computer Methods in Applied Mechanics and Engineering 192 (35) (2003) 3799-3830.

[133] P. Gosselet, C. Rey, Non-overlapping domain decomposition methods in structural mechanics, Archives of Computational Methods in Engineering 13 (4) (2006) 515-572.

[134] P. Le Tallec, J. Mandel, M. Vidrascu, A Neumann-Neumann domain decomposition algorithm for solving plate and shell problems, SIAM Journal on Numerical Analysis 35 (2) (1998) 836-867.

[135] T. F. Chan, Domain decomposition algorithms and computational fluid dynamics, The International Journal of Supercomputing Applications 2 (4) (1988) 72-83.

[136] W. D. Gropp, D. E. Keyes, Domain decomposition methods in computational fluid dynamics, International journal for numerical methods in fluids 14 (2) (1992) 147-165.

[137] P. F. Fischer, An overlapping Schwarz method for spectral element solution of the incompressible Navier-Stokes equations, Journal of Computational Physics 133 (1) (1997) 84-101.

[138] C. Farhat, P. Avery, R. Tezaur, J. Li, FETI-DPH: a dual-primal domain decomposition method for acoustic scattering, Journal of Computational Acoustics 13 (2005) 499-524.

[139] B. Smith, P. Bjorstad, W. Gropp, Domain decomposition: parallel multilevel methods for elliptic partial differential equations, Cambridge University Press, 2004.

[140] J. Cros, A preconditioner for the Schur complement domain decomposition method, Domain decomposition methods in science and engineering (2003) 373-380.

[141] J. Mandel, C. R. Dohrmann, R. Tezaur, An algebraic theory for primal and dual substructuring methods by constraints, Applied Numerical Mathematics 54 (2) (2005) 167-193. 
[142] M. Papadrakakis, G. Stavroulakis, A. Karatarakis, A new era in scientific computing: Domain decomposition methods in hybrid CPU-GPU architectures, Computer Methods in Applied Mechanics and Engineering 200 (13) (2011) 1490-1508.

[143] E. Ullmann, H. C. Elman, O. G. Ernst, Efficient iterative solvers for stochastic Galerkin discretizations of log-transformed random diffusion problems, SIAM Journal on Scientific Computing 34 (2) (2012) A659-A682.

[144] J. Mandel, C. R. Dohrmann, Convergence of a balancing domain decomposition by constraints and energy minimization, Numerical Linear Algebra with Applications 10 (7) (2003) 639-659.

[145] J. Mandel, B. Sousedík, C. R. Dohrmann, Multispace and multilevel BDDC, Computing $83(2-3)(2008)$ 55-85.

[146] C. Farhat, F.-X. Roux, A method of finite element tearing and interconnecting and its parallel solution algorithm, International Journal for Numerical Methods in Engineering 32 (6) (1991) 1205-1227.

[147] C. Farhat, J. Mandel, F. X. Roux, Optimal convergence properties of the FETI domain decomposition method, Computer Methods in Applied Mechanics and Engineering 115 (34) (1994) 365-385.

[148] D. Ghosh, P. Avery, C. Farhat, A FETI-preconditioned conjugate gradient method for large-scale stochastic finite element problems, International Journal for Numerical Methods in Engineering 80 (6-7) (2009) 914-931.

[149] J. Li, O. B. Widlund, FETI-DP, BDDC, and block Cholesky methods, International journal for numerical methods in engineering 66 (2) (2006) 250-271.

[150] Y. Saad, Iterative methods for sparse linear systems, SIAM, 2003.

[151] E. Rosseel, S. Vandewalle, Iterative solvers for the stochastic finite element method, SIAM Journal on Scientific Computing 32 (1) (2010) 372-397.

[152] H. Elman, D. Furnival, Solving the stochastic steady-state diffusion problem using multigrid, IMA Journal of Numerical Analysis 27 (4) (2007) 675-688.

[153] C. E. Powell, H. C. Elman, Block-diagonal preconditioning for spectral stochastic finiteelement systems, IMA Journal of Numerical Analysis 29 (2) (2009) 350-375.

[154] E. Ullmann, A Kronecker product preconditioner for stochastic Galerkin finite element discretization, SIAM Journal on Scientific Computing 32 (2) (2010) 923-946.

[155] B. Sousedík, R. Ghanem, E. Phipps, Hierarchical Schur complement preconditioner for the stochastic Galerkin finite element methods, Numerical Linear Algebra with Applications 21 (1) (2014) 136-151.

[156] W. Subber, S. Loisel, Schwarz preconditioners for stochastic elliptic PDEs, Computer Methods in Applied Mechanics and Engineering 272 (2014) 34-57. 
[157] N. Spillane, An adaptive multipreconditioned conjugate gradient algorithm, SIAM journal on Scientific Computing 38 (3) (2016) A1896-A1918.

[158] S. Albers, Energy-efficient algorithms, Communications of the ACM 53 (2010) 86-96.

[159] M. Hoemmen, Communication-avoiding Krylov subspace methods, Ph.D. thesis, University of California, Berkeley (2010).

[160] The future of supercomputing-an interim report, Tech. rep., National Research Council of the National Academies (2003).

URL http: / /www.nap.edu/catalog/10784.html

[161] E. Phipps, H. C. Edwards, J. Hu, J. T. Ostien, Exploring emerging manycore architectures for uncertainty quantification through embedded stochastic Galerkin methods, International Journal of Computer Mathematics 91 (4) (2014) 707-729.

[162] M. S. Eldred, Recent advances in non-intrusive polynomial chaos and stochastic collocation methods for uncertainty analysis and design, AIAA Paper 2274 (2009) 37.

[163] H. N. Najm, Quantification of uncertainty in extreme scale computations, in: 2014 SciDAC-3 PI Meeting, Washington, DC, 2014.

[164] K. Bergman, S. Borkar, D. Campbell, W. Carlson, W. Dally, M. Denneau, P. Franzon, W. Harrod, K. Hill, J. Hiller, et al., Exascale computing study: Technology challenges in achieving exascale systems, Defense Advanced Research Projects Agency Information Processing Techniques Office (DARPA IPTO), Tech. Rep 15.

[165] S. Ashby, P. Beckman, J. Chen, P. Colella, B. Collins, D. Crawford, J. Dongarra, D. Kothe, R. Lusk, P. Messina, et al., The opportunities and challenges of exascale computing, Summary Report of the Advanced Scientific Computing Advisory Committee (ASCAC) Subcommittee (2010) 1-77.

[166] I. S. Duff, European exascale software initiative: Numerical libraries, solvers and algorithms, in: European Conference on Parallel Processing, Springer, 2011, pp. 295-304.

[167] I. S. Duff, A. M. Erisman, J. K. Reid, Direct methods for sparse matrices, Clarendon press Oxford, 1986.

[168] T. Coupez, S. Marie, From a direct solver to a parallel iterative solver in 3-D forming simulation, International Journal of High Performance Computing Applications 11 (4) (1997) 277-285.

[169] O. Le Ma1tre, O. Knio, B. Debusschere, H. Najm, R. Ghanem, A multigrid solver for twodimensional stochastic diffusion equations, Computer Methods in Applied Mechanics and Engineering 192 (41) (2003) 4723-4744.

[170] D. B. Chung, M. A. Gutiérrez, L. L. Graham-Brady, F.-J. Lingen, Efficient numerical strategies for spectral stochastic finite element models, International journal for numerical methods in engineering 64 (10) (2005) 1334-1349. 
[171] C. Jin, X. Cai, C. Li, Parallel domain decomposition methods for stochastic elliptic equations, SIAM Journal on Scientific Computing 29 (5) (2007) 2096-2114.

[172] D. Silvester, Pranjal, An optimal solver for linear systems arising from stochastic FEM approximation of diffusion equations with random coefficients, SIAM/ASA Journal on Uncertainty Quantification 4 (1) (2016) 298-311.

[173] C. E. Powell, D. Silvester, V. Simoncini, An efficient reduced basis solver for stochastic Galerkin matrix equations, SIAM Journal on Scientific Computing 39 (1) (2017) A141A163.

[174] G. Stavroulakis, D. Giovanis, V. Papadopoulos, M. Papadrakakis, A GPU domain decomposition solution for spectral stochastic finite element method, Computer Methods in Applied Mechanics and Engineering 327 (2017) 392-410.

[175] S. Pranesh, D. Ghosh, A FETI-DP based parallel hybrid stochastic finite element method for large stochastic systems, Computers \& Structures 195 (2018) 64-73.

[176] X. Ma, N. Zabaras, An adaptive high-dimensional stochastic model representation technique for the solution of stochastic partial differential equations, Journal of Computational Physics 229 (10) (2010) 3884-3915.

[177] A. Logg, G. Wells, J. Hake, DOLFIN: A C++/Python finite element library, in: Automated Solution of Differential Equations by the Finite Element Method, Springer, 2012. URL https://bitbucket.org/fenics-project/dolfin

[178] M. Khalil, Bayesian inference for complex and large-scale engineering systems, Ph.D. thesis, Carleton University Ottawa (2013).

[179] C. Canuto, M. Y. Hussaini, A. Quarteroni, A. Thomas Jr, et al., Spectral methods in fluid dynamics, Springer Science \& Business Media, 2012.

[180] A. Papoulis, S. U. Pillai, Probability, random variables, and stochastic processes, Tata McGraw-Hill Education, 2002.

[181] P. Billingsley, Probability and measure, John Wiley \& Sons, 2008.

[182] R. Ghanem, Stochastic finite elements with multiple random non-Gaussian properties, Journal of Engineering Mechanics 125 (1) (1999) 26-40.

[183] R. Ghanem, The nonlinear Gaussian spectrum of log-normal stochastic processes and variables, Journal of applied mechanics 66 (4) (1999) 964-973.

[184] M. Rosenblatt, Remarks on a multivariate transformation, The annals of mathematical statistics 23 (3) (1952) 470-472.

[185] C. Soize, R. Ghanem, Physical systems with random uncertainties: chaos representations with arbitrary probability measure, SIAM Journal on Scientific Computing 26 (2) (2004) 395-410. 
[186] S. Rahman, A polynomial chaos expansion in dependent random variables, Journal of Mathematical Analysis and Applications 464 (1) (2018) 749-775.

[187] S. Huang, S. Quek, K. Phoon, Convergence study of the truncated Karhunen-Loeve expansion for simulation of stochastic processes, International journal for numerical methods in engineering 52 (9) (2001) 1029-1043.

[188] J. Hoffman, L. Anders, FEniCS/puffin user manual, Tech. rep., KTH Royal Institute of Technology (2013).

URL https: / / fenicsproject.org/pub/documents/puffin/

[189] P. G. Constantine, M. S. Eldred, E. T. Phipps, Sparse pseudospectral approximation method, Computer Methods in Applied Mechanics and Engineering 229 (2012) 1-12.

[190] R. Li, R. Ghanem, Adaptive polynomial chaos expansions applied to statistics of extremes in nonlinear random vibration, Probabilistic engineering mechanics 13 (2) (1998) $125-136$.

[191] D. Lucor, G. E. Karniadakis, Adaptive generalized polynomial chaos for nonlinear random oscillators, SIAM Journal on Scientific Computing 26 (2) (2004) 720-735.

[192] X. Wan, G. Karniadakis, An adaptive multi-element generalized polynomial chaos method for stochastic differential equations, Journal of Computational Physics 209 (2) (2005) 617-642.

[193] G. Lin, C.-H. Su, G. E. Karniadakis, Predicting shock dynamics in the presence of uncertainties, Journal of Computational Physics 217 (1) (2006) 260-276.

[194] X. Wan, G. E. Karniadakis, Beyond Wiener-Askey expansions: handling arbitrary pdfs, Journal of Scientific Computing 27 (1-3) (2006) 455-464.

[195] L. Liu, Y. Wong, B. Lee, Non-linear aeroelastic analysis using the point transformation method, part 1: Freeplay model, Journal of Sound and Vibration 253 (2) (2002) 447-469.

[196] X. Wan, G. Karniadakis, Long-term behavior of polynomial chaos in stochastic flow simulations, Computer methods in applied mechanics and engineering 195 (41) (2006) 5582-5596.

[197] O. Le Mattre, H. N. Najm, R. Ghanem, O. Knio, Multi-resolution analysis of Wienertype uncertainty propagation schemes, Journal of Computational Physics 197 (2) (2004) 502-531.

[198] D. R. Millman, P. I. King, R. C. Maple, P. S. Beran, L. K. Chilton, Uncertainty quantification with a B-spline stochastic projection, AIAA journal 44 (8) (2006) 1845-1853.

[199] M. Gerritsma, J.-B. Van der Steen, P. Vos, G. Karniadakis, Time-dependent generalized polynomial chaos, Journal of Computational Physics 229 (22) (2010) 8333-8363.

[200] T. Mathew, Domain decomposition methods for the numerical solution of partial differential equations, Vol. 61, Springer Science \& Business Media, 2008. 
[201] S. Badia, A. F. Martín, J. Principe, A highly scalable parallel implementation of balancing domain decomposition by constraints, SIAM Journal on Scientific Computing 36 (2) (2014) C190-C218.

[202] C. Geuzaine, F. Remacle, GMSH: A 3-D finite element mesh generator with built-in pre-and post-processing facilities, International Journal for Numerical Methods in Engineering 79 (11) (2009) 1309-1331.

[203] G. Karypis, V. Kumar, METIS-unstructured graph partitioning and sparse matrix ordering system, version 2.0 (1995).

[204] J. Ahrens, B. Geveci, C. Law, ParaView: An end-user tool for large-data visualization (2005).

[205] Graham-cedar: New national heterogeneous HPC clusters managed by Compute Canada (2017).

URL https://docs.computecanada.ca/wiki/National_systems

[206] Guillimin - HPC cluster managed by Calcul Québec and Compute Canada (2016).

URL http: / / www.hpc.mcgill.ca

[207] S. Badia, A. F. Martín, J. Principe, Multilevel balancing domain decomposition at extreme-scales, SIAM Journal on Scientific Computing 38 (1) (2016) C22-C52.

[208] B. F. Smith, An optimal domain decomposition preconditioner for the finite element solution of linear elasticity problems, SIAM Journal on Scientific and Statistical Computing 13 (1) (1992) 364-378.

[209] M. Dryja, O. B. Widlund, Some domain decomposition algorithms for elliptic problems, in: Iterative methods for large linear systems, Elsevier, 1990, pp. 273-291.

[210] J. Mandel, Two-level domain decomposition preconditioning for the p-version finite element method in three dimensions, International Journal for Numerical Methods in Engineering 29 (5) (1990) 1095-1108.

[211] J. Mandel, Iterative solvers by substructuring for the p-version finite element method, Computer Methods in Applied Mechanics and Engineering 80 (1-3) (1990) 117-128.

[212] Niagara supercomputer: New national homogeneous HPC clusters managed by Compute Canada (2018).

URL https: / / docs.computecanada.ca/wiki/Niagara

[213] C. Vuik, Krylov subspace solvers and preconditioners, ESAIM: Proceedings and Surveys 63 (2018) 1-43.

[214] A. Doostan, R. G. Ghanem, J. Red-Horse, Stochastic model reduction for chaos representations, Computer Methods in Applied Mechanics and Engineering 196 (37-40) (2007) 3951-3966. 
[215] R. G. Ghanem, A. Doostan, J. Red-Horse, A probabilistic construction of model validation, Computer Methods in Applied Mechanics and Engineering 197 (29-32) (2008) 2585-2595.

[216] A. Klawonn, O. B. Widlund, Dual-primal FETI methods for linear elasticity, Communications on Pure and Applied Mathematics: A Journal Issued by the Courant Institute of Mathematical Sciences 59 (11) (2006) 1523-1572.

[217] A. Klawonn, O. Rheinbach, A parallel implementation of dual-primal FETI methods for three-dimensional linear elasticity using a transformation of basis, SIAM Journal on Scientific Computing 28 (5) (2006) 1886-1906.

[218] A. Klawonn, O. Rheinbach, Inexact FETI-DP methods, International Journal for Numerical Methods in Engineering 69 (2) (2007) 284-307.

[219] J. Li, O. B. Widlund, On the use of inexact subdomain solvers for BDDC algorithms, Computer Methods in Applied Mechanics and Engineering 196 (8) (2007) 1415-1428.

[220] H. C. Elman, V. Forstall, Preconditioning techniques for reduced basis methods for parameterized elliptic partial differential equations, SIAM Journal on Scientific Computing 37 (5) (2015) S177-S194.

[221] N. Chrisochoides, Multithreaded model for the dynamic load-balancing of parallel adaptive PDE computations, Applied Numerical Mathematics 20 (4) (1996) 349-365.

[222] Gaurav, S. F. Wojtkiewicz, Use of GPU computing for uncertainty quantification in computational mechanics: A case study, Scientific Programming 19 (4) (2011) 199-212.

[223] A. A. Contreras, P. Mycek, O. Le Ma1tre, F. Rizzi, B. Debusschere, O. M. Knio, Parallel domain decomposition strategies for stochastic elliptic equations-part a: Local kl representations.

[224] A. Alexanderian, A brief note on the karhunen-lo $\backslash$ eve expansion, arXiv preprint arXiv:1509.07526.

[225] D. Xiu, G. Karniadakis, Modeling uncertainty in flow simulations via generalized polynomial chaos, Journal of Computational Physics 187 (1) (2003) 137-167.

[226] M. Stein, Large sample properties of simulations using latin hypercube sampling, Technometrics 29 (2) (1987) 143-151.

[227] W. L. Oberkampf, M. F. Barone, Measures of agreement between computation and experiment: validation metrics, Journal of Computational Physics 217 (1) (2006) 5-36. 